ID 6387

ERNEST $\square R L A N D G$ LAWRENEE

BERKELEY NATIDNAL LABGRATロRY

\title{
Two-Dimensional Nuclear \\ Magnetic Resonance of \\ Quadrupolar Systems
}

Shuanhu Wang

Materials Sciences Division

RECEIVEO

MAR 181999

OSTI

September 1997

Ph.D. Thesis 


\section{DISCLAIMER}

This document was prepared as an account of work sponsored by the United States Government. While this document is believed to contain correct information, neither the United States Government nor any agency thereof, nor The Regents of the University of California, nor any of their employees, makes any warranty, express or implied, or assumes any legal responsibility for the accuracy, completeness, or usefulness of any information, apparatus, product, or process disclosed, or represents that its use would not infringe privately owned rights. Reference herein to any specific commercial product, process, or service by its trade name, trademark, manufacturer, or otherwise, does not necessarily constitute or imply its endorsement, recommendation, or favoring by the United States Government or any agency thereof, or The Regents of the University of California. The views and opinions of authors expressed herein do not necessarily state or reflect those of the United States Government or any agency thereof, or The Regents of the University of California.

Ernest Orlando Lawrence Berkeley National Laboratory is an equal opportunity employer. 


\section{DISCLAIMER}

Portions of this document may be illegible in electronic image products. Images are produced from the best available original document. 


\title{
Two-Dimensional Nuclear Magnetic Resonance of Quadrupolar Systems
}

\author{
Shuanhu Wang \\ Ph.D. Thesis \\ Department of Chemistry \\ University of California, Berkeley \\ and \\ Materials Sciences Division \\ Ernest Orlando Lawrence Berkeley National Laboratory \\ University of California \\ Berkeley, CA 94720
}

September 1997

This work was supported by the Director, Office of Energy Research, Office of Basic Energy Sciences, Materials Sciences Division, of the U.S. Department of Energy under Contract No. DE-AC03-76SF00098. 
Two-Dimensional Nuclear Magnetic Resonance of Quadrupolar Systems

\section{Copyright (C) 1997}

\section{by}

Shuanhu Wang

The U.S. Department of Energy has the right to use this thesis for any purpose whatsoever including the right to reproduce all or any part thereof 
Two-Dimensional Nuclear Magnetic Resonance of Quadrupolar Systems

by

\author{
Shuanhu Wang \\ Doctor of Philosophy in Chemistry \\ University of California at Berkeley \\ Professor Alexander Pines, Chair
}

\begin{abstract}
This dissertation describes two-dimensional nuclear magnetic resonance theory and experiments which have been developed to study quadrupoles in the solid state. The technique of multiple-quantum magic-angle spinning (MQMAS) is extensively reviewed and expanded upon in this thesis. Specifically, MQMAS is first compared with another technique, dynamic-angle spinning (DAS). The similarity between the two techniques allows us to extend much of the DAS work to the MQMAS case. Application of MQMAS to a series of aluminum containing materials is then presented. The superior resolution enhancement through MQMAS is exploited to detect the five- and six-coordinated aluminum in many aluminosilicate glasses. Combining the MQMAS method with other experiments, such as HETCOR, greatly expands the possibility of the use of MQMAS to study a large range of problems and is demonstrated in Chapter 5. Finally, the technique switching-angle spinning (SAS) is applied to quadrupolar nuclei to fully characterize a quadrupolar spin system in which all of the 8 NMR parameters are accurately determined. This dissertation is meant to demonstrate that with the combination of two-dimensional NMR concepts and new advanced spinning technologies, a series of multiple-dimensional NMR techniques can be designed to allow a detailed study of quadrupolar nuclei in the solid state.
\end{abstract}




\section{Contents}

List of Tables vii

List of Figures $\quad$ ix

Acknowledgements xiii

1 Introduction 1

2 Basic NMR Theory 3

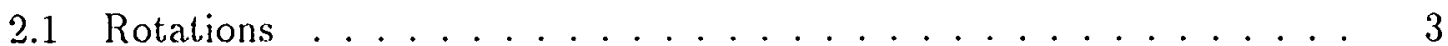

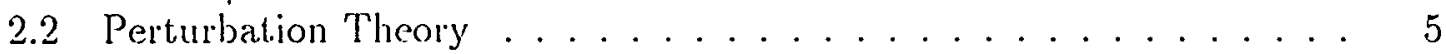

2.3 Nuclear Spin Hamiltonians . . . . . . . . . . . . . 6

2.3.1 Zeeman Interaction ............... 7

2.3.2 Quadrupolar Interaction .............. 8

2.3.3 Chemical Shift Interaction . . . . . . . . . 11

2.3.4 Dipolar Interaction ................ 13

2.4 Perturbative Treatment of Spin Hamiltonians . . . . . . . . 14

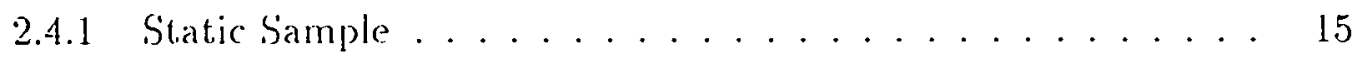

2.4 .2 Spinning Samples ................... 16

2.5 Evolution of Spin Systom . . . . . . . . . . . . 23

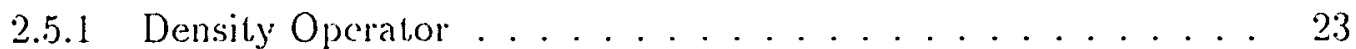

2.5.2 Evolution Hnder RF pulses ............. 27

2.5 .3 Free Induction Drcay . . . . . . . . . . . . 30

2.5.4 Example: A Simple ID NMIR Experiment ........ 32

2.5.5 Coherence Pahway ................. 33 
2.6 Two-Dimensional NMR . . . . . . . . . . . . 36

2.6 .1 Basics . . . . . . . . . . . . . 36

2.6.2 Pure-Absorption Phase 2-D NMR Lineshape . . . . . . . 37

3 High-Resolution NMR of Quadrupoles 40

3.1 Magic-Angle and Variable-Angle Spinning . . . . . . . . 40

3.2 2nd-order Averaging: Theory . . . . . . . . . . . . 44

3.2.1 Hahn-Echo Experiment. . . . . . . . . . . . . . 44

$3.2 .2 \quad$ DAS $\ldots \ldots \ldots \ldots \ldots \ldots \ldots \ldots$

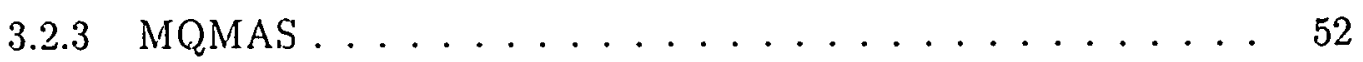

3.3 Phase Cycle . . . . . . . . . . . . . . . 58

3.4 Excitation and Reconversion Pulses . . . . . . . . . . 65

3.4.1 Fictitious Spin- $\frac{1}{2}$ Operators $\ldots \ldots \ldots \ldots 66$

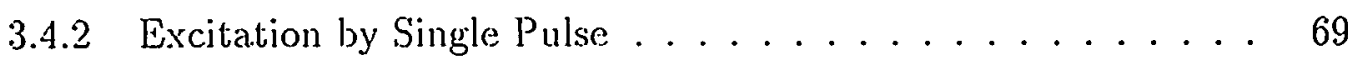

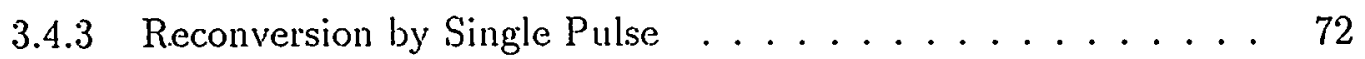

3.4.4 Excitation and Reconversion: Computer Simulation . . . . . 75

3.4.5 Excitation and Reconversion by Spin-Locking . . . . . 77

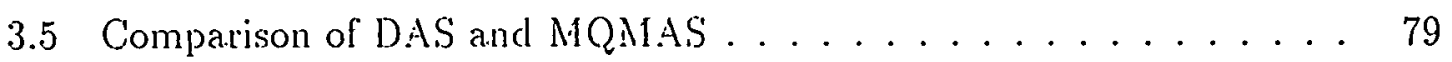

3.5 .1 Experiments. . . . . . . . . . . . . . 79

3.5 .2 Feasibility . . . . . . . . . . . . . 80

3.5 .3 Linewidth . . . . . . . . . . . . . 83

3.5.4 Resolution . . . . . . . . . . . . . . 85

3.5.5 Chemical Shift Effect. . . . . . . . . . . . . 87

3.5.6 Chemical Shift Anisotropy . . . . . . . . . . . . . 89

3.6 Multiple-Quantum Variable-Angle Spinning . . . . . . . . 93 


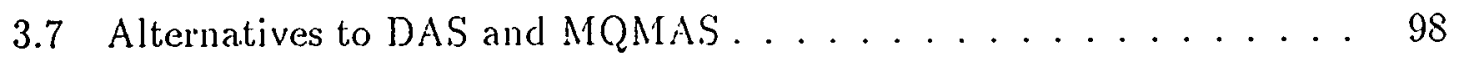

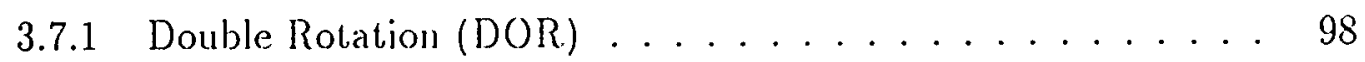

3.7 .2 Dynamic-Angle Hopping . . . . . . . . . . 99

4 Application of MQMAS to Aluminum-Containing Materials 101

4.1 Interpretation of MQMAS Spectra . . . . . . . . . . 102

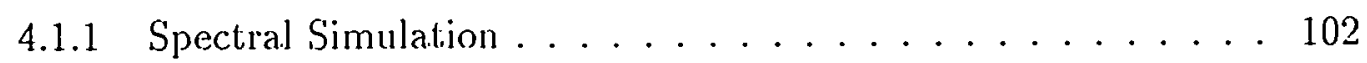

4.1.2 DAS and MQMAS: Extraction of $\delta_{i s o}$ and $P_{Q} \ldots 103$

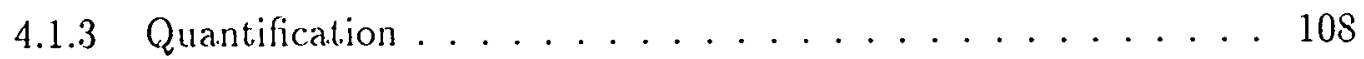

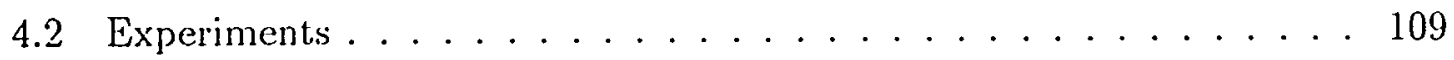

4.2 .1 Sample Preparation . . . . . . . . . . . 109

4.2 .2 NMR Spectroscopr .................... 109

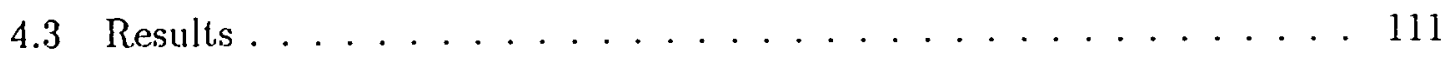

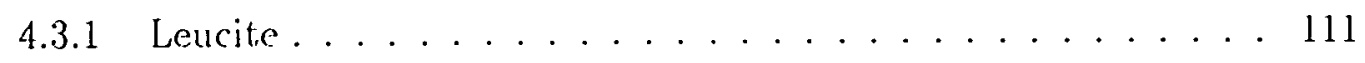

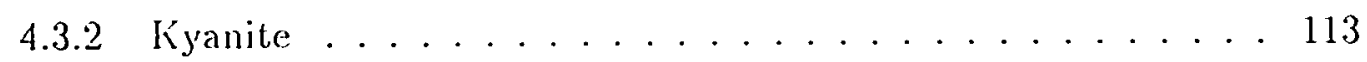

4.3.3 Crystalline Anorthite . . . . . . . . . . 113

4.3.4 Anorthite $\left(\mathrm{CaAl}_{2} \mathrm{Si}_{2} \mathrm{O}_{8}\right)$ Glass .............. 119

4.3.5 Magnesium Aluminoborat. Glass ............ 120

4.3.6 Goosecreckit. .................... 122

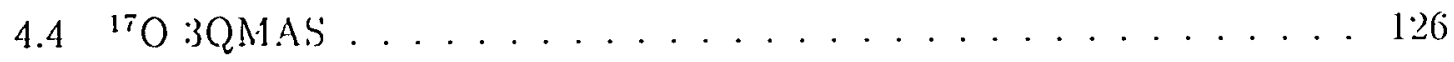

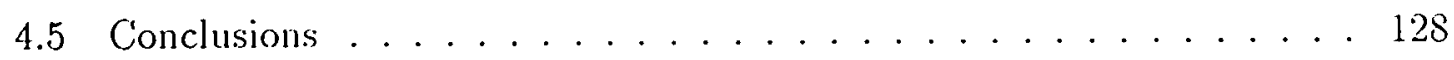

5 Correlation Spectroscopy with MQMAS 130

5.1 Heteronuclear Correlation (IIETCOR) ............ 130

5.2 HETCOR will Quadrupoles . . . . . . . . . . . . 134

5.3 High-Resolution IHET(') . . . . . . . . . . . 136 
5.4 Conclusion . . . . . . . . . . . . . . . 143

6 Switching-Angle Spinning of Quadrupoles $\quad 144$

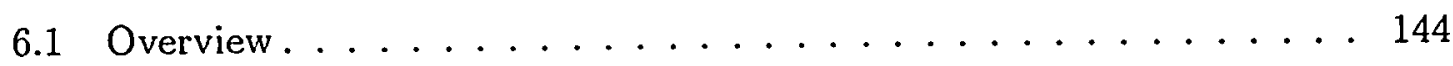

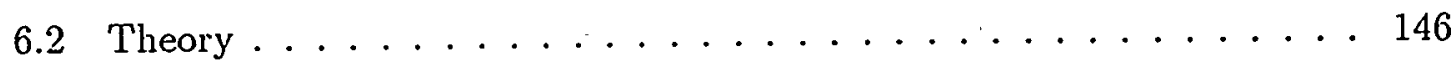

6.2 .1 Coexisting Tensors ........................ 146

6.2 .2 Switching-Angle Spinning ................ 148

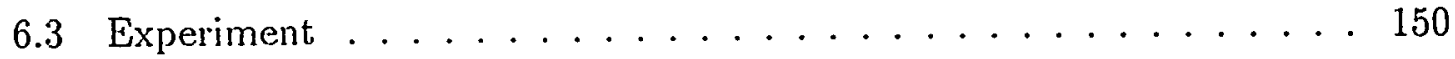

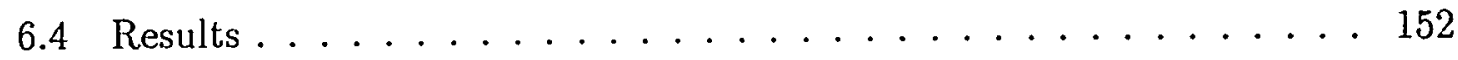

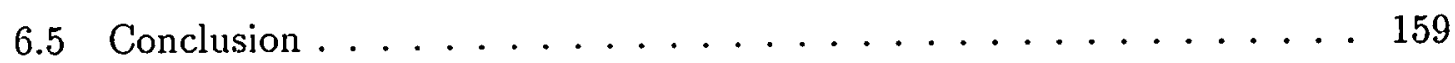

A A Short Review on MQMAS 163

$\begin{array}{ll}\text { Bibliography } & 174\end{array}$ 


\section{List of Tables}

2.1 Reduced Wigner rotation matrix elements $d_{n m}^{(2)}(\beta) \ldots \ldots \ldots$

2.2 Coefficients in the anisotropic cosine expansion for second-order quad-

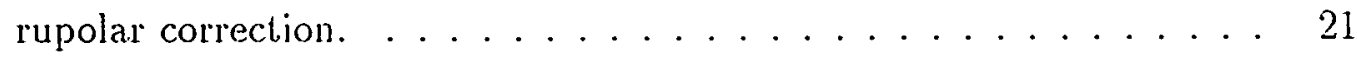

2.3 Coefficients of scaling factors $C_{0}, C_{1}$ and $C_{2} \ldots \ldots \ldots 22$

3.1 Coherence pathway and different scaling values for different spins. . 58

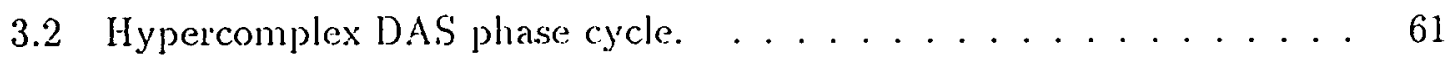

3.3 Hypercomplex MQMAS phase cycle. . . . . . . . . . 62

3.4 Hypercomplex SEDAS phase cycle. . . . . . . . . 63

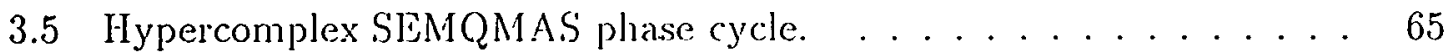

3.6 Pulse lengths that maximize the excitation and reconversion efficiency. 77

3.7 The spin-locking $3 Q \mathrm{QMSS}$ phase cycle. $\ldots \ldots \ldots \ldots \ldots$

3.8 Comparison of the DAS, MQMAS, echo and MAS linewidths for some model compounds. . . . . . . . . . . . . . . . 84

4.1 Isotropic shifts and quadrupolar coupling parameters for leucite. . . 105

4.2 Isotropic shifts and quadrupolar coupling parameters for crystalline anorthile $\left(11 . \tau^{\top} T\right) \ldots \ldots \ldots \ldots \ldots \ldots$

4.3 Isotropic slift.s and quadrupolar coupling paramoters for crystalline

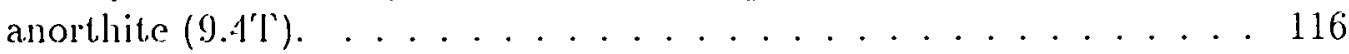

4.4 Comparison of Arnorthite NMR parameters from single-crystal and powdered sample studies. . . . . . . . . . . . . . . 118

4.5 Isotropic chemical shifts and quadrupolar coupling products derived for goosecreckitc. . . . . . . . . . . . . . . . . . . . . 124 
5.1 Phase cycles for MQMAS/HETCOR . . . . . . . . 139 


\section{List of Figures}

2.1 The Euler angles between two reference frames. . . . . . . . . 4

2.2 Zeeman, first- and second-order energy splittings for $I=\frac{3}{2}$ nucleus. . $\quad 17$

2.3 PAS to rotor to lab frame Transformations. . . . . . . . . 18

2.4 Treatment of a time-dependent Hamiltonian. $\ldots \ldots \ldots \ldots \ldots$

2.5 Pulse sequence for a simple one-dimensional NMR experiment. . . . 32

2.6 Coherence pathway for a simple DAS experiment. . . . . . . 34

2.7 A schematic diagram of two-dimensional NMR. . . . . . . . . 37

3.1 Simulated static and MAS spectra of a spin- $\frac{1}{2}$ nucleus. . . . . . 41

3.2 2nd and 4th order Legendre polynomials as a function of spinner angle. 43

$3.3{ }^{23} \mathrm{Na}$ static and MAS spectra of $\mathrm{Na}_{2} \mathrm{C}_{2} \mathrm{O}_{4}$ at $9.4 \mathrm{~T} . \quad \ldots \ldots \ldots . \ldots 43$

3.4 Simulated guadrupolar variable-angle spinning (VAS) spectra. $\ldots . \quad 45$

$3.590^{\circ}-180^{\circ}$ Hahn-echo secquence and coherence pathway. . . . . . 46

3.6 Hahn-echo spectrum of ${ }^{s 7} \mathrm{Rb}$ in $\mathrm{RbNO}_{3}$ at $11.7 \mathrm{~T} . \ldots \ldots 47$

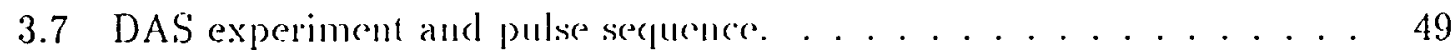

3.8 DAS angle pairs as a function of $k \ldots \ldots \ldots \ldots \ldots \ldots$

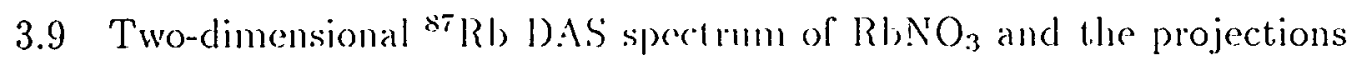
in both dimensions. . . . . . . . . . . . . . . . . . 53

3.10 MQMAS experiment, pulse secuence and coherence pathway. . . . 54

3.11 Two-dimensional ${ }^{87} \mathrm{Rb} 3 \mathrm{BQMAS}$ spectrum of $\mathrm{RbNO}_{3}$ and the projections in both dimansions. . . . . . . . . . . . . . . 57 
3.12 Hypercomplex DAS and MQMAS experiment. . . . . . . . 60

3.13 HyperSEDAS and HyperSEMQMAS experiments. $\ldots \ldots \ldots \ldots 64$

3.14 Calculated triple-quantum excitation efficiency for single crystal and powder samples. . . . . . . . . . . . . . . . . 73

3.15 Experimental data on triple-quantum excitation and reconversion efficiency. . . . . . . . . . . . . . . . . . 74

3.16 Calculated triple-quantum lo single-quantum reconversion efficiency. $\quad 76$

3.17 Spin-Locking pulse sequence for the excitation of triple-quantum coherence and its conversion back to single-quantum coherence. . . . 78

3.18 3QMAS spectra of many nuclei. . . . . . . . . . . 82

3.19 9.4T DAS and MQMAS spectra of ${ }^{17} \mathrm{O}$ labeled stilbite. . . . . . 86

3.20 Comparison of the resonance frequencies of DAS, 3QMAS and 5QMAS for amorphous $\mathrm{Si}^{17} \mathrm{O}_{2}$ and coesite. $\ldots \ldots \ldots \ldots \ldots$

$3.2111 .7 \mathrm{~T}$ MQMAS spect.rum of ${ }^{87} \mathrm{Rb}$ in $\mathrm{Rb}_{2} \mathrm{CrO}_{4} \ldots \ldots \ldots$. . . . . 91

3.22 Sumulated 11.7T MASs spectirum of ${ }^{87} \mathrm{Rb}$ in $\mathrm{Rb}_{2} \mathrm{CrO}_{4}$. . . . . . 92

3.23 11.7T DAS, MQNAS and simulated MQMAS spectra for ${ }^{87} \mathrm{Rb}$ in $\mathrm{Rb}_{2} \mathrm{CrO}_{4} \ldots \ldots \ldots \ldots \ldots \ldots \ldots$

$3.2411 .7 \mathrm{~T}$ 3QVAS spectrum of ${ }^{8} \mathrm{Rb}$ in $\mathrm{RbNO}_{3} . \ldots \ldots \ldots \ldots \ldots$

3.25 Simulated two-dimensional 3QV AS patterns with different Euler angles. 98

$4.1{ }^{27} \mathrm{Al}$ 3QNAS NMR spectrum for leucite. . . . . . . . . 106

4.2 Least-square fit of 11.7T and 9.4T MAS and 3QMAS shifts for leucite. 107

$4.3{ }^{27} \mathrm{Al}$ MAS spect.ra of Lcucite. . . . . . . . . . . . . . . . 112

$4.4{ }^{2 \pi} \mathrm{Al}$ MAS and 3QMAS spectra for kyanite. . . . . . . . . 114 
$4.5{ }^{27} \mathrm{Al}$ 3QMAS NMR spect.rum for crystal anorthite at 11.7T. . . . 115

4.6 MAS Projection from 11.7T :3QMAS spectrum of anorthite. . . . . 117

4.7 Isotropic chemical shifts for anorthite against the mean $\mathrm{Si}-\mathrm{O}-\mathrm{Al}$ angle

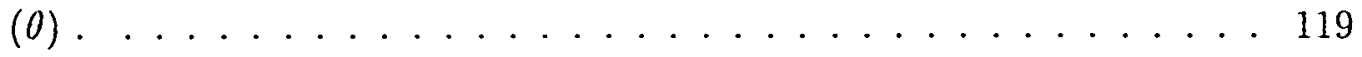

4.8 ${ }^{27} \mathrm{Al} 3 \mathrm{QMAS}$ NMR spect.rum for $\mathrm{CaAl}_{2} \mathrm{Si}_{2} \mathrm{O}_{8}$ glass at $11.7 \mathrm{~T}$. . . . 121

$4.9{ }^{27} \mathrm{Al}$ 3QMAS NMIR spectrum for a glass of magnesium borate glass. $\quad 123$

4.1011 .7 T 3QMAS and 5QMAS spectra of goosecreekite. . . . . . . 125

$4.1111 .7 \mathrm{~T}^{17} \mathrm{O}$ 3QMAS of larnite and forsterite. . . . . . . . 127

5.1 Cross-Polarization between two spins I and S. . . . . . . . 131

5.2 Pulse sequence and colerence pathway for a traditional HETCOR experiment. . . . . . . . . . . . . . . 134

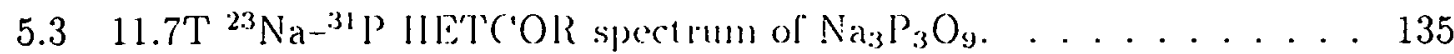

5.4 Comparison of DAS/IIE'TCOOR and MQMAS/HETCOR pulse sequences and coherence transfer pathways. ..........................

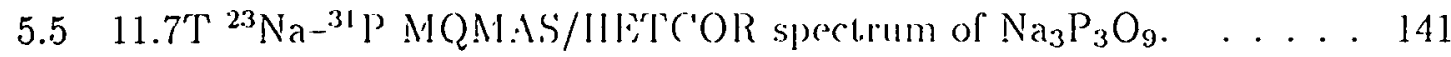

6.1 Relationship bewwern quadrupolar and clemical shift principle axis

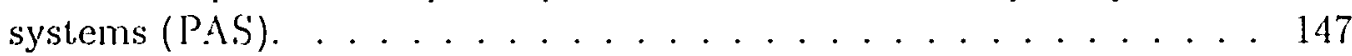

6.2 Switching-angle spinning $(S . S S)$ experincnt. . . . . . . . . . 149

6.3 Simulated two-dimensional SilS spectra. ............ 151

6.4 4.2T experimental and simulated ${ }^{23} \mathrm{Na}$ S.AS specta of $\mathrm{Na}_{2} \mathrm{SO}_{4} . \quad \ldots \quad 152$

6.59.4'T experimental and simmlated ${ }^{\mathrm{N}} \mathrm{Rb}$ S.AS NMIR spectara of $\mathrm{R}_{2} \mathrm{SO}_{4}$. 154

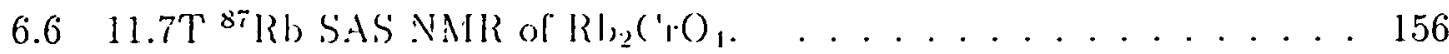


6.7 Experimental and simulated two-dimensional ${ }^{87}$ R.b SAS NMR spectra

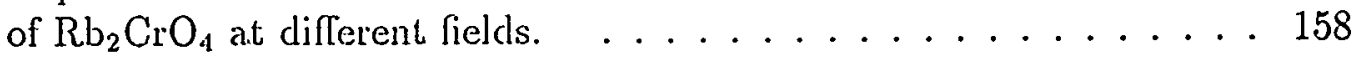

6.8 Dependence of ${ }^{87} \mathrm{Rb}$ SAS NMR spectra of $\mathrm{Rb}_{2} \mathrm{CrO}_{4}$ on chemical shift parameters. . . . . . . . . . . . . . . 160

6.9 Dependence of ${ }^{87} \mathrm{Rb} S A S$ NMR spectra of $\mathrm{Rb}_{2} \mathrm{CrO}_{4}$ on Euler angles between the CSA and QI PAS. . . . . . . . . . . 161 


\section{Acknowledgements}

A Chinese proverb says that three of the most enjoyable things in life are: To achieve a carecr goal successfully, to marry somebody that you love, and to meet old friends when you are far away from home. This is a very good summary of my graduate life at Berkeley.

Getting a Ph.D. at Berkeley was not, even in my wildest dream. Born in a small village in China, my carecr could simply end after high school. Without the encouragements and supports from my parents. sisters, brothers and my teachers in high school, I would not have been to a top university in China, let alone to come to Berkeley later. I would like to thank Professor Alexander Pines, who has created a very active researching cuviromment that at, tracts so many smart people around the world to the group. Working with these prople is the greatest thing one can imagine. I am greatly indebted to Dr. Jay Shore. who led a new graduate student into the field of NMR, and spent so much lime and put so much effort to help me out whenever I had trouble with the experincents. Professor Alexis Boll was another great mentor that taught me how to decompose, analyzo and atlack each hard problem in my first year at Berkeley. I would like also to lhank 1)r. Jeff Yarger, who is not only a great collaborator, but also a compulcr lover whom I can share my computer experience with. While it is hard to list all of the people that have been so helpful to me, I want to give my heartful haanks 10 1)r. Russell Larsen, Andrew Liolbert, Yungya Lin, Susan De Paul, and Mallliass Ermst.

I should not forget my collaborators at Stanford and Santa Barbara. Without Dr. Zhi Xu and Prof. Jonathan Stobbins, who constantly provide me interesting samples, I could not have finished my work now. Dr. Lucy Bull and Susan De Paul are my collaborators on the MQMAS/IIETCOR project. Without their experience 
on cross-polarization and probe design, the project would last much longer. Dr. Jay Baltisberger deserves my special thanks for his help in setting up many of the experiments.

It is during my graduate school time that; I met my wife and got married. Being a Berkeley man has made my life much easier. I do not need to take special efforts to convince my wife to marry me; Instead, I only need to let her know my shortcomings.

My life at Berkeley would have been really tough if not for the help of all my good friends here. It is not possible for me to use more space than just list some of their names: Zhan Chen, Jiulong Meng, Weiling Wang, Bin He, Yulin Lou, Wenzhong Xiao, Qian Fu, Suli Fei, Meihong, Baohua Huang, Xiaodong Sun. 


\section{Chapter 1}

\section{Introduction}

More than $60 \%$ of the isotopes in the periodic table have an uneven distribution of nuclear charges, and thus an electric quadrupole moment. The coupling between the nuclear quadrupole moment and the electric field gradient (EFG) generated at the center of the nucleus gives an electric quadrupolar interaction that dominates all other internal spin interactions (such as chemical shift, and dipolar interactions) that a quadrupolar nucleus experiences. The nuclear magnetic resonance (NMR) spectra of quadrupoles are often broadened by this interaction, whose second-order effect can not be efliciently removed by the conventional sample rotation method magicangle spinning (MAS). The low resolution of the resultant spectra greatly limits the applicability of NMR to various important materials, and requires novel techniques to give liquid-like resolution in the solid-state.

Dynamic-angle spinning (D.AS) $[1,2]$ and multiple-guantum magic-angle spinning (MQMAS) [3] are two of the most important developments that overcome the resolution problem. DAS, first realized at Berkeley in 1990). has evolved into a relatively mature technique by now; the MIQMASS mothod proposed by L. Frydman in 1995, however, is showing greal promise and has some intrinsic advantages over DAS. This thesis describes the MOQNAS and DSLS experiments I performed at Berkeley that represent some newer contributions to high-resolution NMR of guadrupoles. Even though my research has bern focusing on .ICQNAS. Ho great similarity between DAS and MQMAS makes it desirable 1.o present both techniques in parallel, and compare them when possible.

Following this short introduction. chapher 2 prescents some basic NMR theories 
that are used throughout this thesis. In chapter 3, DAS and MQMAS are discussed and compared to each other in great detail. The similarities between the two techniques are clearly demonstrated and various developments based on DAS are shown to be equally applicable to MQMAS. Chapter 4 applies MQMAS to ${ }^{27} \mathrm{Al}$-containing minerals and zeolites. The advantages of MQMAS over MAS is illustrated in both crystalline and glass samples. Preliminary application of MQMAS to ${ }^{17} \mathrm{O}$ is also given. Chapter 5 shows another interesting experiment that utilizes MQMAS to give high-resolution HETCOR spectira for quadrupoles.

The last chapter in this thesis focuses on the switching-angle spinning (SAS) technique that allows the determination of chemical shift parameters along with the relative orientation between the chemical shift and quadrupolar principal axis systems (PAS). Compared to conventional one-dimensional techniques, this method is more accurate and reliable, when the magnitude of chemical shift interaction is moderate.

Since MQMAS is now a hot topic in the NMR. community, great advances have been achieved in the past lwo years. A brief review of this area is given in the appendix. With this revicw, the thesis becomes a complete reference for highresolution NMR of quadrupoles. 


\section{Chapter 2}

\section{Basic NMR Theory}

The wealth of structural and dynamic information in NMR experiments comes from the combination of various nuclear spin interactions and the external perturbations applied to the system. The nuclear spin intcractions affect the eigenstates of the system, therefore change the experimental NMR spectra. The external perturbations generated by RF fields can be manipulated with arbitrary flexibility to selectively utilize or average out sprecific intcraction(s), leaving us with spectra manifesting different aspects of the system. There have been many good books that provide general discussions of thic principles of nuclear magnetic resonance $[4,5,6,7]$ and its application to solid state materials $[\delta, 9]$. In the current chapter, I will only include some funclamentals of NMR theory that are used in the later chapters to describe the more advanced NMR experiments.

\section{$2.1 \quad$ Rotations}

All techniques used for selective averaging in NMR rely on rotations of one or another kind of the intermal Hantilonians. The belavior of any physical property under rotation can be studied more casily when llwe propery is expressed in terms of irreducible tensor operators [10]. The reason is that the transformation of the tensors under rotation is woll kinown.

Rotations in NMR take two distinct forms: rotalions in spin space and rotations in real space. Each NMR IIamiltonian can be written as a summation of product of 
spin and spatial tensors as shown below.

$$
H_{\lambda}=\sum_{l} \sum_{n=-l}^{l}(-1)^{m} R_{l,-m}^{\lambda} T_{l m}^{\lambda}
$$

The tensor components $R_{l m}^{l}$ and $T_{l m}^{\lambda}$ are the spatial and spin tensor components, respectively. The rotations in each space only affect the variables in that space, thus by writing the Hamiltonians in the above form, the spin and real spaces are decoupled and handled separately.

A rotation is often specified by three Euler angles $(\alpha, \beta, \gamma)$ between the two reference frames before and after rotation, as shown in Figure 2.1. An lth-rank tensor then transforms in the following way.

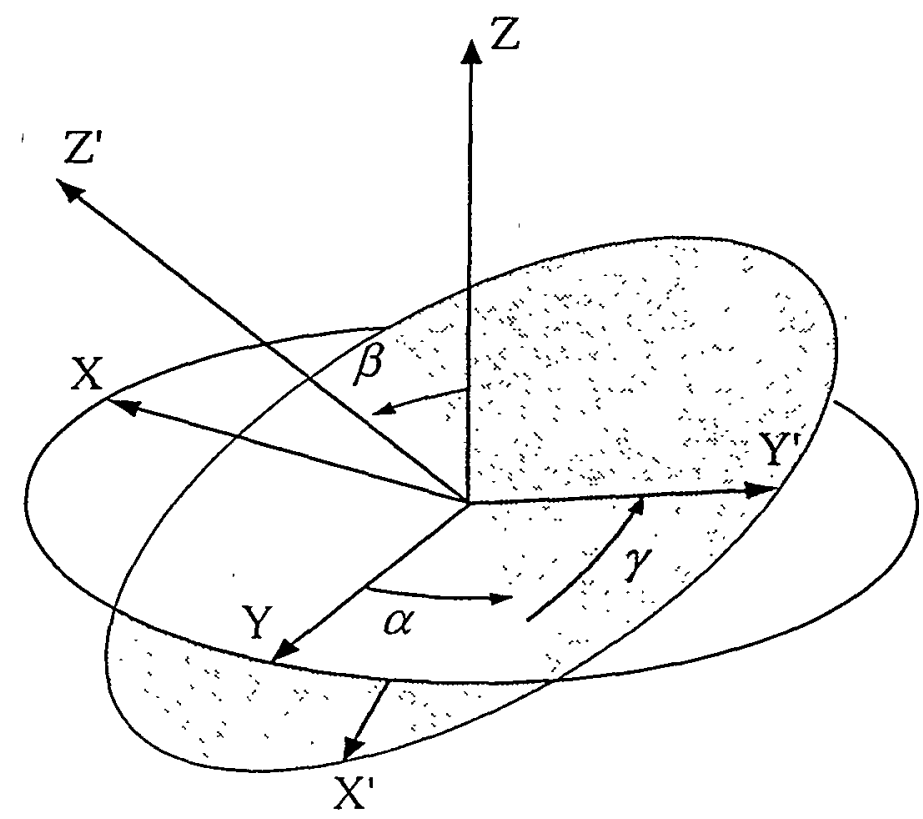

Figure 2.1: The Euler angles $a, \beta, \gamma$ that describe the transformation between two reference frames.

$$
A_{m}^{\prime}=\sum_{n=-l}^{l} D_{n m}^{(l)}(\alpha, \beta, \gamma) \cdot A_{l n}
$$

Here, $A$ and $A^{\prime}$ arc tensors in the original and new frames. The evaluation of tensor 
$D^{(l)}$ is facilitated by the reduced Wigner rolation matrix clements $d_{n m}^{(l)}$.

$$
D_{n m}^{(l)}(\alpha, \beta, \gamma)=e^{-i(m \alpha+n \gamma)} d_{n m}^{(l)}(\beta)
$$

For most of the work in this thesis, I will deal primarily with second-order tensors in the spin and spatial spaces. Therefore, a table of sccond-order Wigner matrices is included here for further references.

\begin{tabular}{|c||c|c|c|c|c|}
\hline$n$ & 2 & 1 & $m$ & 0 & -1 \\
\hline \hline 2 & $\left(\frac{1+\cos \beta}{2}\right)^{2}$ & $-\frac{1+\cos \beta}{2} \sin \beta$ & $\sqrt{\frac{3}{8}} \sin ^{2} \beta$ & $-\frac{1-\cos \beta}{2} \sin \beta$ & -2 \\
\hline 1 & $\frac{1+\cos \beta}{2} \sin \beta$ & $\cos ^{2} \beta-\frac{1-\cos \beta}{2}$ & $-\sqrt{\frac{3}{8}} \sin 2 \beta$ & $\frac{1+\cos \beta}{2}-\cos ^{2} \beta$ & $-\frac{1-\cos \beta}{2} \sin \beta$ \\
\hline 0 & $\sqrt{\frac{3}{8}} \sin ^{2} \beta$ & $\sqrt{\frac{3}{8}} \sin 2 \beta$ & $\frac{3 \cos ^{2} \beta-1}{2}$ & $-\sqrt{\frac{3}{8}} \sin 2 \beta$ & $\sqrt{\frac{3}{8}} \sin ^{2} \beta$ \\
\hline-1 & $\frac{1-\cos \beta}{2} \sin \beta$ & $-\cos ^{2} \beta+\frac{1+\cos \beta}{2}$ & $\sqrt{\frac{3}{8}} \sin ^{2} \beta$ & $-\frac{1-\cos \beta}{2}+\cos ^{2} \beta$ & $-\frac{1+\cos \beta}{2} \sin \beta$ \\
\hline-2 & $\left(\frac{1-\cos \beta}{2}\right)^{2}$ & $\frac{1-\cos \beta}{2} \sin \beta$ & $\sqrt{\frac{3}{8}} \sin ^{2} \beta$ & $\frac{1+\cos \beta}{2} \sin \beta$ & $\left(\frac{1+\cos \beta}{2}\right)^{2}$ \\
\hline
\end{tabular}

Table 2.1: Reduced Wigner rotation matrix elements $d_{n m}^{(2)}(\beta)$

\subsection{Perturbation Theory}

Static perturbation theory [11, 12] is used throughout this thesis to analyze the effects of the nuclear spin Ilamiltonians on the resultant NMIR spectra. The theory is useful when the Ilamiltonian can bo writuen as

$$
I I=I_{0}+\lambda I^{\circ}
$$

where $V \ll I_{0}$ and $0 \leq \lambda \leq 1$. Assmming l hat I lo cigenstates and eigenvalues of $H_{0}$ are $\mid n>$ and $\varepsilon_{n}$, that is

$$
I_{0}\left|n>=\varepsilon_{n}\right| n>
$$

perturbation theory makes the assumption that the cigenstates and eigenvalues of $H$ are obtained by adding higher order comedion terms to those of the unperturbed 
system.

$$
\begin{aligned}
\mid N> & =|n>+\lambda| N^{(1)}>+\lambda^{2} \mid N^{(2)}>+\cdots \\
\varepsilon_{N} & =\varepsilon_{n}+\lambda \varepsilon_{N}^{(1)}+\lambda^{2} \varepsilon_{N}^{(2)}+\cdots
\end{aligned}
$$

Here, $\left|N^{(i)}\right\rangle$ and $\varepsilon_{N}^{(i)}$ are a. series of correction terms to the eigenstate $|n\rangle$ and eigenvalue $\varepsilon_{n}$. Inserting Egns 2.6 and 2.7 into

$$
|H| N^{i}>=\left(H_{0}+\lambda V\right)\left|N>=\varepsilon_{N}\right| N>
$$

and collecting terms on both side of the equation with the same power of $\lambda$, the firstand second-order energy corrections can be obtained.

$$
\begin{gathered}
E_{N}^{(1)}=<n|V| n> \\
E_{N^{\prime}}^{(2)}=\sum_{n \neq m} \frac{<n|V| m><m|V| n>}{\varepsilon_{n}-\varepsilon_{m}}
\end{gathered}
$$

The result on the first-order energy correction states that, we need merely to calculate eigenvalue corrections using the original basis set. This is equivalent to ignoring all the terms in $l i$ that clo not commute with the basis Hamiltonian $H_{0}$ (nonsecular terms). Using a malrix representation, this means that all the non-diagonal matrix elements in $V$ are neglected and the Hamiltonian $H_{0}+V$ is approximated by a diagonal Hamiltonian. This process of truncating Hamiltonians has proved essential in the treatment of internal spin Hamiltonians in NMR.

\subsection{Nuclear Spin Hamiltonians}

The nuclear spin Hamiltonians consist of a. number of terms that describe physically different interactions of the nuclear spins.

$$
I I=I_{Z}+H_{H H}+H_{Q}+H_{C S}+H_{D}
$$


The first two terms, $H_{\%}$ and $H_{H} \%$, reflect the coupling of the nuclear magnetic dipole moment with the external static and RF magnolic ficlds. These fields are controlled by the experimenter. 'The last three terms are determined by the local environment around the nucleus instead. Of all the spin interactions mentioned here, only the electric quadrupolar interaction will be discussed in any details, as the thesis is mainly concerned with half-intorger quadrupolar nuclej.

\subsubsection{Zeeman Interaction}

The single strongest, interaction in high-field NMR experiment is the Zeeman interaction, whose magnitude is proportional to the static magnetic field strength $B_{0}$. This direction of $B_{0}$ also defines the z-axis of the laboratory frame.

$$
I_{z}=-\operatorname{li\gamma } B_{0} I_{0}=-\operatorname{lic} \omega_{0} I_{0}
$$

In this equation, $\gamma$ is a ratio between the nuclear dipole moment and the nuclear angular momentum (gyromagnotic ratio), and $\omega_{0}$ is the Larmor frequency. The spin operator

$$
l_{0}=I_{Z}
$$

is one of the three spherical opcrators making up the complete spin operator set. The other two are defined below.

$$
l_{ \pm}= \pm \frac{1}{\sqrt{2}}\left(I_{x} \pm i l_{1}\right)
$$

The eigenstales of the dominating /erman Hamiltonian form a basis set for the perturbative lratmont of ofluer mucloar spin interactions. A nuclear spin with quantum numbel $I$ will have $2 I+1$ cigenstales, ach denoted as $\mid I m>$ where $-I \leq m \leq l$. In he hasis set formed by lhese eigenstates, the matrix elements 
of the spin operatiors are given below.

$$
\begin{aligned}
& I_{0} \mid I m>=m \\
& I_{ \pm}\left|I m>= \pm \frac{1}{\sqrt{2}} \sqrt{(I \mp m)(I \pm m+1)}\right| I, m \pm 1>
\end{aligned}
$$

\subsubsection{Quadrupolar Interaction}

While the nuclear charge distribution for a spin- $\frac{1}{2}$ nucleus is spherical, the distribution is uneven for a quadrupolar nucleus. The reorientation of the nucleus therefore affects the energy of the nucleus and the electric interaction of the nucleus with its surrounding becomes important, when dealing with a quadrupolar nucleus. Classically, the interaction betwren a charge distribution $\varrho(\vec{r})$ and an electric field potential $V(\vec{r})$ is

$$
E_{Q}=\int_{1} \varrho(\vec{r}) \mid i(\vec{r}) d \vec{r}
$$

integrated over the thrce-climensional space. Since $V(\vec{r})$ often takes a quite complex form dependent on the spatial distribution of all the electronic and nuclear charges, a Taylor series expansion is used inslead lo simplify Eqn 2.17.

$$
V(\vec{r})=V(0)+\left.\sum_{x=x \cdot y,:=} a \frac{\partial V}{\partial n}\right|_{\vec{r}=0}+\left.\frac{1}{2 !} \sum_{\alpha, \beta=x, y, z} a \beta \frac{\partial^{2} V}{\partial \alpha \partial \beta}\right|_{\vec{r}=0}+\ldots
$$

The expansion is about the contor of mass of the mucleus, which coincides with the center of charge of the muclous. Substituling Equ 2.18 into Eqn 2.17 yields the following expression.

$$
E_{Q}=Z e V(0)+\left.\sum_{n=x, y, z} P_{r r} \frac{\partial V}{\partial a}\right|_{r=0}+\left.\frac{1}{2 !} \sum_{\alpha, \beta=r, y, z} Q_{\alpha \beta}^{\prime} \frac{\partial^{2} V}{\partial \alpha \partial \beta}\right|_{r=0}+\ldots
$$


Here, $Z e$ is the total charge clensity of a mucleus, which is constant and of little interest; the $\alpha$ component of the clectric dipole moment

$$
P_{\alpha}=\int_{V} \alpha \varrho(\vec{r}) d \vec{r}
$$

equals to zero since the center of mass and center of charge for a nucleus coincide. The third term is the product of two second-rank tensors. The $\alpha \beta$ component of the electric quadrupole moment $Q_{n i}^{\prime}$ is clefined below.

$$
Q_{\alpha \beta}^{\prime}=\int_{1} \alpha \beta \rho(\vec{r}) d(\vec{r})
$$

The $\alpha \beta$ components of the clectric field gradient. (FFG) tensor is defined as

$$
V_{n ; 3}=\left.\frac{\partial^{2} V^{\prime}}{\partial \cap \partial \beta}\right|_{\vec{r}=0} .
$$

It is more convenient to express the EFG tensor in its principal axis system (PAS) with its three eigenvalues $V_{x x}, h_{y}$ and $V_{z z}$. These eigenvalues fulfill the following relationship.

$$
|| z z|\geq| \operatorname{ry}|\geq||x x|
$$

Since the EFG tensor is I raceless, 1 wo paramelors (eq and $\eta_{Q}$ ) are actually enough to define it unicyucly.

$$
\begin{aligned}
& \mu=1 \% \% \\
& \eta_{\varphi}=\frac{I_{X X}-I_{Y} y}{V_{y}}
\end{aligned}
$$

The second parameter $\eta_{Q}$ is the asymumetric parameler of the clectric field gradient and takes a value between 0 and 1. This parameter reflects the local symmetry around the nucleus, and c(puals to \%ro when fles surrounding of the nucleus is axial symmetric. 
The derivation of the quantum mechanical Hamiltonian for a quadrupolar nucleus then becomes quite complicated $[4,6]$, but the final result has the following form.

$$
H_{Q}=\hbar \omega_{Q} \sum_{m=-2}^{2} A_{2,-m}^{Q} T_{2 m}^{Q}
$$

where

$$
\omega_{Q}=\frac{c q \cdot e Q}{2 J(2 I-1) h}=\frac{e^{2} q Q}{2 \Gamma(2 I-1) \hbar} .
$$

The nuclear quadrupole moment. $e Q$ is derived from $Q^{\prime}$ in Eqn 2.21. A quadrupolar coupling constant $C_{Q}$ is now introduced to report the coupling between the nuclear quadrupole moment, and the electric field gradient.

$$
C_{Q}=\frac{e^{2} q Q}{\hbar}
$$

The different spin tensor components in Eqn 2.26 have the following meanings.

$$
\begin{aligned}
T_{20}^{Q} & =\frac{1}{\sqrt{6}}\left(3 I_{0}^{2}-I^{2}\right) \\
T_{2 . \pm 1}^{Q Q} & =\frac{1}{\sqrt{2}}\left(I_{0} I_{ \pm}+I_{ \pm} I_{0}\right) \\
T_{2 . \pm 2}^{Q} & =I_{ \pm}^{2}
\end{aligned}
$$

The spatial tensor component.

$$
f_{2 m}^{Q}=\sum_{n=-2}^{2} D_{n m}^{(2)}\left(\alpha^{Q}, \beta^{Q}, \gamma^{Q}\right) \rho_{2 n}^{Q}
$$

is expressed in the laboratory frame. The spalial lensor $\rho^{Q}$ in the quadrupole principal axis frame (PAS) is derfincel as

$$
\begin{aligned}
\rho_{20}^{Q} & =\sqrt{\frac{3}{2}} \\
\rho_{2 . \pm 1}^{Q} & =0 \\
\rho_{2 . \pm 2}^{Q} & =\eta_{Q} / 2 .
\end{aligned}
$$


These two tensors relatce to each ol.hor through a gencral rotation with three Euler angles $\alpha^{Q}, \beta^{Q}$ and $\gamma^{Q}$ between the two frames.

The magnitude of quadrupolar interaction is often in the megahertz range, and quadrupolar interaction dominates all other nuclear spin interactions except for the Zeeman interaction. Perturbative treatment for this interaction needs to be carried out to the second-order to fully account for its influence on the NMR spectra. On the olher hand, $C_{Q}$ and $\eta_{Q}$ contain invaluable inlormation about the local geometry around the nucleus that experimental extraction of them has proved essential in the determination of steructure and dymamics of many classes of materials.

\subsubsection{Chemical Shift Interaction}

Chemical shift interaction is a magnetic interaction deriving from the coupling between the nuclear dipole moment and the local magnetic field created by the motions of the surrounding clectrons. 'The Ilamiltonian is expressed here in the laboratory frame using a spherial tousor representation.

$$
H_{c s}=h_{1}\left(\cdot l_{00}^{(s)} T_{00}^{(s)}+\delta_{(s)} \sum_{m=-2}^{2}(-1)^{m} \cdot A_{2 m}^{c s} T_{2,-m}^{c s s}\right)
$$

In this equation, the spin space lonsors are given bolow in Leqn 2.37 to Equ 2.44 .

$$
\begin{aligned}
& T_{00}^{(S)}=B_{0} l_{0} \\
& T_{20}^{(S)}=\sqrt{\frac{2}{3}} B_{0} I_{0} \\
& T_{2 . \pm 1}^{(S)}=\frac{1}{\sqrt{2}} B_{0} I_{ \pm} \\
& T_{2 . \pm 2}^{r .3}=0
\end{aligned}
$$

Similar to the quadrupols asse it is more comenient to define chemical shift parameters in its principal axis systom (P:LS). The three eigrmalues of the tensor 


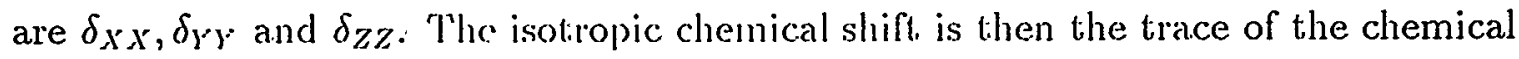
shift tensor.

$$
\delta_{i s o}=\frac{\delta_{X X}+\delta_{Y \gamma}+\delta_{Z Z}}{3}
$$

The assignment of $\delta_{X X}, \delta_{Y Y}$ and $\delta_{Z Z}$ needs to fulfill the following condition.

$$
\left|\delta_{Z Z}-\delta_{i s o}\right| \geq\left|\delta_{Y Y}-\delta_{i s o}\right| \geq\left|\delta_{X X}-\delta_{i s o}\right|
$$

The chemical shift anisotropy (CSA) and asymmetric parameter are defined by

$$
\begin{aligned}
& \delta_{C S}=\frac{3}{2}\left(\delta_{Z Z}-\delta_{i s i}\right) \\
& \eta_{C S}=\frac{\delta_{X X}-\delta_{Y Y}}{\delta_{Z Z}-\delta_{i s i}}
\end{aligned}
$$

where $\delta_{C S}$ characlerizes the size of CSA and $\eta_{C S}$ reflects the local symmetry around the nucleus.

Using the above clefinitions. the spatial tensor components $\rho_{i j}^{C S}$ in the chemical shift PAS are described as

$$
\begin{aligned}
& \rho_{00}^{c s}=\delta_{i s i} \\
& \rho_{20}^{c s}=\sqrt{\frac{3}{2}} \\
& \rho_{2 . \pm 1}^{c s}=0 \\
& \rho_{2 . \pm 2}^{c s}=\eta_{c s} / 2 .
\end{aligned}
$$

The spatial tensor $A^{c s}$ in the laboralory frame is obtained from the PAS frame tensor $\rho^{C S}$ through a gencral rotalion.

$$
-f_{l m}^{(S)}=\sum_{n=-1}^{l} D_{n m}^{(l)}\left(\alpha^{C S S}, \beta^{C s s}, \gamma^{C S s}\right) \rho_{l n}^{C S S}
$$


Here, $\alpha^{C S}, \beta^{c s}$ and $\gamma^{(S)}$ are Euler angles berwen the CSA PAS and the laboratory frame.

Chemical shift anisotropy is usually in the range of kilohertz and is more conveniently reported in the units of parts por million (ppm). Perturbative treatment of this interaction only necels to be clone to the first-order. Like $C_{Q}$ and $\eta_{Q}, \delta_{i s o}, \delta_{C S}$ and $\eta_{C S}$ probe the local structure and cynamics near to the nucleus that a series of NMR applications are solely dependent on the accurate measurement of these parameters.

\subsubsection{Dipolar Interaction}

Dipolar Interaction (1)!) Lotween two spins $I_{i}$ and $I_{j}$ is described by following equation.

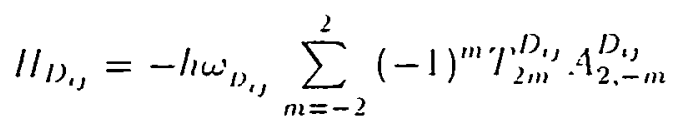

Here, $\omega_{D_{1}}$ is the magnitude of the dipolar interaction that ranges from zero to tens of kilohertz for diflorent spin pairs.

$$
\omega_{\nu_{1,}}=\frac{\hat{1}_{1} \hat{i}_{j}}{r_{i, 1}^{3}}
$$

The spin tensor components are given by

$$
\begin{aligned}
& Y_{20}^{D_{1}}=\frac{1}{\sqrt{6}}\left(3 I_{\% .2} I_{\%, 1}-I_{1} \cdot I_{.1}\right) \\
& \%_{2 . \pm 1}^{L_{2,}}=\frac{\sqrt{2}}{2}\left(I_{\%, 1} I_{ \pm .,}+I_{ \pm .,} I_{z . J}\right) \\
& l_{2 . \pm 2}^{l_{1,3}}=l_{ \pm .1} l_{ \pm .1}
\end{aligned}
$$

The lab frame tensor.$^{1 / 2}$ is delined based on l(nusor $\rho^{1)_{2}}$ in the dipolar principal axis system (PAS). The conversion beneru the two tensors involves only one Euler 
angle $\left(\beta^{D_{13}}\right)$ since only the $z$-axis of the dipolar PAS frame is fixed.

$$
A_{2 m}^{D_{i j}}=\sum_{n=-2}^{n=2} D_{n m}^{(2)}\left(0, \beta^{D_{\imath \jmath}}, 0\right) \rho_{2 n}^{D_{i \jmath}}
$$

where

$$
\begin{aligned}
& \rho_{20}^{D_{1 j}}=\sqrt{\frac{3}{2}} \\
& \rho_{2, \pm 1}^{D_{i j}}=0 \\
& \rho_{2, \pm 2}^{D_{1 j}}=0 .
\end{aligned}
$$

The strong dependence of $w_{D_{1}}$, on the distance between the two spins makes the dipolar interaction a unicue probe on internuclear distances. This thesis, however, will not consider dipolar interaction in much detail. The readers can consult with other references to see how the dipolar in teraction is utilized to enhance NMR signal $[13,14]$, to do spectral oditing [15] and to establish connectivities among complex spin networks $[5,16]$.

\subsection{Perturbative Treatment of Spin Hamiltonians}

We now start to look at how the internal spin interactions affect the NMR spectra using perturbation theory. As montioned before. litst-order perlurbative trealment is needed for all hihes of the incractions (CSA, QI and DI), while second-order expansion is also necled when doaling with the quadrupolar interaction. As long as the first-order perturbation theory is concerned. only the sccular part of the Hamiltonians that commute with the \%eman Ilamiltonian remains and all other terms can be safely dropped. 


\subsubsection{Static Sample}

The part of the chomical shift. Ilamiltonian that commutes with the Zeeman Hamiltonian is given below.

$$
\begin{aligned}
H_{C S, S} & =h \gamma\left(. A_{00}^{C S S} T_{00}^{C S S}+\delta_{C S S} A_{20}^{C S S} T_{20}^{C S S}\right) \\
& =h \omega_{0} \delta_{2 s o} I_{0}+h \gamma \delta_{C S} T_{20}^{C S S} A_{20}^{C S S} \\
& =h \omega_{0}\left(\delta_{i s o} l_{0}+\sqrt{\frac{2}{3}} \delta_{C S S} l_{0} \cdot A_{20}^{C S}\right)
\end{aligned}
$$

In terms of the encrgy correction, Fyn 2.9 is used and

$$
\begin{aligned}
& \omega_{m \leftrightarrow n}^{c s}=\left(<\operatorname{lm}\left|H_{c s}\right| \ln >-<\ln \left|\|_{(s)}\right| \ln >\right) / h \\
& =(m-n) \omega_{00}\left(\delta_{i s u}+\sqrt{\frac{2}{3}} \delta_{C s} \cdot H_{20}(\dot{s})\right.
\end{aligned}
$$

The factor $(m-11)$ lice suggests lhat 1 he $3 / 2 \leftrightarrow-3 / 2$ transition would have a chemical shift tripled that of the $1 / 2 \leftrightarrow-1 / 2$ l.ansilion.

Truncation of the guadrupolar Ilamiltonian to the first-order gives

$$
I_{Q}=\frac{h \omega_{Q}}{\sqrt{6}} \cdot 1_{20}^{Q}\left(3 I_{0}^{2}-I(I+1)\right)
$$

The first-order cuergy correction 10 stats $\mid / m>$ is again calculated by inserting this equation into Fign 2.9.

$$
\left.<|m|\left|I_{Q}\right| / m\right\rangle=\left.\frac{\operatorname{l\omega } Q}{\sqrt{6}} \cdot\right|_{20} ^{Q}\left(3 m^{2}-I(l+1)\right)
$$

It is insighteful to work out the conerge differencess for varions transitions of a halfinteger quadrupolar mucleus.

$$
\begin{aligned}
& \left.\Delta E_{m \leftrightarrow m-1}^{1 Q}=\frac{3 h \omega_{Q}}{\sqrt{6}} \cdot 1_{210}^{Q}(2) m-1\right) \\
& \Delta I_{m \leftrightarrow-m}^{1 Q}=0
\end{aligned}
$$


For the $m \leftrightarrow m-1$ transition with $m \neq \frac{1}{2}$, the correction is in the order of $\omega_{Q}$ (megahertz) and can hardly be observed in most experiments (for opposite examples, see $[17,18,19])$. The most commonly observed central transition $(1 / 2 \leftrightarrow-1 / 2$ transition), together with other symmetric multiple-quantum transitions ( $m \leftrightarrow-m$ transitions), is however, not affected to the first-order by the quadrupolar interaction. Second-order perturbation theory is thus used, and the final results are described below [20].

$$
\begin{aligned}
\Delta E_{m \leftrightarrow-m}^{2 Q}= & \frac{\hbar \omega_{Q}^{2}}{\omega_{0}}\left(\left(A I(I+1)-\operatorname{sm}^{2}-1\right) A_{21}^{Q} A_{2,-1}^{Q}+\right. \\
& \left.\left(2 I(I+1)-2 m^{2}-1\right) A_{22}^{Q} A_{2,-2}^{Q}\right)
\end{aligned}
$$

This equation reduces to the more commonly used expression for the central transition.

$$
\Delta E_{1 / 2 \leftrightarrow-1 / 2}^{2 Q}=\frac{h \omega_{Q}^{2}}{2 \omega_{0}^{2}}(I(I+1)-3 / 4)\left(2 A_{21}^{Q} A_{2,-1}^{Q}+A_{22}^{Q} A_{2,-2}^{Q}\right)
$$

Figure 2.2 shows schematically how the energy levels of a spin $I=\frac{3}{2}$ nucleus are shifted by the quadrupolar intcraction. Wilhout the quadrupolar interaction, four $(2 I+1)$ equally spaced energy levels are observed. The first-order quadrupolar interaction changes the encrgy of each level by an amount in the order of the quadrupolar interaction (megahcrt.z). IIowerer, the $\mathrm{m}$ and $-\mathrm{m}$ energy levels are shifted in the same direction, and the $m \leftrightarrow-m$ transition frequency is not affected to the firstorder. The second-order clladrupolar interaction. however, does affect the transition frequencies and the frepurency shifts are described by ligns 2.(j5) and 2.66 .

\subsubsection{Spinning Samples}

To yield better resolution, most solid-state NMR experiments are carried out under spinning condition. Sample spinning introduces extra rotations in the real 


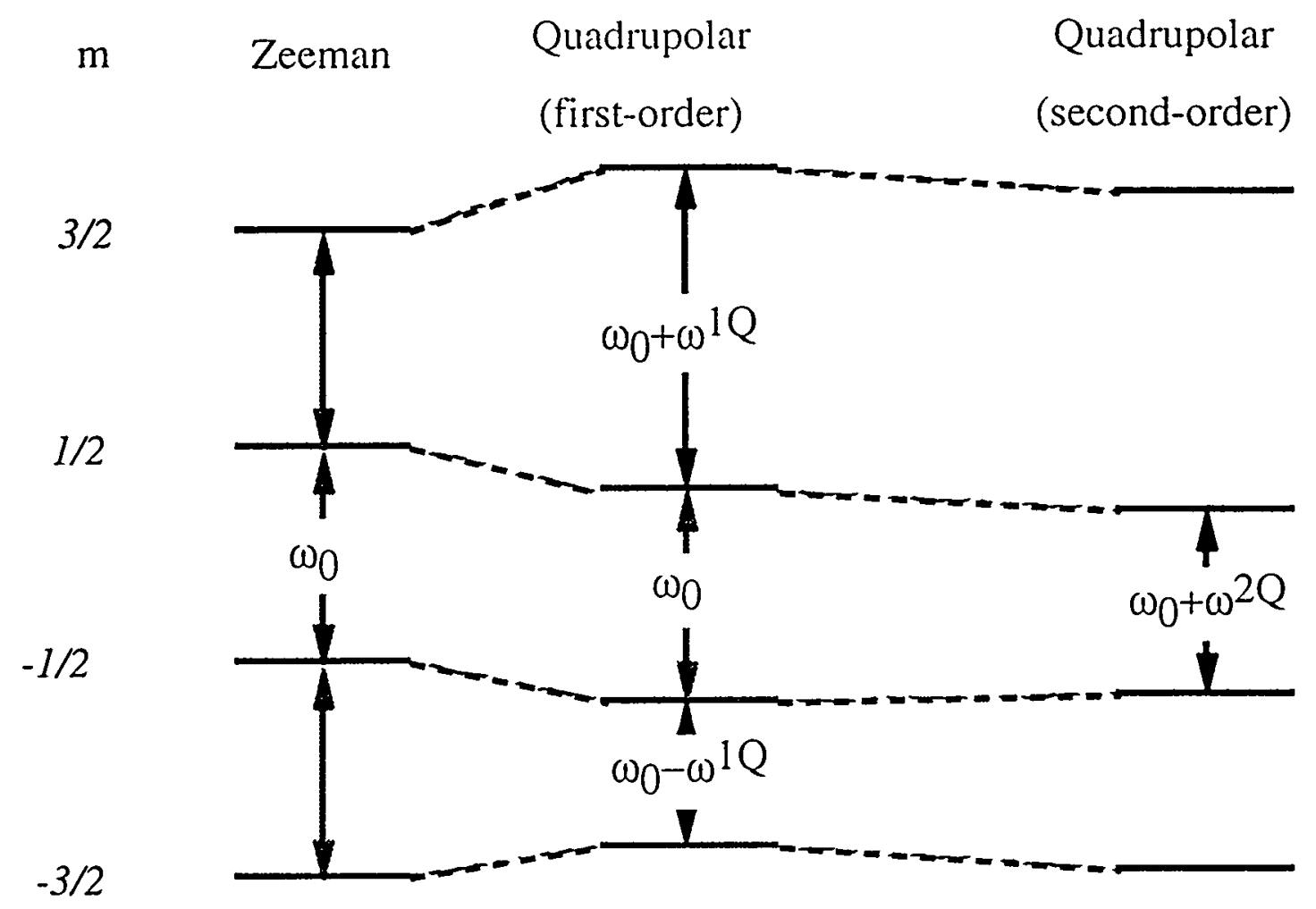

Figure 2.2: Zeeman, first- and second-order energy' splittings for $I=\frac{3}{2}$ nucleus.

space (but does not change the spin space tensors), so the format of the energy correction expressions is not changed (Eqn 2.60 and Eqn 2.65), except that the spatial tensors $A^{Q}$ and $A^{c} s$ now involve another rotation in the real space.

Figure 2.3 demonst rales the tansformalions we need to proform on a spatial tensor $A^{\lambda}$ in a rotating sample. The lensor is first lansformed from its principal axis frame (PAS) to the rotor lanc, and then to the laboratory frame. The Euler angles involved in the two t.manshormat ions are $(0,6, \gamma)$ and $(\omega, 1,0,0)$ respectively. Here $\omega_{r}$ is the spinning speed and 0 is the angle bewern the spinner axis and the static field $B_{0}$. According to lixy 2.2 .

$$
\cdot H_{l m}^{l}=\sum_{l=-l}^{l} \sum_{n=-l}^{l} D_{n k}^{(l)}(0,, \beta, \gamma) D_{k+m}^{(l)}\left(\omega_{r} l, 0,0\right) \rho_{l n}^{l} .
$$

As a comparison, here is only one rotat ion involved in the static case (see Egn 2.32 


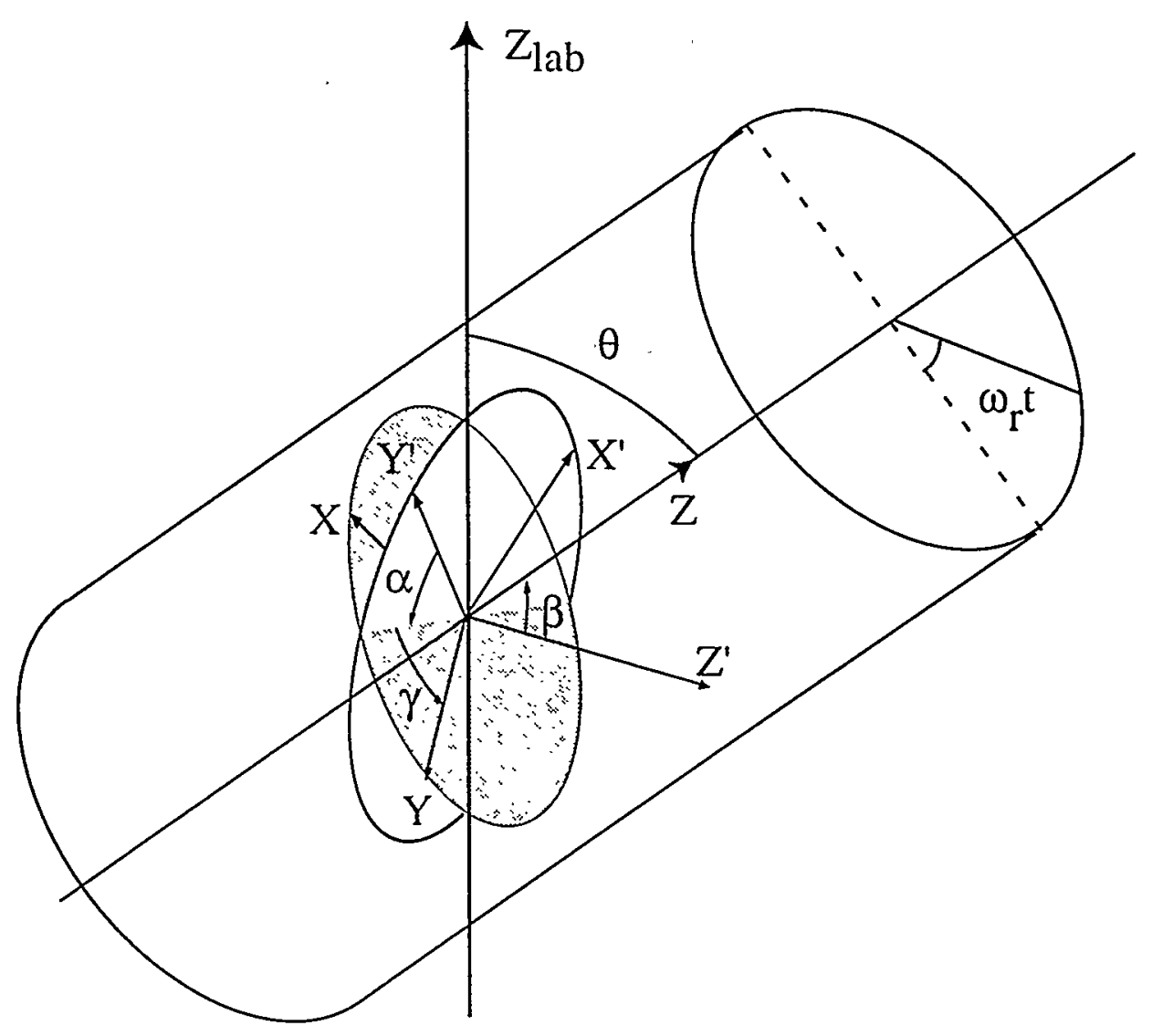

PAS Frame $\stackrel{(\alpha, \beta, \gamma)}{\longrightarrow}$ Rotor Frame $\stackrel{\left(\omega_{\mathrm{r}} \mathrm{t}, \theta, 0\right)}{\longrightarrow}$ Lab Frame

Figure 2.3: PAS to rotor to lab frame. The Euler angles used in the transformation are indicated.

and Eqn 2.49). The above expression is now time-dependent and can be written as the sum of the time-indepcndent and time-dependent terms. The time-dependent terms result in extra peaks (sidebands) in an NMR spectrum, and will not be discussed in detail [21, 22]. Here, wr consider only the lime-independent $(k=0)$ terms, which suffice for the derivation of hightresolution NMR theory.

For chemical shift intcraction. the important spatial tensor is $A_{20}^{C S}$ (Eqn 2.60). 
According to Eqp 2.6\%.

$$
A_{20}^{C S S}=\sum_{k=-2}^{2} D_{k \cdot 0}^{(2)}\left(\omega_{r}, l, 0,0\right) \sum_{n=-2}^{2} D_{n k}^{(2)}\left(\alpha^{C S}, \beta^{C S S}, \gamma^{C S}\right) \rho_{2 n}^{C S} .
$$

The $k \neq 0$ terms in the summation can be dropped since they are time-dependent. The remaining $k=0$ terms give the following expression for $A_{20}^{C S S}$.

$$
A_{20}^{C S S}=\sqrt{\frac{3}{2}} P_{2}(\cos \theta)\left(\frac{3 \cos ^{2} \beta^{c S}-1}{2}+\frac{\eta(s)}{2} \sin ^{2} \beta^{C s s} \cos 2 \alpha^{C S}\right)
$$

Here, $P_{2}(\cos \theta)$ is the sccond-order Legendre polynomial.

$$
P_{2}(\cos \theta)=\frac{3 \cos ^{2} \theta-1}{2}
$$

A similar result is obtained for the first-oreler quadrupolar interaction.

$$
A_{20}^{1 Q}=\sqrt{\frac{3}{2}} P_{2}(\cos \theta)\left(\frac{3 \cos ^{2} \beta^{Q}-1}{2}+\frac{\eta_{Q}}{2} \sin ^{2} \beta^{Q} \cos 2 \alpha^{Q}\right)
$$

The expressions for a static sample can be dorived by setting $P_{2}(\cos \theta)=1$ (or $\left.0=0.0^{\circ}\right)$. Applying Equ 2.69) 1.0 Equ 2.60, a more often used version of the chemical shift frequency is writicn as.

$$
\omega^{c r}=\omega_{\text {iso }}+A_{2}^{(r)}\left(\alpha^{c i s} \cdot j^{\cos }\right) P_{2}(\cos \theta)
$$

where

$$
\omega_{i s,}=\omega_{(1)}^{\prime} \delta_{\text {LSt }}
$$

and

$$
H_{2}^{(\cdot)}=\sqrt{\frac{2}{3}} \delta_{(} \cdot A_{20} \cos / P_{2}(\cos \theta)
$$

Eqn 2.72 contains an isot ropic term that is independent of crystalline orientation, and an anisotropic term that is dependent on the crystalline orientation. The latter needs 
to be averaged out if high-resolution is desired. Similar expression can be obtained for the first-order quadrupolar interaction.

For the second-order quadrupolar interaction, the product of two second-rank tensors $\left(A_{2 m}^{Q} A_{2,-m}^{Q}\right)$ needs to be expancled. There are two ways of calculating the expansion. The first one works well when the time-dependent terms in the expansion are not important and can be dropped (which is the fast-spinning case). The tensor product is first explicitly written out as follows.

$$
\begin{aligned}
A_{2 m}^{Q} A_{2,-m}^{Q}= & \sum_{l, n=-2}^{2} D_{n l}^{(2)}\left(\alpha^{Q}, \beta^{Q}, \gamma^{Q}\right) D_{l n}^{(2)}\left(\omega_{r} t, 0,0\right) \rho_{2 n}^{Q} \times \\
& \sum_{k, p=-2}^{2} D_{p k}^{(2)}\left(\alpha^{Q}, \beta^{Q}, \gamma^{Q}\right) D_{k,-m}^{(2)}\left(\omega_{r} . t, 0,0\right) \rho_{2 p}^{Q}
\end{aligned}
$$

Only the $l=k=0$ lerms in the above equation are time-independent and are separated out.

$$
\begin{aligned}
A_{2 n 2}^{Q} A_{2,-m}^{Q}= & \sum_{n=-2}^{2} D_{n 0}^{(2)}\left(\alpha^{Q}, \beta^{Q}, \gamma^{Q}\right) D_{0 m}^{(2)}\left(\omega_{r} t, 0,0\right) \rho_{2 n}^{Q} \times \\
& \sum_{p=-2}^{2} D_{p 0}^{(2)}\left(\alpha^{Q}, \beta^{Q}, \gamma^{Q}\right) D_{0,-m}^{(2)}\left(\omega_{r} t, 0,0\right) \rho_{2 p}^{Q}
\end{aligned}
$$

For the central transition, the cxpression $2 A_{21}^{Q} A_{2,-1}^{Q}+A_{22}^{Q} A_{2,-2}^{Q}$ expands as below.

$$
2 A_{21}^{Q} \cdot A_{2,-1}^{Q}+A_{2,2}^{Q} \cdot 1_{2,-2}^{Q}=\frac{1}{\delta} \sum_{1, j=0}^{2} a_{i j}^{\prime}\left(\eta_{Q}\right) \cos 2 i \alpha^{Q} \cos 2 j \beta^{Q}
$$

where

$$
a_{i, 1}^{\prime}=a_{i, j}^{(0)}+a_{i, j}^{(2)} P_{2}(\cos \theta)+a_{i, j}^{(\cdot 1)} P_{.1}(\cos \theta)
$$

Here,

$$
P_{1}(\cos \theta)=\frac{1}{8}\left(35 \cos ^{4} \theta-30 \cos ^{2} \theta+3\right)
$$


is the 4 th-order Legendre polynomial. 'The $\eta_{Q}$-dependent $a_{i j}$ elements are given in table $2.2[23,24]$. Using these parametors, static and MAS spectra of quadrupolar nuclei can be simulated (simulaling the sidebands requires extra effort).

\begin{tabular}{ccccc}
\hline$i$ & $j$ & $a_{i j}^{(0)}$ & $a_{i j}^{(2)}$ & $a_{i j}^{(4)}$ \\
\hline 0 & 0 & $-\frac{12}{5}\left(1+\frac{1}{3} \eta_{Q}^{2}\right)$ & $-\frac{12}{7}\left(1-\frac{1}{3} \eta_{Q}^{2}\right)$ & $\frac{81}{1120}\left(18+\eta_{Q}^{2}\right)$ \\
0 & 1 & 0 & $-\frac{36}{7}\left(1-\frac{1}{3} \eta_{Q}^{2}\right)$ & $\frac{9}{56}\left(18+\eta_{Q}^{2}\right)$ \\
0 & 2 & 0 & 0 & $\frac{9}{32}\left(18+\eta_{Q}^{2}\right)$ \\
1 & 0 & 0 & $\frac{2 \cdot 1}{7} \eta_{Q}$ & $\frac{81}{56} \eta_{Q}$ \\
1 & 1 & 0 & $-\frac{2 \cdot 1}{7} \eta_{Q}$ & $\frac{27}{14} \eta_{Q}$ \\
1 & 2 & 0 & 0 & $-\frac{27}{8} \eta_{Q}$ \\
2 & 0 & 0 & 0 & $\frac{27}{32} \eta_{Q}^{2}$ \\
2 & 1 & 0 & 0 & $-\frac{9}{8} \eta_{Q}^{2}$ \\
2 & 2 & 0 & 0 & $\frac{9}{32} \eta_{Q}^{2}$ \\
\hline
\end{tabular}

Table 2.2: Coefficients in the anisotropic cosine expansion for second-order quadrupolar correction.

Substituting Fyn 2.Tt into Eqn 2.66, the energy splitting due to second-order quadrupolar interaction is

$$
\Delta E_{1 / 2 \leftrightarrow-1 / 2}^{2 Q}=\frac{h_{Q} C_{Q}^{2}(I(I+1)-3 / 4)}{1 /^{2}(2 /-1)^{2} \omega_{u}}\left(2 A_{21}^{Q} \cdot A_{2,-1}^{Q}+A_{22}^{Q} A_{2,-2}^{Q}\right),
$$

which after rearangenent. loads to the following form for the transition frequency.

$$
\omega^{2 Q}=\omega_{1, s u}^{2 Q}+.1_{2}^{Q}\left(a^{Q}, 3^{Q}\right) P_{2}^{2}(\cos \theta)+. I_{1}^{Q}\left(a^{Q}, \beta^{Q}\right) P_{4}(\cos \theta)
$$

Here,

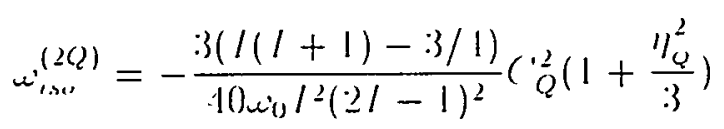

is the isotropic seconcl-order quadrupolar shift. The last. fwo toms, being anisotropic, depend on the crystalline oricntation and the relative oriontation between the rotor axis and the laboratory frame z-axis. 'These two terms are lo be averaged out when high-resolution spectira are desired. 
If Eqn 2.65 instead of Pqn 2.66 is combined with Eqn 2.77, the second-order quadrupolar frequency shift for the $m \leftrightarrow-m$ transition is obtained.

$\omega_{m \leftrightarrow-m}^{2 Q}=C_{0}(I, m) \omega_{i s o}^{2 Q}+C_{1}(I, m) A_{2}^{Q}\left(\alpha^{Q}, \beta^{Q}\right) P_{2}(\cos 0)+C_{2}(I, m) A_{4}^{Q}\left(\alpha^{Q}, \beta^{Q}\right) P_{4}(\cos \theta)$

Compared to Eqn 2.81, threc constants dependent on the transition and the spin quantum number are introduced. These values are shown in table 2.3.

\begin{tabular}{ccccc}
\hline$I$ & $m$ & $C_{0}$ & $C_{1}$ & $C_{2}$ \\
\hline $3 / 2$ & $1 / 2$ & 1 & 1 & 1 \\
& $3 / 2$ & -3 & 0 & $-21 / 27$ \\
$5 / 2$ & $1 / 2$ & 1 & & \\
& $3 / 2$ & $6 / 8$ & 1 & 1 \\
& $5 / 2$ & $-50 / 8$ & $-20 / 32$ & $-150 / 72$ \\
& & & & \\
$7 / 2$ & $1 / 2$ & 1 & 1 & 1 \\
& $3 / 2$ & $27 / 15$ & $144 / 60$ & $303 / 135$ \\
& $5 / 2$ & -1 & 2 & $165 / 135$ \\
& $7 / 2$ & $-1 \cdot 17 / 15$ & $-168 / 60$ & $-483 / 185$ \\
& & & & \\
$9 / 2$ & $1 / 2$ & 1 & 1 & 1 \\
& $3 / 2$ & $51 / 24$ & $252 / 96$ & $546 / 216$ \\
& $5 / 2$ & $30 / 24$ & $300 / 96$ & $570 / 216$ \\
& $7 / 2$ & $-8 \cdot 1 / 24$ & $168 / 96$ & $84 / 216$ \\
& $9 / 2$ & $-3.2 \cdot 1 / 24$ & $-216 / 96$ & $-1116 / 216$ \\
\hline
\end{tabular}

Table 2.3: Coclficionts $C_{0} \cdot C_{1}$ and $C_{2}$ in Equ 2.83.

Another way of expranding the product of second-rank tensors takes the advantage that the product of two second-rank fonsors is a sum of rank $0,2,4$ tensors.

$$
A_{2 m}^{Q}-A_{2,-m}^{Q}=\sum_{l=0.2 .4}<10 \mid 22 m,-m>a_{l l}
$$


In this equation, $a_{t 0}$ is the tortsor clement of an lth-mank tensor.

$$
a_{l 0}=\sum_{n=-l}^{l} D_{n 0}^{(l)}\left(\omega_{r} l, 0,0\right) \sum_{k=-l}^{l} D_{k n}^{(l)}\left(\alpha^{Q}, \beta^{Q}, \gamma^{Q}\right) \sigma_{l k}
$$

Here

$$
\sigma_{l k}=\sum_{J=-2}^{2}<l k \mid \cdot 2 \cdot j, k-j>\rho_{2 j} \rho_{2, k-j}
$$

and $\langle l 0| 22 m,-m>$ and $<|k| 22 j, k-j>$ are Gilebsh-Gordon coefficients [12].

Of all the terms in Bgn 2.85. only the $n=0$ components are time-independent. Neglecting all the lorms with $n \neq 0$.

$$
A_{2 m}^{Q} A_{2,-m}^{Q}=\sum_{l=0.2,1} d_{00}^{(l)} \sum_{l=-l}^{l}<|0| 2 \cdot 2 m,-m>D_{k \cdot 0}^{(l)}\left(\alpha^{Q}, \beta^{Q}, \gamma^{Q}\right) \sigma_{l k}
$$

Notice that

$$
d_{\mathrm{ij0}}^{(l)}(\cos \theta)=P_{l}(\cos \theta)
$$

Eqn 2.81 is reached after inserting Equ 2.87 into Eqn 2.80.

\subsection{Evolution of Spin System}

After a brief discussion of the cigenstates and cigenvalues of a single spin, we now start to look at how these rigenstales and rigemalues crolve in an NMR experiment. The evolution corresponds lo rolations in the spin space, and is better described with the conceptss of density matrix and densit! operator introduced as follows [11, 12].

\subsubsection{Density Operator}

For a given spin coscomble. if all of the spins are in the same state (pure state), described by the state function $\mid \Psi(l)>$ in an orlhomormal basis $\{\mid I n>\}$ with coeffi- 
cients $C_{m}(t)$, the densily: operalor $\rho(t)$ is defined by:

$$
\rho(t)=|\Psi(t)><\Psi(t)|=\sum_{m, n=-I}^{I} C_{m}(t) C_{n}^{*}(t)|I m .><I n|
$$

If on the other hand, not all of the spins are in the same state (mixed state), the density operator is described by the ensemble average over all the spins.

$$
\rho(t)=\overline{|\Psi(t)><\Psi(t)|}=\sum_{m, n=-I}^{I} \overline{C_{m}^{\prime}(t) C_{n}^{*}(t)}|I m .><I n|
$$

The clensity matrix $A$ is the mal.rix representation of the density operator, and is used to facilitate the numerical calculation of the evolution of the density operator.

$$
A_{m n}(l)=\overline{C_{m}(l) C_{n}^{*}(t)}
$$

The equation of molion of the clensity operator is described by the Liouville-von Neumann equation

$$
\frac{d}{d l} \rho(l)=-i[H(l), \rho(l)]
$$

whose solution is given by:

$$
\rho(l)=l l(l) \rho(0) l(t)^{-1}
$$

Here,

$$
\left.U^{\prime}(l)=T c \cdot x\right)\left(-i \int_{0}^{l} H\left(I^{\prime}\right) d l^{\prime}\right)
$$

and $T$ is the Dyson timc-ordering operator [25]. Wihon $H(1)$ is lime-independent, the evolution of the density matrix can he simply represented as

$$
\rho(l)=e^{-i l l \prime} \rho(0) e^{i \prime \prime \prime}
$$

In a real system where sample is spinning, $H$ is often time-dependent even in the rotating frame (the reforence frame that rotates around the laboratory $z$-axis at the 
Larmor frequency. In this liame, the effect of the static field on the NMR spectra is removed. It is also the NMR detection frame). The general approach to calculate the density matrix at time $/$ is to break up the time axis into small intervals, during each of which the Hamilionian is constant, (Figure 2.4). The density operator is then the cascaded evolutions in all the lime intervals.

$$
\rho(t)=e^{-i H_{n} t_{n}} e^{-i H_{n-1} t_{n-1}} \cdots c^{-i H_{1} t_{1}} \rho(0) c^{i H_{1} t_{1}} \cdots e^{i H_{n-1} t_{n-1}} e^{i H_{n} t_{n}}
$$

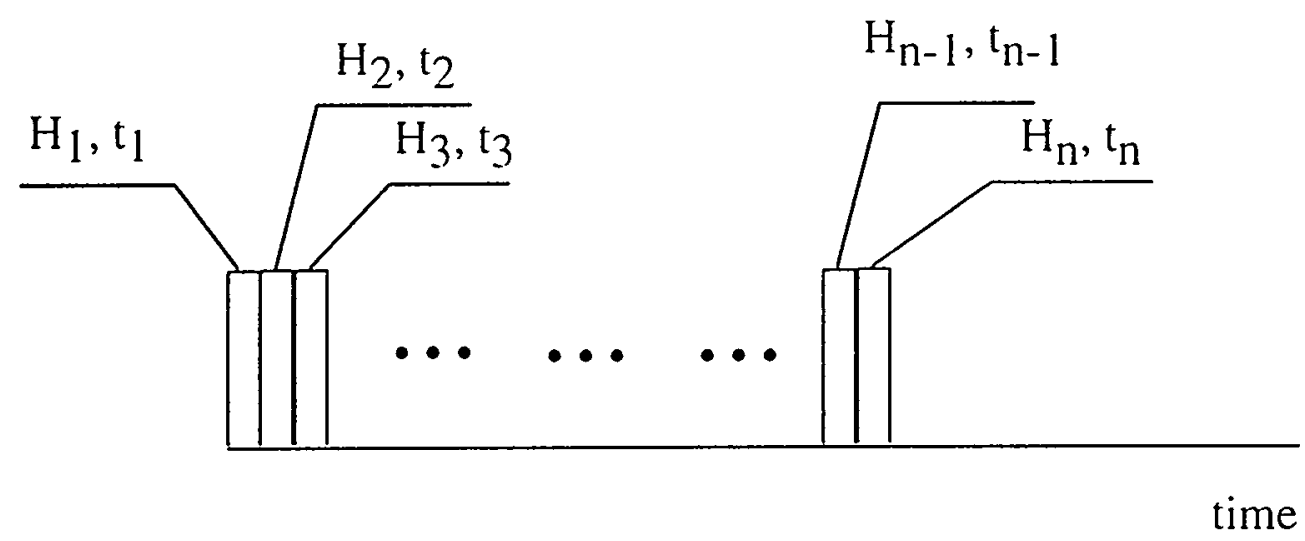

Figure 2.4: Treatment of a lime-dependent. Ilamiltonian. The time axis is divided into small intervals, during cacl, of which the spin Ilamilonian is time-independent.

The obscrvable in an NAll reporiment is $I_{+}$. and the detection is achieved by using a single coil capable of collecting data in quadrature (both the real and imaginary componculs of lhe signal). I'sing the density operator representation, the expectation value of the operator $I_{+}$is expersecel as

$$
\left\langle l_{+}\right\rangle=r \cdot\left(\rho(l) l_{+}\right)=\sum_{r=-1}^{\prime}\left\langle|r| \rho(l) l_{+} \mid l_{r}\right\rangle \text {. }
$$

$\left\langle I_{+}\right\rangle$is time-dependent and induces an olectric signal that is picked up by a detection coil. 'The detered signal is the froc induction decay (FID) of the system. 
In most experiments, the phase of the receiver $\left(\dot{\theta}_{r}\right)$ may be controlled to arbitrary accuracy in the computer, so the actual signal is clescribed as

$$
S(t)=e^{-i \phi_{r}}<I_{+}>=e^{-i \phi_{r}} \operatorname{Tr}\left(\rho(t) I_{+}\right)
$$

By inserting Eqn 2.90 into Eqn 2.97, we get.

$$
\begin{aligned}
\left\langle I_{+}\right\rangle & =\sum_{m, n, r=-l}^{I} \overline{C_{m}^{\prime}(t) C_{n}^{* *}(t)}<I r|I m><I n| I_{+}|I r\rangle \\
& =\sum_{m, n, r=-l}^{l} \overline{C_{m}^{\prime}(t) C_{n}^{*}(l)} \delta_{m, r} \delta_{n, r+1} \\
& =\sum_{n, m=-l}^{l} \overline{C_{m}^{\prime}(l) C_{n l}^{*}(l)} \delta_{n, m+1}=\sum_{m=-I}^{I} A_{m-1, m} .
\end{aligned}
$$

This suggests that only single-quantum transitions $(m \leftrightarrow m-1)$ are directly observable, whereas multiple-quantum transitions have to be detected indirectly. This also brings about the concept of coherence, which is specified by a variable number $p$, and corresponds to the off-diagonal elements $A_{m n}(m \neq n)$ in the density matrix. Usually, a non-zero off-diagonal clement in the clensity matrix means that there is a connection between the lwo encrgy levels. $\Lambda$ coherence phase factor then exists in the density matrix elcment, which crolves as the system cvolves. At equilibrium, however, only diagonal elements cxist and the densiby matrix is

$$
\rho(0)=\frac{\varepsilon^{-1 / z / k \cdot T}}{Z} .
$$

where $Z$ is a normalization profactor and $I_{Z}$ is the Zceman Hamiltonian. With $\mathrm{H}_{Z}$ being much smaller than $k T, \rho_{0}$ is approximated as

$$
\rho(0) \approx \frac{\operatorname{li\gamma } B_{0}}{(2 I+1) k T} I_{0} \rightarrow \rho(0)=I_{0}
$$


after dropping the constant prefactors. This gires the intial state of an NMR system. The linear dependence of $\rho(0)$ on $\gamma$ moans that a larger $\gamma$ often gives a larger population difference and stronger NMIR signal.

\subsubsection{Evolution Under RF pulses}

Radio frequency pulses of a well-defined length $(\tau)$, amplitude $\left(B_{1}\right)$ and phase $(\phi)$ are used to rotate nuclear spin states by crating and destroying coherences or changing populations of llo different energy levels. 'The strength of the pulse is defined by

$$
\omega_{1}=\gamma B_{1}
$$

The frequency $w$ of the pulse is often sot very close to the Larmor frequency $\omega_{0}$, so the Hamiltonian corresponds to the RF pulse is described as

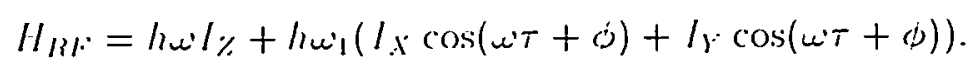

In the rotating frame. tho latrmor frefurncy no longer enters the cxpression,

$$
I_{R I}^{\prime f f}=h \Delta \omega / \%+h \omega^{\prime}\left(I_{X} \cos \phi+I_{Y} \cdot \sin \phi\right)
$$

and the pulse acts as if it is a static fichl in the transverse $x-y$ plane at an angle $\phi$ with respect to the $x$-ixis. Here. Jw includes the chomical shift contribution and the frequency offset in an experiment. Notice that this IIamiltonian is no longer time-dependent in the rotating frame, one can casily calculate the density operator at lime $l$.

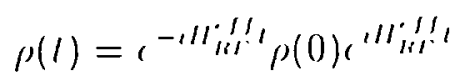

For a spin- $\frac{1}{2}$ nucless under Rl: irradiation with phase o. the evolution of different initial states is shown in lhe following equations.

$$
U_{R F}\left(I_{a}\right) I_{\%} l^{\prime}-1 / \cdot\left(I_{a}\right)=I_{\%} \cos \omega_{1} l+\sin \omega_{1} l\left(I_{x} \sin \theta-I_{1} \cdot \cos \phi\right)
$$




$$
\begin{aligned}
U_{R F}\left(I_{\phi}\right) I_{X}\left(I_{R F}^{-1}\left(I_{\phi}\right)=\right. & I_{X}\left(\cos ^{2} \phi+\sin ^{2} \phi \cos \omega_{1} l\right)+I_{Y} \frac{\sin 2 \phi}{2}\left(1-\cos \omega_{1} t\right) \\
& -I_{Z} \sin \phi \sin \omega_{1} t \\
U_{R F}\left(I_{\phi}\right) I_{Y} U_{R F}^{-1}\left(I_{\phi}\right)= & I_{Y}\left(\sin ^{2} \phi+\cos ^{2} \phi \cos \omega_{1} l\right)+I_{X} \frac{\sin 2 \phi}{2}\left(1-\cos \omega_{1} t\right) \\
& +I_{Z} \cos \phi \sin \omega_{1} t
\end{aligned}
$$

Here, $U_{R F}\left(I_{\phi}\right)=e^{-i l l / f f}$ and $\phi$ is the phase of the pulse. These equations are more useful when they are expressed in the spherical tensor basis set.

$$
\begin{aligned}
U_{R F} I_{0} U J_{R F}^{-1}= & I_{0} \cos \omega_{1} l+\frac{i \sin \omega_{1} t}{\sqrt{2}}\left(I_{+} e^{-i \phi}+l_{-} e^{i \phi}\right) \\
l_{R F} l_{ \pm} l:-1== & \frac{1}{2} l_{ \pm}\left(1+\cos \omega_{1} l\right)+\frac{i}{\sqrt{2}} l_{0} e^{ \pm i \phi} \sin \omega_{1} t \\
& -\frac{1}{2} l_{\mp} \epsilon^{ \pm 2 i \phi}\left(1-\cos \omega_{1} t\right)
\end{aligned}
$$

Some important points are worth noting:

I. A $90^{\circ}$ pulse $\left(\omega_{1} l=\pi / 2\right)$ generates a $90^{\circ}$ rotation in the spin space. It rotates $I_{0}$ into $x-y$ planc and creates both $I_{+}$and $I_{-}$coherences (we assign each coherence $I_{+}$(or $I_{-}$) a coherence number $1(-1)$, and give it a variable name p). 'The colierences after the pulse have well-defined phases. For example, a $90^{\circ}$. pulsc crates magnetization along the $-y$ direction.

II. When

$$
\left[H_{R H}^{S f J} \cdot \rho(0)\right]=0
$$

the densily matrix does not crolve since

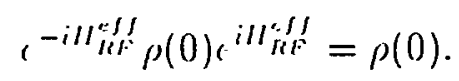


For example, after a $90^{\circ}$, pulse, if another pulse is applied along the $y$ direction, The donsity matrix does not evolve and the system is in a spin-locked stale. 'The second pulse is called a spin-locking pulse.

III. A single pulse causes the mixing of elements in the density matrix. For instance, if $\rho(0)=l_{+}$and another $R F$ pulse is applied for a time $t, \rho(t)$ is a mixlure of threc coherences: $+1,0$ and -1 . The change of coherence order under RF pulso is called coherence transfor.

IV. The phase factor $(\Delta d)$ experionced by each colicrence alter a pulse with phase $\phi$ is proportional to the coherence number change $(\Delta p)$ during coherence t.ransfor.

$$
\Delta \phi=-\Delta \mu \phi
$$

Thus different phase factors are experienced for different final coherences. This is the key to the solection of coherence pathway, which is introduced later.

The existence of more llan l wo cmergy levels for a quadrupolar nucleus in highfield greatly complicalus llace affece of an RP pulse on the spin system [26, 27]. In this case, the relative magnitude of $w_{Q}$ and $u^{\prime}$ deremines the exact effect of the pulse on the spin system. In onc extrome. When w $\gg w_{Q}$. the Rl pulse is a hard pulse and a muclear spin mutales just like a non-cyuadrupolar spin. On the other hand, if $\omega_{1} \ll \omega_{Q}$, the pulse is a soft pulse and only the contal transition is excited. The spin then nutales $I+\frac{1}{2}$ times as fast as a non-quadrupolar spin. In this respect, it is not unique 10 deline $90^{\circ}$ pulse lenght for a quadrupolar mucleus. When a soft pulse is used, the $90^{\circ}$ pulse longht reported is the solid-state pulse length; When a hard pulse is instoad used, lhe pulse length is the liquid-state pulse length (since in the liquid state, the aromged (quadrupolar coupling comstant is $\%(0)$, which is $I+\frac{1}{2}$ 
times as long as the solid-stat.e pulse length. The different definitions of pulse length sometimes cause confusion. We shall go back to the excitation problem in chapter 3 when multiple-quantum experiments are involved.

\subsubsection{Free Induction Decay}

Free incluction decay (FID) is the evolution of the spin system after or between RF pulses. The evolution is governed by the tolal IIamiltonian of the system, which may consist of many intcrnal spin Ilamiltonians.

$$
H_{F I D}^{G S}=H_{C S S}+H_{Q}+H_{D}
$$

The evolution of the densily opcrator is clescribed by

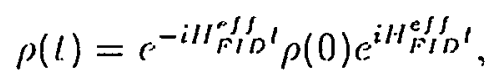

if $H_{F I D}^{e f f}$ is time-independenl.

Free induction decay' docs not cause the crealion and destroy' of coherences, thus no coherence transfer is involved. However, a phase factor is still experienced for each coherence.

$$
\begin{aligned}
& \left(l: I D I_{0}\left(I_{I: 11}^{-1}=I_{0}\right.\right. \\
& l_{F: 1 D} l_{ \pm}(:-1)=l_{ \pm} c^{\mp i \Omega I}
\end{aligned}
$$

In these equalions.

$$
l_{F / 1)}=c^{-i\|\|_{F(D)}^{E r l}}
$$

and $\Omega$ is a sum of the shift frefunencies due to chemical shift and 2nd-order quadrupolar interaction.

$$
\Omega=w^{c^{\prime} S}+w^{2 Q}
$$

Some important points are worl.1 noting here: 
I. If the spin system is in /o state, it does not avolve when there is no pulse applied. As a rosult. When a $90^{\circ}$ pulso is applied to the magnetization in the $x-y$ planc, the final density matrix has an $I_{0}$ component that does not evolve after the pulse. 'This component can be kept for a short time (tens of millisconds 10 many soconds) and restored by another $90^{\circ}$ pulse. The pair of $90^{\circ}$ pulses separated by a short delay used for storing magnelization is called a z-filter. The stored magnetization actually decays slowly during the dolay betweon the pulses. The decay is the result of exchanging (nnergy between lihe spin rescrvoir and its environment, and can be chatacterelized by an exponemtial decay with time constant $T_{1}$ (spin-latitice relaxation) [2ㅅ. 20)].

$$
.11_{0}-.1 y_{\%}=.1 /_{0} c^{-1 / T_{1}}
$$

Here $M_{Z}$ is the remaining magnetimation in the $z$-direction and $M_{0}$ is the magnetization When the sistom is al cquilibrium. z-filter is used in DAS to store magnelizalion along the z-axis during the reorientation of spinner axis.

II. The phase factor that is accummlated during [ill is dependent on the resonance frectuency S?. This is where the chemical shilt and quadrupolar

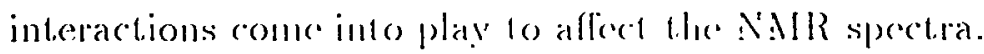

III. The shift frecuencies for hle +1 and - I cohereness have opposite signs. If the +1 and -1 coherences are both deteredel mirror inage of the real peaks is expectod. This is not a problem in a simple one-dimensional NMR experiment, as the dencelor records only the signal from $l_{+}$coherence. In a two-dimensional experiment. this does canse problems that need to be treatied carcofulle: 


\subsubsection{Example: A Simple 1D NMR Experiment}

As an example, let us consickr the evolution of the density matrix of a spin system in a simple one-dimensional NMR experiment shown in Figure 2.5. We also assume

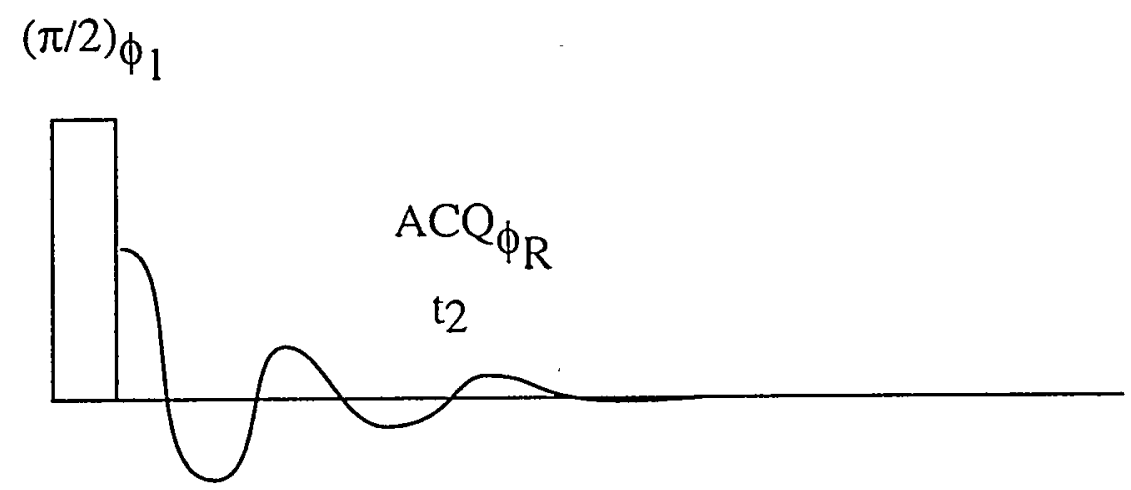

Figure 2.5: Pulse secucuce for a simple one-dimensional NMR experiment.

that all of the spins lave the same resonance frequency $\Omega$. The experiment has only one $90_{x}^{\circ}$ pulse $(\phi=0)$. which is followed by quadralure detection with $\phi_{r}=90^{\circ}$. The density matrix at equilibration is $I_{0}$, which after the pulse becomes a mixture of $I_{+}$ and $I_{-}$(Eqn 2.109).

$$
I_{0} \rightarrow \frac{i}{\sqrt{2}}\left(I_{+}+I_{-}\right)
$$

This mixed state after delay $l$ becomes (Eqn 2.117)

$$
\frac{i}{\sqrt{2}}\left(I_{+}+I_{-}\right) \rightarrow \frac{i}{\sqrt{2}} \cdot\left(I_{+} e^{-i \Omega t}+I_{-} e^{i \Omega t}\right)
$$

The signal is then expressecl ass

$$
\therefore(l)=c^{-i \pi / 2} \operatorname{Tr} r\left(\rho(l) I_{+}\right)=c \cdot r p(i \Omega \Omega l) .
$$

which after complex Fouricr transformation, gives a $\delta$-function in the frequency domain. 
The line observed in a ral NMIR experimont is nover a $\delta$-function but has a finite linewidth. Since the sample has a finite volume, different parts of the sample have slightly different resonance frequencies due to the field inhomogeneity. This frequency difference broadens the NMIR spectrum. Impurities in a sample may contribute to the broadening as well. Even if all of these factors do not exist, the spin-spin relaxation dephases the coherences and still broadens the spectra. Notice that a nuclear spin is not isolated in the systcm, its interaction with enviromment could add a random phase factor to the colicrence 'This causes the clephasing of the magnetization whose net effect can often be approximated by an exponential decay with a time constant $T_{2}$ (spin-spin relaxalion limc) [30]. Including this decay in Eqn 2.123 gives the experimentally detectod signal.

$$
S(1)=\epsilon^{-t / T_{2}, i \Omega t}
$$

Fourier transformation of this signal results in a Lorm zian-shape peak at frequency $\Omega$.

\subsubsection{Coherence Pathway}

It is common in NMIR that muliple pulses are used. Since an RF-pulse creates and mixes colierences of different orders and ach colnerence evolves with different frequencies, after man! pulsos. lor resultant donsily matrix may be very complicated. Even though the detertor only pieks up the-I coherence, the signal still comes from spins followed different patls and is quite complex. The colnenence orders that the

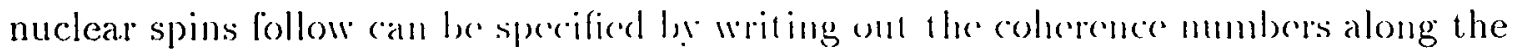
path sequentially (colerence pathway). For example, in Figure 2.6, the colierence pathway designated by the bolded lino is the $0 \rightarrow-1 \rightarrow 0 \rightarrow-1$ pathway, whereas the dotted line is lihe $0 \rightarrow+1 \rightarrow 0 \rightarrow-1$ pathway:

In a multiple-pulse N.MlR experiment the desired signal often eomes from a specific 

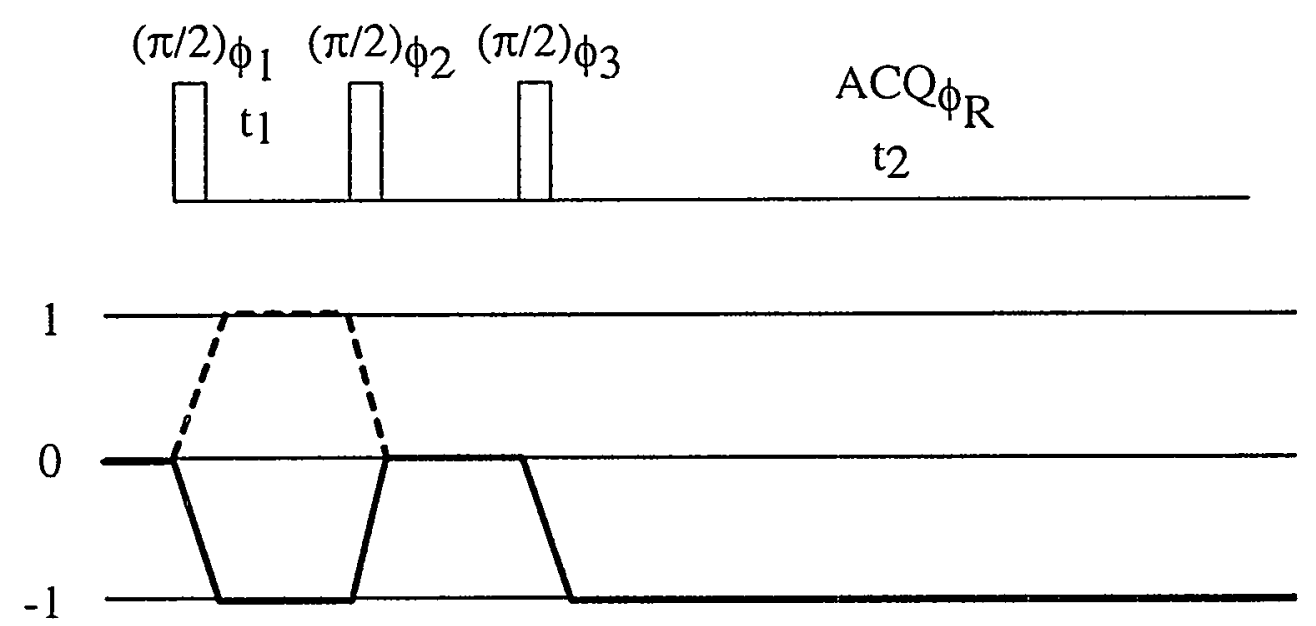

Figure 2.6: The colerence pallhway for a simple DAS experiment. The bolded and dashed lines are the desired signal.

coherence pathway. To get, ricl of the signal from other pathways, we can add up signal from many different experiments in which the phases of the pulses and the receivers are systematically incremented (phase cycle). Some empirical rules that are used to design correct phase cycles are listed below $[31,32,33]$.

I. The last pulse needs no plase cycle since the detection ensures that the -1 coherence is selectecd.

II. If a pulse is changed in phase by $\phi$, then a coherence undergoing a change in coherence order of $\Delta p$ experiences a phase shift of $-\Delta p \cdot \phi$.

III. If the coherence order changes along a desired coherence palluway is $\left(\Delta p_{1}, \Delta p_{2}, \cdots p_{n}\right)$. l.he receiver phase should be set to

$$
o_{r}=-\sum_{i=1}^{n} \Delta p_{i} \cdot \phi_{i}
$$

to ensure the sclection of this pathway. Here, $\phi_{i}$ is the phase of the $i$ th RF pulse. 
IV. To restrict the colsenence tansfer undor a pulse to a particular change $\Delta p$ in coherence order, we my perform $m(m>|\Delta p|)$ experiments with the RF phase

$$
\phi_{i}=2 k_{i} \pi / m
$$

where $k_{i}=0,1, \cdots, m-1$.

V. The above procedure retains not only the coherence undergoes a change $\Delta p$, but also those with changes equal to $\Delta p \pm n m$, where $n$ is an integer.

As an example, consider l.he colorence pathway of a simple DAS experiment (Figure 2.6). Here, both the bolded and the lashed pathways are to be retained. The first pulse induces colerence tansfor with $\Delta p= \pm 1$. According to rule IV, this pulse need to be cycled through at lasis two phases $\left(0^{\circ}, 180^{\circ}\right)$. According to rule $V$, the phase cycle relains all the odd-order cohorences, and rejects the even-order coherences. Similarly. the second pulse is cycled through two phases $\left(0^{\circ}, 180^{\circ}\right)$ to guarantee coherence lansler with $\Delta p= \pm 1$. Tho third pulse can be left uncycled since receiver picks up the - I coherence order. IIsing Equ 2.125, the receiver phases can be determined and the following A-step phase cycle is obtained.

$$
\begin{aligned}
& \dot{\sigma}_{1}=0^{\circ} \cdot 0^{\circ} \cdot 180^{\circ} \cdot 180^{\circ} \\
& \dot{\sigma}_{2}=0^{\circ} \cdot 180^{\circ} \cdot 0^{\circ} \cdot 180^{\circ} \\
& \dot{c}_{3}=0^{\circ} \cdot 0^{\circ} \cdot 0^{\circ} \cdot 0^{\circ} \\
& \dot{c}_{r}=0^{\circ} \cdot 180^{\circ} \cdot 180^{\circ} \cdot 0^{\circ}
\end{aligned}
$$

In an experiment that involves a lot of pulses. it may be possible to leave some pulses uncycled. In the above D. DS experiment. the delay betwern the second and

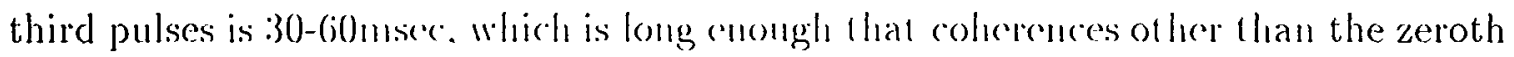
order (the $I_{0}$ state) disappear due to relaxalion. 'This means that the long delay kills 
all the unwanted coherences and phase cycle of the sccond pulse is unnecessary. The simplified two-step phase cycle is given below.

$$
\begin{aligned}
& \dot{\phi}_{1}=0^{\circ}, 180^{\circ} \\
& \dot{\phi}_{2}=0^{\circ}, 0^{\circ} \\
& \dot{\phi}_{3}=0^{\circ}, 0^{\circ} \\
& \dot{\phi}_{r}=0^{\circ}, 180^{\circ}
\end{aligned}
$$

More examples of how to construct correct phase cycles are discussed in chapter 3.

\subsection{Two-Dimensional NMR}

\subsubsection{Basics}

A two-dimensional NMR exporiment has two distinct, free evolution periods, usually separated by RF pulses to enhance the information content of the spectra. The basic scheme of the experiment. is shown in Figure 2.7 and four different intervals exist in the experiment. During the preparation period, the spin system is prepared in a coherent, state froun the cepuilibrat.ed statc $I_{0}$ l.hrough RF pulses. In the course of evolution period. the spin system is allowed to undergo free evolution under the effective Hamiltonian $/{ }_{F}^{(1)} \| d$. The superscipt designates that this Hamiltonian is the Hamiltonian during the first crolution period. The evolution period is made variable in a two-dimensional experiment, allowing the sampling of $1 /$-evolution. The mixing period may consist of one or more pulses, sepalmalced by constant intervals. For example, in the DAS rexpriment shown in Figure 2.6, the sceond and third pulses separated by a time inceral (z-filter) comprises the mixing period. The mixing process introduces perturbation to the spin system so that the effective Hamiltonian after mixing period $\left(I_{F I D}^{(2)}\right)$ is oficn different from $H_{F I D}^{(1)}$. The detection period is similar to that in a onc-dimensional experimont. 


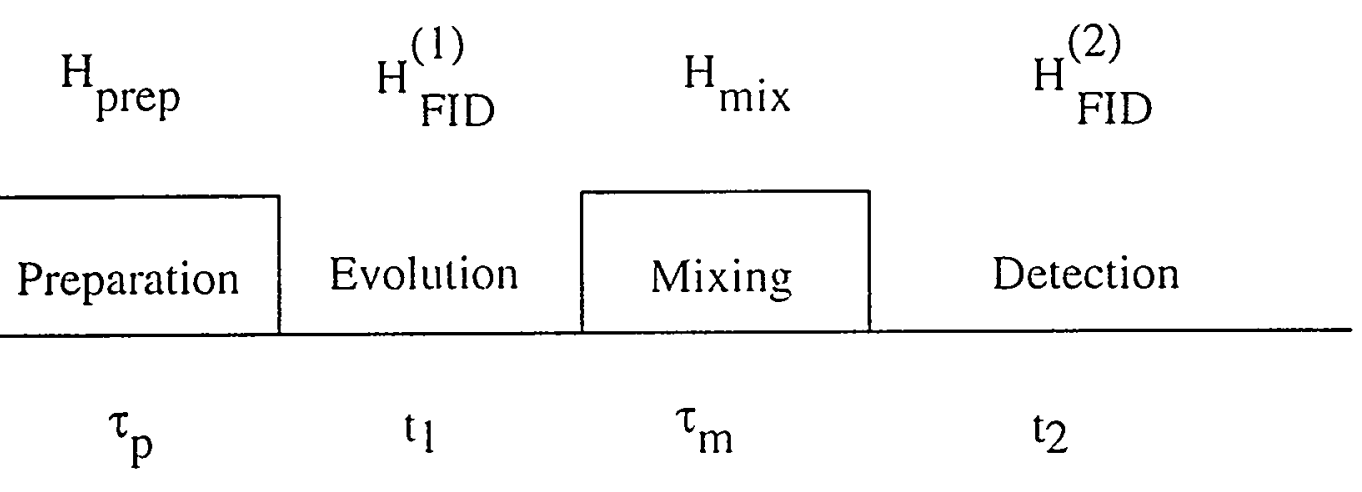

Figure 2.7: A schematic diagram of a two-dimensional NMR experiment.

Assuming that the fiecuency shifts in the evolution period and the detection period are $\Omega_{1}$ and $\Omega_{2}$ respectively. the signal from a two dimensional experiment can often be described by the product, of two free induction decay signals.

$$
S\left(t_{1}, l_{2}\right)=e^{-t_{1} / T_{2}} c^{i \Omega_{1} t} c^{-t_{2} / T_{2}} e^{i \Omega_{2} t}
$$

After Lwo-dimensional Fourice 1 ransformation. Frefuency domain spectrum is obtained which reflects the corrolation betwern the two frecuency domains.

\subsubsection{Pure-Absorption Phase 2-D NMR Lineshape}

Fourier t.ransformalion of E(1n 2.12 . 9 actually docs not give a spectrum with best resolution. The frequency spectrum cont ains negative inteensity (dispersive) and does not have pure plase. To see the problem clearly consider the Fourier transformation of the $l_{2}$ dimansion of l:(1n 2.129 .

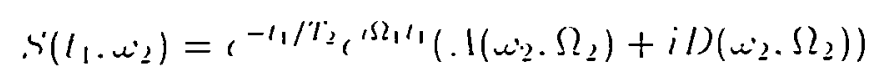

Here $A(\omega, \Omega)$ and $D(\omega, \Omega)$ are the alsorplive and dispersive lineshape functions with a peak at $\Omega$ in the $\omega$ dimcusion.

$$
A(\omega \cdot \Omega)=\frac{T_{2}}{1+\left(\omega-\Omega()^{2} T_{2}^{2}\right.}
$$




$$
D(\omega, \Omega)=\frac{(\omega-\Omega)^{\prime} \Gamma_{2}^{\prime 2}}{1+(\omega-\Omega)^{2} T_{2}^{2}}
$$

After a second Fourier transformation, the signal in the frequency domain is then

$$
\begin{aligned}
S\left(\omega_{1}, \omega_{2}\right)= & \left(A\left(\omega_{1}, \Omega_{1}\right)+i D\left(\omega_{1}, \Omega_{1}\right)\right)\left(A\left(\omega_{2}, \Omega_{2}\right)+i D\left(\omega_{2}, \Omega_{2}\right)\right) \\
= & A\left(\omega_{1}, \Omega_{1}\right) A\left(\omega_{2}, \Omega_{2}\right)-D\left(\omega_{1}, \Omega_{1}\right) D\left(\omega_{2}, \Omega_{2}\right) \\
& +i\left(A\left(\omega_{1}, \Omega_{1}\right) D\left(\omega_{2}, \Omega_{2}\right)+D\left(\omega_{1}, \Omega_{1}\right) A\left(\omega_{2}, \Omega_{2}\right)\right)
\end{aligned}
$$

The imaginary component can be dropped now and the real component is displayed. The real component contains the $-D\left(\omega_{1}, \Omega_{1}\right) D\left(\omega_{2}, \Omega_{2}\right)$ term which leads to a phasetwisted lineshape. The desired signal is $A\left(\omega_{1}, \Omega_{1}\right) \cdot A\left(\omega_{2}, \Omega_{2}\right)$ only.

One of the solutions (States method) [34] is to acquire the data in a hypercomplex fashion. Instead of recording a single datiaset. two datiasets $S_{c}\left(t_{1}, t_{2}\right)$ and $S_{s}\left(t_{1}, t_{2}\right)$ are recorded separately. Mathomalically, the two signals correspond to the cosine and sine portions of the signal in the $l_{1}$ dimension.

$$
\begin{aligned}
& S_{c}\left(l_{1}, l_{2}\right)=\cos \left(\Omega_{1} l_{1}\right) e^{-\left(t_{1}+l_{2}\right) / T_{2}} e^{i \Omega_{2} t_{2}} \\
& S_{s}\left(l_{1}, l_{2}\right)=\sin \left(\Omega_{1} l_{1}\right) e^{-\left(f_{1}+t_{2}\right) / T_{2}} e^{i \Omega_{2} l_{2}}
\end{aligned}
$$

Fourier transformation about. $l_{2}$ is then scparately done on both datasets.

$$
\begin{aligned}
& S_{c}\left(l_{1}, \omega_{2}\right)=\cos \left(\Omega_{1} / 1\right) e^{-t_{1} / T_{2}}\left(A\left(\omega_{2}, \Omega_{2}\right)+i D\left(\omega_{2}, \Omega_{2}\right)\right) \\
& S_{s}\left(t_{1}, \omega_{2}\right)=\sin \left(\Omega_{1} / 1\right) e^{-t_{1} / T_{2}}\left(A\left(\omega_{2}, \Omega_{2}\right)+i D\left(\omega_{2}, \Omega_{2}\right)\right)
\end{aligned}
$$

The imaginary compononts of both datasets are then dropped and the real components are combincel approprialdy to form a clatased

$$
\left.S\left(l_{1}-\omega_{2}\right)=A\left(\omega_{2}\right) \Omega_{2}\right) c^{-l_{1} / T_{2}} c^{-i \Omega_{1} t_{1}}
$$

The Fourier transformation of this dataset with respect to $l_{1}$ is

$$
S\left(\omega_{1}, \omega_{2}\right)=A\left(\omega_{2} . \Omega_{2}\right)\left(A\left(\omega_{1}, \Omega_{1}\right)+i D\left(\omega_{1}, \Omega_{1}\right)\right) \text {. }
$$


Only the real channol of this signal is showed and llar spectrum is of pure-absorption phase lineshape.

In the simple DAS experiment shown in Figure 2.6, the bolded line gives the signal in Eqn 2.129. In order to get. $S_{c}$ and $S_{s}$, it is essential to retain the coherence pathway designated by the dashed line. The signal corresponding to the dashed line is

$$
S^{\prime \prime}\left(l_{1}, I_{2}\right)=e^{-t_{1} / T_{2}} e^{-t \Omega_{1} t} e^{-t_{2} / T_{2}} e^{i \Omega_{2} t}
$$

The detected signal is thon

$$
S_{c}\left(l_{1}, l_{2}\right)=S+S^{\prime \prime}=2 \cos \left(\Omega_{1} l_{1}\right) c^{-l_{1} / T_{2}} e^{-l_{2} / T_{2}} c^{1 \Omega_{2} t_{2}}
$$

For the last ecpuality:

$$
\cos (\Omega) 1)=\frac{c^{-\cos 21}+c^{1 s 2 t}}{2}
$$

is used.

The sine part of the signal can be obtained by phase shifting the first pulse by $-90^{\circ}$, and keeping the phases of all other pulses unclianged. The detected signal is thus

$$
\begin{aligned}
& S_{s}\left(l_{1}, l_{2}\right)=c^{-1 \pi / 2}, 5+c^{2 \pi / 2}, 5^{\prime \prime} \\
& =2 \sin \left(\Omega \Omega_{1} l_{1}\right) c^{-t_{1} / T_{2}}\left(-t_{2} / T_{2} \epsilon^{-1 S \Omega_{2} t_{2}} .\right.
\end{aligned}
$$




\section{Chapter 3}

\section{High-Resolution NMR of Quadrupoles}

In the preceding chapter, the orientational dependence of the chemical shift and quadrupolar interactions were derived. Conventional sample spinning techniques (magic-angle spinning (MAS') and variable-angle spinning (VAS)) are first introduced in this chapter and are shown to be ineffective to average out the 2nd-order quadrupolar interaction. Dynamic-angle spinning (DAS) and multiple-quantum magic-angle spinning (MQMAS') methods are then presented which reconstruct high-resolution isotropic spectra for quadrupolar nuclei. Experimental results with both techniques are shown and compared in detail.

\subsection{Magic-Angle and Variable-Angle Spinning}

Magic-angle spinning ( MAS) was first developed by Andrew [35, 36] to remove heteronuclear dipolar compling and chemical șhift anisotropy that, a spin- $\frac{1}{2}$ nucleus experiences. Combined with cross polarization (CP) [13], it allows routine rapid collection of proton-cnhanced ${ }^{13}$ (' spectira with liquid-like resolution in most solidstate NMR laboratorics [1-1]. 'The principles of the MAS' technique were discussed in chapter 2, where the 1st-order frecuucucy shift due to chemical shift was written as

$$
\omega^{c s s}\left(a^{c s} \cdot 3^{c s}\right)=\omega_{i s s}+A_{2}^{c s}\left(\alpha^{c s} \cdot \beta^{c s}\right) P_{2}(\cos \theta)
$$

The symbols all have thoir common moanings. It is instantaneously clear that spinning the sample at the magir-angle ( $M A S .0)=54.74^{\circ}$ ) averages out the orientationdependent terms in the above copuation $\left(P_{2}(\cos \theta)=0\right)$.

Figure 3.1 is the simulated static and MIAS spect ra of a spin- $\frac{1}{2}$ nucleus at two 

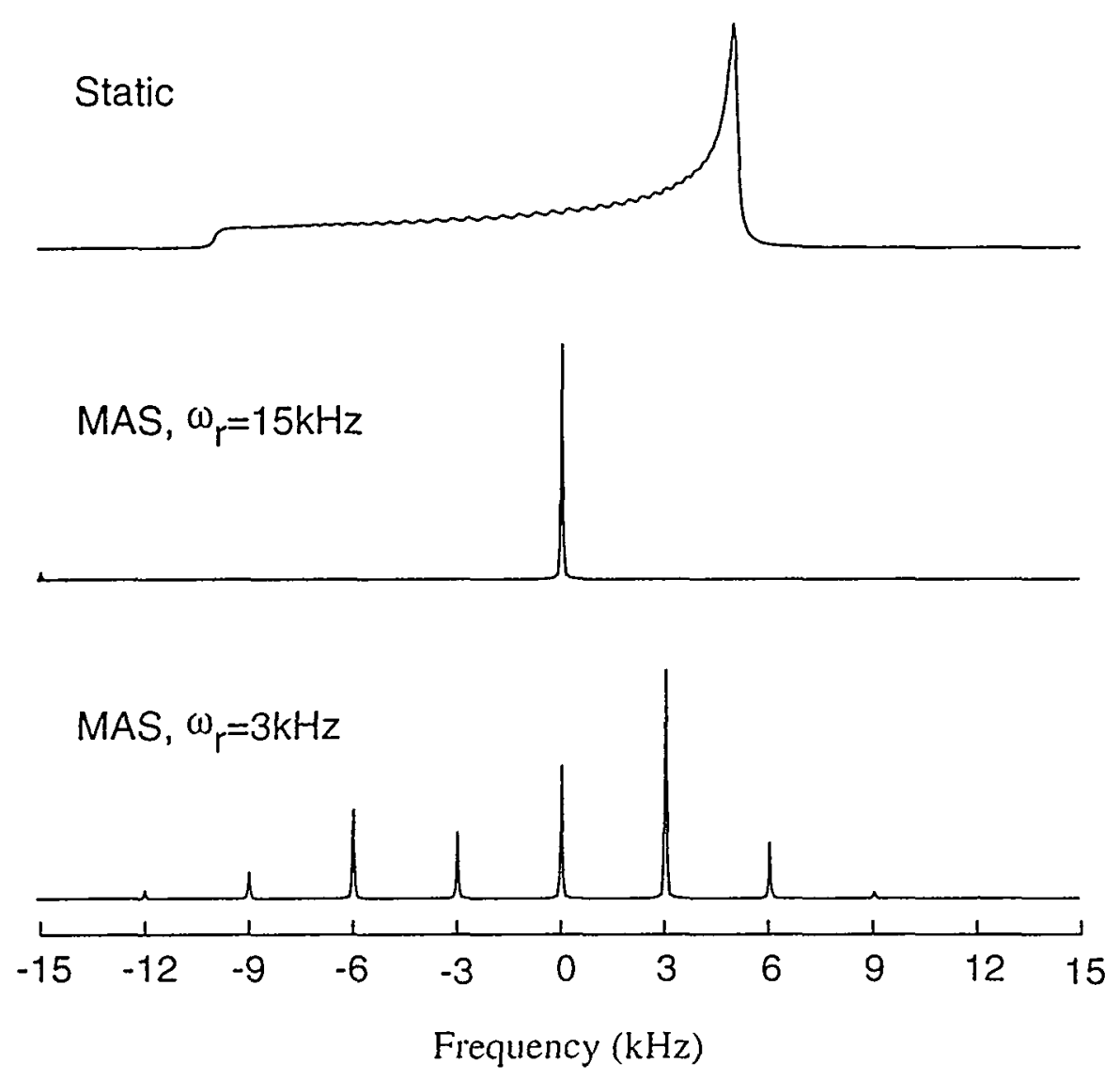

Figure 3.1: Simulated static and MAS spectra of a spin- $\frac{1}{2}$ nucleus. The parameters used for simulation are $\delta_{i s o}=0.0 p p m . \delta_{c s}=100 p p m, \eta_{c s}=0.0, \omega_{0}=100 \mathrm{MHz}$.

spinning rates. As expected. MAS removes the anisotropy of chemical shift interaction, leading to a sharp poak at the isotropic chemical shift position. It is also clear that additional lines may appear at $\delta_{i s o}+n \omega_{r}$ when the spinning speed $\omega_{r}$. low. These lines are spinning sirlebands that come from the modulation of the free evolution by the sample rotation. The morlulation originates from the time-dependent terms wo negleced in lipus 2. (ix and 2.76. and will not be considered in detail in this

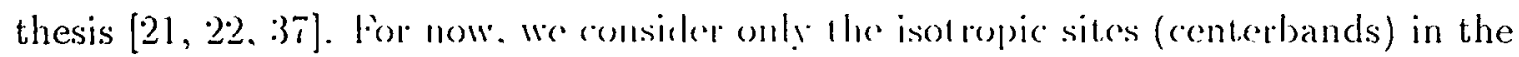
spectra. Experimentally, discriminaling conterbands from sidebands is overcome by performing measurements at lwo spinning rates and the peaks that do not shift will be the isotropic sites.

MAS is also cflicion in removing the Ist-order quadrupolar interaction. Again, sidebands 
are expected [19].

$$
\omega_{m \leftrightarrow m-1}^{1 Q}=\frac{3(2 m-1) \hbar C_{Q}}{4 I(2 I-1)} P_{2}(\cos 0)\left(\frac{3 \cos ^{2} \beta^{Q}-1}{2}+\frac{\eta_{Q}}{2} \sin ^{2} \beta^{Q} \cos 2 \alpha^{Q}\right)
$$

The difficulty is, however, that the 1st-order quadrupolar interaction is often so large that the satellite transitions ( $m \leftrightarrow m-1$ transitions,$m \neq \frac{1}{2}$ ) that are broadened by the 1st-order interaction are not observable (see $[17,18,19]$ ) for opposite examples). The central transition is further broadened by end-order quadrupolar interaction, which has scalar and $P_{2^{-}}, P_{1^{-}}$-dependent terms thati give the frequency expression below (see also, Eqn 2.\$1).

$$
\omega^{2 Q}\left(\alpha^{Q}, \beta^{Q}\right)=\omega_{i s o}^{2 Q}+A_{2}^{Q}\left(\alpha^{Q}, \beta^{Q}\right) P_{2}(\cos \theta)+A_{4}^{Q}\left(\alpha^{Q}, \beta^{Q}\right) P_{4}(\cos \theta)
$$

The dependences of $P_{2}(\cos \theta)$ and $P_{4}(\cos \theta)$ on the spinning angle $\theta$ is shown in Figure 3.2. It is obvious l.hat $P_{2}(\cos \theta)=0$ aind $P_{4}(\cos \theta)=0$ have no common roots. This means that spinning the sample at the magic-angle (MAS) or at an angle other than $54.74^{\circ}$ (variable-angle spinning, VAS) is not effective for the removal of the 2nd-order quadrupolar anisot.ropvi. As an example, Figure 3.3 shows the ${ }^{23} \mathrm{Na}$. $9.4 \mathrm{~T}$ static and MAS spectira of $\mathrm{Na}_{2} \mathrm{C}_{2} \mathrm{O}_{1}$. The MAS spectrum is narrower than the static spectrum by a factor of 5 , but. is still broadened to $4 \mathrm{kH} / \mathrm{Hz}$ by the residue 2nd-order quadrupolar interaction.

To further clemonstratic the effect of sample spinning on the quadrupolar lineshapes, Figure 3.4 shows the simulated spect wa with diflerent quadrupolar asymmetric parameters $\left(\eta_{Q}\right)$ and spinning angles $(0)[38.39]$. I1 is interesting that, when $\eta_{Q} \approx 0$, spinning the sample at. $79.19^{\circ}$ or $37.38^{\circ}$ gives betuer resolution than MAS $[40,41]$. Nevertheless, this is not, true when $\eta_{0}$ approaches unity, and the spectra are rarely as sharp as the MAS spectar of spin- $\frac{1}{2}$ mucloi. Dymamic-angle spinning (DAS) and multiple-quantum magir-angle spimning ( MQMAS) are two of the solutions that over- 


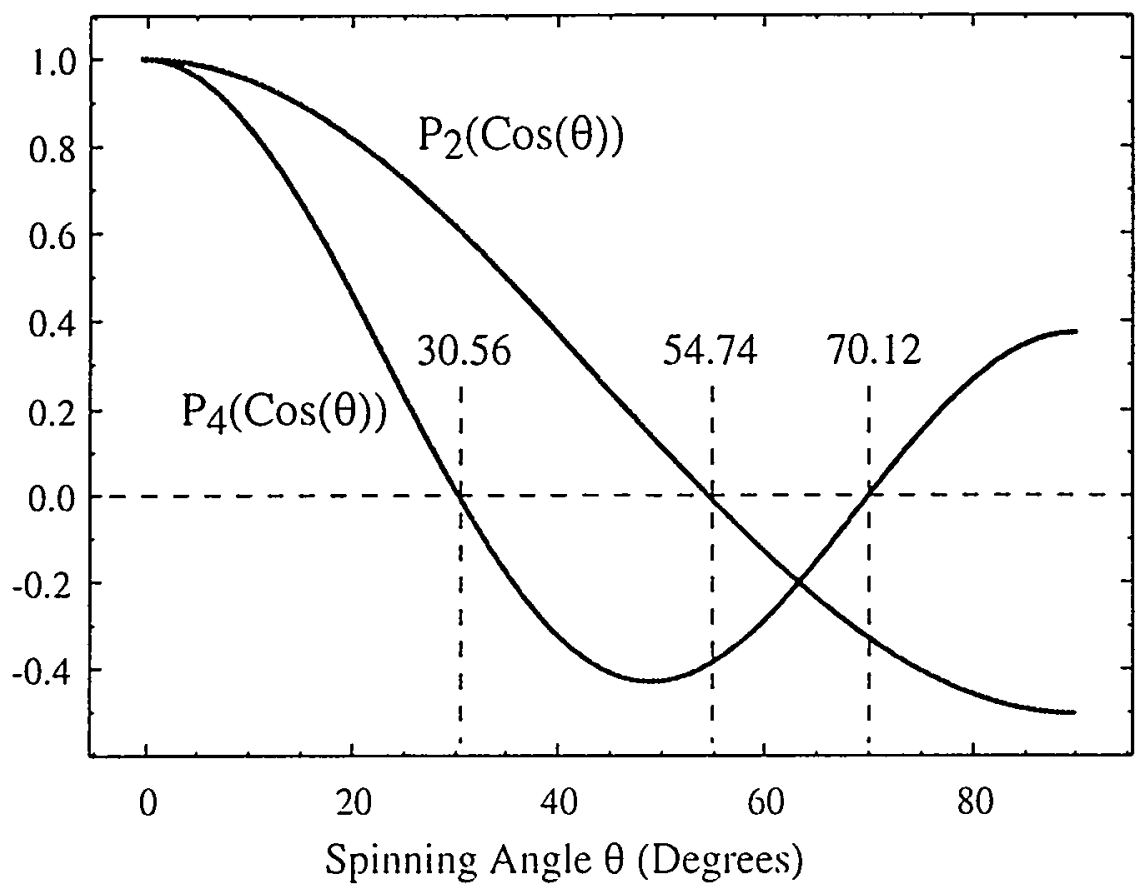

Figure 3.2: 2nd and $41 \mathrm{~h}$ order legendre polynomials as a function of spinner angle. There is no single angle at, which both polynomials are zero.
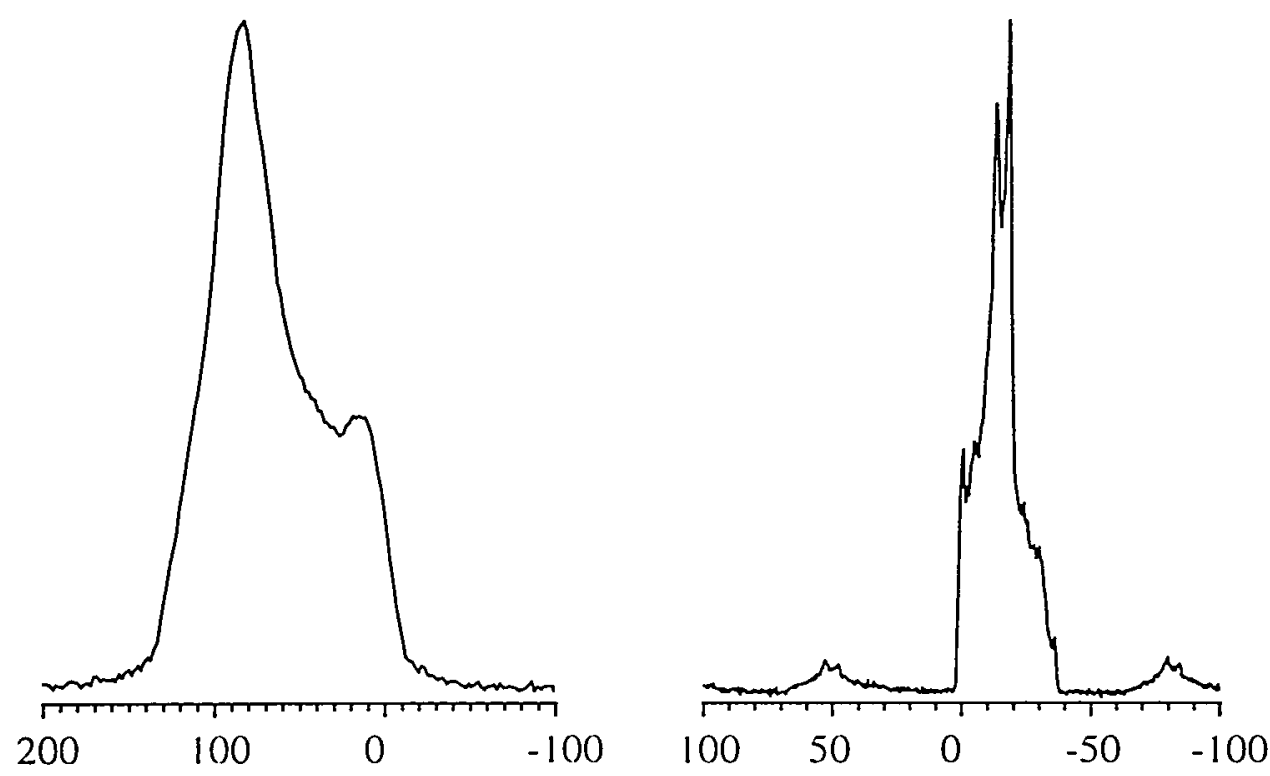

Fiequency (ppm firom IM NaCl)

Figure 3.3: ${ }^{23} \mathrm{Na}$ statice and MISS spect.ra of $\mathrm{Na}_{2}\left(\mathrm{O}_{2} \mathrm{O}_{1}\right.$ at 9.4T. The MAS spectrum is narrower than the static sperefrum. Inu is still broadened to about 1 kilohertz. 
come this resolution problem and will be discussed primarily in this chapter. Alternative solutions such as clouble rotation (DOR) and dynamic-angle hopping (DAH) are also briefly discussed at the end of this chapter.

\subsection{2nd-order Averaging: Theory}

The analysis in section 3.1 reveals a major problem to overcome in the NMR of half-integer quadrupolar spins. The central transition, which are not broadened to the 1st-order by quadrupolar. inloraction, remains broad under MAS or VAS since the frequency expression for 2 nd-order quadrupolar interaction contains two anisotropic terms that depend on $P_{2}(\cos \theta)$ and $P_{1}(\cos \theta)$ respectively $(\operatorname{Egn} 2.83)$.

$\omega_{m \leftrightarrow-m}^{2 Q}=C_{0}(I, m) \omega_{i s o}^{2 Q}+C_{1}(I, m) A_{2}^{Q}\left(\alpha^{Q}, \beta^{Q}\right) P_{2}(\cos \theta)+C_{2}(I, m) A_{4}^{Q}\left(\alpha^{Q}, \beta^{Q}\right) P_{4}(\cos \theta)$

\subsubsection{Halm-Echo Experiment}

The simplest experiment that removes the 2nd-order quadrupolar broadening, is the Hahn-echo experiment. The experiment consists of a $90^{\circ}\left(\phi_{1}\right)$ pulse followed by a delay $t_{1} / 2$ followed by an $180^{\circ}\left(\phi_{2}\right)$ pulse followed by another delay $t_{1} / 2$ and then acquisition with recriver phase $o_{2}$ (Figure 3.5). When $t_{1}$ is incremented in a two-dimensional fashion. 1.his experiment allows the measurement of the intrinsic $T_{2}$ relaxation time.

The observed signal in this experiment may be calculated as follow. The initial density matrix $I_{0}$ evolves during fhe first pulse into a mixture of two states.

$$
I_{0} \stackrel{s 0_{i g}^{\infty}}{\longrightarrow} \frac{i}{\sqrt{2}}\left(I_{+} e^{-i \phi_{1}}+I_{-} e^{i \omega_{1}}\right)
$$




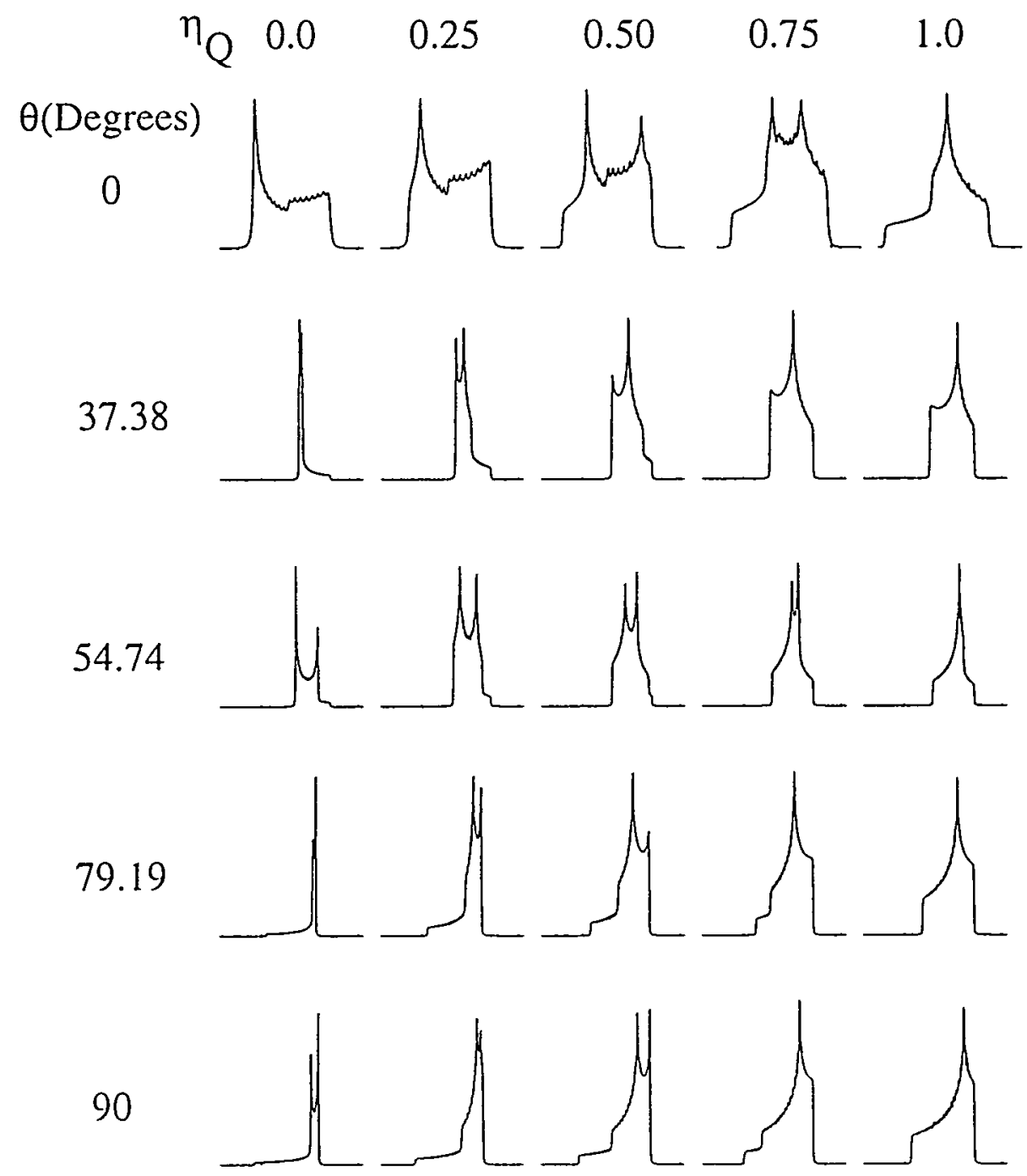

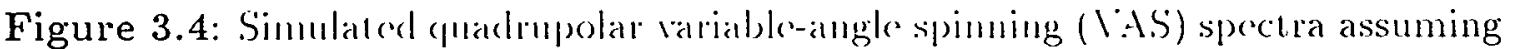
infinite spinning rate. The rerlical sale is not lhe same for different spectra. 

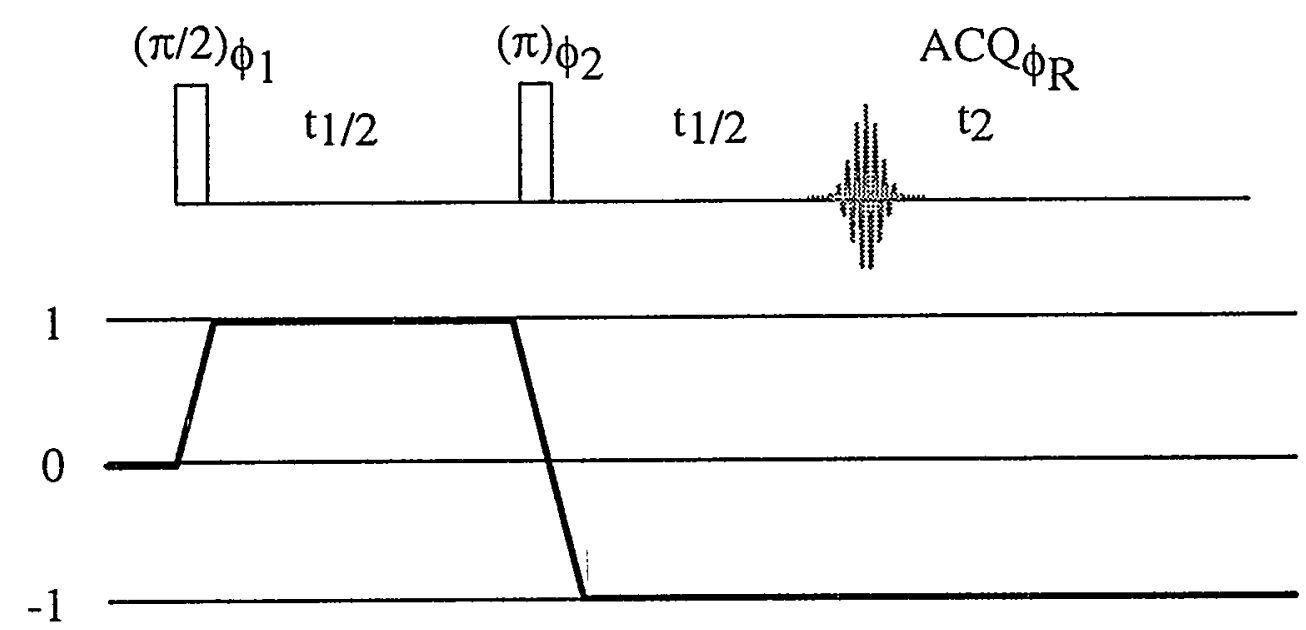

Figure 3.5: $90^{\circ}-180^{\circ}$ IIahn-ccho sequence and coherence pathway. This sequence allows the measurement of intrinsic spin-spin relaxation time $T_{2}$. The sequence refocuses the 2nd-order quadrupolar interaction, together with the isotropic chemical shift and 2nd-order quadrupolar shift.

The states continue to evolve under the effective FID Hamiltonian for a time $t_{1} / 2$.

$$
\frac{i}{\sqrt{2}}\left(I_{+} e^{-i \phi_{1}}+I_{-} e^{i \phi_{1}}\right) \stackrel{F l D}{\longrightarrow} \frac{i}{\sqrt{2}}\left(I_{+} e^{-i\left(\phi_{1}+\Omega t_{1} / 2\right)}+I_{-} e^{i\left(\phi_{1}+\Omega t_{1} / 2\right)}\right)
$$

When the $180^{\circ}$ pulse is applied, the system evolves into

$$
\begin{aligned}
\frac{i}{\sqrt{2}}\left(I_{+} e^{-i\left(\dot{p}_{1}+\Omega t_{1} / 2\right)}+I_{-} e^{i\left(\phi_{1}+\Omega t_{1} / 2\right.}\right) \stackrel{180_{\xi_{2}}^{\circ}}{\longrightarrow} \\
-\frac{i}{\sqrt{2}}\left(I_{-} e^{-i\left(\phi_{1}-2\left(\phi_{2}+\Omega t_{1} / 2\right)\right.}+I_{+} e^{i\left(\phi_{1}-2 \phi_{2}+\Omega t_{1} / 2\right)}\right),
\end{aligned}
$$

which then evolves for a lime $1, / 2$ before final detection is done. Since evolution does not result in any coherence transfer. the only coherence we need to consider here is the -1 coherence.

$$
\begin{aligned}
& -\frac{i}{\sqrt{2}} I_{-} e^{-i\left(\omega_{1}-2 t_{1}+\Omega t_{1} / 2\right)} \stackrel{F I l)}{\longrightarrow}-\frac{i}{\sqrt{2}} I_{-} c^{-i\left(r_{1}-2 \phi_{2}+S t_{1} / 2-\Omega t_{1} / 2\right)} \\
& S\left(t_{1}, t_{2}\right)=-\frac{i}{\sqrt{2}} e^{-\left(t_{1}+t_{2}\right) / T_{2}} C^{-i\left(\phi_{1}-2 \phi_{2}+\phi_{r}\right)} e^{i \Omega t_{2}} \operatorname{Tr}\left[I_{-} I_{+}\right]
\end{aligned}
$$




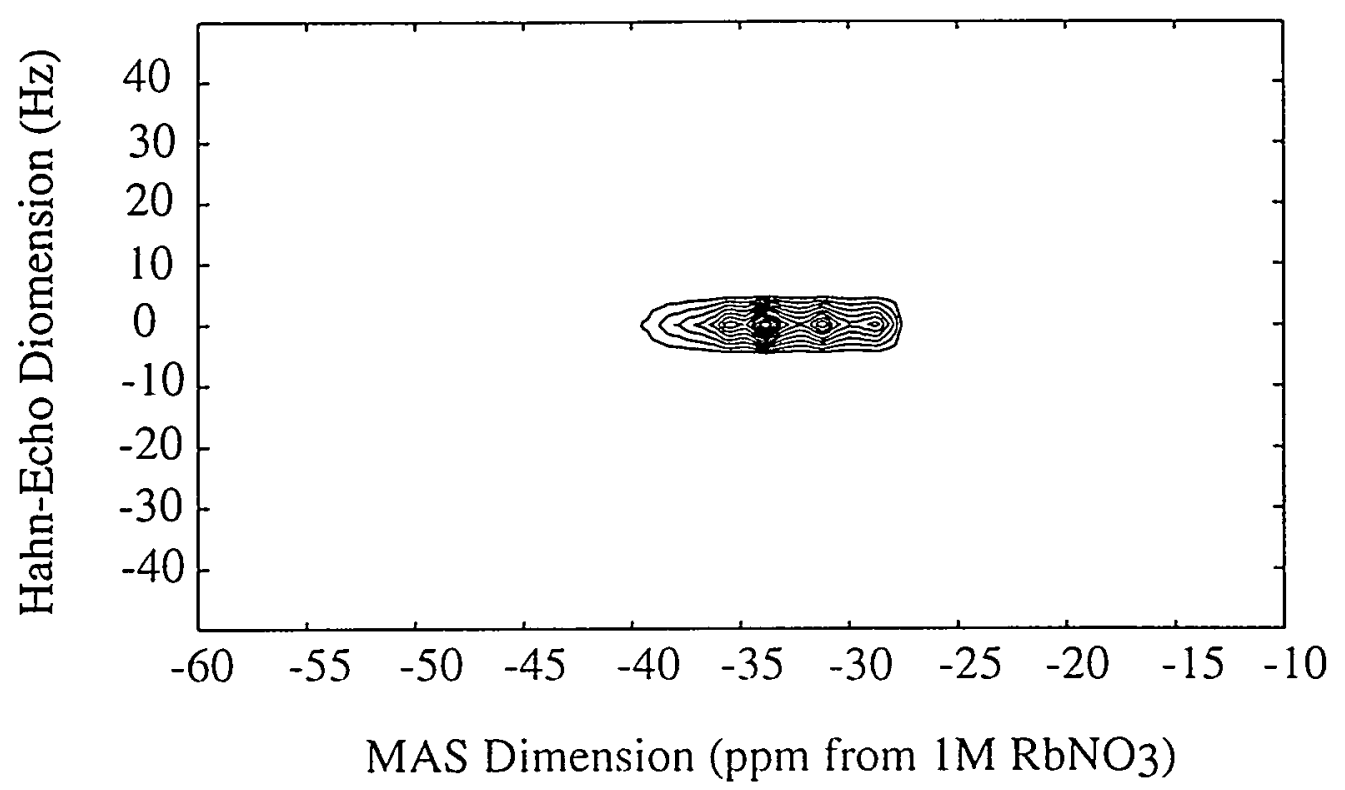

Figure 3.6: Hahn-ccho spectrum of ${ }^{8 \pi} \mathrm{Rb}$ in $\mathrm{RbNO}_{3}$ at $11.7 \mathrm{~T}$. Notice that only one zero-frequency peak is olsserved eren though there are three distinct rubidium sites in the salt.

$$
=-\frac{i}{2 \sqrt{2}} e^{-\left(t_{1}+t_{2}\right) / T_{2}} e^{-l\left(\phi_{1}-2 \phi_{2}+\phi_{r}\right)} e^{i \Omega t_{2}}
$$

Except for the $T_{2}$ decay, this signal has no $l_{1}$ dependence. The dephasing due to the chemical shift and quadrupolar interactions in the $t_{1}$ dimension is refocused, and an echo forms at the start of the accuisition. An isotropic peak is expected in the $t_{1}$ dimension (Figure 3.6) as all of the anisolropies are removed.

This method is in fact, not stutialble for practical applications since it does not discriminate chemical sites with different isotropic shifts or quadrupolar coupling constants. The $180^{\circ}$ pulse refocuses the evolution under isotropic shifts and a single zero-frequency peak is obscrecel in the isotropic dimension (Fig 3.6). The desired technique would in principle, refocus the dephasing due to the anisotropic terms in Eqn 3.3, but would not. refocus the evolution under the isotropie shifts. Both DAS and MQMAS achieve this goal. DAS accomplishes this selective averaging spatially, by spinning the sample at wo angles sorfuculially: MQMAS, instead, combines sample 
spinning and multiple-cuantum transitions to achieve this purpose. In DAS, the isotropic evolution is not distumbed so the observed shifts in the isotropic dimension are the same as those observed in DOR or DAH; MQMAS, however, partially refocuses the isotropic evolution so the observed shifts, only after correct transformation, give the DAS shifts. In this respect, both DAS and MQMAS are special types of echo experiments.

Two points are worth noting here about the echo experiment. First, the above derivation assumes that, the $90^{\circ}$ and $180^{\circ}$ pulses are ideal so that no signal from other coherence pathways is observed. Experimentally, phase cycle must be exploited to ensure that the correct pathway $(0 \rightarrow+1 \rightarrow-1)$ is selected. A four-step phase cycle is given below.

$$
\begin{aligned}
& \phi_{1}=0^{\circ}, 0^{\circ}, 0^{\circ}, 0^{\circ} \\
& \phi_{2}=0^{\circ}, 90^{\circ}, 180^{\circ}, 270^{\circ} \\
& \phi_{r}=0^{\circ}, 180^{\circ}, 0^{\circ}, 180^{\circ}
\end{aligned}
$$

Second, the $t_{1}$ dimension in a lwo-dimensional experiment can be broken up into many intervals. This gives us more frectom to prepare the spin system to achieve desired averaging. In fact. the /, dimensions of DAS and MQMAS are both broken up into two separated time intervals.

\subsubsection{DAS}

Figure 3.7 shows the DAS cxpcriment. the pulse sequence and the corresponding coherence palhway [1]. In this experimenl. the sample undergoes free precession after a $90^{\circ}$ pulse with the sample spinning at a first angle $0_{1}$ for $\frac{t_{1}}{1+k}$. Here, $l i$ is a constant that is defined later. A second $90^{\circ}$ pulse stores half of the magnetization along the $z$-axis, after which the spinner axis is flipped to $0_{2}$. A third $90^{\circ}$ pulse then brings the 

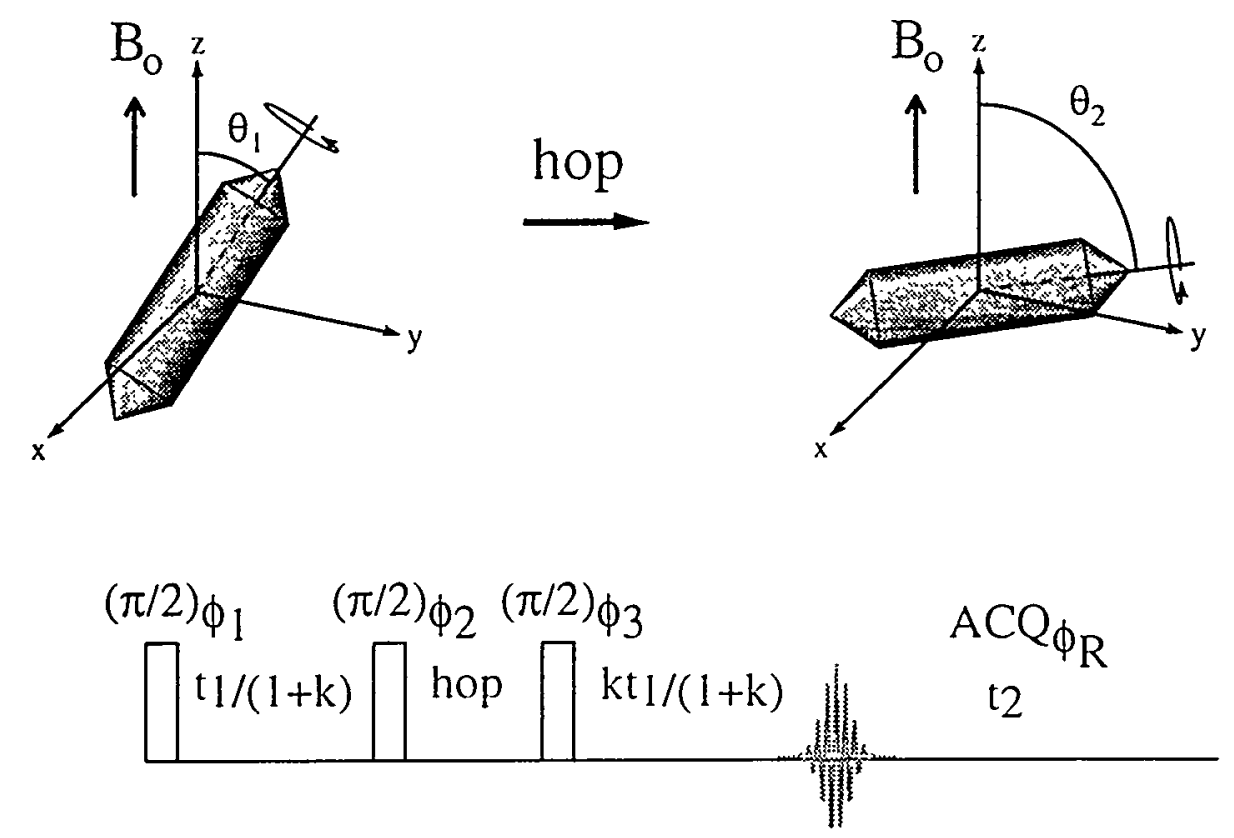

\section{1}

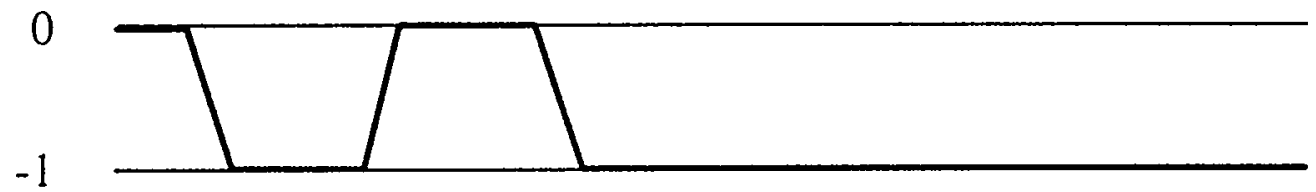

Figure 3.7: DAS experiment and pulse sequence. In this experiment, the value $t_{1}$ is incremented in a two-dimonsional fashion. During the lime interval between the $z$-filter pulses, the spinning angle is flipped from $0_{1} 1.00_{2}$. The $t_{1}$ dimension gives the isotropic DAS spectrum.

stored magnetization to the tansirerse planc ('This pair of $90^{\circ}$ pulses separated by a time interval is termed as a z-filter. It behaves as if the density matrix wore the same before and after the z-filtor. This is 1 ruc when appropriate phase cycle is performed). A DAS-echo is formed at $\frac{k \cdot t_{1}}{1+k}$ aflor the third pulse and accpuisition starts exactly on the echo top. By incromconting /, in a 1 wo-dinomsional fashion, a two-dimensional dataset is acquired which, after Fomber transform, has an isotropic $l_{1}$ dimension.

To see how the I) AS-cedo is formed, consider the End-order cuadrupolar frequency 
expressions at two angles.

$$
\begin{aligned}
& \omega_{1}^{2 Q}\left(\alpha^{Q}, \beta^{Q}\right)=\omega_{i s o}^{2 Q}+A_{2}^{Q}\left(\alpha^{Q}, \beta^{Q}\right) P_{2}\left(\cos \theta_{1}\right)+A_{4}^{Q}\left(\alpha^{Q}, \beta^{Q}\right) P_{4}\left(\cos \theta_{1}\right) \\
& \omega_{2}^{2 Q}\left(\alpha^{Q}, \beta^{Q}\right)=\omega_{i s o}^{2 Q}+A_{2}^{Q}\left(\alpha^{Q}, \beta^{Q}\right) P_{2}\left(\cos 0_{2}\right)+A_{4}^{Q}\left(\alpha^{Q}, \beta^{Q}\right) P_{4}\left(\cos \theta_{2}\right)
\end{aligned}
$$

Unlike the Hahn-echo experiment, in both parts of the echo time, the -1 coherence pathway is selected and the isolropic evolution due to 2nd-order quadrupolar shift and the isotropic chemical shift is preserved. To get, rid of the anisotropic terms, $\theta_{1}$ and $O_{2}$ are chosen to have opposite signs for $P_{2}\left(\cos O_{1}\right)$ and $P_{2}\left(\cos O_{2}\right)$, and for $P_{4}\left(\cos \theta_{1}\right)$ and $P_{1}\left(\cos \theta_{2}\right)$.

$$
\begin{aligned}
& P_{2}\left(\cos \theta_{1}\right)=-k_{1} P_{2}\left(\cos \theta_{2}\right) \\
& P_{1}\left(\cos \theta_{1}\right)=-k_{2} P_{4}\left(\cos \theta_{2}\right)
\end{aligned}
$$

It is interesting to see what: happens when $k_{1}=k_{2}=k>0$. In this special case, if another function on $\alpha^{Q}$ and $\beta^{Q}$ is drfined as

$$
A^{Q}\left(\alpha^{Q}, \beta^{Q}\right)=A_{2}^{Q}\left(\alpha^{Q}, \beta^{Q}\right) P_{2}\left(\cos 0_{2}\right)+A_{4}^{Q}\left(\alpha^{Q}, \beta^{Q}\right) P_{4}\left(\cos 0_{2}\right)
$$

Eqn 3.11 and Eq̣ 3.12 can be rewrilten as

$$
\begin{aligned}
& \omega_{1}^{2 Q}\left(\alpha^{Q}, \beta^{Q}\right)=\omega_{i s o}^{2 Q}-k \cdot 1^{Q}\left(\alpha^{Q}, \beta^{Q}\right) \\
& \omega_{2}^{2 Q}\left(\alpha^{Q}, \beta^{Q}\right)=\omega_{i s o}^{2 Q}+A^{Q}\left(\alpha^{Q}, \beta^{Q}\right) .
\end{aligned}
$$

The phase factor experienced by a quadrupolar spin at the start of the acquisition time is

$$
\begin{aligned}
\phi^{2 Q}\left(t_{1}\right) & =\int_{0}^{\frac{t_{1}}{k+1}} \omega_{1}^{2 Q}\left(\Omega^{Q} \cdot \beta^{Q}\right) d l+\int_{0}^{\frac{k t_{1}}{k+1}} \omega_{2}^{2 Q}\left(\alpha^{Q}, \beta^{Q}\right) d t \\
& =\omega_{i s \mathrm{v}}^{2 Q}\left(\frac{l_{1}}{1+k}+\frac{k_{1}}{1+k}\right)-A\left(\alpha^{Q}, \beta^{Q}\right)\left(\frac{k l_{1}}{1+k}-\frac{k t_{1}}{1+k}\right) \\
& =\omega_{i s o}^{2 Q} l_{1} .
\end{aligned}
$$




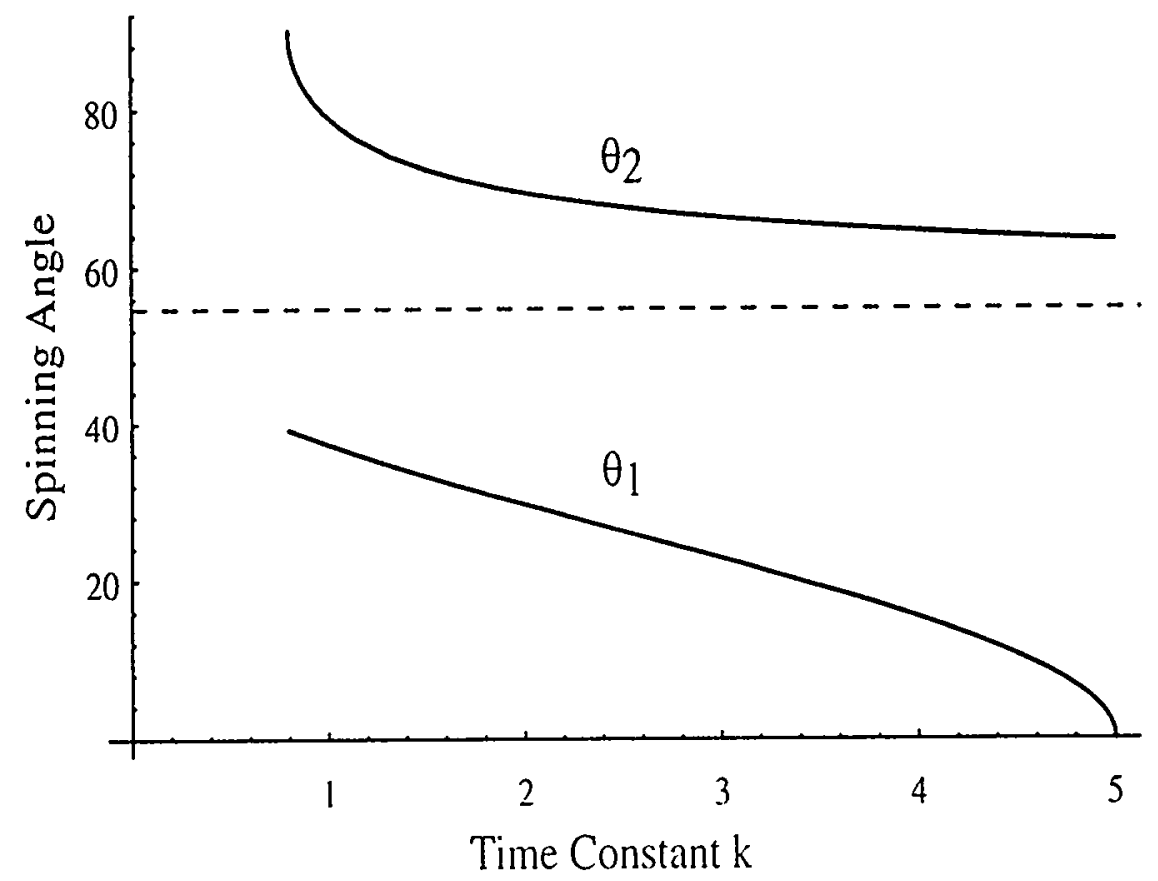

Figure 3.8: DAS angle pairs as a function of $k$. The angle $\theta_{1}$ and $O_{2}$ are solutions to Eqn 3.20 .

The derivation assumes that fhe z-filtor does nothing but restore the density matrix.

The assumption that $k_{1}=h_{2}=k$ leads to,

$$
\frac{P_{2}\left(\cos \theta_{2}\right)}{P_{2}\left(\cos \theta_{1}\right)}=\frac{P_{1}\left(\cos \theta_{2}\right)}{P_{1}\left(\cos \theta_{1}\right)}
$$

whose solution gives $0 . S \leq k \leq 5$. Figure $3 . S$ shows 1 he D. $1 . S$ angle pairs as a function of parameter $k$. The most popularly used pairs ane the $k=1\left(37.38^{\circ}\right.$ and $\left.79.19^{\circ}\right)$ and the $k=5\left(0.00^{\circ}\right.$ and $\left(3.3 .13^{\circ}\right)$ pairs [12. 1:3]. The chemical shift anisotropy (CSA) is also averaged out by D.As. 'lo sere this. consider the erolved phase due to the chemical shift interaction.

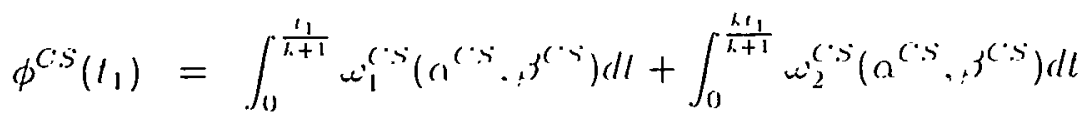

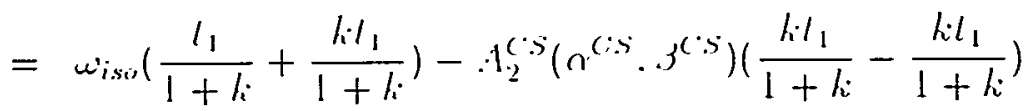

$$
\begin{aligned}
& =\omega_{\text {iss }} t_{1} \text {. }
\end{aligned}
$$


The evolution under the anisolropic chemical shift is refocused al. $l_{1}$. The total phase that a quadrupolar spin experiences after $\iota_{1}$ evolution is

$$
\phi^{D A S}\left(t_{1}\right)=\left(\omega_{i s o}+\omega_{i s o}^{2 Q}\right) t_{1}
$$

and the observed shift in DAS (in the unit of ppm) is

$$
\delta^{D A S}=\delta_{i s o}+\delta_{i s o}^{2 Q}
$$

Here,

$$
\delta_{i s o}^{2 Q}=\frac{10^{6} \cdot \omega_{i s o}^{2 Q}}{\omega_{1}^{2}}
$$

A DAS-spectrum of $\mathrm{RbNO}_{3}$ is shown in Figure 3.9. There are three different rubidium environments in this salt, corresponding to the three isotropic peaks in the DAS spectrum [-14]. Also shown here is the one-dimensional projections in the isotropic (1-D DAS spectrum) and the anisotropic dimensions (VAS spectrum). The projection in the anisotropic dimension matches the VAS spectrum at $79.19^{\circ}$. Since the three rubidium siles arr woll-resolved in the DAS dimension, we can even add up the intensity for each site respectivcly. This gives us three VAS spectra in Figure 3.9, each of which can be sepalately simulated to give quaclrupolar parameters.

\subsubsection{MQMAS}

In DAS, only the spinning angle is rendered to achicre high-resolution. There is another degree of frcedom in Fign 3.4 that is not well-exploited. The quadrupolar Hamiltonian associated with the symmetric multiple-cquantum transition also has a similar format. to that of the central transition. and can be utilized to construct isotropic spectira.

In MQMAS, the sample is continuously spun around the magic-angle. Strong RF pulses (usually with the highest achicvalsle power level, and the pulse is termed as excitation pulse) are applied to crato a l.riplo-quantum coherence from the equilibrated 

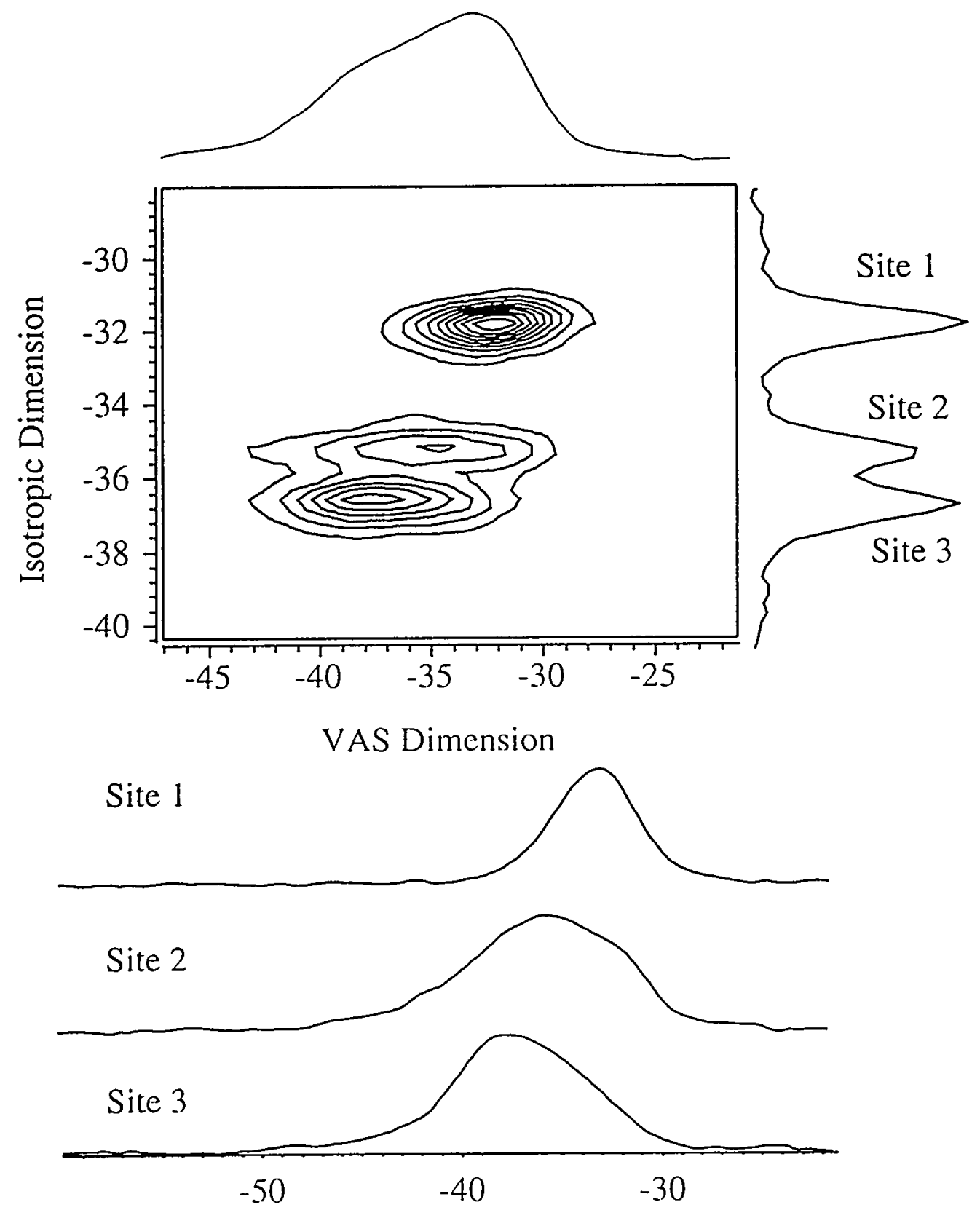

Frequency (ppm from IM RbNO3)

Figure 3.9: Two-dimonsional ${ }^{8 \pi} \mathrm{R}$, 1 ) AS spectrum of $\mathrm{RbNO}_{3}$ and the projections in both dimensions. Single sitce liS spectla for each isol ropic peak in the DAS dimension have been cxtracted and shown at the bottom of the contour plot. 


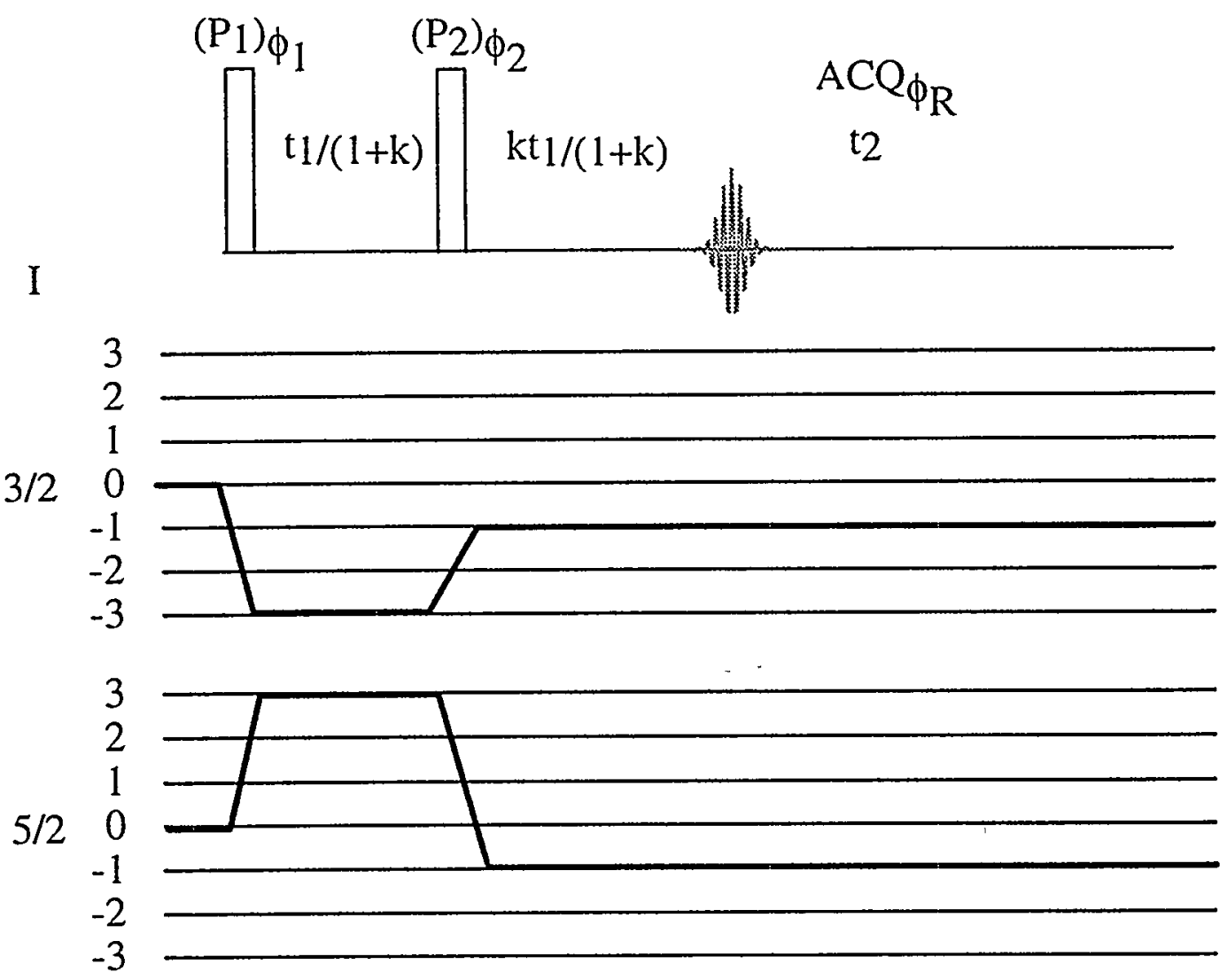

Figure 3.10: MQMAS' experimen1., pulse sequence and coherence pathway. In this experiment, the value $t_{1}$ is incremented in a two-dinensional fashion. The $t_{1}$ dimension reconstructs the isotropic MQMAS spectrum. For spin- $\frac{3}{2}$ nuclei, the $0 \rightarrow-3 \rightarrow-1$ pathway is selected; For spin- $\frac{5}{2}$ nuclei, the $0 \rightarrow 3 \rightarrow-1$ pathway is selected.

spin state $I_{0}$. Such a colicrence evolves under the triple-quantum Hamiltonian for $\frac{t_{1}}{k+1}$, before another pulse (reconversion pulse) is applied to transfer the triple-quantum coherence into single-cuantum coherence. Like DAS. an MQMAS echo is formed at $\frac{k t_{1}}{k+1}$ after this pulse. 'The experiment. pulse sequence and coherence pathway are shown in Figure 3.10 for $l=\frac{3}{2}$ and $l=\frac{5}{2}$ nuclei.

To see how an MQMAS-echo is formed, assume $I=\frac{3}{2}$ and $O=54.74^{\circ}$. The $P_{2^{-}}$ dependent term in $\mathrm{E}$ [n 3.4 is dropped and the frequency expressions for the single- 
and triple-quantum colicrences are

$$
\begin{aligned}
& \omega_{1 / 2 \leftrightarrow-1 / 2}^{2 Q}\left(\alpha^{Q}, \beta^{Q}, \gamma^{Q}\right)=C_{0}(I, 1 / 2) \omega_{i s Q}^{2 Q}+C_{2}(l, 1 / 2) A_{4}^{Q}\left(\alpha^{Q}, \beta^{Q}\right) P_{4}(\cos 0) \\
& \omega_{3 / 2 \leftrightarrow-3 / 2}^{2 Q}\left(\alpha^{Q}, \beta^{Q}, \gamma^{Q}\right)=C_{0}(I, 3 / 2) \omega_{i s o}^{2 Q}+C_{2}(l, 3 / 2) A_{4}^{Q}\left(\alpha^{Q}, \beta^{Q}\right) P_{4}(\cos 0)
\end{aligned}
$$

Assuming that,

$$
k=\left|\frac{C_{2}(I, 3 / 2)}{C_{2}(I, 1 / 2)}\right|,
$$

that $C_{2}\left(I, \frac{3}{2}\right)$ and $C_{2}\left(I, \frac{1}{2}\right)$ have difforent signs (which is true when $I=\frac{3}{2}$ ), and that the $-3 \rightarrow-1$ colicrence pathway is selected, the phase factor experienced by a quadrupolar spin at the start of lhe acquisition is

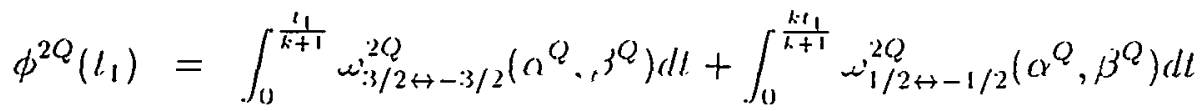

$$
\begin{aligned}
& =\omega_{i s i}^{2 Q}\left(\frac{\left({ }_{0}(I, 3 / 2) l_{1}\right.}{1+l_{i}}+\frac{\left(i_{0}(l, 1 / 2) l_{i} l_{1}\right.}{1+l_{i}}\right) \\
& +P_{1}(\cos \theta) \cdot A_{1}^{Q}\left(\alpha^{Q} \cdot j^{Q}\right)\left(\frac{l_{i}\left(i_{2}(l \cdot 1 / 2) t_{1}\right.}{1+l_{i}}+\frac{\left(C_{2}(l, 3 / 2) t_{1}\right.}{1+k}\right) \\
& =\frac{C_{0}(1,3 / 2)+C_{0}(1,1 / 2) k_{i}}{1+k} \omega_{i s o}^{2 Q} / 1 \\
& =l_{i} \omega_{i s i}^{2 Q} / 1 .
\end{aligned}
$$

For the second to last repualit!:

$$
k=-\frac{\left({ }_{2}(1,3 / 2)\right.}{\left({ }_{2}(1.1 / 2)\right.}
$$

is used. Similar to DAS. Ihe anison roppie phase is cancelled ont al $/$ and an echo is formed. It is however. worth noling that the enolved phase of a quadrupolar spin at $t_{1}$ is no longer $\omega_{i s,}^{2 Q} l_{1}$, bul is $\omega_{i s u}^{2 Q} l_{1}$ scaled by a factor $k_{2}$ defined by

$$
l_{2}=\frac{C_{0}(1.3 / \cdot 2)+C_{0}(1.1 / 2) k_{i}}{1+l_{i}}
$$


The removal of chemical shifl, anisotropy (CSA) by MQMAS is obvious since the sample is rotating around the magic-angle. The frequency shift due to chemical shift interaction is however, different from that in DAS.

$$
\begin{aligned}
\phi^{C S}\left(t_{1}\right) & =\int_{0}^{\frac{t_{1}}{k+1}} \omega_{m \leftrightarrow-m}^{C S}\left(\alpha^{C S}, \beta^{C S}\right) d t+\int_{0}^{\frac{k t_{1}}{k+1}} \omega_{1 / 2 \leftrightarrow-1 / 2}^{C S}\left(\alpha^{C S}, \beta^{C S}\right) d t \\
& =\left(\frac{-2 m}{k+1}+\frac{k}{k+1}\right) \omega_{i s i} t_{1} \\
& =k_{1} \omega_{i s o} l_{1}
\end{aligned}
$$

The fact that the chemical shift of $m \leftrightarrow-m$ transition is $2 m$ times as large as that of the central transition is used in the albove equation. The scaling factor $k_{1}$ is defined by

$$
k_{1}=\frac{-2 m+k}{k+1}
$$

The experimentally observed shift in the isotropic dimension of MQMAS is a combination of the isotropic chmical shift and the 2nd-order quadrupolar shift.

$$
\delta^{M Q M} M A S=k_{1} \delta_{i s o}+k_{2} \delta_{i s o}^{Q}
$$

This shift is different from the DAS shift.

Figure 3.11 show's the MQNAS spectrum of $\mathrm{RbNO}_{3}$, together with the $1 \mathrm{D}$ projections (1D MQMAS and MAS spectra). ('ompared to Figure 3.9, the observed chemical shifts for the three sites are different in both experiments, as a result of the scaling factors ( $k_{1}$ and $\left.k_{2}\right)$.

The assumption that $\left(C_{2}(I, 3 / 2)\right.$ and $\left(C_{2}(I, 1 / 2)\right.$ harc opposite signs can be dropped without changing the principles of lhe MQQMAS experiment. If they instead have same sign $\left(I>\frac{3}{2}\right)$, whe $0 \rightarrow+3 \rightarrow-1$ coherence pallhway can be chosen, to remove the anisotropic part of the ?nd-order cluadrupolar interaction. This is shown in Figure 3.10. The phase cycle that select.s the appropriate coherence pathway is discussed in the next section. 

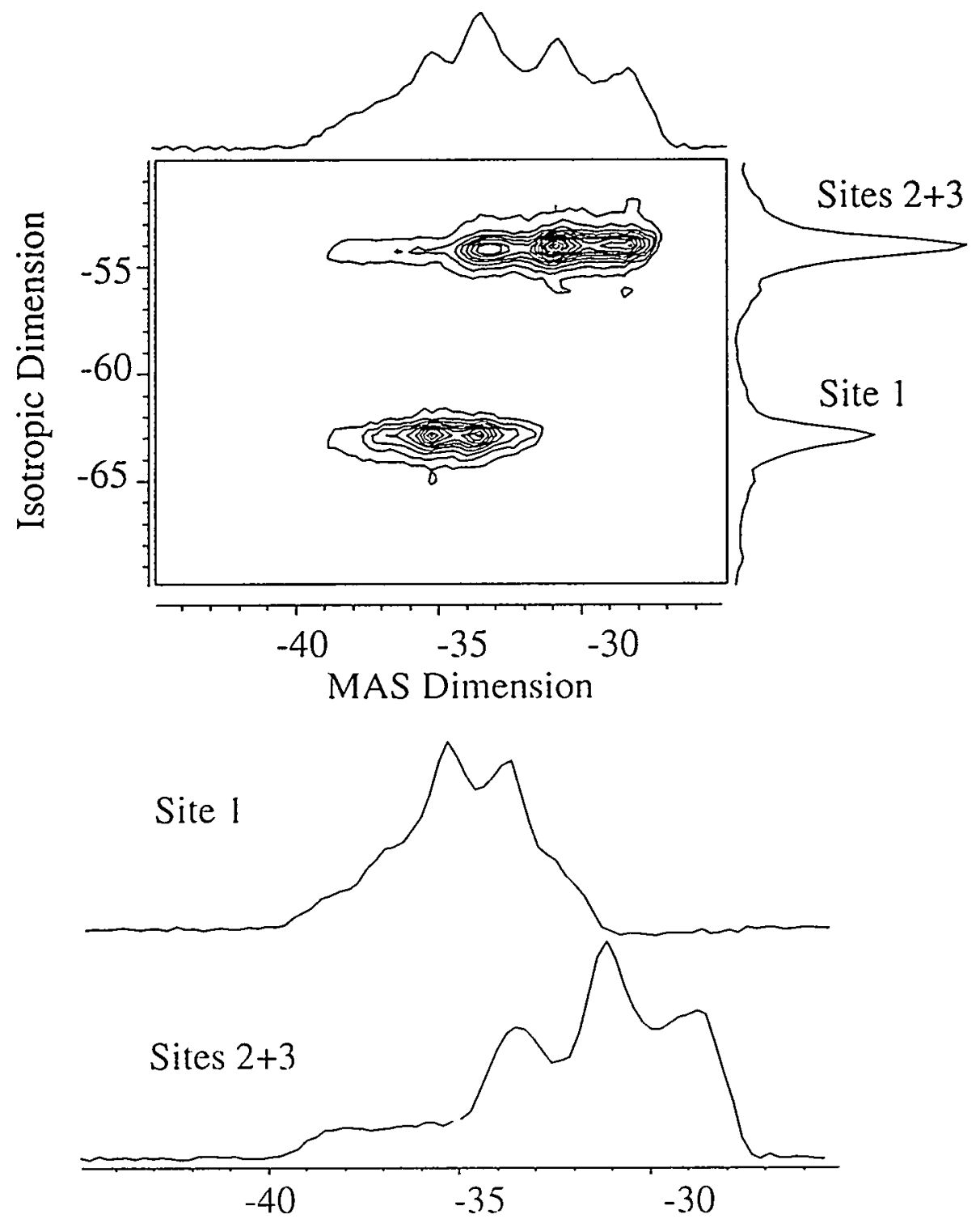

Frequency (ppm from $1 \mathrm{M} \mathrm{RbNO}_{3}$ )

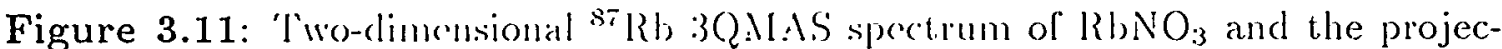
tions in bouh dimonsions. Single sitc MAS spectra for each isotropic peak in the MQMAS dimension have becn extracted and shown at bottom of the contour plot. Notice that sile 2 and 3 in the D.SS spectrum ale not resolved in the MQMAS spectrum. 


\begin{tabular}{cccccc}
\hline$I$ & $m$ & Coherence Pathway & $h$ & $k_{1}$ & $k_{2}$ \\
\hline $3 / 2$ & $1 / 2$ & - & - & - & - \\
& $3 / 2$ & $0 \rightarrow-3 \rightarrow-1$ & $7 / 9$ & $17 / 8$ & $-5 / 4$ \\
$5 / 2$ & $1 / 2$ & - & & & - \\
& $3 / 2$ & $0 \rightarrow 3 \rightarrow-1$ & $19 / 12$ & $-17 / 31$ & $10 / 31$ \\
& $5 / 2$ & $0 \rightarrow-5 \rightarrow-1$ & $25 / 12$ & $85 / 37$ & $-50 / 37$ \\
$7 / 2$ & $1 / 2$ & & & & \\
& $3 / 2$ & $0 \rightarrow 3 \rightarrow-1$ & $101 / 45$ & $-17 / 73$ & $10 / 73$ \\
& $5 / 2$ & $0 \rightarrow 5 \rightarrow-1$ & $11 / 9$ & $-17 / 10$ & 1 \\
& $7 / 2$ & $0 \rightarrow-7 \rightarrow-1$ & $161 / 45$ & $148 / 103$ & $-140 / 103$ \\
$9 / 2$ & $1 / 2$ & & & & - \\
& $3 / 2$ & $0 \rightarrow 3 \rightarrow-1$ & $91 / 36$ & $-17 / 127$ & $10 / 127$ \\
& $5 / 2$ & $0 \rightarrow 5 \rightarrow-1$ & $95 / 36$ & $-13 / 131$ & $50 / 131$ \\
& $7 / 2$ & $0 \rightarrow 7 \rightarrow-1$ & $7 / 18$ & $-47 / 25$ & $14 / 5$ \\
& $9 / 2$ & $0 \rightarrow-9 \rightarrow-1$ & $31 / 6$ & $79 / 55$ & $-50 / 37$ \\
\hline
\end{tabular}

Table 3.1: Coherencr pathway and different scaling values for different spins.

Even though the above discussion focuses on the combination of triple- and singlequantum transitions, othel multiple-cyantum transitions $(5 / 2 \leftrightarrow-5 / 2$, etc.) can also be exploited. The $k$ (Eqn 3.27), $k_{1}$ (Eqn 3.33) and $k_{2}\left(E_{q} n 3.31\right)$ values are different for different transilions. These values. together with the relevant coherence pathways that are chosen to achieve selective average are shown in Table 3.1 [45].

\subsection{Phase Cycle}

The acquisition schemes shown in figures 3.7 and 3.10 do not lead to pureabsorption phase DAS or MQMAS spectra cven if hypercomplex datasets are acquired. Recall from chapter 2 that mirror image cohrence pathways are required to give pure-absorption phase two-dimensional spectra. For DAS, the \pm 1 pathways are to be retained in both halves of the 1 , evolution time. For MQMAS, the \pm 3 pathways 
are to be retained in the first. half. and the \pm 1 pathways are necessary in the second half of the $t_{1}$ evolution. In the original sequences, the receiver detects only the -1 coherence during the scoond hall, thus the detected signal is [1]

$$
\begin{aligned}
& S_{c}\left(t_{1}, l_{2}\right)=e^{-\left(t_{1}+t_{2}\right) / T_{2}} \cos \left(\Omega_{1} l_{1} / 2\right) c^{i \Omega_{2} t_{1} / 2} e^{i \Omega_{2} t_{2}} \\
& S_{s}\left(l_{1}, l_{2}\right)=e^{-\left(t_{1}+l_{2}\right) / T_{2}} \sin \left(\Omega_{1} l_{1} / 2\right) e^{i \Omega_{2} t_{1} / 2} e^{i \Omega_{2} t_{2}} .
\end{aligned}
$$

It is not possible to get purc-absorption plase spectra from these two datasets.

The first modification to D. 1 S to overcome this problem is to have a $z$-filter at the time the DAS-coho forms $[16,-17]$. The filtor mixes $I_{+}$and $I_{-}$coherences and stores the magnetization along z-axis and later restores it. 'This allows the retention of \pm 1 coherences in boll halves of the $l_{1}$ poriod. The method has also been applied to MQMAS by Amoureux [1s] and Wimperis [49]. While pure-absorption phase lineshape is obtained, one half of the signal is lost due to the use of z-filter. A better approach that is used in our exporimenll was first proposed by Girandenetti [50] to get pure-phase DASS speretra: a similar approach is also useful for MQMAS [51]. Figure 3.12 shows the modified DAS and MQNASS sequences. The major diflerence between the new sogucouces and the original ones is that the acquisition starts right after the last pulse. This corresponds to a redefinition of $l_{1}$ and $l_{2}$. For DAS, the evolution at the first angle is now $l_{1}$ and the evolution at the second angle is $t_{2}$.

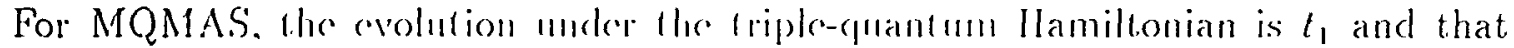

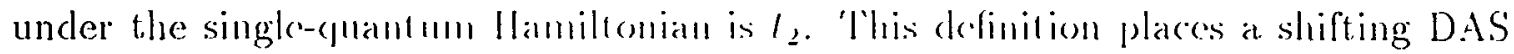
(MQMAS) echo in the /, dimension al time /il, Mhen the data is processed like other two-dimensional data wilhonl modification. a park with a slope $k$ is observed. This peak correlates the firequencies in 1 wo dimensions. A comrontional D.1S (MQMAS) spectrum can be olvained by shearing this spectrum will angle $0[52,5: 3,54]$.

$$
0=1 a n^{-1} /
$$


(a)
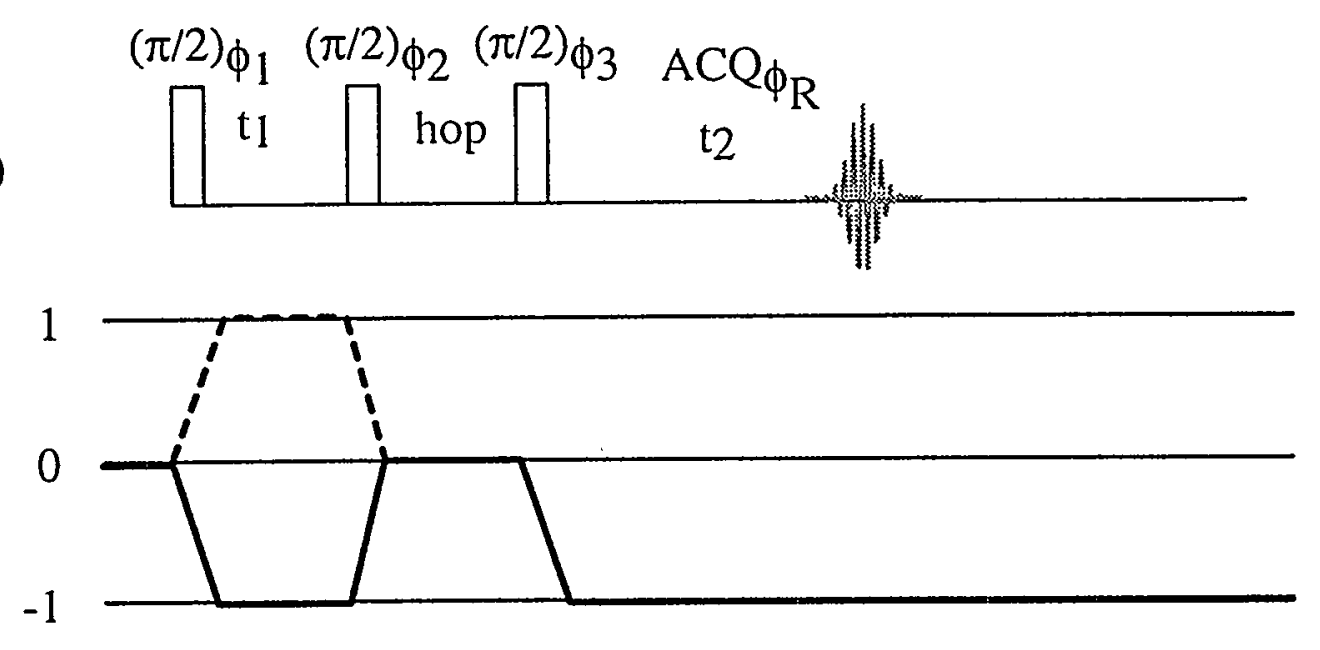

(b)
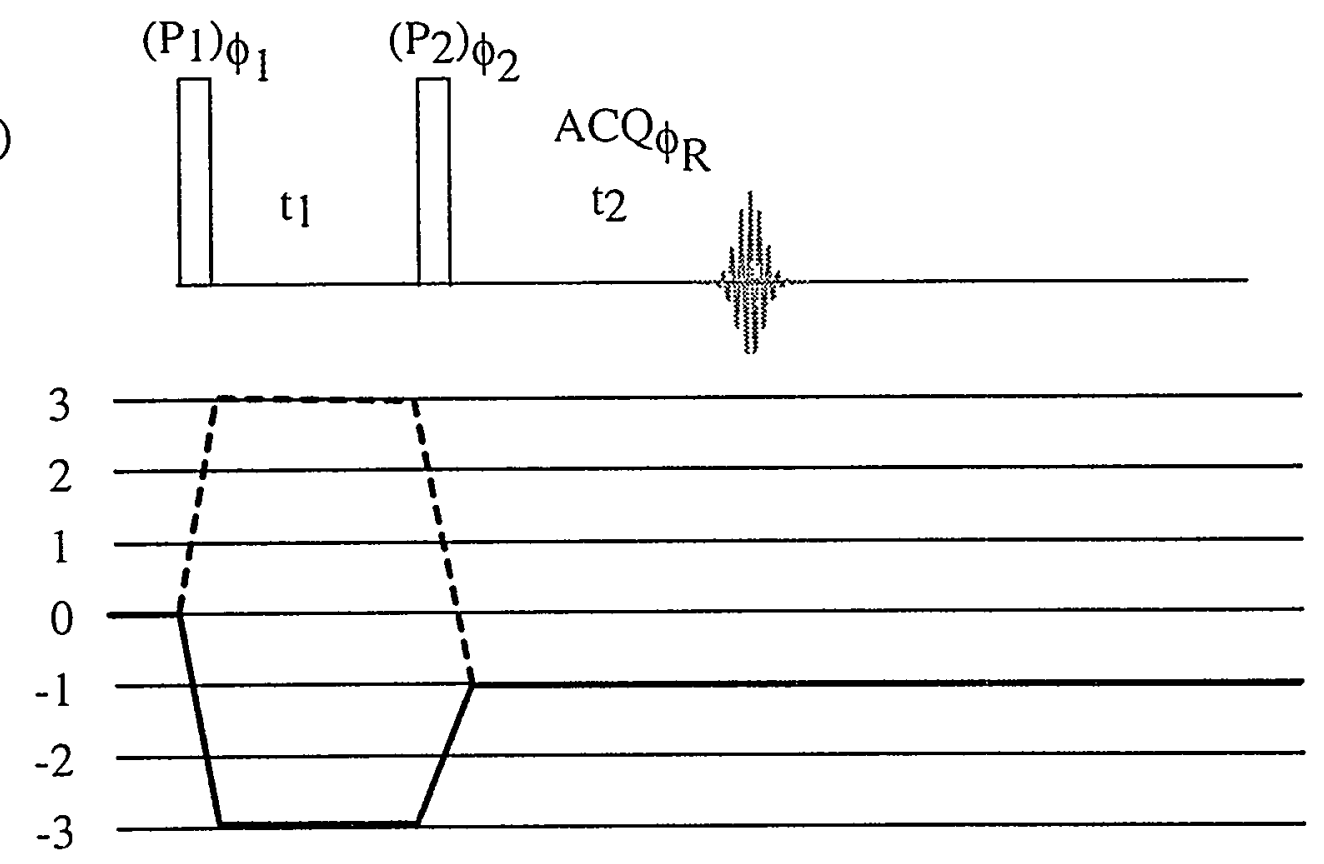

Figure 3.12: Hypercomplex DAS and MQMAS experiment. Coherence pathways designated by the bolded and dashed lines are both relained. 


\begin{tabular}{c|cccc}
\hline \multicolumn{5}{c}{ D $\Lambda S^{\circ}$ cosine } \\
\hline$\phi_{1}$ & $0^{\circ}$ & $0^{\circ}$ & $180^{\circ}$ & $180^{\circ}$ \\
$\phi_{2}$ & $0^{\circ}$ & $180^{\circ}$ & $0^{\circ}$ & $180^{\circ}$ \\
$\phi_{3}$ & $0^{\circ}$ & $0^{\circ}$ & $0^{\circ}$ & $0^{\circ}$ \\
$\phi_{r}$ & $0^{\circ}$ & $180^{\circ}$ & $180^{\circ}$ & $0^{\circ}$ \\
\hline \multicolumn{5}{c}{ DAS sine } \\
\hline$\phi_{1}$ & $90^{\circ}$ & $90^{\circ}$ & $270^{\circ}$ & $270^{\circ}$ \\
$\phi_{2}$ & $0^{\circ}$ & $180^{\circ}$ & $0^{\circ}$ & $180^{\circ}$ \\
$\phi_{3}$ & $0^{\circ}$ & $0^{\circ}$ & $0^{\circ}$ & $0^{\circ}$ \\
$\phi_{r}$ & $0^{\circ}$ & $180^{\circ}$ & $180^{\circ}$ & $0^{\circ}$ \\
\hline
\end{tabular}

Table 3.2: Hypercomplex DAS phase cycle with redefinition of $t_{1}$ and $t_{2}$.

This definition of llor lime axes is very similar to the definitions in other twodimensional experiments. IIsing the rules in chapter two, phase cycles for DAS and MQMAS can be derived (Tables 3.2 and 3.3). For DAS, the derivation has already been worked out in chapter 2 for the cosinc part of the signal (Eqn 2.127). The sine part of the signal is oblained by adding a $90^{\circ}$ phase shift to the first pulse, and keeping all other pulses with their original plases. The phase cycle is shown in Table 3.2. For MQMAS. the change of the coherence order $\Delta p$ is \pm 3 during the first pulse thus a six-step phase cycle is noeded to solect these two coherences. The last pulse is left uncycled so the totat number of steps in a cycle is 6. The receiver phase is set by

$$
o_{r}=-30_{1}+13_{2}
$$

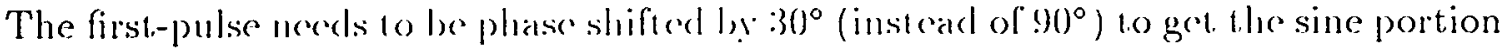
of the signal because llo lriple-quantum cohorence experiences a phase shift three times that of the single-guantum coherence aftor a pulse applied to $/ 0$. The phase cycle is shown in Tablo :3.3.

Most of the spectra presented in this thesis. are however, obtained using whole- 


\begin{tabular}{c|cccccc}
\hline \multicolumn{7}{c}{ 3QMAS cosine } \\
\hline$\phi_{1}$ & $0^{\circ}$ & $60^{\circ}$ & $120^{\circ}$ & $180^{\circ}$ & $240^{\circ}$ & $300^{\circ}$ \\
$\phi_{2}$ & $0^{\circ}$ & $0^{\circ}$ & $0^{\circ}$ & $0^{\circ}$ & $0^{\circ}$ & $0^{\circ}$ \\
$\phi_{r}$ & $0^{\circ}$ & $180^{\circ}$ & $0^{\circ}$ & $180^{\circ}$ & $0^{\circ}$ & $180^{\circ}$ \\
\hline \multicolumn{7}{c}{$3 \mathrm{QMAS} \operatorname{sine}$} \\
\hline$\phi_{1}$ & $30^{\circ}$ & $90^{\circ}$ & $150^{\circ}$ & $210^{\circ}$ & $270^{\circ}$ & $330^{\circ}$ \\
$\phi_{2}$ & $0^{\circ}$ & $0^{\circ}$ & $0^{\circ}$ & $0^{\circ}$ & $0^{\circ}$ & $0^{\circ}$ \\
$\phi_{r}$ & $0^{\circ}$ & $180^{\circ}$ & $0^{\circ}$ & $180^{\circ}$ & $0^{\circ}$ & $180^{\circ}$ \\
\hline
\end{tabular}

Table 3.3: Hypercomplex MQMAS phase cycle with redefinition of $t_{1}$ and $t_{2}$. echo acquisition $[50,51,55]$ in which a pulse sequence of the form

$$
\pi / 2-l_{\epsilon}-\pi-a c q
$$

is used to refocus the signal and produce an echo. Here $t_{e}$ is an echo time set to many milliseconds so the acquisilion follows both the formation and decay of the echo. Notice that in an ordinary echo experiment, only the decay of the echo is recorded. The two-dimensional signal is of the form

$$
S\left(l_{1}, l_{2}\right)=e^{-l_{1} / T_{2}} e^{i \Omega_{1} l_{1}} e^{-\left(l_{2}-l_{-}\right)^{2} / T_{2}^{2}} e^{i \Omega_{2}\left(l_{2}-l_{n}\right)}
$$

When this signal is Fouricr lansformed with respect to $l_{2}$ and then phase corrected by a time $t_{c}$ to the w.2 dimension. Ihe following signal is obtained,

$$
S\left(l_{1}, u_{2}\right)=c^{-l_{1} / T_{2}} e^{i \Omega_{1} t_{3}} A_{s r}\left(u_{2}^{\prime}, \Omega_{2}\right)
$$

where

$$
A_{s r}\left(\omega_{2} \cdot \Omega_{2}\right)=c^{-\left(\omega_{2}-\Omega_{2}\right)^{2} \cdot T_{2}^{2} / 4}
$$

When the $t_{1}$ dimension is also lourier lansformed. purc-absorption mode 2D-spectrum is obtained.

$$
S\left(\omega_{1}, \omega_{2}\right)=A_{s r}\left(\omega_{2}, \Omega_{2}\right)\left(-1\left(\omega_{1}, \Omega_{1}\right)+i D\left(\omega_{1}, \Omega_{1}\right)\right)
$$




\begin{tabular}{l|llllllll}
\hline \multicolumn{7}{c}{ IyperSED AS cosine } \\
\hline$\phi_{1}$ & $0^{\circ}$ & $0^{\circ}$ & $0^{\circ}$ & $0^{\circ}$ & $180^{\circ}$ & $180^{\circ}$ & $180^{\circ}$ & $180^{\circ}$ \\
$\phi_{2}$ & $0^{\circ}$ & $0^{\circ}$ & $0^{\circ}$ & $0^{\circ}$ & $0^{\circ}$ & $0^{\circ}$ & $0^{\circ}$ & $0^{\circ}$ \\
$\phi_{3}$ & $0^{\circ}$ & $90^{\circ}$ & $180^{\circ}$ & $270^{\circ}$ & $0^{\circ}$ & $90^{\circ}$ & $180^{\circ}$ & $270^{\circ}$ \\
$\phi_{r}$ & $0^{\circ}$ & $270^{\circ}$ & $180^{\circ}$ & $90^{\circ}$ & $180^{\circ}$ & $90^{\circ}$ & $0^{\circ}$ & $270^{\circ}$ \\
\hline \multicolumn{7}{c}{ IyperSEDAS sine } \\
\hline$\phi_{1}$ & $90^{\circ}$ & $90^{\circ}$ & $90^{\circ}$ & $90^{\circ}$ & $270^{\circ}$ & $270^{\circ}$ & $270^{\circ}$ & $270^{\circ}$ \\
$\phi_{2}$ & $0^{\circ}$ & $0^{\circ}$ & $0^{\circ}$ & $0^{\circ}$ & $0^{\circ}$ & $0^{\circ}$ & $0^{\circ}$ & $0^{\circ}$ \\
$\phi_{3}$ & $0^{\circ}$ & $90^{\circ}$ & $180^{\circ}$ & $270^{\circ}$ & $0^{\circ}$ & $90^{\circ}$ & $180^{\circ}$ & $270^{\circ}$ \\
$\phi_{r}$ & $0^{\circ}$ & $270^{\circ}$ & $180^{\circ}$ & $90^{\circ}$ & $180^{\circ}$ & $90^{\circ}$ & $0^{\circ}$ & $270^{\circ}$ \\
\hline
\end{tabular}

Table 3.4: Hyperemplex SEDAS phase cycle with redefinition of $t_{1}$ and $t_{2}$.

The advantage of whole-crho acquisition is that it does not require the additional dataset like the States moliod. It l.hus has a factor of $\sqrt{2}$ improvement in signalto-noise ratio. Shifted-cho D) AS (SFI)AS) and shifiod-echo MQMAS (SEMQMAS) were discussed in [50] and [51], respectively: The experiments can be further modified to combine with the States melhod to give HyperSlid)AS and HyperSEMQMAS sequences, which collect also the mitror image colerence in the first half of the $t_{1}$ period and have another factor of $\sqrt{2}$ improvement in signal-to-noise ratio.

The sequences and coherence pathways for the IyperSEDAS and HyperSEMQMAS experiments are shown in Figure 3.1:3. 'lhe phase cycles for these two experiments are given in 'lable :3.1 and 'Table :3.5. For DAS, the first pulse is cycled through two-stops to select llac \pm 1 colerences. 'The third pulse is cycled through 4-steps to select the +1 coherence. For MQXIS, the first pulse is cycled through six-steps to select the \pm 3 colerences. The thied pulse is cycled through 8-steps to select the +1 colicronce. 
(a)
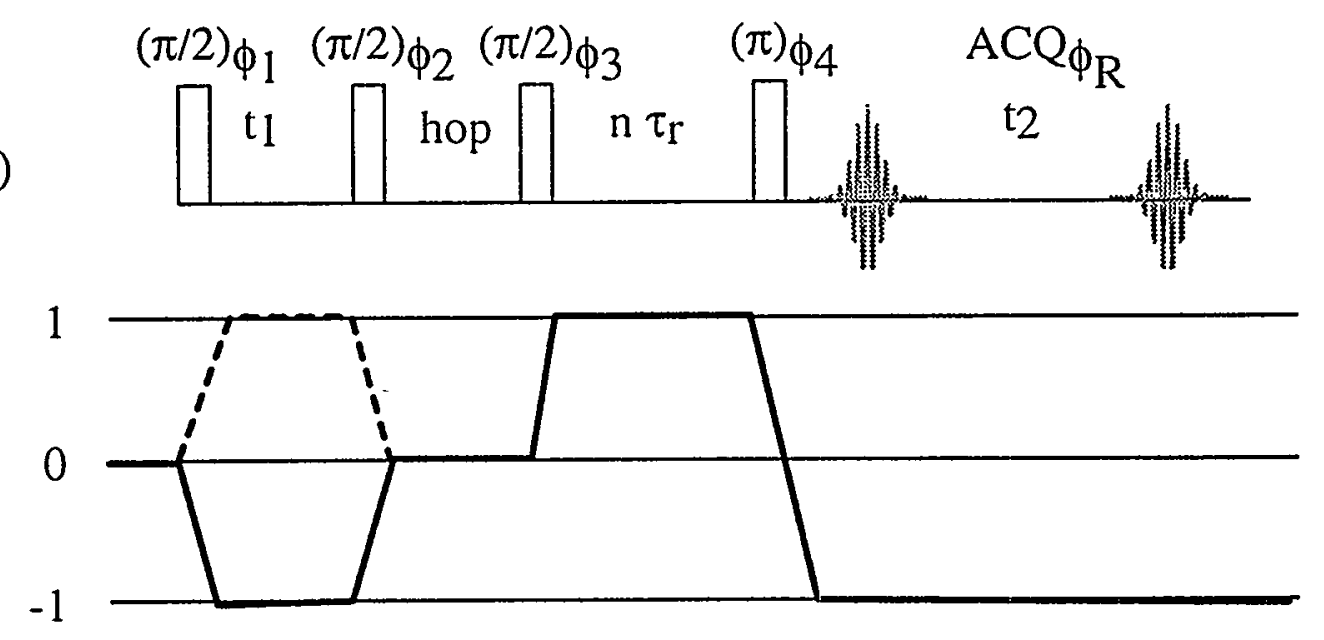

(b)

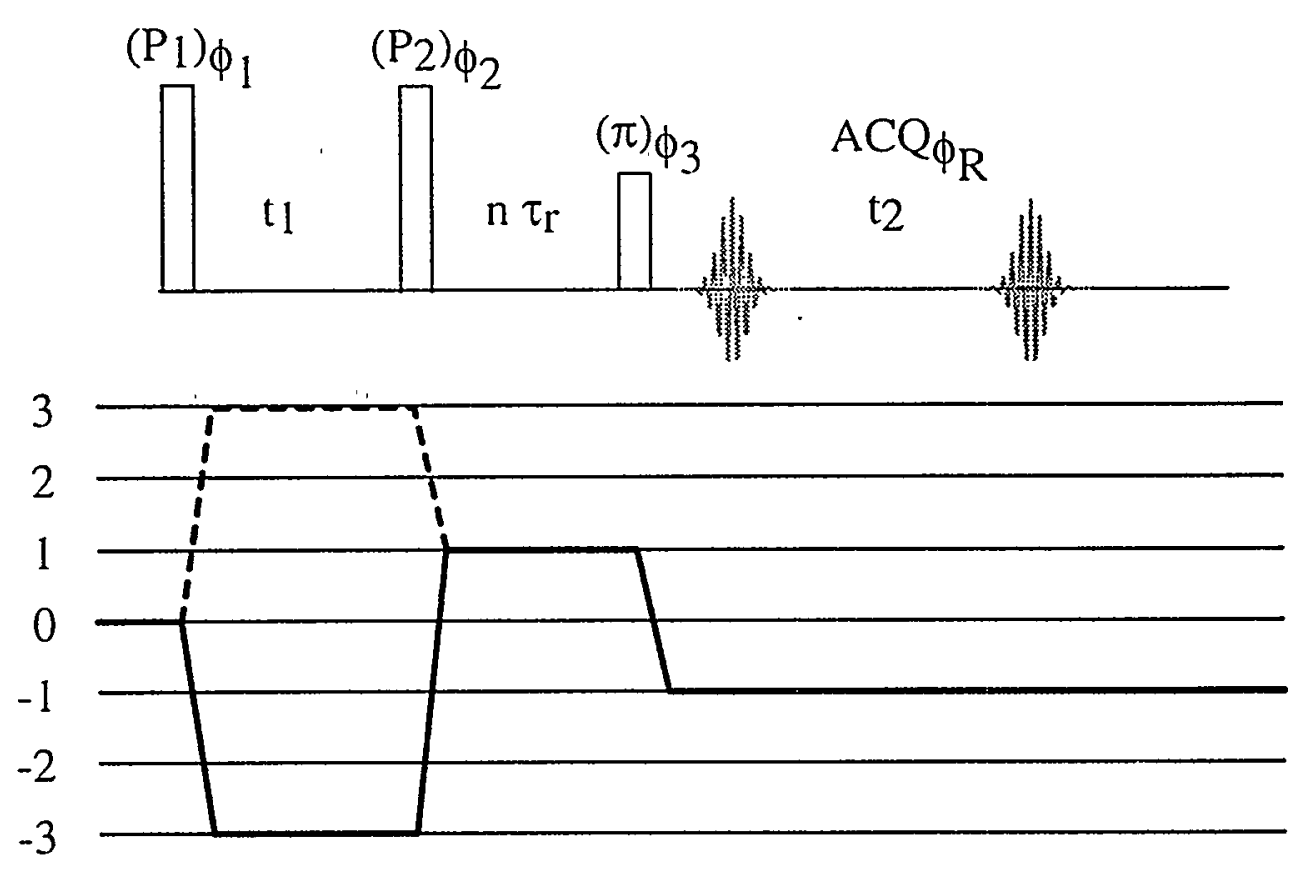

Figure 3.13: HyperSEDAS and Iy'prSLMQNAS cxperiments which acquire whole DAS or MQMAS echo in the $t_{2}$ dimension. 


\begin{tabular}{|c|c|c|c|c|c|c|c|c|c|c|c|c|}
\hline \multicolumn{13}{|c|}{ Iy'perSEMQMAS cosine } \\
\hline$\phi_{1}$ & $0^{\circ}$ & $60^{\circ}$ & $120^{\circ}$ & $180^{\circ}$ & $240^{\circ}$ & $300^{\circ}$ & & & & & & \\
\hline$\phi_{2}$ & $0^{\circ}$ & & & & & & & & & & & \\
\hline \multirow[t]{4}{*}{$\phi_{3}$} & $0^{\circ}$ & $0^{\circ}$ & $0^{\circ}$ & $0^{\circ}$ & $0^{\circ}$ & $0^{\circ}$ & $15^{\circ}$ & $45^{\circ}$ & $45^{\circ}$ & $45^{\circ}$ & $45^{\circ}$ & $45^{\circ}$ \\
\hline & $90^{\circ}$ & $90^{\circ}$ & $90^{\circ}$ & $90^{\circ}$ & $90^{\circ}$ & $90^{\circ}$ & $135^{\circ}$ & $135^{\circ}$ & $135^{\circ}$ & $135^{\circ}$ & $135^{\circ}$ & $135^{\circ}$ \\
\hline & $180^{\circ}$ & $180^{\circ}$ & $180^{\circ}$ & $180^{\circ}$ & $180^{\circ}$ & $180^{\circ}$ & $22.5^{\circ}$ & $225^{\circ}$ & $225^{\circ}$ & $22.5^{\circ}$ & $225^{\circ}$ & $225^{\circ}$ \\
\hline & $270^{\circ}$ & $270^{\circ}$ & $270^{\circ}$ & $270^{\circ}$ & $270^{\circ}$ & $270^{\circ}$ & $315^{\circ}$ & $315^{\circ}$ & $315^{\circ}$ & $315^{\circ}$ & $315^{\circ}$ & $315^{\circ}$ \\
\hline \multirow{2}{*}{$\phi_{r}$} & $0^{\circ}$ & $180^{\circ}$ & $0^{\circ}$ & $180^{\circ}$ & $0^{\circ}$ & $180^{\circ}$ & $90^{\circ}$ & $270^{\circ}$ & $90^{\circ}$ & $270^{\circ}$ & $90^{\circ}$ & $270^{\circ}$ \\
\hline & $180^{\circ}$ & $0^{\circ}$ & $180^{\circ}$ & $0^{\circ}$ & $180^{\circ}$ & $0^{\circ}$ & $270^{\circ}$ & $90^{\circ}$ & $270^{\circ}$ & $90^{\circ}$ & $270^{\circ}$ & $90^{\circ}$ \\
\hline \multicolumn{13}{|c|}{ IyperSEMQMAS sine } \\
\hline$\phi_{1}$ & $30^{\circ}$ & $90^{\circ}$ & $150^{\circ}$ & $210^{\circ}$ & $270^{\circ}$ & $330^{\circ}$ & & & & & & \\
\hline$\phi_{2}$ & $0^{\circ}$ & & & & & & & & & & & \\
\hline \multirow[t]{4}{*}{$\phi_{3}$} & $0^{\circ}$ & $0^{\circ}$ & $0^{\circ}$ & $0^{\circ}$ & $0^{\circ}$ & $0^{\circ}$ & $45^{\circ}$ & $45^{\circ}$ & $45^{\circ}$ & $45^{\circ}$ & $45^{\circ}$ & $45^{\circ}$ \\
\hline & $90^{\circ}$ & $90^{\circ}$ & $90^{\circ}$ & $90^{\circ}$ & $90^{\circ}$ & $90^{\circ}$ & $135^{\circ}$ & $1355^{\circ}$ & $1335^{\circ}$ & $1355^{\circ}$ & $135^{\circ}$ & $135^{\circ}$ \\
\hline & $180^{\circ}$ & $180^{\circ}$ & $180^{\circ}$ & $180^{\circ}$ & $180^{\circ}$ & $180^{\circ}$ & $2255^{\circ}$ & $225^{\circ}$ & $225^{\circ}$ & $225^{\circ}$ & $225^{\circ}$ & $225^{\circ}$ \\
\hline & $270^{\circ}$ & $270^{\circ}$ & $270^{\circ}$ & $270^{\circ}$ & $2 \pi 0^{\circ}$ & $270^{\circ}$ & $315^{\circ}$ & $315^{\circ}$ & $315^{\circ}$ & $315^{\circ}$ & $315^{\circ}$ & $315^{\circ}$ \\
\hline \multirow[t]{2}{*}{$\phi_{r}$} & $0^{\circ}$ & $180^{\circ}$ & $0^{\circ}$ & $180^{\circ}$ & $0^{\circ}$ & $180^{\circ}$ & $90^{\circ}$ & $270^{\circ}$ & $90^{\circ}$ & $270^{\circ}$ & $90^{\circ}$ & $270^{\circ}$ \\
\hline & $180^{\circ}$ & $0^{\circ}$ & $180^{\circ}$ & $0^{\circ}$ & $180^{\circ}$ & $0^{\circ}$ & $270^{\circ}$ & $90^{\circ}$ & $270^{\circ}$ & $90^{\circ}$ & $270^{\circ}$ & $90^{\circ}$ \\
\hline
\end{tabular}

Table 3.5: Hypercomplex SLMQMAS phase cycle with redefinition of $t_{1}$ and $t_{2}$.

\subsection{Excitation and Reconversion Pulses}

The preceding section on MQMAS marle an assumption that a single-RF pulse applied on resonance is capable of exciting mulliple-cluantum coherence and transferring it back to single-gluantum colverence for detection. In fact, the behavior of the quadrupolar spin system subject to strong $R F^{\circ}$ pulses needs to be carefully reconsidered here to see how ho excilation and reconersion happent. Some important questions to be answered ale:

I. With what efficiency can mult ipheremantum colverence be excited?

II. How efficiently can mult iple-guanu un colerence be reconverted into singlequantum colicrence?

III. How do mulliple-cluantum colecrences evolve? 
IV. How do the phases of the RF-pulses affect the phase of the signal?

V. For a powder sample, are the excitation and reconversion processes homogeneous? That is, are crystallites with different orientations equally excited? As a result. is the resultant lineshape in the MAS dimension the same as ordinary MAS lineshapes?

\section{Is MQMAS quantitative?}

Experimental answers to the above questions are summarized here. Relatively good signal-to-noise ralios can be achieved for nuclei with high $\gamma$, high abundance and short spin-lattice relaxation time $[15,56,57,58,59,60,61,62]$. The Excitation and reconversion are expected to be inhomogeneous, but experimental results seem to disagree with this since good culadrupolar lineshapes arc often observed in the MAS dimension when the quadmpolar compling constant are not, too large [58]. MQMAS is not quantitalive, at. Ieast. for the case that a single pulse is used for excitation. Relatively quantitative results, however, were presented recently by Griffin et al. [63].

\subsubsection{Fictitious Spin- $\frac{1}{2}$ Operators}

For the description of a spin- $\frac{1}{2}$ system (or the central transition of a quadrupolar nuclear spin system), l.1ner special operat ors $I_{0}, I_{ \pm}$are introduced. Together with the unity operalor 1, the four operators form a complete basis that the clensity matrix can be expressed as

$$
\rho(l)=a_{0}(l) l_{0}+a_{1}(l) l_{+}+a_{-1}(t) l_{-}+a_{2} l .
$$

The commutalion relations annong these operators often allow the analytical solution of the evolution of the density ntat rix of the system. More importantly, the effects 
of RF-pulses and frec induction decay on the spin syestem can be described in an intuitive way (See section 2.5.2).

In the general case of $I>1 / 2$ spins, there are $2 I+1$ eigenstates and other operators are necded. Suppose llat we have $N$ llermitian operators $A_{n}$, the density matrix and Hamiltonian are then expressed as

$$
\begin{aligned}
\rho(l) & =\sum_{n=1}^{N} a_{n}(l) \cdot t_{n}+a_{0} l \\
I I & =\sum_{n=1}^{N} h_{n}(l) \cdot A_{n}
\end{aligned}
$$

analogous to the spin- $\frac{1}{2}$ atse. 'The $N$ opreators should be chosen to fulfill certain Cartesian commutation mations whicht facilitate the calculation of the evolution of the system.

$$
\rho(l)=c^{-t l l t} \rho(0) e^{i l l t}
$$

One way of choosing ho operators is to clofinc fictitious spin- $\frac{1}{2}$ operators for a quadrupolar system. Whan $l=\frac{3}{2}$. the cigenstates $\left|\frac{3}{2}>,\right| \frac{1}{2}>,\left|-\frac{1}{2}>,\right|-\frac{3}{2}>$ can be renamed as $|4>|:, 3>, \mid 2>$ and $\mid 1>$. For cach pair of the cigenstates, we introduce three operators according to the thres Pauli matrices $\sigma_{x}, \sigma_{n}$ and $\sigma_{z}$.

$$
\begin{aligned}
& I_{r}^{r s}=\frac{1}{2}(|r><. s|+|. s><r|) \\
& l \cdot s=-\frac{i}{\underline{2}}(|r><s|-\mid . s>\langle r|)
\end{aligned}
$$

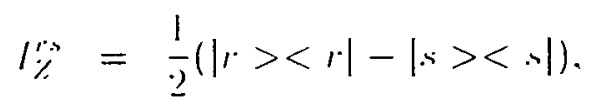

where

$$
\left\langle r\left|I_{x}^{r s}\right| \cdot s>=\frac{1}{2}\right.
$$




$$
\begin{aligned}
& \left\langle r\left|I_{Y}^{r s}\right| s\right\rangle=-\frac{i}{2} \\
& \left\langle r\left|I_{Z}^{r s}\right| s\right\rangle=\frac{1}{2} .
\end{aligned}
$$

The commutation relations among these operators are described below.

$$
\begin{gathered}
{\left[I_{X}^{r s}, l_{Y}^{r s}\right]=i I_{Z}^{r s}} \\
{\left[I_{X}^{r s}, I_{X}^{s t}\right]=\frac{i}{2} I_{Y}^{r t}} \\
{\left[I_{Y}^{r s}, I_{Y}^{s t}\right]=-\frac{i}{2} I_{Y}^{r t}} \\
{\left[I_{Z}^{r s}, I_{Z}^{s t}\right]=0} \\
{\left[I_{X}^{r s}, I_{Y}^{s t}\right]=-\frac{i}{2} I_{X}^{r !}}
\end{gathered}
$$

Other commutation relalions wan be found in $[64,6.5]$. The above relations can be generalized as

$$
[P, Q]=i \kappa R
$$

where $P, Q$, and $R$ are threc operators in Ecns 3.54 through 3.58 . Notice that these operators are not totally independent. For example,

$$
I_{Z}^{r s}=I_{Z}^{r \prime}+I_{\%}^{I s}
$$

In principle, only $(2 /+1)^{2}-1$ independent operators are needed for a spin-I system.

The observable opcrators $I_{X} . I_{Y} . I_{\%}$ (an bo expressed as the summation of the above operators. For a spin-l syest.cin.

$$
\begin{aligned}
I_{X . Y} & =\left.\sum_{r \cdot s} \sqrt{c(r \cdot s)}\right|_{x \cdot y} ^{r s s} \\
I_{y} & =\sum_{r \cdot s} c^{(r \cdot s)} I_{Z}^{r s s}
\end{aligned}
$$


and

$$
c^{(r s)}=I(I+1)-m_{r} \cdot m_{s}
$$

Rotations in the spin space by RF pulses are then clescribed by

$$
{ }^{-O O P} Q C^{i O P}=Q \cos \kappa O+R \sin \kappa O
$$

For example, from Egyn :3.56, we get.

$$
I_{Y}^{r s}(-0) I_{Y}^{s t} I_{Y}^{r s}(0)=I_{Y}^{s t} \cos (0 / 2)-I_{Y}^{r} \sin (0 / 2)
$$

with

$$
I_{p}^{r \cdot s}(0)=r^{20 I_{r}^{r *}}
$$

where $p=. X^{\prime}, Y, \%$.

\section{- 3.4.2 Excitation by Single Pulse}

The Hamilonian of a spin- $\frac{3}{2}$ system is expressed by

$$
\begin{aligned}
H & =H_{\nu J S}+H_{Q}+H_{R I} \\
& =-\Delta \omega\left(: 3 I_{Z}^{1 \cdot 4}+I_{Z}^{2 \cdot 3}\right)+\omega_{Q}\left(I_{Z}^{12}-I_{Z}^{3 \cdot 1}\right)-\omega_{1}\left(\sqrt{3} I_{X}^{12}+2 I_{X}^{23}+\sqrt{3} I_{X}^{34}\right),
\end{aligned}
$$

where $H_{\text {off }}$ is the frepurney offset and inchudes the chomical shift. We have used

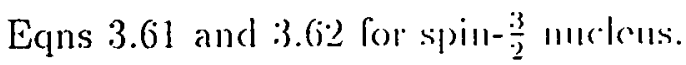

$$
\begin{aligned}
& I_{x}=\sqrt{3}\left(I_{x}^{12}+I_{x^{3}}^{3 \cdot 1}\right)+2 I_{x}^{23} \\
& l==\sqrt{3}\left(l_{1}^{1 \cdot 2}+l_{1}^{3 \cdot 1}\right)+2 l_{5}^{23} \\
& I_{\%}=31 \frac{1}{\%}+I_{z}^{\prime 3}
\end{aligned}
$$

Here, the 2nd-order quadrupolar intoraction has beron neglected. If we also take $\Delta \omega=0$ and assillmo $\omega_{1} \ll \omega_{Q} Q \cdot \| r g \times$

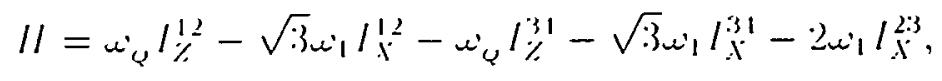


where the first two terms commute with the next wo terms. Wc can transform the above Hamiltonian into a lilted frame by the following operator,

$$
U_{Y}^{12}(\theta) U_{Y}^{3 \cdot 1}(-0)
$$

where

$$
0=\tan ^{-1}\left(\sqrt{3} \omega_{1} / \omega_{Q}\right)
$$

The resultant Iamiltonian is then

$$
\begin{aligned}
H_{T} & =\omega_{c}\left(I_{Z}^{12}-I_{Z}^{31}\right)-2 \omega_{1} I_{Y}^{12}(-0) U_{Y}^{3 \cdot 1}(0) I_{X}^{23}\left(I_{Y}^{3.4}(-0) U_{Y}^{12}(0)\right. \\
& =\omega_{c}\left(I_{Z}^{12}-I_{Z}^{3 \cdot 1}\right)-2 \omega_{1}\left(\cos ^{2} \frac{0}{2} I_{X}^{2 \cdot 3}+\sin ^{2} \frac{0}{2} I_{X}^{1.4}+\frac{1}{2} \sin 0\left(I_{X}^{2.4}+I_{X}^{13}\right)\right),
\end{aligned}
$$

where

$$
\omega_{c}=\frac{1}{\sqrt{\omega_{Q}^{2}+3 \omega_{1}^{2}}}
$$

and $H_{T}$ is the Hamiltonian in the lilted fiame. If we also neglect the last term in the equation for $H_{T}$ and assumc $w_{1} \ll w_{Q}$, the tilted Hamiltonian becomes

$$
H_{\gamma} \approx \omega_{Q}\left(I_{Z}^{12}-I_{Z}^{31}\right)-2 \omega_{1} I_{X}^{23}-\frac{3 \omega_{1}^{3}}{2 \omega_{Q}^{2}} I_{X}^{14}
$$

Clearly, the last two terms correspond to the single- and triple-cuantum transition operators. The separation of the last two terms lias the advantage that rotations in single-quant.um and triplo-qua.ul.um sulspaces are independent. The equilibrated density ma.trix

$$
\rho(0) \times 1 \frac{2 \cdot 3}{\%}+3 I_{\%}^{1 \cdot 1}
$$

then evolves under $/ 1 \%$ according to

$$
\begin{aligned}
& \rho(l)=c^{-i \|_{r}} \rho(0) c^{i \|_{r} t} \\
& =\left(I_{\%}^{2: 3} \cos 2 \omega_{1} l+I_{Y}^{23} \sin 2 \omega_{1} l\right) \\
& +3\left(I_{\%}^{1 \cdot 1} \cos \left(\frac{3 \omega_{1}^{3}}{2 \omega_{Q}^{\prime 2}} l\right)+I_{Q}^{1 \cdot 1} \sin \left(\frac{3 \omega_{1}^{3}}{2 \omega_{Q}^{2}} l\right)\right) .
\end{aligned}
$$


This equation can now be used to kescribe the effect of an RP-pulse on a spin- $\frac{3}{2}$ system.

1. The terms in the first parenthesis clescribe the cffect of the pulse on the central transition. This rexpession is almost l.he same compared to the spin- $\frac{1}{2}$ case except, for the cxtra factor of 2 . It suggests that the central transition behaves lilic an isolated fictitious spin- $\frac{1}{2}$ nucleus with a doubled nutation frecpuency. Morr generally, for a spin-/ nucleus, scaling factor for the nutation frepurucy is $l+\frac{1}{2}$.

II. The terms in the second parnthesis describes the effect of the pulse on the triple-cuantum tansition. Il suggests lhat an RP-pulse gemerates a rotation in the triplo-quantum subspace. The mutation frequency is $\omega_{1}$ scaled by a. factor $\left(\frac{3}{2} \frac{w_{1}}{w_{Q}}\right)^{2}$. 'The magnitude of the mutation, however, is two times larger than that of the central transition. This moans that if the triple-cutantum coliernce is fully excitcel, its intensity is even stronger than that of the contral tansition. Experimontally, this is hardly true due to the inefficioncy of the excitation.

III. The triple-guantum mulation fresueney is depenelent on the quadrupolar frequancy,

$$
\omega_{Q}=\frac{C_{Q}}{2 I(2)-1)}\left(\frac{3 \cos ^{2} j^{Q}-1}{2}+\frac{h_{Q}}{2} \sin ^{2} \cdot g^{Q} \cos 2 n^{Q}\right)
$$

which is orientation-elepremalent. This moans llat a $90^{\circ}$ pulse for one crystallite is nol the $900^{\circ}$ pulse for ofher crystallites and a triple-guantum $90^{\circ}$ pulse for a poweler sample can not be delinerl. As a result, the triplequantium excitation is inhomogencous. 
The nutation behavior of a single crystial sample with $\omega_{Q}=400 \mathrm{k} \mathrm{Hz}$ is calculated as a function of RE-pulse length and shown in Figure 3.14a. From the sinusoidal dependence of the excitation cfficiency on the pulse length, it is clear that the excitation is very inhomogencous. When an average over the whole sample is taken, Figure $3.14 \mathrm{~b}$ is obtained, where the triplo-quant.um signal builds up initially and fluctuates with longer pulses. Thic maximum of the excitation occurs when $\omega_{1} t \approx 3 \pi$ from this simple model.

It seems that for a spimning sample. the dorivation above is still qualitatively correct, especially when lhe spinning speed is not very high and the RF power is high (see Figure 3.15). Assuming that $\omega_{1}=100 \mathrm{kH}$ and $\omega_{r}=10 \mathrm{kHz}$, a solid state $3 \pi$ pulse for $I=\frac{3}{2}$ nuclous is alooul $7.5 / 1$ s. which laklies $7.5 \%$ of a rotor period and the spinning effect may be neglecteel. The assumption that $\omega_{1} \ll \omega_{Q}$ is not often true, because $\omega_{Q}$ is orientation-depondent. Nevertheless, the experimental data in Figure 3.15a suggests that spimning offect is not crucial and can be neglected in a qualitative analysis.

\subsubsection{Reconversion by Single Pulse}

To calculate the recouression eflicioncy, we assume that, the initial density matrix is $I_{Y}^{1.4}$ and calculate the colution of the system undor R R-pulse. we then have

$$
\begin{aligned}
& a_{x}^{2 \cdot 3}=\frac{1}{1} \sin ^{2} \theta\left(\cos \omega^{13} l+\cos \omega^{2 \cdot 1} l-2\right) \\
& a_{y}^{1-1}=0
\end{aligned}
$$

where

$$
\begin{aligned}
& \omega^{1: 3}=\left(3 \omega_{1}^{2}+\left(\omega_{Q}^{2}-\omega_{1}\right)^{2}\right)^{1 / 2} \\
& \dot{\omega}^{2 \cdot 1}=-\left(3 \omega_{1}^{2}+\left(\omega_{\varphi}^{2}+\omega_{1}^{\prime}\right)^{2}\right)^{1 / 2} .
\end{aligned}
$$



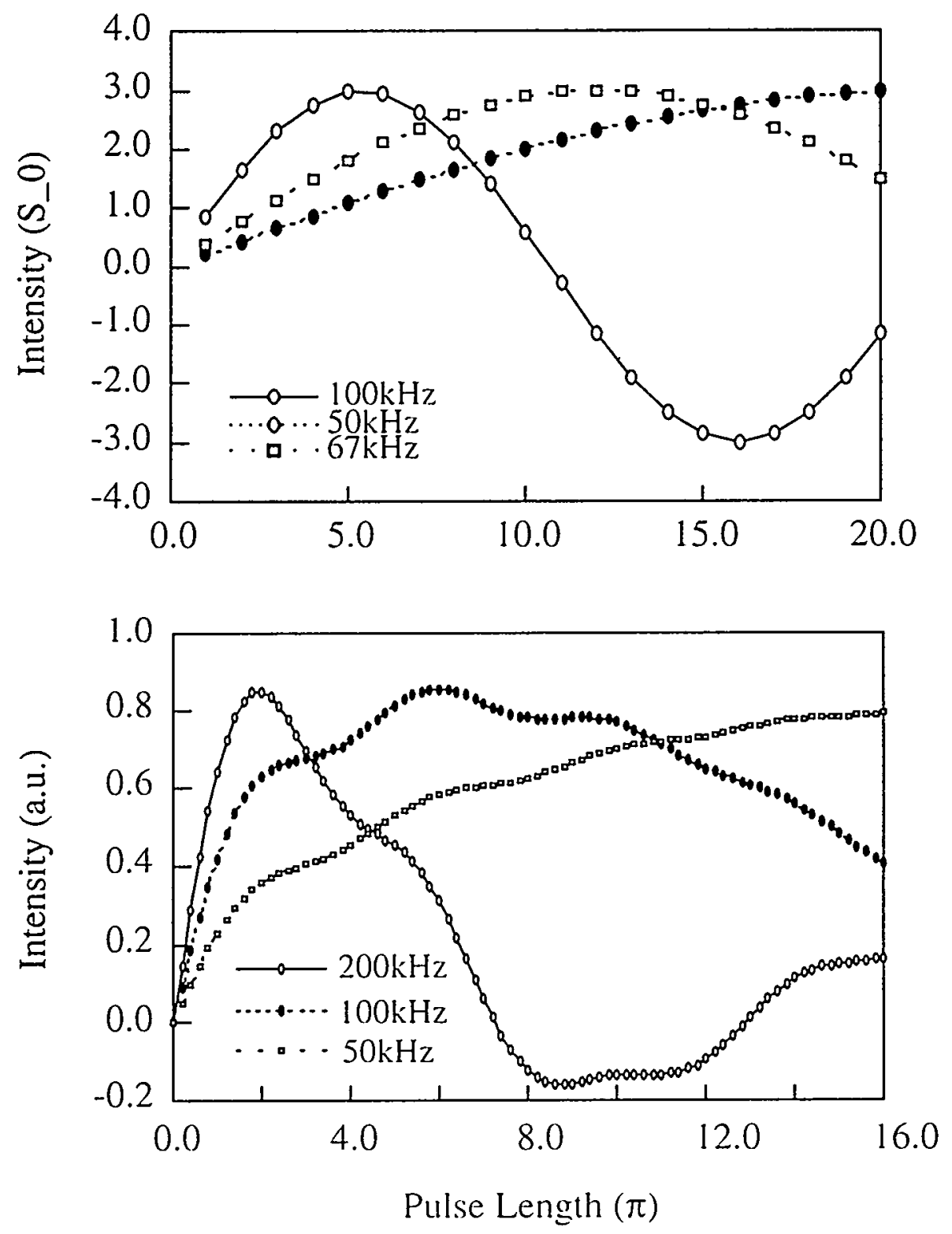

Figure 3.14: (Galculated triple-guanlun excitation eflicioncy for single crystal (upper) and powder (lower) samples as a function of pulse length. The magnitude of the RF field strenght $\left(\omega_{1}\right)$ is shown as Irgends. The calculation assumes that $\omega_{Q}=400 \mathrm{kH} \% . S_{0}$ is the maximum signal inlonsity for the single-quantum transition. 

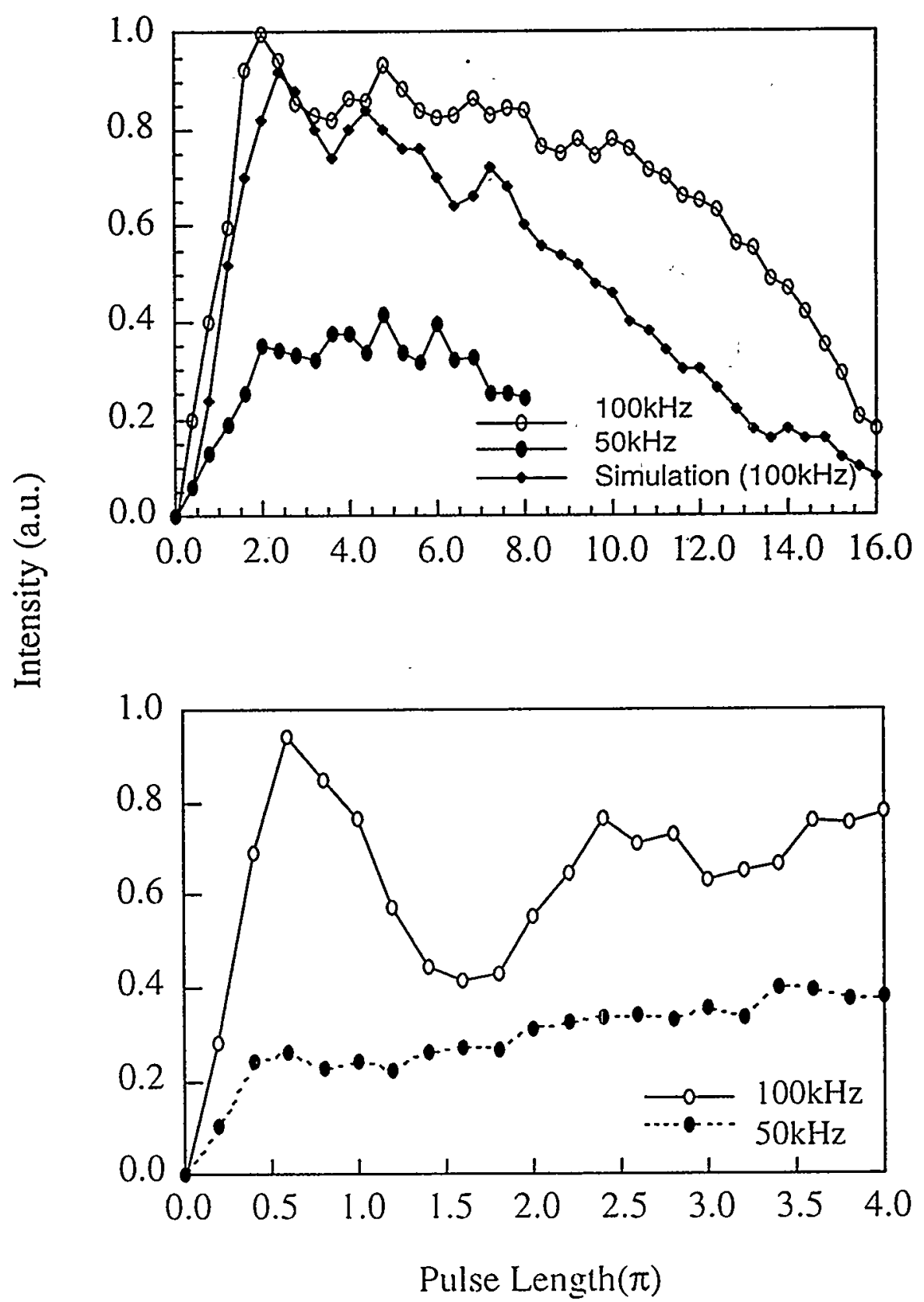

Figure 3.15: Experimental dal.a on triple-cuantum excitation (upper) and reconversion (lower) efficiency as a function of pulse length. The magnitude of the RF field strength $\left(\omega_{1}\right)$ is shown as legends. The calculation assumes thati $\omega_{Q}=400 \mathrm{kHz}$. Computer simulatied result is also shown in the upper graph. 
Unfortunately. $a_{x}^{23}$ quiclily drops to zero as the ralio between the quadrupolar frequency and the RF-pulse strength increasess. To sce this, notice that when $\omega_{1} \ll \omega_{Q}$,

$$
a_{x}^{23}<\sin ^{2} \theta \approx \tan ^{2} \theta=3\left(\frac{\omega_{1}}{\omega_{Q}}\right)^{2}
$$

This suggests that the reconversion efficiency is proportional to the square of the ratio between the RF field strength and the quadrupolar frequency, which is often very small. As a result, lhe reconversion problem is more serious than the excitation problem.

In Figure 3.16, the dependence of the reconversion efficiency on the reconversion pulse length is presented. Again, one secs the buildup of the single-cuantum signal in a short period of time $\left(\omega_{1} l=0.8 \pi\right)$. Howerer, the magnitude of the signal is very small. Neglecting spinning cffect in this case is appropriate since the pulse length takes only about $2 \%$ of a rolor cycle (Ser Figure 3.15$)$.

\subsubsection{Excitation and Reconversion: Computer Simulation}

To fully understand the excitation and reconversion processes under spinning condition, computer simulation has to be exploited $[8,66]$. This is because the Hamiltonians of the system al time $l$ and $t+\delta l$ do not commute to each other and the evolution of the density matrix has to be solved mumerically by calculating

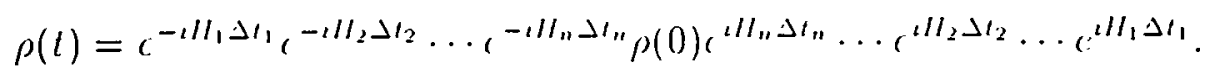

The calculation is often facilitated by the diagonalization of cach llamilionian in the equation [8]. 'The calculation on the excitation officiency is shown in Figure 3.15 (upper). The results cualitatively match those obtained by Amoureux [67] and Wu [68], that is, a relatively long pulse is neceled for excitation. 'The discrepancy may be due to the slightly diflerent paramerers we used in the simulation. The simulation 


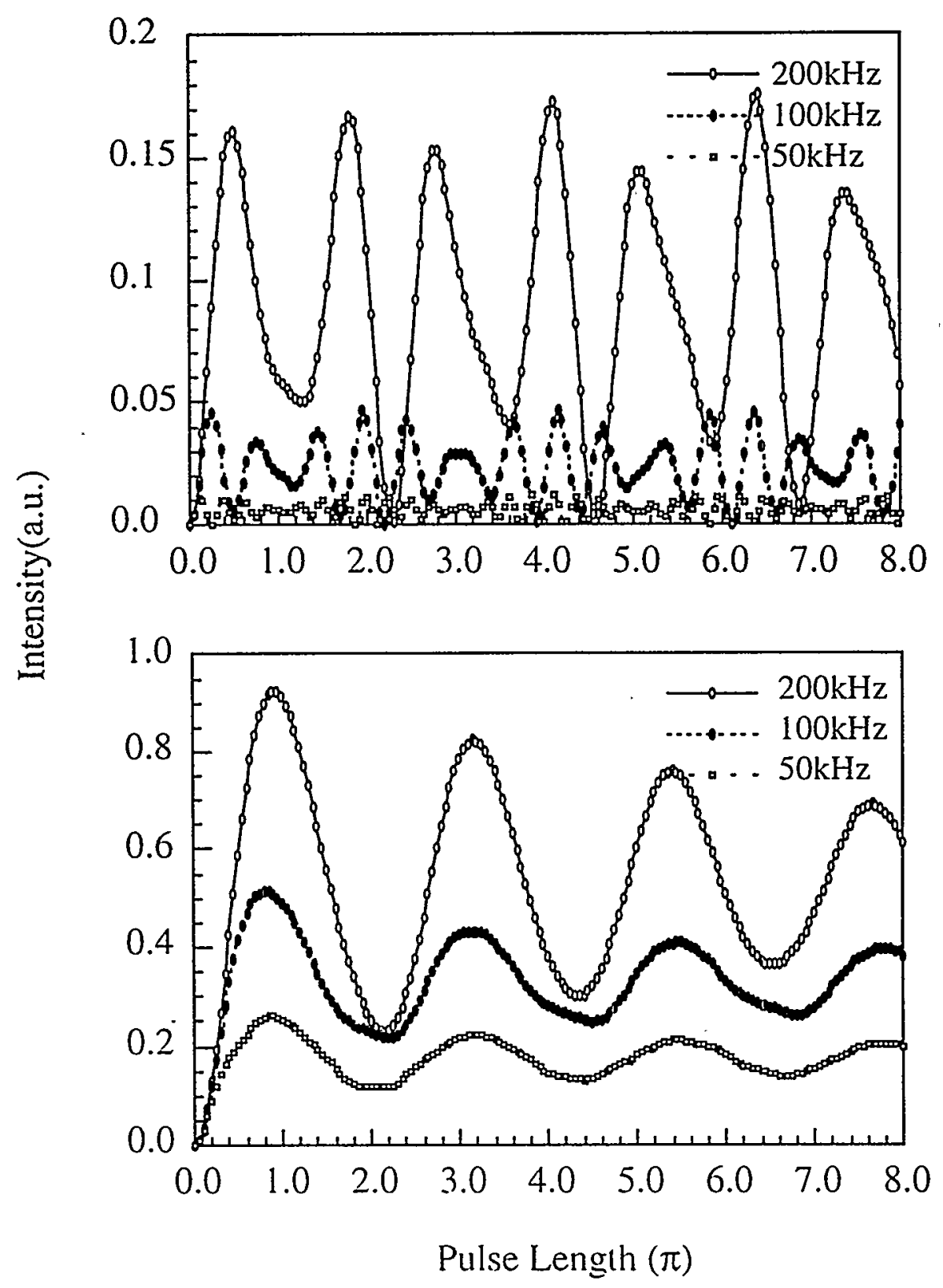

Figure 3.16: Calculated triplo-quantum to single-quantum reconversion efficiency for single crystal (upper) and powder (lower) samples as a. function of pulse length. The magnitude of the RF field strength $\left(\omega_{1}\right)$ is shown as legends. The calculation assumes that $\omega_{Q}=400 \mathrm{k}$ ilz. 


\begin{tabular}{ccccc}
\hline & \multicolumn{4}{c}{ Pulse length $(\mu \mathrm{s})$} \\
Exct./Recov. & $3 / 2$ & $5 / 2$ & $7 / 2$ & $9 / 2$ \\
\hline Excitation & $\frac{8}{3} \pi$ & $3 \pi$ & $\frac{8}{3} \pi$ & $\frac{5}{2} \pi$ \\
Reconversion & $\frac{2}{3} \pi$ & $\pi$ & $\pi$ & $\pi$ \\
\hline
\end{tabular}

Table 3.6: Pulse lengths that maximize the excitation and reconversion efficiency. also reproduces the short-term behavior of the experimental data. The interested readers should consult olher references $[67,6 \$]$. The best pulse lengths for excitation and reconversion are however, summarized in Table 3.6. Notice that the pulse lengths here are solid-state pulse lengths, and the result qualitatively agrees with the numbers obtained by the simple model which neglects the spinning effect.

\subsubsection{Excitation and Reconversion by Spin-Locking}

Another interesting mollool that is reported for excitation and reconversion was proposed by $W_{u}$ et al. [(i:3]. 'The pulse sequence and phase cycle are shown in Figure 3.17 and 'Table $3 . \bar{\imath}$, respectively. Instead of using a single long pulse for excitation, two pulses (without lime intoral in between) are used. The first pulse is a solid-state $90^{\circ}$ pulse, and hile scrond pulso is a spin-locking pulse phase shifted by $90^{\circ}$ with respect to the first pulse. The length of this second-pulse is set to $\tau_{r} / 4$, where $\tau_{r}$ is the rotor period. The reconversion pulse is a single pulse whose length is again set, to $\tau_{r} / 4$. This pulse is much longer than the optimal pulse length suggested by Amoureux [6it]. 'The efficiency of the secfuctice has been illustrated both by experiments and simulations. The most significanl advantage of the sequence is that it is more quanlilative.

The ley idea behind this now sequonce is that when the sample is spinning, the eigenstates of the spin IIamiltonian change with time. If initially the spin system is in $I_{X}^{23}$ state, part of the coherence is comverted into triple-quantum coherence $I_{X}^{14}$ at 


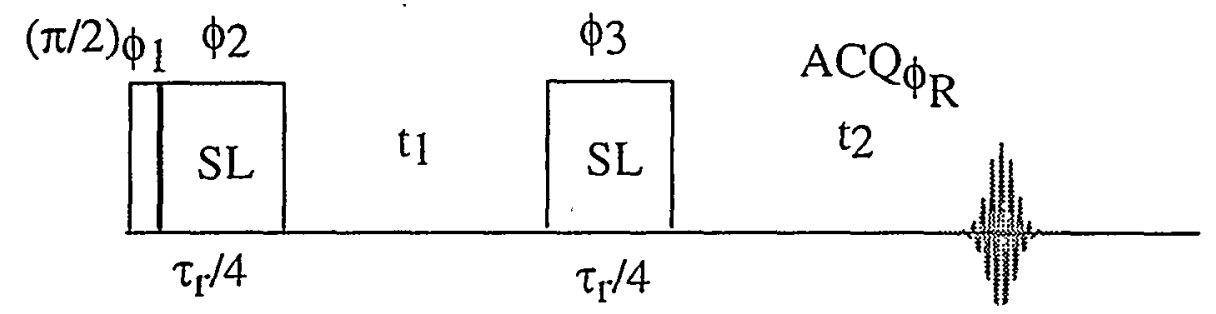

Figure 3.17: Spin-Locking pulse sequence for the excitation of triple-quantum coherence and its conversion back to singlc-quantum coherence.

\begin{tabular}{c|cccccc}
\hline \multicolumn{7}{c}{ Spin-Locking 3QMAS cosine } \\
\hline$\phi_{1}$ & $0^{\circ}$ & $60^{\circ}$ & $120^{\circ}$ & $180^{\circ}$ & $240^{\circ}$ & $300^{\circ}$ \\
$\phi_{2}$ & $90^{\circ}$ & $150^{\circ}$ & $210^{\circ}$ & $270^{\circ}$ & $330^{\circ}$ & $30^{\circ}$ \\
$\phi_{3}$ & $0^{\circ}$ & $0^{\circ}$ & $0^{\circ}$ & $0^{\circ}$ & $0^{\circ}$ & $0^{\circ}$ \\
$\phi_{r}$ & $0^{\circ}$ & $180^{\circ}$ & $0^{\circ}$ & $180^{\circ}$ & $0^{\circ}$ & $180^{\circ}$ \\
\hline \multicolumn{7}{c}{ spin-Locking 30.MAS sine } \\
\hline$\phi_{1}$ & $30^{\circ}$ & $90^{\circ}$ & $150^{\circ}$ & $210^{\circ}$ & $270^{\circ}$ & $330^{\circ}$ \\
$\phi_{2}$ & $120^{\circ}$ & $180^{\circ}$ & $2-10^{\circ}$ & $300^{\circ}$ & $0^{\circ}$ & $60^{\circ}$ \\
$\phi_{3}$ & $0^{\circ}$ & $0^{\circ}$ & $0^{\circ}$ & $0^{\circ}$ & $0^{\circ}$ & $0^{\circ}$ \\
$\phi_{r}$ & $0^{\circ}$ & $180^{\circ}$ & $0^{\circ}$ & $180^{\circ}$ & $0^{\circ}$ & $180^{\circ}$ \\
\hline
\end{tabular}

Table 3.7: 'The spin-locking $3 Q \mathrm{M} \Lambda \mathrm{S}$ phase cycle with redefinition of $t_{1}$ and $t_{2}$. 
$\tau_{r} / 2$ and returns to $l_{\lambda}^{23}$ after a full rotor cycle whon a spin-locking pulse is applied $[69,70,71]$. Also, part of the coherence is converted into $I_{X}^{14}$ at $\tau_{r} / 4$ and returns to $I_{X}^{23}$ at $\tau_{r} / 2$. If this is true, the first $90^{\circ}$ pulse in the sequence shown in Figure 3.17 creates coherence $I_{X^{\prime}}^{23}$, which evolves into $I_{X^{1}}^{1 \cdot 1}$ at $\tau_{r} / 4$. The third pulse, similarly transfer the coherence back into a single-quantum coherence.

Even though the authors have shown that their sequence is far more quantitative than the two-pulse sequence, the sequence has not been widely used untill now. One of the reasons may be that the sequence is only' efficient for spin- $\frac{3}{2}$ nuclei. Another reason is probably that, the secpucner is still not well-understood. According to Vega, the spin-locking elficiency for cuadrupolar nuclei is low and only under adiabatic conditions is the transfer of colrerence by spin-locking possible [70]. It seems controversial that the spin-locking secunence gives good results since the adiabatic condition is expected to be hard to fullill. People with further interests should read other references $[69,70,71]$.

\subsection{Comparison of DAS and MQMAS}

This section addresses sereral complomentary aspects of MQMAS and compares MQMAS with DAS [45]. Issues such as forsibility, linewidth, resolution are discussed. The chemical shift offect on the resultant spectra is then presented in great detail. It is also demonstrated here lhat ('S: 1 paramelers cau be extracted for quadrupoles using the mulliplo-qualutum strategr:

\subsubsection{Experiments}

Most of the spectra presentod in this chapter were obtained at.9.4T or $11.7 \mathrm{~T}$ using

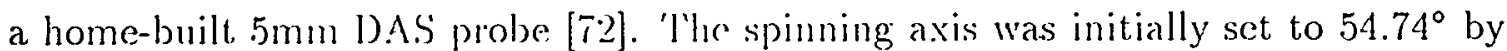
maximizing the observed sidcband intensity using $\mathrm{KB} B \mathrm{r}\left({ }^{\mathrm{s} 1} \mathrm{Br}\right)$ or deuterated HMB 
$\left({ }^{2} \mathrm{H}\right)$ as standards. The ${ }^{27} \mathrm{Ml}$ spectra were collected using a Doty Scientific $5 \mathrm{~mm}$ high-speed MAS probe [73]. Spinning speeds greater than $7.5 \mathrm{kH} \%$ were exploited in all experiments except for the one designed to give the intense $\mathrm{Na}_{2} \mathrm{C}_{2} \mathrm{O}_{4}$ sideband pattern. The RF power level in the DAS experiments was set to ensure selective excitation of the central transition, and a typical $90^{\circ}$ pulse length was about $7 \mu$ s. In the MQMAS experiments, high power $\left(35-40 \mathrm{kHz}\right.$ [or ${ }^{17} \mathrm{O}, 50-60 \mathrm{kHz}$ for other nuclei) was used to achieve efficient. excitation.

The shifted-echo DAS sequence $\left(\frac{\pi}{2}-t_{1}-\frac{\pi}{2}-\right.$ hop $-\frac{\pi}{2}-\tau_{e}-\pi-$ acq. $)$ [50] and shifted-echo MQMAS [51] sequence used in our experiments were described in Section 3.3. The echoes were ty'pically shifted out by twenty to thirty rotor cycles (many milliseconds if possible) to achicve high sensitivity and undistorted signal. If the sensitivity was a problem due to $\mathrm{T}_{2}$ relaxation during echo formation, the simple two-pulse MQMAS seçuence (Figure 3.12) was used. $540^{\circ}$ solid-state pulses were applied for the excitation and reconversion, which according to the results of Amoureux [67], do not give the maximum multiple-quantum excitation and reconversion efficiency. The data were processed according to the method suggested by Grandinetti $[50,51]$. RMN software was used and can be downloaded from the following URL: http://www.chemistry.ohio-stal.c.cdu/grandinelti/RNN/rmn.html.

All of the inorganic compounds used in the experiment, were obtained from commercial sources, lypically with a slated purity of at least $98 \%$. The anorthite and ${ }^{17} \mathrm{O}$ enriched stilbite wore mado at Stanford university and characterized by X-ray diffraction (XRD) and ${ }^{2 \%}$ Si N.MR.

\subsubsection{Feasibility}

MQMAS spectra of ${ }^{23} \mathrm{Na}\left(\mathrm{I}=\frac{3}{2}\right),{ }^{8 T} \mathrm{Rb}\left(\mathrm{I}=\frac{3}{2}\right),{ }^{2 \pi} \mathrm{Nl}\left(\mathrm{I}=\frac{5}{2}\right),{ }^{17} \mathrm{O}\left(\mathrm{I}=\frac{5}{2}\right)$ and ${ }^{45} \mathrm{Sc}\left(\mathrm{I}=\frac{7}{2}\right)$ in some model compounds are slown in Figure 3.18 . The typical recycle delay varies 
between 0.5 and 1 second for different samples, resulting in an acquisition time no longer than 12 hours. This suggests that MQMAS spectrum can be acquired in a reasonable time period for these nuclei (also for ${ }^{11} \mathrm{~B}, \mathrm{I}=\frac{3}{2}[62]$ ) when the $\mathrm{T}_{1}$ of the sample is not very long. The quadrupolar coupling constants $\left(C_{Q}\right)$ are $3.6 \mathrm{MHz}$ for ${ }^{87} \mathrm{Rb}$ in $\mathrm{RbClO}_{4}$ [24], 2.4 $\mathrm{MHz}$ for ${ }^{23} \mathrm{Na}$ in $\mathrm{Na}_{2} \mathrm{C}_{2} \mathrm{O}_{4}$ [58], about. $5 \mathrm{MHz}$ for the ${ }^{45} \mathrm{Sc}$ sites in $\mathrm{Li}_{3} \mathrm{Sc}_{2}\left(\mathrm{PO}_{4}\right)_{3}, 2.8-8.4 \mathrm{MII} \%$ for ${ }^{27} \mathrm{Al}$ siles in anorthite [60] and $3.4 \mathrm{MHz}$ and $4.8 \mathrm{MHz}$ for the two types of oxygen sites in stilbite. The MAS dimension of these spectra usually show woll-dofinced MAS powder patterns, suggesting a relatively even excitation of the triple-(quantum colucronce.

The single-hop DAS spect.ra for the above ${ }^{8 \pi} \mathrm{Rb} .{ }^{23} \mathrm{Na}$ and ${ }^{17} \mathrm{O}$ samples were also recorded. However, accunisition of the ${ }^{11} \mathrm{~B},{ }^{2 \pi} \mathrm{Al}$ and ${ }^{45} \mathrm{Sc}$ DAS spectra was far less successful because of the short $T_{1}$. Typically, 30-50 milliscconds are necded to flip the spinner axis in a DAS experiment which means it can only be applied to samples with $\mathrm{T}_{1}$ longer than 100-150ms. Another limiting factor for DAS is the homonuclear dipolar coupling, which creates colerences that may not be restored following axis reorientation [42]. There is 110 stlch limitation for MQQMIAS because a hopping period is not necessary betwern the two correlated crolution periods.

The major limiting factor for MQMAS' is the magnitude of the quadrupolar interaction. Since the triph-equantum transition is forbidelen to Ist-order, the MQMAS

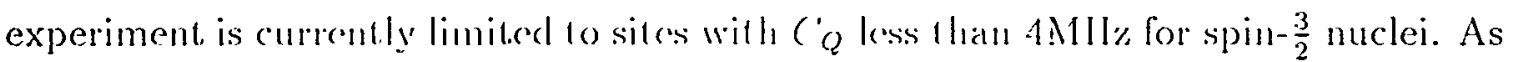

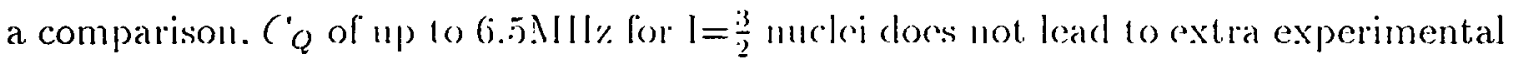
difficulties for D.AS [21]. In Hoe extreme, Massiot e1 al. have obtained a ${ }^{71} \mathrm{Ga}\left(\mathrm{I}=\frac{3}{2}\right)$ DAS spectrum of $3-\left(\mathrm{Cia}_{2}()_{3}\right.$ will a ("Q of $12 . \mathrm{Mll} \%[\mathrm{~T} \cdot 1]$.

As shown earlier in lhis chapter. lhe excitation clficiency of the triple-quantum coherence is determined by $\frac{w_{S}}{w_{1}}$. To achieve maximum excitation efficiency, highest power level is usually used. Whom tho Rl strongth is fixed, the quantity that de- 

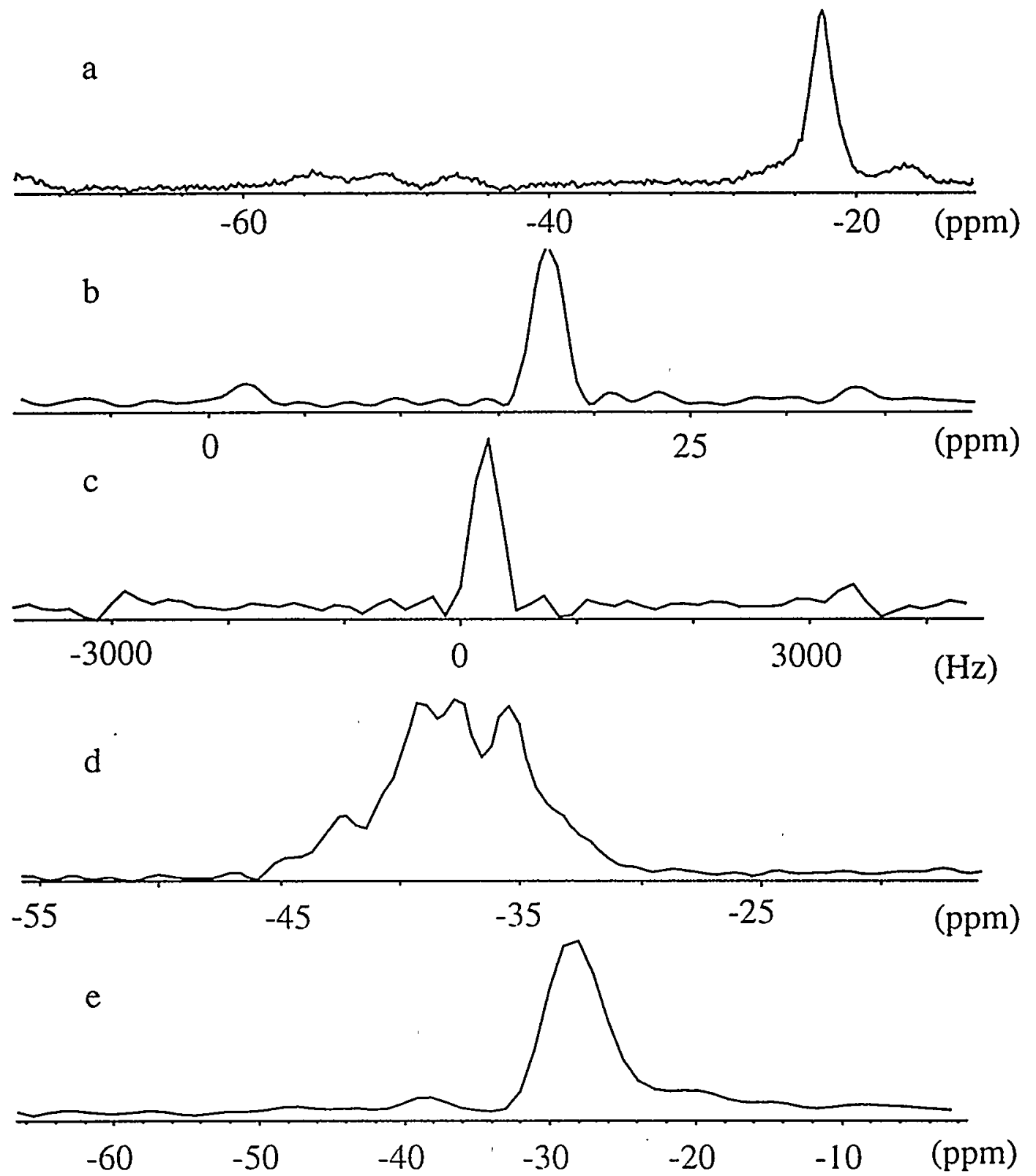

Frequency

Figure 3.18: 3QMAS spectra of (a) ${ }^{87} \mathrm{RbC}^{\prime} \mathrm{O}_{4}$ (b) ${ }^{23} \mathrm{Na}_{2} \mathrm{C}_{2} \mathrm{O}_{4}$ (c) ${ }^{45} \mathrm{Sc}$ in $\mathrm{Li}_{3} \mathrm{Sc}_{2}\left(\mathrm{PO}_{4}\right)_{3}$ (d) ${ }^{27} \mathrm{Al}$ in anorthite (e) ${ }^{17} \mathrm{O}$ in stilbite. Spectra (a), (b), (d) were taken at $11.7 \mathrm{~T}$ while the other two were taken at 9.4T. Frequencies are referenced to $1 \mathrm{M} \mathrm{RbNO}_{3}$ for ${ }^{8 \pi} \mathrm{Rb}, 1 \mathrm{M} \mathrm{NaCl} \mathrm{for}{ }^{23} \mathrm{Na}, 1 \mathrm{MAl}\left(\mathrm{NO}_{3}\right)_{3}$ for ${ }^{27} \mathrm{Al}$ and $\mathrm{H}_{2} \mathrm{O}$ for ${ }^{17} \mathrm{O}$. Spectrum (d) is a slice takcol along the isotropic dimension, which has better resolution than the isotropic projection. 
termines the excitation efficiency is $\omega_{\varphi}$ which is clefined as

$$
\omega_{Q}=\frac{C_{Q}^{\prime}}{2 I(2 I-1)}
$$

and smaller $\omega_{Q}$ usually means better excitation unless $\omega_{Q}$ is vanishingly small. It is thus not surprising to see the officient excitation for ${ }^{2 \pi} \mathrm{Al}$ (Figure 3.1Sd, anorthite, with $C_{Q}$ up to $8.4 \mathrm{MHz}$ ), but the inferior excitation for ${ }^{87} \mathrm{Rb}$ (Figure $3.18 \mathrm{Sa}, \mathrm{RbClO}_{4}$, $C_{Q}=3.6 \mathrm{MHz}$ ). The smaller scaling factor for $1>\frac{3}{2}$ nuclei suggests that the observation of $C_{Q}$ up to $10 \mathrm{MHz}$ for ${ }^{2 \pi} \mathrm{Al}$ is feasible, based on the relatively efficient excitation of the triple-quantum coherence for ${ }^{23} \mathrm{Na}$ (e.g. $\mathrm{Na}_{2} \mathrm{C}_{2} \mathrm{O}_{4}, \mathrm{I}=\frac{3}{2}, \mathrm{C}_{Q}=2.4 \mathrm{MHz}$ ). This is actually demonstialled by our experiment on liyanite (see next chapter), which contains ${ }^{27} \mathrm{Al}$ sile with $C_{Q}^{\prime}$ up to 10.0MIll\%. The fact that the single pulse excitation scheme is relatively efficiont for spin- $\frac{5}{2}$ muclei is important because $C_{Q}$ for ${ }^{27} \mathrm{Al}$ and ${ }^{17} \mathrm{O}$ (both spin- $\frac{5}{2}$ ) are smallor than $10 \mathrm{MH} \%$ in a lot of commonly used technological materials. However, care must be taken in the use of this techmique for the study of site quantification.

\subsubsection{Linewidth}

The DAS and MQQMAS linewidh (full widllı al half maximum, FWIIM) of some model compounds are compared in Tahlo :3.S. Also shown in this table is the linewidth data from MAS and Hahu-celes experimonts ('l'-limewidh). In addition, the DAS

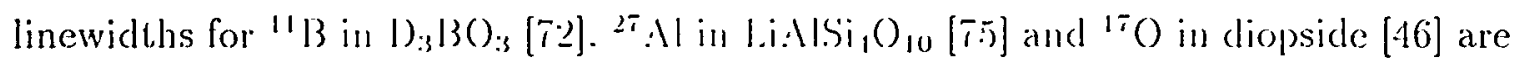
also included.

The DAS linewidth increases from alone $200 \mathrm{~Hz}$ for ${ }^{87} \mathrm{Rb}$ to $1.2 \mathrm{kHz}$ for ${ }^{27} \mathrm{Al}$, consistent wilh the increasing homonuclear dipolar coupling strength. The MQMAS linewidth, on the ofluer land, is largely nucleus-independent, spanning the range

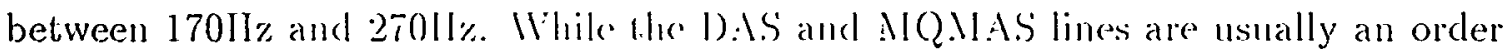




\begin{tabular}{l|cccc}
\hline & \multicolumn{4}{|c}{ Linewidth(Hz) } \\
\cline { 2 - 5 } Nucleus/compound & DAS & MQMAS & Hahn-Echo & MAS \\
\hline${ }^{87} \mathrm{RbNO}_{3}$ & 160 & 180 & 10 & 2500 \\
${ }^{87} \mathrm{RbClO}_{4}$ & 250 & 270 & 10 & 5000 \\
${ }^{23} \mathrm{Na}_{2} \mathrm{C}_{2} \mathrm{O}_{4}$ & 500 & 260 & 25 & 3500 \\
$\mathrm{D}_{3}{ }^{11} \mathrm{BO}_{3}[72]$ & 1100 & - & - & 4500 \\
${ }^{27} \mathrm{Al} \mathrm{Kyanite}[60]$ & - & 170 & 30 & 7300 \\
${ }^{27} \mathrm{Al} \mathrm{LiAlSi}{ }_{4} \mathrm{O}_{10}[76]$ & 1200 & - & - & 4200 \\
${ }^{17} \mathrm{O}$ Diopside [75] & 120 & 100 & - & 3800 \\
${ }^{17} \mathrm{O}$ Stilbite & 600 & 270 & 30 & 4500 \\
\hline
\end{tabular}

Table 3.8: Comparison of the DAS, MQMAS, echo and MAS linewidths for some model compounds.

of magnitude narrower than the MAS lines, they are an order of magnitude broader than the echo-linewidth. This is not a surprise, since magnetic field inhomogeneity as well as inhomogeneous broadenings is refocused by an echo seguence, leaving only the intrinsic $\mathrm{T}_{2}$ relaxation under MAS. To understand completely why the MQMAS lines are so much wider than the echo lines requires more detailed and careful measurements to evaluate the contribution of each broadening mechanism. The residue dipolar coupling, the defects in the crystal and the ficld inhomogeneity can all be the dominating broadening mechanism.

The DAS linewidth can be related to the magnitude of the homonuclear dipolar interaction in the system [42]. The bilinear terms in the density matrix arising from the dipolar Hamiltonian can not be retained fhrough a. DAS angle change. Thus the dipolar Hamiltonian acts purcly as a relaxation superoperator and it is not refocused at the DAS echo maximum. The independence of the MQMAS linewidth on the nucleus of interest at: least suggests that homonuclear dipolar coupling is not a crucial factor for the implementation of the MQMAS experiment, which is a major advantage of MQMAS over DAS.

Strong heteronuclear dipolar coupling may render the MQNAS spectra broad 
too. The problem secms scrious only' when '/l is involved and can be overcome by ${ }^{1} \mathrm{H}$ decoupling [7T].

\subsubsection{Resolution}

As shown in Eq̣n 3.31, the observed MQMAS shift.s are scaled by two factors $k_{1}$ and $k_{2}$ and different from DAS $\left(k_{1}=k_{2}=1\right)$ shifts. This makes the resolution of a MQMAS spectrum dependent not only on $\delta_{i s o}$ and $w_{i s v}^{2 Q}$, but also on $k_{1}$ and $k_{2}$. Some general conclusions about resolution can be drawn, but for a specific sample, care must be taken in spoctral interpretation.

Eqn 3.34 indicates that two chemically different sites having the same quadrupolar coupling constant will have a firequency separation proportional to $k_{1}$ in the MQMAS spectrum. If $\left|k_{1}\right|$ is greater than 1.0 , bettor resolution is expected for MQMAS; on the other hand, if $\left|k_{1}\right|$ is smaller than 1.0. DAS las bet tor resolution. The factor $k_{2}$ has a similar effect, on resolution, when the chemical shift of the two sites is the same. In the case that $I=\frac{3}{2}$, MQMAS has better resolution than DAS according to Table 3.1 , and the opposite is truc for $I>\frac{3}{2}$ unclci. For $I=\frac{3}{2}$ nuclei, the enhanced resolution of MQMAS over DAS is radily sorn in Figures 3.9) and 3.11 where the separation of the two MQMAS peaks in RI, $\mathrm{NO}_{3}(9 \mathrm{ppm})$ is twice lhat in the DAS spectrum (5ppm). For $I=\frac{5}{2}$ nuclei, an (xample is given in Figure 3. I9). Which is an ${ }^{17} \mathrm{O}$ stilbite spectrum taken at 9.4T. In this (asce. DAS gives 1 wo pattially resolved peaks, whereas only one peak is observed in the MQQNAS spectrum. Similar resolution limitation was also $\sec$ for ${ }^{45} \operatorname{Sic}\left(I=\frac{7}{2}\right)$.

Despite the small scaling factors $\left(h_{1}=-17 / 31 . h_{2}=10 / 31\right)$ for spin- $\frac{5}{2}$ nuclei, ${ }^{27} \mathrm{Al}$ is an ideal muckeus for MQMAS. as will be demonstraled in the next chapter [60]. MAS spectia for ${ }^{27}$ Al are nsially too broad to take advantage of the improved spectral dispersion. 'The morkanc conpling constants for ${ }^{2 \top} \Lambda$ in most materials [7S] 


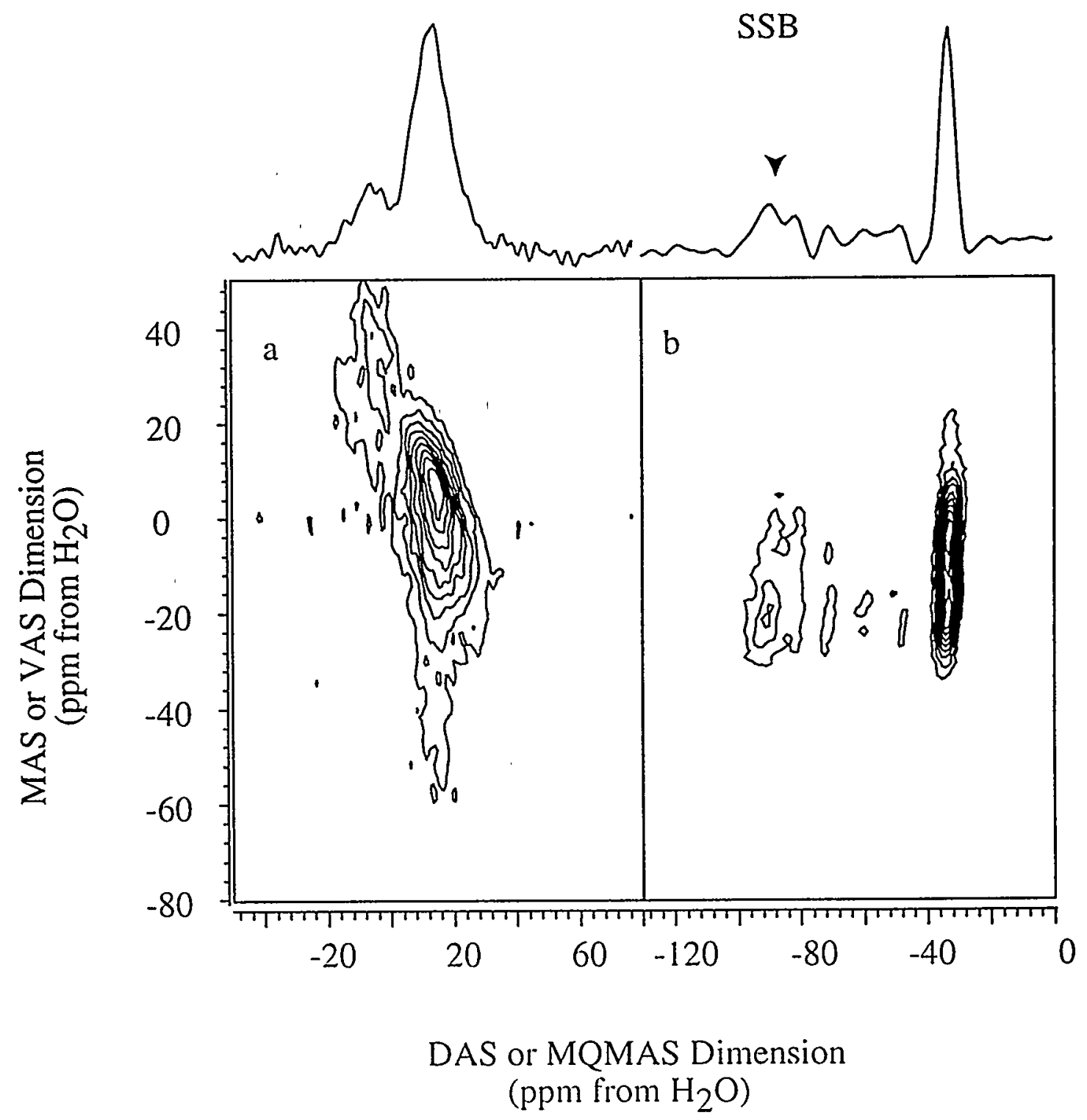

Figure 3.19: 9.4T DAS (a) and MQMAS' (b) spectra of ${ }^{17} \mathrm{O}$ labeled stilbite. Two DAS peaks are discernible, but only one peak is obscrved in the MQMAS spectrum (the left hand side intensily is from spectral distortion and sideband). 
make it very suitable for MQMAS studies. In adclition, the decreased resolution in the 3QMAS spectrum can be overcome by correlating the quintuplc-quantum coherence with the single-quantum colierence [79].

\subsubsection{Chemical Shift Effect}

It is somewhat interesting to sce that two of the three rubidium sites in ${ }^{87} \mathrm{RbNO}_{3}$ collapse together in the 11.TT MQMAS spectrum. At the same ficld strength, three peaks exist in the DAS spectrum even though the MQMAS spectrum is expected to have better resolution. 'This problem happens also in the RbNO $\mathrm{O}_{3}$ DAS spectrum at 7.0T [24], whereas at that field strength, the MQNAS spectrum has three wellresolved peaks [51]. 'This overlap indicates that sites with different chemical shifts and quadrupolar intoractions call appear at the same frequency in a DAS or MQMAS spectrum. Such an accidcontal overlap) is a result of the cancellation of the chemical shift difference and the 2nd-order cularlrupolar shift difference.

For well-crystallized samples, the accidental overlap can be overcome by multiplefield experiments. Onc can also perform bollh DAS and MQNAS experiments on the same sample at only one fichl since the overlap conditions are different for these two experiments. In this respere. the combination of D.AS and MQMAS removes the possibility of spect wal misinterpretation.

For matcrials with a contimnous distribution of chemical shifts and quadrupolar coupling constants (as in man! amorplious and glassy matcrials), the problem of overlapping peaks limits (hr applicalility of I):SS and MQQMAS. 'To sce this, I will give a brief description of the gonchal fatures of the ${ }^{17} \mathrm{O}$ 1) ASs spectra for silicate glasses [80, 81]. D).S spertra of these materials are broadened usually from one to twenty kilohertz by the distribution of chemical sites (which have a distribution of chemical shift and quadrupolar parameders). Because DAS gives a two-dimensional 
spectrum correlating the isolropic DAS shifts and the anisotropic interactions, an anisotropic slice taken perpendicular to the DAS dimension corresponds to a VAS spectrum and can be simulatied to provide a set of quadrupolar $\left(C_{Q}\right.$ and $\left.\eta_{Q}\right)$ and chemical shift $\left(\delta_{i s o}\right)$ paramcters (See Figure 3.9). These parameters are related to structural information such as the Si-O-Si bond angles for bridging oxygen in ${ }^{17} \mathrm{O}$ glass (Eqn 4.1). The above approach assumes that each slice that is taken out corresponds to only one type of site. Because it is possible that sites with different chemical environments show up at the same frequency; this assumption does not necessarily hold.

As an example, consider lhe amorphous $\mathrm{Si}^{17} \mathrm{O}_{2} \mathrm{DAS}$ datia obtained by Baltisberger et al. [82] Using the quadrupolar and chemical shift parameters they obtained, the isotropic shifts in DAS, triple-quantum MAS and quintuple-quantum MAS spectra were calculated using Eqn 3.2:3 and 3.31. The results are shown in Figure 3.20a, where $100 \mathrm{ppm}$ is first subtracted from the 5QMAS sbifts and then plotted. The data points on the left side of the figure are characterized by substantial error bars, which is mainly a result of simulat ion errors. The observed DAS shifts increase monotonically as a function of the slice number whereas the multiple-quantum shifts do not vary monotonically over the corresponding slices. What is also noted is that the spread, of MQMAS shifts is much smallor for the same siles as in DAS as a result of the scaling factors in Ecn 3.34. For many of the ${ }^{17} \mathrm{O}$ inorganic glasses, the quadrupolar coupling constant.s $\left(C_{Q}{ }_{Q}\right)$ decresse with the increase of the isotropic chemical shifts over a wide range of the Si-O-Si bond angles [82, 81]. The effect of a decreasing $C_{Q}$ is to shift the MQWAS spredra to higher frepuency (more positive ppm values). This effect is partially undonc by the simullancous increase of the isotropic chemical shift (note that $k_{1}<0$ ). The not result is a smaller spread of shift.s in MQMAS spectra of ${ }^{17} \mathrm{O}$ glasses than onc would gow. from a similar DAS experiment. The reason for the 
difference between DAS and MQMAS' is that. $k_{1}$ and $k_{2}$ in DAS have the same sign (both plus), but they have opposite signs in MQQMAS.

While Figure 3.20a shows that glassy samples may have a potential problem with overlap, it is worth considering a crystalline ${ }^{17} O$ sample where $C_{Q}$ and $\eta_{Q}$ are better defined. Pigure 3.20lo shows similar calculations for the five ${ }^{17} \mathrm{O}$ sites in wellcrystallized cocsite [47]. All five sites are clearly separated in DAS', but sites 3,4 and 5 are expected to overlap in the 3QMAS spectrum, and sites 4 and 5 are expected to overlap in the 5QM $\mathrm{QS}$ spectrum. This sort of overlap is possible in any sample depending on the relative sizes of the coupling constants and shifts. In this regard, performing all thre experiment.s (I).SS, 3QMAS and 5QMAS) would provide useful overall information as the overlap conditions for these techniques are different.

\subsubsection{Chemical Shift Anisotropy}

As discussed in the theory part of MQQMAS, the CSA effect is magnified by a factor of $2 m$ for the $m \leftrightarrow-m$ transition. which allows it to dominate the triple-quantum spectrum when its magnitude is comparable to that of the end-order quadrupolar interaction in a single-cluant.mm sperelrum. In this case, anisot ropic 2nd-order quadrupolar effect, may be neglected in the triple-cuantum dimension. Simulation of the

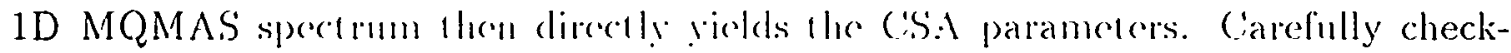
ing Table 2.3 suggests that lhis approach is only ralid for $I=\frac{3}{2}$ muclei (as long as 3QMAS is involved). IThen $/ \neq \frac{3}{2}$. lhe 2mel-order cuadrupolar affect is also amplified. However, when $I=\frac{3}{2}$ the magnitude of the 2nd-order quadrupolar interaction for the triple-quantum coherence is scaled down (by a firctor of $\frac{7}{9}$ ), compared to that for the single-quantum colurence.

Figure 3.21 is the onc- and nwo-dimensional $11.7 \mathrm{~T}{ }^{87} \mathrm{Rb}$ MQMAS $\left(\omega_{r}=8.9 \mathrm{kHz}\right)$ spectra of $\mathrm{Rb}_{2} \mathrm{CrO}_{4}$. T'wo sites exist in this componnd, but only the one with smaller 

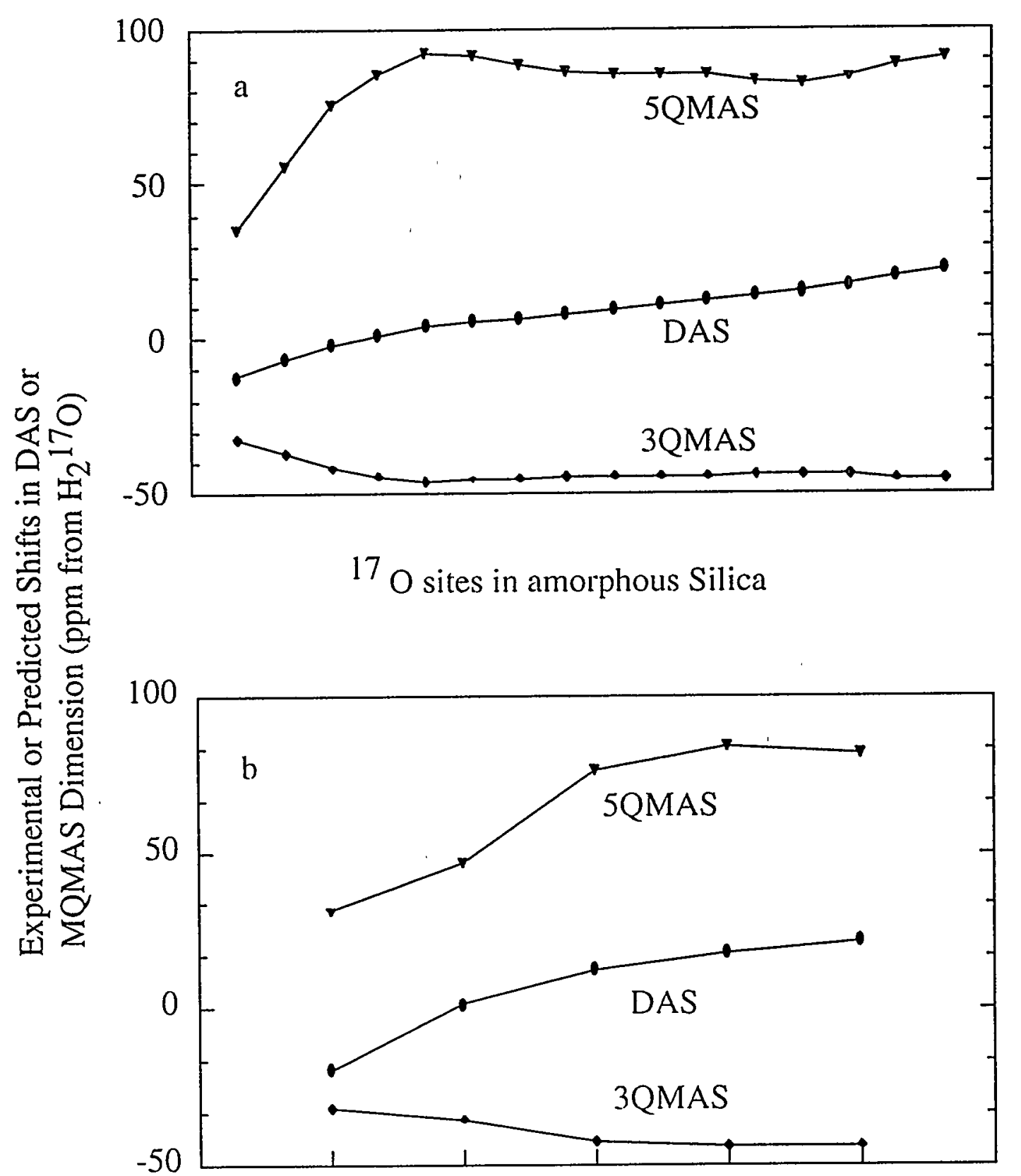

${ }^{17} \mathrm{O}$ sites in Coesite

Figure 3.20: Comparison of the resonance frequencies of DAS, 3QMAS and 5QMAS for amorphous $\mathrm{Si}^{17} \mathrm{O}_{2}$ (a) and coesite (b). The Latronor frequency is $67.7 \mathrm{MHz}$ corresponding to ${ }^{17} \mathrm{O}$ a.l. $11.7 \mathrm{~T}$. T'he isolropic chomical shift and quadrupolar coupling constant values are taken from [82] for $\mathrm{SiO}_{2}$ glass, and from [47] for coesite. $100 \mathrm{ppm}$ is subtracted from the 5QMIAS shifts and then ploted in the figure. 


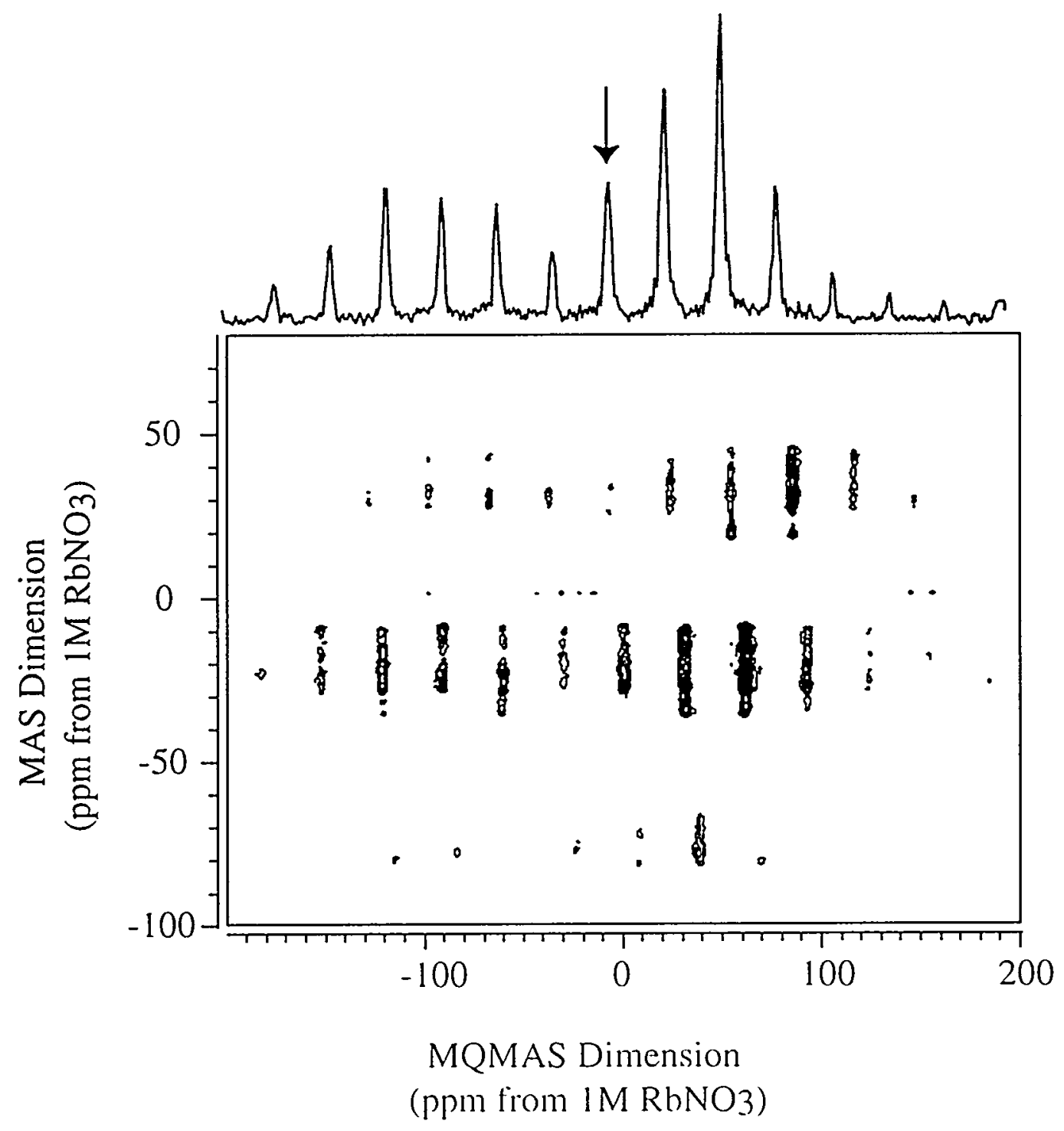

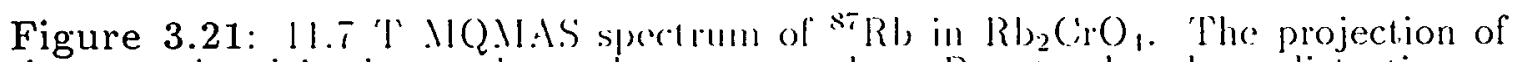
the centerband is shown above the contom plot. Due to the phase distortion, a magnitude spectrum is shown and the arow points to the centerband. The simple two-pulse sequence is used. 


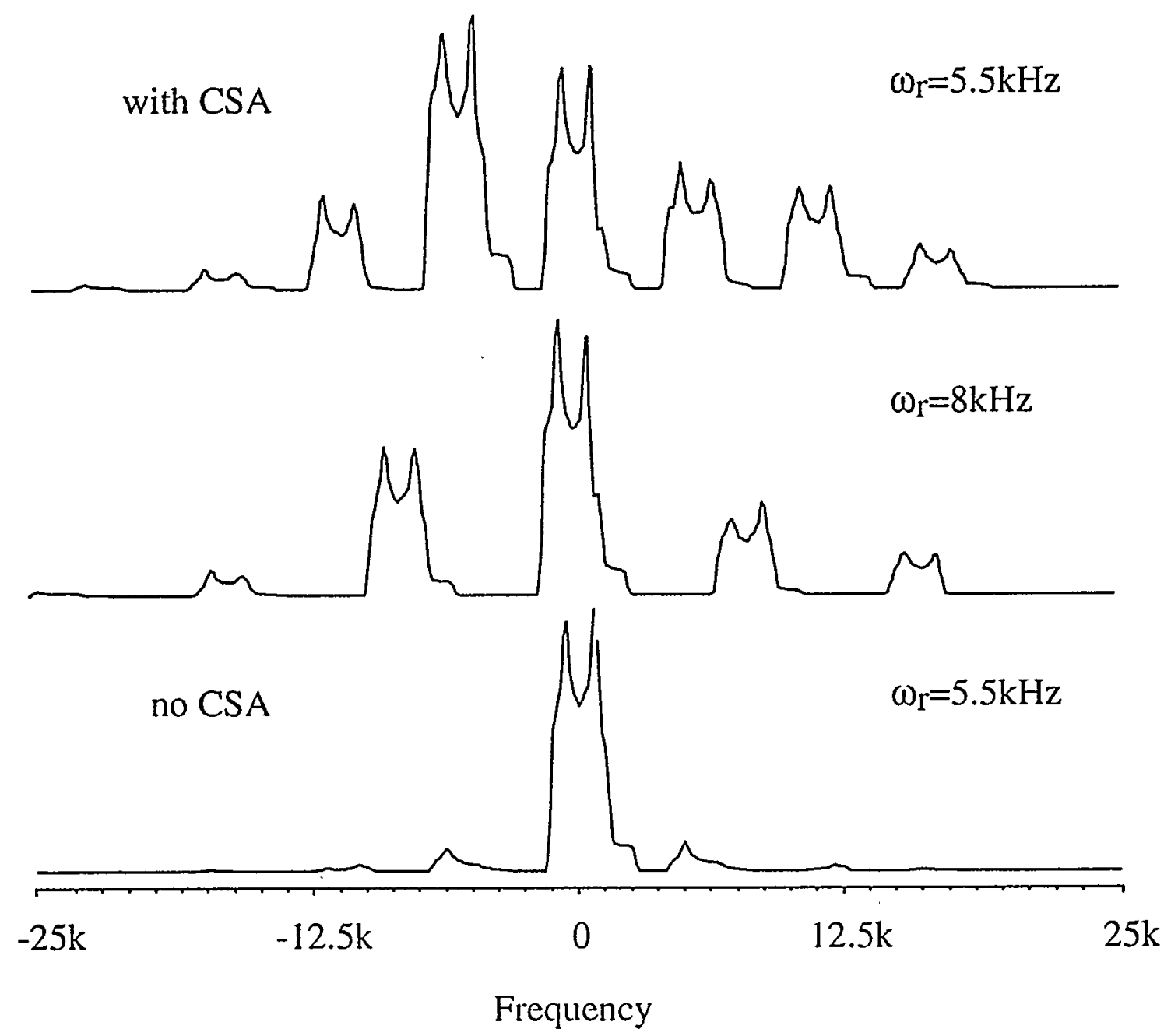

Figure 3.22: Simulated II.7T MAS spectrum of ${ }^{8 \tau} \mathrm{Rb}$ in $\mathrm{Rb}_{2} \mathrm{CrO}_{4}$. Experimental spectrum (not. shown) can not be reproduced if (SA is neglected.

$C_{Q}$ is observed. The spectrum has a lot of sidebands $\left(\frac{m k+n}{1+k} \omega_{r}, n \omega_{r}\right)$ away from the centerband in the wo-dinensional frepuency space. conlirming the conclusions on the MQMAS sideband pattern [15. \$:3]. The simulated MAS spectra that either considers, or does not consider, the chemical shift anisotropy are shown in Figure 3.22.. It is clear that chemical shift anisolropy plays an important role in interpreting the data.

Figure 3.23 shows and compares the ID DAS and NQMAS spectra of ${ }^{87} \mathrm{Rb}$ in $\mathrm{Rb}_{2} \mathrm{CrO}_{4}$. The MQMAS projection shows well-defined sideband pattern that is sim- 
ulated in Figure 3.23c. The C'SA and the end-order quadrupolar broadening for $\mathrm{Rb}_{2} \mathrm{CrO}_{4}$ at $11.7 \mathrm{~T}$ are $27 \mathrm{kll} \%\left(\delta_{C: s}=-110 \mathrm{ppm}\right)$ and $13 \mathrm{kHz}\left(\mathrm{C}_{Q}=3.5 \mathrm{MHz}\right)$, respectively [\$4]. Since they are somewhat comparable, the combined effect of CSA and quadrupolar interaction is complicalled, and the DAS and MAS spectra are not sensitive to variations in l.Ire (SSA parameters. It is then very difficult to obtain CSA information from either the DAS or MAS spectra. However, as CSA is amplified to $75 \mathrm{kHz}$ in the MQMAS dimension, the MQMAS spectrum of $\mathrm{Rb}_{2} \mathrm{CrO}_{4}$ covers a much larger frequency range and neglecting the 2nd-order quadrupolar interaction in simulating the MQMAS sideband intensities is possible. From Figure 3.23c, the magnitude and asymmetry parameter of CSA are determined ( $\delta_{C S}=-110 \mathrm{ppm}$, $\left.\eta_{c s}=0.0\right)$. These values are in good agreement with those determined by a switchingangle spinning (SAS) approach [ 81$]$ (chapter 6) but do not agree with other numbers found in the literature [85]. The $\mathrm{Rb}_{2}\left(\mathrm{C} \cdot \mathrm{O}_{1}\right.$ spectrum here also serves as an example to demonstrate that relatively large ('SA does not limit the implementation of MQMAS.

\subsection{Multiple-Quantum Variable-Angle Spinning}

The concept of mulliplr-guantum NAIR can be extended to the case that the sample is spinning at angles ofler than the magic-angle. To sec how this can lead to other information not acessible 1 lorough MQQM:LS, consider a spin- $\frac{3}{2}$ nucleus spinning at $70.12^{\circ}$ or $30.56^{\circ}$, where $P_{1}(\cos ())=0$. Since $\left({ }_{1}(3 / 2,3 / 2)=0\right.$ ('lable 2.3$)$, Ecun 2.83 reduces to

$$
\omega_{3 / 2 \leftrightarrow-3 / 2}^{2 Q}=C_{0}\left(\frac{3}{2}, \frac{3}{2}\right) \omega_{t s \omega}^{2 Q}
$$

This means that anisotropic Ind-order cuadrupolar interaction does not broaden the triple-cuantum spect rum. The spect rum is however. not a high-resolution spectrum 

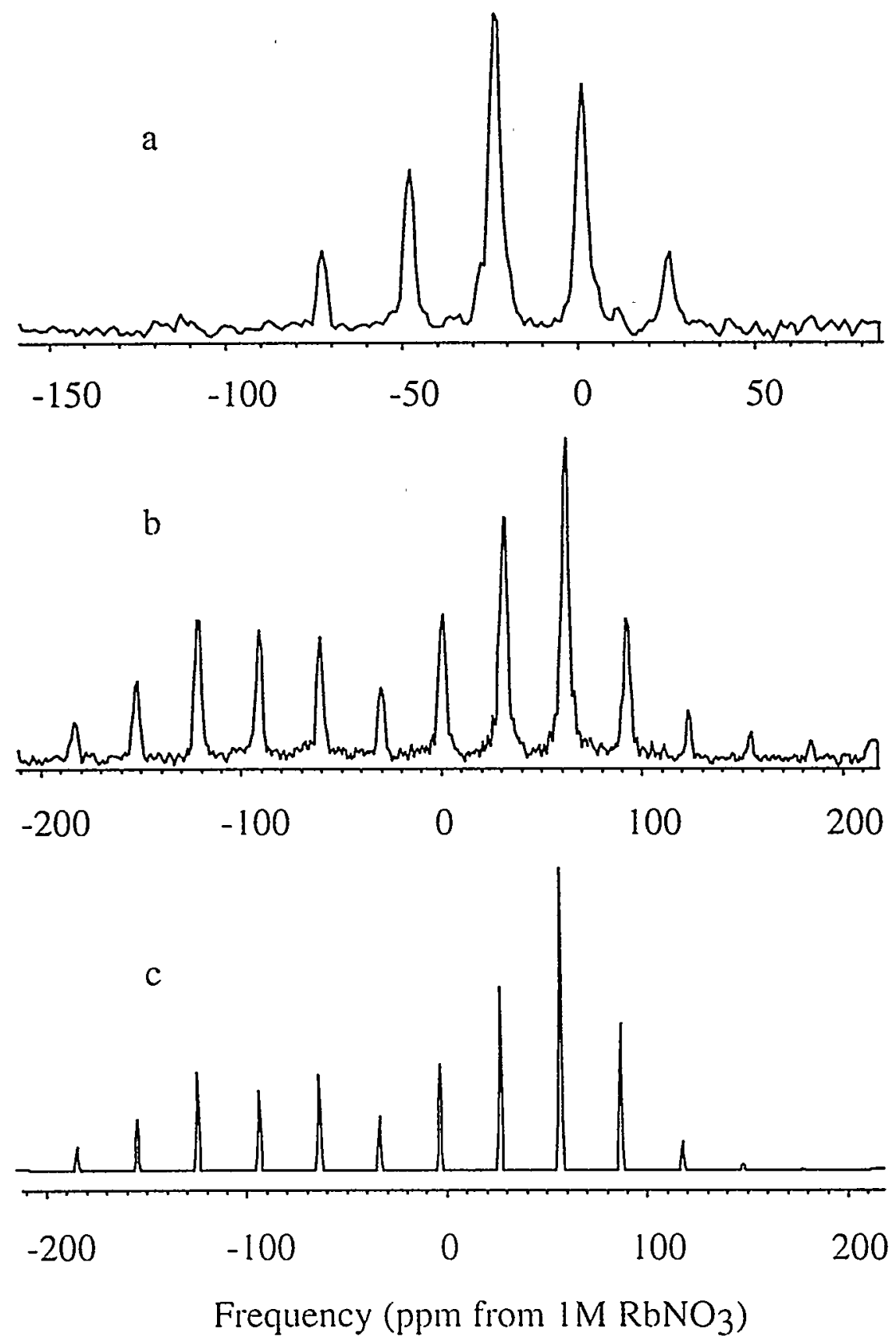

Figure 3.23: 11.TT DAS' (a), MQM SS (b) and simulated MQMAS (c) spectra for ${ }^{87} \mathrm{Rb}$ in $\mathrm{Rb}_{2} \mathrm{ClO}_{1}$. The simulation neglects the anisotropic 2nd-order quadrupolar interaction. 
since chemical shift anisotropy (CSA) still influences the spectrum.

$$
\omega_{3 / 2 \leftrightarrow-3 / 2}^{c s}=3\left(\omega_{i s o}+A_{2}^{C s}\left(\Omega^{\operatorname{css}}, \beta^{C S S}\right) P_{\cdot 1}(\cos \theta)\right)
$$

The pure (SSA dimension (triple-quantum dimension) allows one to determine chemical shift anisotropy (CSA) directly, even if it is too small to be separated from the quadrupolar interaction in a single-quantum spectrum. The method would also be more sensitive to small chemical shift anisof ropy since the CSA effect on a triplequantum spectrum is magnified by a factor of three, compared to its effect on a single-quantum spectrum. Bxperimentally, the MQVAS data can be collected using the MQMAS sequences and processed accordingly. A shearing transformation is not needed since redefinition of the crolution time is not nocessary here.

As an example, Figure 3.21 shows the $11.7 \mathrm{~T}$ RINNO 3 QVAS spectrum acquired with sample rotated at. $70.12^{\circ}$. The quadrupolar paramoters for ach of the three sites in this salt have been determined by: D.AS and MQMM:S [51, 2-1]: $\delta_{2 s o}=-27.4 \mathrm{ppm}$, $\mathrm{C}_{Q}=1.68 \mathrm{MHz}, \eta_{Q}=0.2$ for the first site. $\delta_{t, s}=-28.5 \mathrm{ppm}, \mathrm{C}_{Q}=1.94 \mathrm{MHz}, \eta_{Q}=1.0$ for the second and $\delta_{\text {so }}=-31.3 \mathrm{ppm}, \mathrm{C}_{\mathrm{Q}}=1.72 \mathrm{MH} \%, \eta_{Q}=0.5$ for the third. Using these parameters, the isotropic frepuency shifts in the triple-quantum dimension were calculated to giw -7.1 .2 . -71.4 and $-81.9 p p m$, respectively. These numbers are in the same region as the praks in the triphecfuamum spectrum. Compared with the simulated spectia in ligure 3.25, it is likely that the spectrum contains multiple overlapping well-dedined patterns. Howerer. accurate determination of the CSA parameters for this salt recplites a three-dimonsional exporiment that separates the three sites.

Experiments on ofler samples wore also performed (for example, ${ }^{23} \mathrm{Na}_{2} \mathrm{C}_{2} \mathrm{O}_{4}$, ${ }^{23} \mathrm{Na}_{2} \mathrm{SO}_{4},{ }^{8 \top} \mathrm{Rb}(\mathrm{lO})_{4}$ and $\left.{ }^{{ }^{2}} \mathrm{R} \mathrm{h}_{2} \mathrm{SO}_{4}\right)$ and in all of Ile cases. pure-absorption phase 3QVAS spectia were ohtained. The spectra all have an asymmelic triple-quantum dimension, but no clear singularilies cau be identilied to accurately determine CSA 


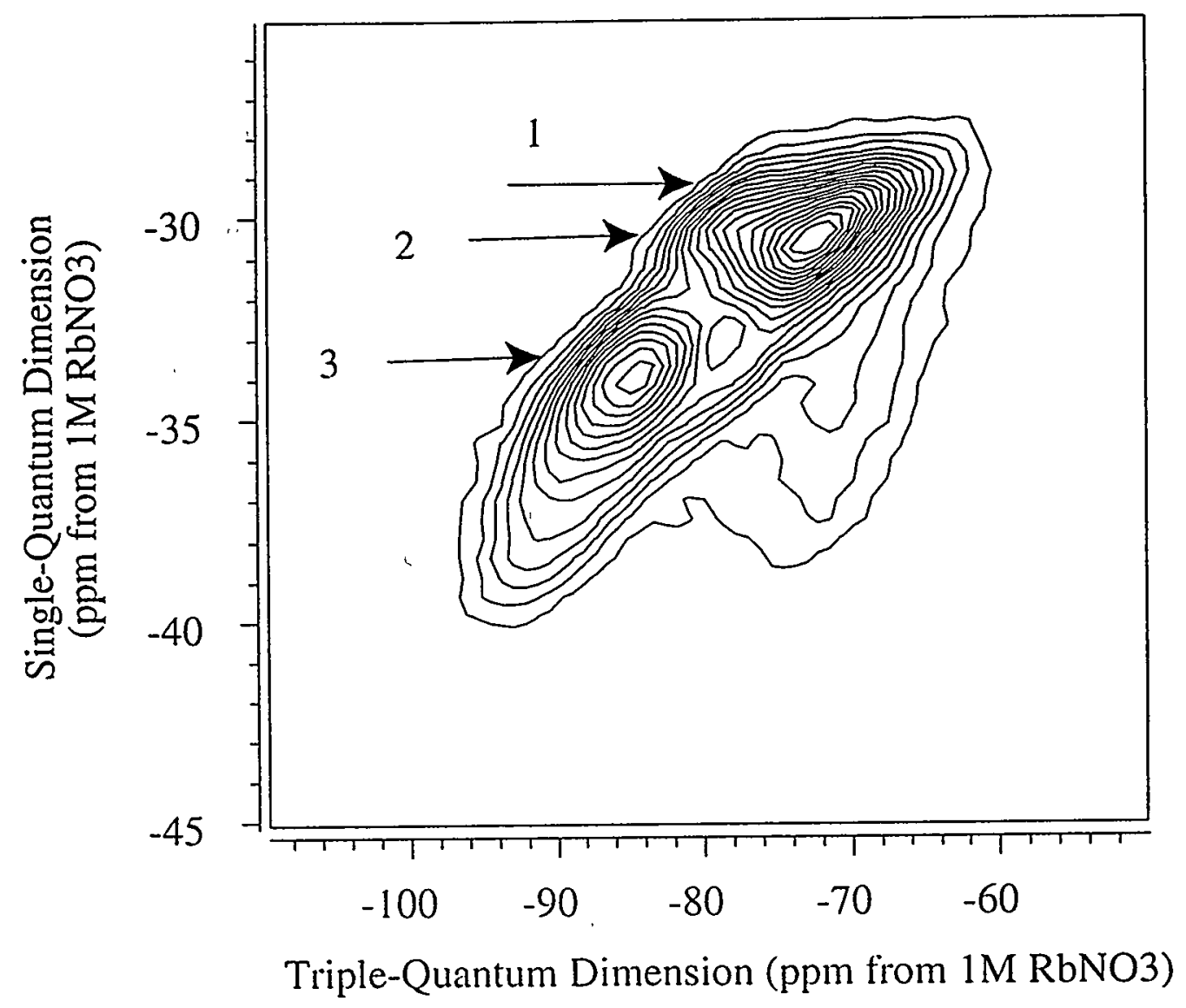

Figure 3.24: 11.tT 3QVAS spectrum of ${ }^{87} \mathrm{R.b}$ in $\mathrm{RlbNO}_{3}$.

parameters. This is most. likely due to the existence of other anisotropic interactions including dipolar coupling. For MQVAS to be useful, such interactions have to be small enough that they clo not obscure the CSA effect.

The next chapter shows that 2D switching-angle spinning (SAS) spectra for quadrupolar nuclei are more scusitive to small ratrations in Euler angles between the chemical shift and guluadrupolar principal axis sistems (PAS) [84, 86]. The tivo-dimensional 3QVAS patterus for $l=\frac{3}{2}$ nuclei may also be used to yield similar information. Figure 3.25 shows the simulated 2D 3QVAS spectra using same chemical shift and quadrupolar parameters. As one can see. the patterns show a significant dependence on the relative orientation of the l.wo lenssors, thus providing a promising method for 


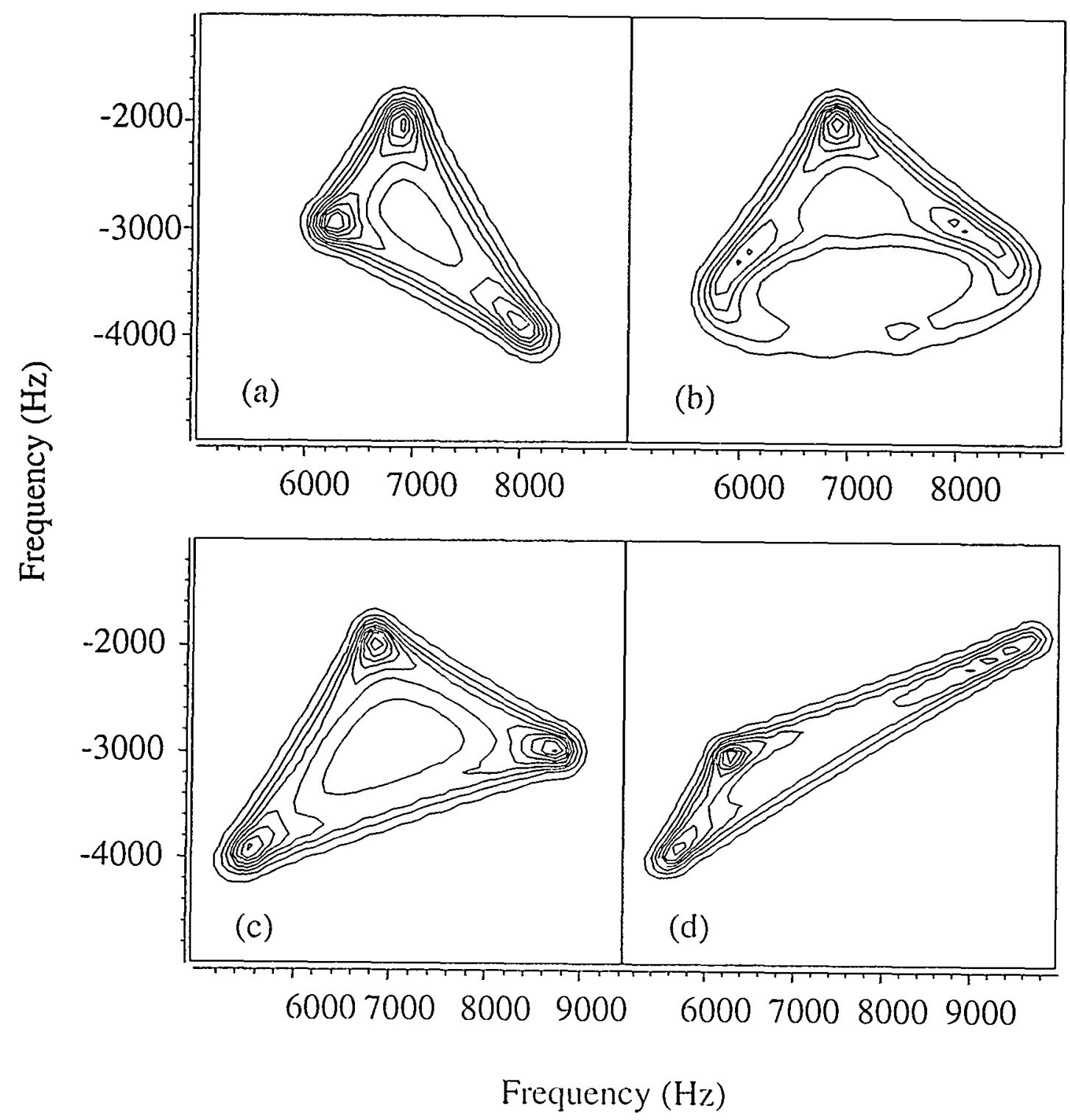

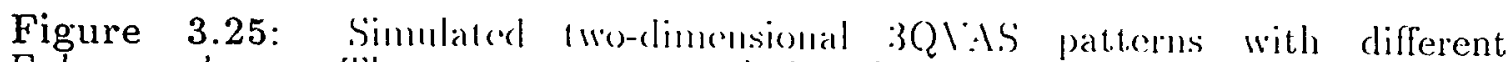
Euler angles. The parameters used in the simulations are $C_{Q}=3.0 \mathrm{MHz}$, $\eta_{Q}=1.0, \delta_{i s u}=0.0 \mathrm{ppm}, \eta_{\mathrm{cs}}=1.0,1=3 / 2, \omega_{0}=150 \mathrm{M} / \mathrm{z}$. The Euler angles are (a) $\left(0^{\circ}, 0^{\circ}, 0^{\circ}\right)(b)\left(0^{\circ}, 0^{\circ} .455^{\circ}\right)(c)\left(0^{\circ}, 0^{\circ} .90^{\circ}\right)($ d $)\left(90^{\circ} .90^{\circ} .90^{\circ}\right)$. 
quantifying the Euler angles.

\subsection{Alternatives to DAS and MQMAS}

\subsubsection{Double Rotation (DOR)}

DAS and MQMAS are two-dimensional echo like experiments that reconstruct isotropic NMR spectra for quadrupoles in the indirect dimension. Double rotation (DOR) is a simple expcriment; that does not involve this second-dimension $[87,88]$. However, it is technically more difficult since the sample is spun around two angles simultaneously. The wo angles are chosen to be the roots of the second- and fourthorder Legendre polynomials $\left(54.74^{\circ}\right.$ and $\left.30.56^{\circ}\right)$. To derive the Hamiltonian under DOR, an extra rotation transformation is involved and Eq̣n 2.84 is needed.

$$
-_{2 m}^{Q}-1_{2,-m}^{Q}=\sum_{l=0.2,1}<l 0 \mid \cdot 2 \cdot m,-m>a_{l 0}
$$

Notice that even though the other approach that expands the second-rank tensor product ( $\mathrm{Eqn} 2.76-2.82)$ works t.oo, it is very tedious to use with this extra rotation.

$a_{10}$ now has a clefinition different, from Eqn 2.85 .

$$
a_{l 0}=\sum_{n, j, k=-l}^{l} D_{n 0}^{(l)}\left(\omega_{r \cdot 1} l, O_{1}, 0\right) D_{j n}^{(l)}\left(\omega_{r \cdot 2} l, 0_{2}, 0\right) D_{l, j}^{(l)}\left(\alpha^{Q}, \beta^{Q}, \gamma^{Q}\right) \sigma_{l k}
$$

In this equation, $\omega_{r 1}$ and $\omega_{r 2}$ arc the spinning rates at two spinning angles $\theta_{1}$ and $0_{2}$. If only the time-independent terms are considered. we have $n=j=0$ and

$$
a_{10}=d_{00}^{(l)}\left(\theta_{1}\right) d_{00}^{(l)}\left(\theta_{2}\right) \sum_{k=-1}^{l} c^{-i k n^{Q}} D_{k \cdot 0}^{(l)}\left(\beta^{Q}\right) \sigma_{l k} .
$$

Notice that

$$
d_{(u)}^{(l)}=P_{l}(\cos \theta),
$$


The frequency shift under DOR can be writien as

$$
\omega^{2 Q}=\omega_{i s o}^{2 Q}+A_{2}^{Q}\left(\alpha^{Q}, \beta^{Q}\right) P_{2}\left(\cos \theta_{1}\right) P_{2}\left(\cos \theta_{2}\right)+A_{1}^{Q}\left(\alpha^{Q}, \beta^{Q}\right) P_{4}\left(\cos \theta_{1}\right) P_{4}\left(\cos \theta_{2}\right)
$$

Since

$$
\begin{aligned}
& P_{2}\left(\cos \theta_{1}\right)=0 \\
& P_{1}\left(\cos \theta_{2}\right)=0,
\end{aligned}
$$

only the first term in lign 3.93 is non\%cro (isotropic 2nd-order shift) and highresolution is achieved.

DOR has been successfully used to study a series of aluminum or oxygen containing materials $[89,90,75]$. 'The problem with DOR is that it requires sample spinning at two angles at the same time and the outer spimner can only be spun at about $1 \mathrm{kHz}$. Even with rotor synchronizalion which crates a virtual spinning specd two times as fast as the real spinning specel. the spectra are still congested with sidebands that spectral interpretation is ofton complicated.

\subsubsection{Dynamic-Angle Hopping}

Dynamic-angle hopping (1) MII) [91] is an intoresting two-dimensional experiment that rotates the sample very slowly but 10 ally removes the sidebands in the isotropic dimension. The isotropic dimension is constructed using a similar scheme as DAS and a hopping is also imsolver. The experiment is an extension of the magic-angle hopping (MAII) experimenl [!2]. The basic idea is lhat high-resolution and removal of spinning sidebands are 1 wo different goals in NAIR and can be dealt with separately. To achieve high-resolution, Ho sample does not. need to be spun very fast (DOR is an example). Fast spinning is often used since stow spinning gives extra sidebands [22, 93]. If the sidebands can be removed through other ways fast spinning is not needed. 
In DAH, high-resolution is achieved by spinning the sample first at $63.43^{\circ}$, and then at $0^{\circ}(k=5)$. The sideband is removed however, by applying five pairs of pulses at $63.43^{\circ}$. Since I have chosen not to include a complete description of spinning sideband theories in chapter 2, I will not go any cletails about how DAH gets rid of the spinning sidebands. The tiechnique does not find much application, because a DAS probe is still required and the large number of $\mathrm{RF}$ pulses used may render many samples inaccessible. 


\section{Chapter 4}

\section{Application of MQMAS to Aluminum-Containing Materials}

The use of multiple-cuantum magic-anglo spinning to study ${ }^{27} \mathrm{Al}$-containing materials is probably the most important application of this experiment so far. Aluminum, along with oxygen and silicon, is one of the most common nuclei in zeolites, minerals, glasses and other t.echuologically important, materials. Solid-state NMR is becoming increasingl! important in resolving some structural and quantification problems in these materials [9-1, 95, 96, 97, 98, 99, 78, 100, 101].

In aluminosilicatc and aluminate crystals and glasses, there are three common types of aluminum environments with dilferent aluminum coordination number. The four- and six-coodinated aluminum sites have been identified in a series of glasses $[102,103,104,105,106]$, even though the stalic and MAS spectra of ${ }^{27} \mathrm{Al}\left(\mathrm{I}=\frac{5}{2}\right)$ in glasses are often poorly resolved because of disorder and quadrupolar broadening. The four- and six-coordinalod almminmm sile appear around 60 and 0 ppm respectively, whereas the cuadrupolar compling constants for each type of sites may range from 2-3MH\% to 10.MII\%. An NMIR poak around 30ppm was also obscrved in many silicate matcrials, especially in samples prepared under high-pressure [107, 108, 109 ,

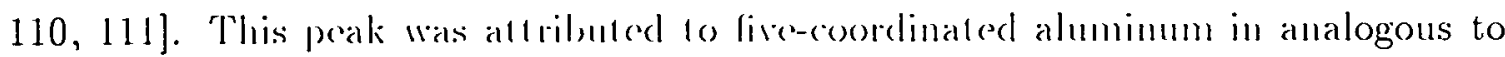
the assignment of the silicon sperea [112]. (renthongh the existence and quantification of this site remain contresersial [11:3]. The high-resolution achieved through MQMAS may shine great light on this problem. In this chapter. I will discuss some of the experiments that are performed on aluminosilicate and aluminate samples that lead to a relatively clear picture of these materials. The utility and the limitation of 
MQMAS are also discussed.

\subsection{Interpretation of MQMAS Spectra}

Before going into any detail about our ${ }^{27} \mathrm{Al}$ experiments, the interpretation of MQMAS spectra is to be discussed first. The goal of most solid-state NMR experiments is to extract useful struclural and dynamic information about the materials of interest. Such informalion is strongly coupled to some NMR parameters including quadrupolar coupling constant $\left(C_{Q}^{\prime}\right)$ and isotropic chemical shift $\left(\delta_{i s o}\right)$. For example, it was suggested that the isolropic clicmical shift of ${ }^{27} \mathrm{Al}$ or ${ }^{29} \mathrm{Si}$ directly reflects the coordination number of aluminum or silicon [114, 78]. It was also found that the quadrupolar coupling constant. $\left(\left(_{Q}\right)\right.$ [or ${ }^{17} \mathrm{O}$ in the Si-O-Si linkage is approximated by $[47,80,115]$

$$
C_{Q}(\angle S i-O-S i)=C_{Q}\left(1 S 0^{\circ}\right) \frac{2 \cos (\angle S i-O-S i)}{\cos (\angle S i-O-S i)-1}
$$

Thus the measurement of chemical shift and quadrupolar parameters may be essential in discriminating and quantifying aluminum sites with different coordination number.

The extraction of quadrupolar ( $\left({ }_{Q}\right.$ and $\left.\eta_{Q}\right)$ and chemical shift parameters $\left(\delta_{i s o}\right.$, $\delta_{C S}, \eta_{C S}$ ) may not always be obvions. Por instance, the observed DAS and MQMAS shifts are the combination of the isolropic chemical shift and second-order quadrupolar shift. Special teratment needs lo be done to sepalater the shifts from the two sources.

\subsubsection{Spectral Simulation}

Simulating the experimontal N.IIR lines is by lar the most widely used approach in solid-state NMR to get information ahout the anisotropic interactions. For quadrupolar nucleus, the simulation would idcally include up to 8 independent, parameters 
$\left(\delta_{i s o}, \delta_{C S}, \eta_{C S}, C_{Q}, \eta_{Q}, n, \phi, \gamma\right)$. 'The large mumber of parameters may overfit the data and practically, three parameters $\left(\delta_{i s o}, C_{Q}^{\prime}\right.$ and $\left.\eta_{Q}\right)$ are used in simulations. This approximation neglects ( $5 \mathrm{SA}$, but is proved adequate, especially when fast MAS is performed which minimizes the ('SA offect.

The problem with the fithing procedure is that the number of fitting parameters grows up quickly when there are multiple siles in the system. This is one of the reasons that MAS spectar of ${ }^{2 \pi} \mathrm{Al}$ are often hard to qualify and quantify. The overlap of the four-, five- and six-coordinated aluminum praks makes the simulation almost impossible in some casces. Iligh-resolution technigues such as DAS or MQMAS are then useful to differentiate distinct sites in the systom and provide initial guess of the NMR paramelers for hose sites. In the best cases (See Figures 3.9 and 3.11), when distinct, siles are resolved in l.he 1) AS or MQMAS spectrum, anch site can be simulated separately, which greally reduces the mumber of parameters in the fitting and increases the accuracy and precision of the simulation.

\subsubsection{DAS and MQMAS: Extraction of $\delta_{i s o}$ and $P_{Q}$}

It is possible to obtain $\delta_{t, \text { and }} P_{Q}$ (defined below) without resorting to the simulation method. As shown in section :3.2, hhe observed D.AS or MQQMAS frequency is the combination of the isotropic rhemical shift and the isotropic End-order quadrupolar shift.

$$
\begin{aligned}
& \delta^{D+1.5}=\delta_{\text {sst }}+\delta_{r, S i}^{2 Q} \\
& \delta^{.11 Q 2.1 / 1.5}=l_{1} \delta_{1 s_{0}}+l_{2} \delta_{1 s 0}^{2 Q}
\end{aligned}
$$

To obtain the isol ropic chemical shift and the quadrupolar conpling constant, multiplefield experiments are performed. 'The isotropic chemical shifi $\delta_{\text {sus }}$ is not dependent on the external lickd strength (in the muits of ppm): lhe second-order quadrupolar 
shift, on the other hand, is inversely proportional to the square of the $B_{0}$ field.

$$
\delta_{i s o}^{2 Q}=-\frac{3 \times 10^{6}}{40} \frac{\left(I(I+1)-\frac{3}{4}\right) C_{Q}^{2}\left(1+\frac{\eta_{Q}^{2}}{3}\right)}{\omega_{0}^{2} I^{2}(2 I-1)^{2}}=C \frac{P_{Q}^{2}}{\omega_{0}^{2}}
$$

Here,

$$
P_{Q}=C_{Q} \sqrt{1+\frac{\eta_{Q}^{2}}{3}}
$$

and

$$
C=-\frac{3 \times 10^{6}}{40} \frac{\left(I(I+1)-\frac{3}{4}\right)}{I^{2}(2 I-1)^{2}}
$$

If DAS measurements are donc al, two separate fields, one gets

$$
\begin{aligned}
& \delta_{1}^{D A s}=\delta_{i s o}+C \cdot \frac{P_{Q}^{2}}{w_{01}^{2}} \\
& \delta_{2}^{D A H}=\delta_{i s o}+C \cdot \frac{P_{Q}^{2}}{\omega_{02}^{2}} .
\end{aligned}
$$

Solving the simultancous cquations gives

$$
\begin{aligned}
& P_{Q}=\omega_{01}^{\prime} \omega_{0122} \sqrt{\frac{\delta_{1}^{D A S}-\delta_{2}^{D A S S}}{C^{\prime}\left(\omega_{02}^{2}-\omega_{01}^{2}\right)}} \\
& \delta_{i s o}=\frac{\omega_{01}^{2} \delta_{1}^{D A S}-\omega_{02}^{2} \delta_{2}^{D A S}}{\omega_{01}^{2}-\omega_{02}^{2}} .
\end{aligned}
$$

One should notice that to obtain $P_{Q}$ and $\delta_{\text {iso }}$ all we need is two linearly independent equations of $l_{Q}$ and $\delta_{i s,}$. Therefore, onc of the equalions may come from DAS (or MAS), and the other one from MQMAS. To see this. recall that

$$
\begin{aligned}
& \delta^{D A H}=\delta_{i s u}+C \frac{P_{Q}^{2}}{\omega_{0}^{2}} \\
& \delta^{M Q Q M A H}=k_{i} \delta_{i s,}+\left(k_{i 2} \frac{P_{Q}^{2}}{w_{0}^{2}} .\right.
\end{aligned}
$$




\begin{tabular}{|c|c|c|c|c|c|c|}
\hline Site & $\delta_{M A S}^{9.4 T}(\mathrm{ppm})$ & $\delta_{3 Q M A S}^{9.7 T}$ & $\delta_{\Lambda I A S}^{11.2 T}$ & $\delta_{3 Q A \Lambda A S}^{11-T T}$ & $\delta_{i s o}$ & $P_{Q}(\mathrm{MHz})$ \\
\hline T1 & $58.6 \pm 2.0$ & $-34.2 \pm 0.2$ & $59.6 \pm 1.5$ & $-34.0 \pm 0.2$ & $61.0 \pm 0.7$ & $2.07 \pm 0.50$ \\
\hline $\mathrm{T} 2$ & $59.7 \pm 2.0$ & $-36.4 \pm 0.2$ & $61.8 \pm 1.5$ & $-35.7 \pm 0.2$ & $63.9 \pm 0.6$ & $2.58 \pm 0.50$ \\
\hline $\mathrm{T} 3$ & $66.1 \pm 2.0$ & $-39.1 \pm 0.2$ & $67.2 \pm 1.5$ & $-38.4 \pm 0.2$ & $69.2 \pm 0.7$ & $2.34 \pm 0.50$ \\
\hline
\end{tabular}

Table 4.1: Isotropic shift.s and c|uadrupolar coupling parametcrs for leucite from 11.7 T and 9.4 T 3QMAS experiments, cerived from 3QMAS and MAS peak positions.

If the observed shifts in the single- and triple-cutantum spectra are available, one would get

$$
\begin{aligned}
& P_{Q}=\omega_{i 10} \sqrt{\frac{l_{1} \delta D A S}{C^{\prime}\left(k_{1}-j_{12}\right)}}
\end{aligned}
$$

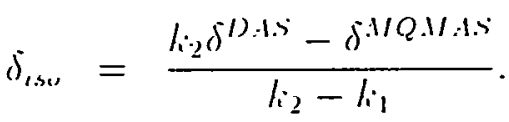

Figure 4.1 shows how lign 4.13 and Feqn 4.14 can be used to extract both $P_{Q}$ and $\delta_{i s o}$ from a single MQMASS experiment. In the two-dimensional ${ }^{27} \mathrm{Al}$ spectrum of leucite at 11.7'T, hare isotropic praks are obserred in both climensions. Inserting the observed shifts for ach site in both dimension into Eqn 1.13 and Eqn 4.14, the isotropic chemical shift and guadrupolar product. for each sitc can be generated.

To improve the overall accuracy and precision for the measurement of $P_{Q}$ and $\delta_{i s o}$, multiple experiments should lor performed al many fiedels. This opens the possibility

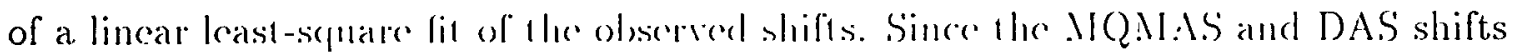
are scaled differently preprocessing of the observed shifts is ueeded before the fitting can be done. 'lo do this. He obserered shift is first written in the more general form as follows.

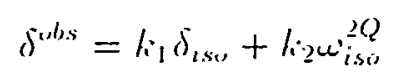

For DAS', $k_{1}=k_{2}=1$. Dividing boll sides of the cequation by $k_{1}$, we get an equation 


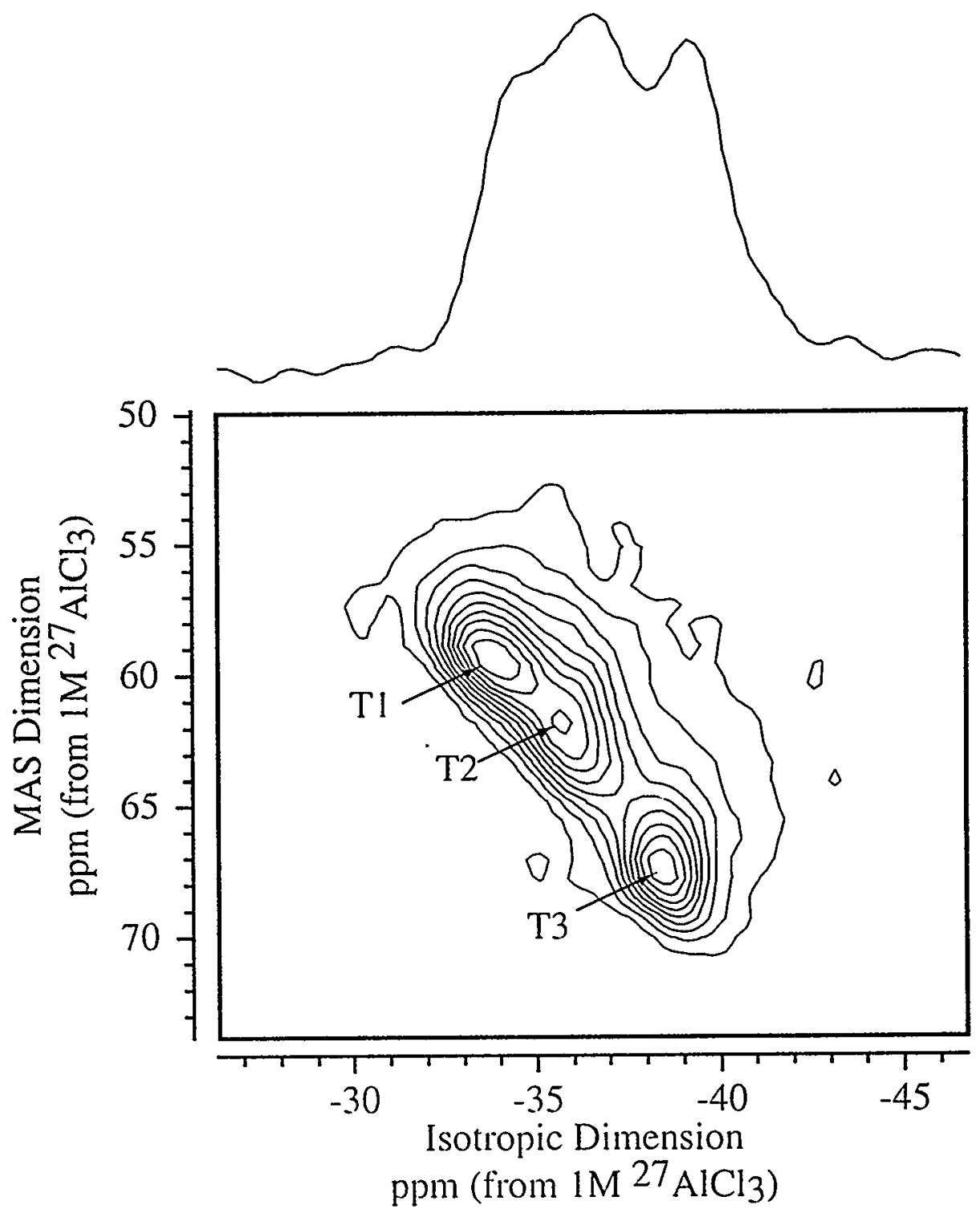

Figure 4.1: Conlour plot of ${ }^{22} A 1$ 3Q.MAS NiMR spectrum for leucite. The 1D MQMAS spectirum on top is the projection onto the isotropic dimension. 
that can be used for the lincar regression:

$$
\frac{\delta^{i b s}}{k_{1}}=\delta_{i s o}+\frac{k_{2}}{k_{1}} \omega_{t, S o}^{2 Q}=\delta_{i s i}+\frac{C k_{2}}{k_{1} \omega_{0}^{2}} P_{Q}^{2} .
$$

Plotting $\frac{\delta^{o b s}}{k_{1}}$ versus $\frac{C k_{2}}{k_{1} \omega_{0}^{2}}$ would give a straight. line, whose slope is the square of $P_{Q}$, and the interception is the isotropic chemical shift. Figure 4.2 demonstrates this strategy for leucite, where the $9.4 \mathrm{~T}$ and $11.7 \mathrm{~T}$ data are combined and used in the fitting. This linear regression gives significant improvement on the overall errors, and the fitting results are reported in lable 4.1 .

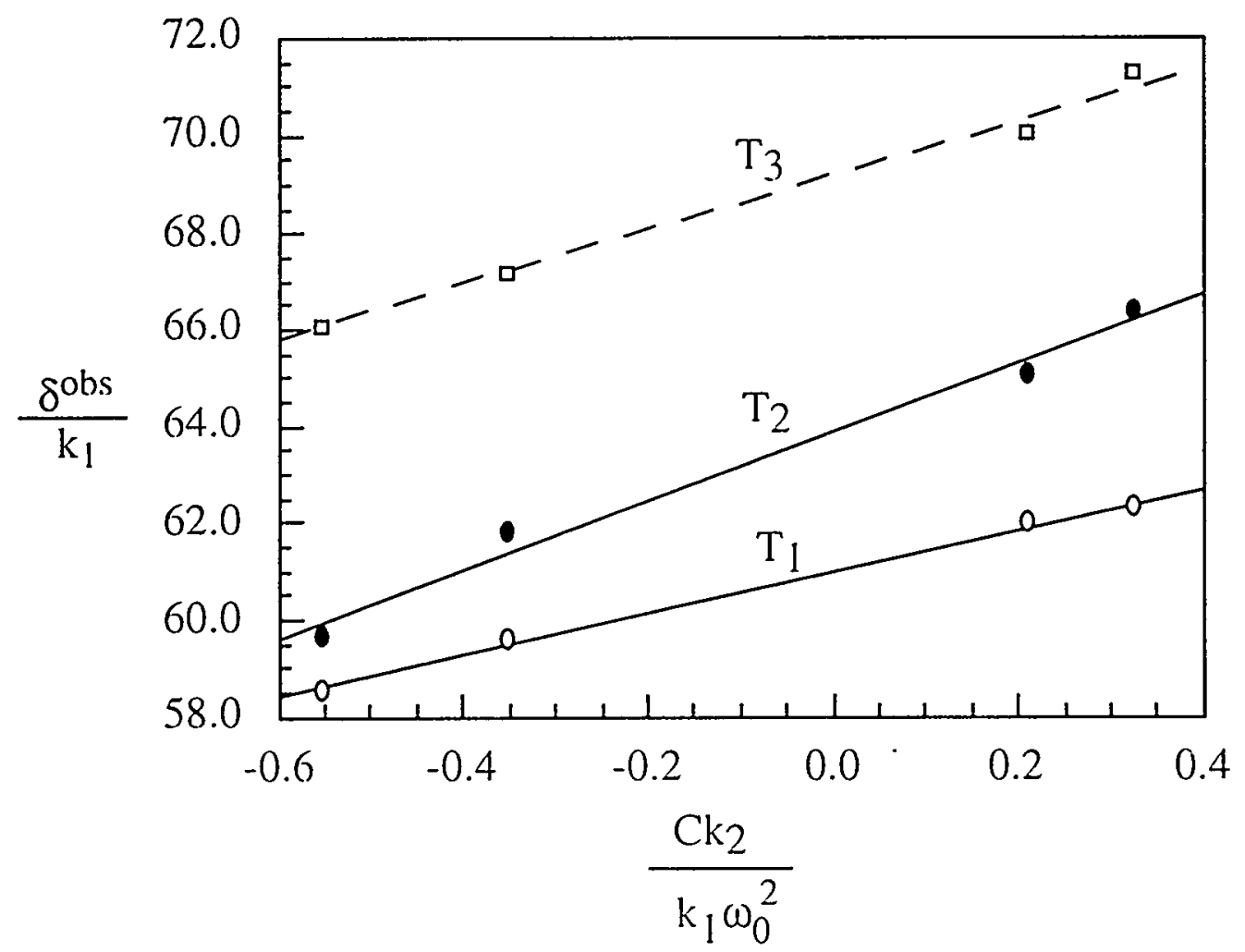

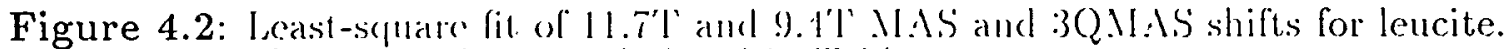
The data and fitling lesults are labulated in lable 1.1.

It is important. to note that it is impossible to extract ('Q and $\eta_{Q}$ from only the isotropic shifts in 1):LS or MOQNAS spectra. Such information comes from the simulation of the S.MIR linestape. In this respere. MQQMAS has a subtle advantage 
over DAS. The anisotropic spcclrum in DAS (assuming $k=1$ ) is a VAS spectrum with sample rotating art $79.19^{\circ}$ or $37.38^{\circ}$. The anisotiropic spectrum in MQMAS, on the other hand, is a MAS spectrum. Simulating the MAS spectrum gives more accurate isotropic chemical shift and quadrupolar parameters for two reasons. First, the chemical shift anisotropy does not distort the MAS lineshape. Second, at one of the two $k=1$ DAS angles, the NMR spectrum has a long tail (Figure 3.4), whose intensity is so low that can not be precisely measured in experiments and reproduced by simulation. This long lail, however, determines the magnitude of the quadrupolar coupling constant and introduces significant. uncertainties. The MAS spectrum does not have this problem and can often be accurately simulated.

\subsubsection{Quantification}

Sometimes, not only the NMIR lineshapes for each site, but also the quantification for the sites are important. Since MIQMAS is not quantitalive, the isotropic spectra can seldom be used for quanlitative purpose unless all the sites have very similar quadrupolar coupling constants. There has been some work showing that DAS is relatively cquantitative, if the $T_{1}$, for different sites are similar [116].

A better approach for quantification may be a combination of the high-resolution techniques with the simulation of stalic or MISS spectra. [4t]. In this approach, DAS or MQMAS provide initial estimale of the number of sites in the sample, and the chemical shift and quadrupolar parameders for cach site. These parameters are then fed to a fitting program to fit the static or MAS spectra. In the case that each site in the sample has well-clefined lineshape this approach is superior to other approaches that use the information from onle one Irchnique. 


\subsection{Experiments}

\subsubsection{Sample Preparation}

The sample of the natural framework silicate mineral leucite ( $\mathrm{KAlSi} \mathrm{O}_{6}$, from the Roman volcanic province) has been previously studied in detail by ${ }^{29} \mathrm{Si}$ MAS NMR [117]. Several samples of crystalline anorthite $\left(\mathrm{CaAl}_{2} \mathrm{Si}_{2} \mathrm{O}_{8}\right.$, another framework silicate) were prepared by the method described in a detailed study of Si/Al disorder [118]. A glass of this composilion was prepared by molting of the oxides at $1650^{\circ} \mathrm{C}$ for about I hour followed by air curenclung. Several portions of the glass were then crystallized by rehouling al $1400^{\circ}$ (' for cilher 1 minules (sample 1 ) or 65 hours (sample 2). Powder X-ray diflraction on lliese samples. and ${ }^{29} \mathrm{Si}$ MASS NMR spectra, showed only anorthite to be prescut. The latter spectra closely resemble those of Phillips et al. [118] for samples crystalli\%erd for 15 mimutes and 179 hours, respectively, and thus have a smaller difference in the extent of disorder than expected (presumably because of vagaries of thermal history and unclation kinctics). A sample of natural kyanite $\left(\mathrm{Al}_{2} \mathrm{SiO}_{5}\right.$, locality $\mathrm{m}$ known) 1 as also selected in order to test the relative excitation efficiencies for $\Lambda$ siles with widely raring quadrupolar coupling constants. A glass of

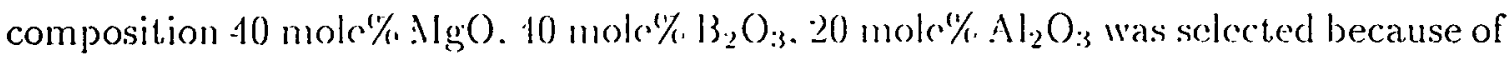
its large concentrations of four-. five- and six-coordinated alumimum as determined previously by ${ }^{27}$ Al MlS XIIR, and was also propared by mixing and melting the oxides.

\subsubsection{NMR Spectroscopy}

The MAS experiments al 9. I'T were performed on a modified Varian VXR-400S spectromeler with a 5mm high-spered MAS probe from Doty Scientific, Inc., with spinning rales of aboul. II kll\%. $\$ 111.7 \mathrm{~T}$, experiments were performed on a Chemagnetics spectromerer using the same probe or a home-luth D.1S probe described 
in the preceding chapter. Spin-latice relaxation times $\left(T_{1}\right)$ were measured with the saturation-recovery mel.hod, and delay times in 3QMAS experiments were chosen to be at least $3 T_{1}$ to assure nearly complete relaxation. The low efficiency of the triplequantum excitation, and the two-dimensional datia acquisition, resulted in typical total acquisition times for the spectra shown here of 12-24 hours, much longer than times typically required for ID, single-cuantum MAS experiments (typically a few minutes for ${ }^{27} \mathrm{Al}$ ). Useful 3QMAS spectra can generally be obtained in somewhat shorter times of a few hours.

The pulse sequence used was the shifi-echo 3QMAS sequence and is shown in Figure 3.10. The first and sccond pulses are solid-state $540^{\circ}$ pulses applied with the highest allowable powor $(50-60 \mathrm{k} / \mathrm{l} \%)$. The third pulse is a $180^{\circ}$ pulse applied with lower power level and is approximalcely $1.5-20 / 4$ in duration. The $l_{1}$ period was selected to have a dwell time which lias equal to the desired /, dwell time (after complete processing) multipliod by $\frac{19}{31}$. 'This factor arises from the scaling of observed shifts (Table 3.1). The MAS $l_{2}$ spect.ral wich was usually $6-20 \mathrm{kHz}$ while in the $t_{1}$ dimension it was usually 6-15 kll\%. Isually 40-100 $\iota_{1}$ points were required to obtain spectra without truncation artilacl.s. 'The delay between the second and third pulses was set to values ranging from 1-3) ms (10-30 rolor cycles). In the roferencing stage, the offset in the isotropic climension (the ppm value of the center of the resulting $t_{1}$ dimension spectrum ) need to be multiplied by $\frac{l i-3}{k+1}$ or $-\frac{17}{31}$.

The determination of the isol ropic chenical shift $\left(\delta_{i s o}\right)$ and quadrupolar coupling product $P_{Q}$ was done using the approach clescribed at the beginning of this chapter. When possible, MAS prak shapes in slices of the 2I) spectla were fitted with a

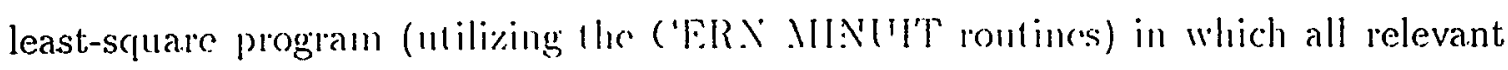
parameters in the $M I A S$ prak shape $\left(C_{Q}, \|_{Q}, \delta_{i s o}\right.$, integrated intensity, Lorentzian and Gaussian broadening) were allowed to wary. In general, the two methods produced 
similar results, althongh the latice approach may allow $\eta_{Q}$ and $C_{Q}$ to be derived in addition to $P_{Q}$.

\subsection{Results}

\subsubsection{Leucite}

The leucite MQMAS spoedrum has becn shown in Figure 4.1 to demonstrate different strategies in giving useful NMIR parameters. This mineral has a complex structure with there cristallographically distinct tel.rahedral sites ( $\left.{ }^{\top}{ }_{1},{ }^{r} T_{2}, T_{3}\right)$. Because of the complexily of the ${ }^{29} \mathrm{Si}$ spectra (as many as 15 overlapping peaks), and

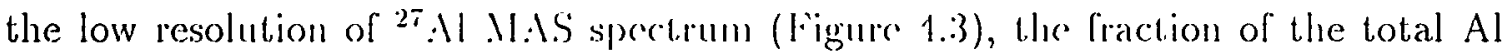
on each site remains impreciscly known. Models of essentially identical ${ }^{29}$ Si spectra

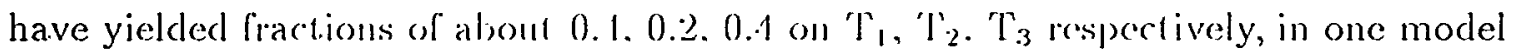
[117], 0.25, 0.50, 0.25 in a second model [119] and 0.50. 0.25. (0.25 in a third [120].

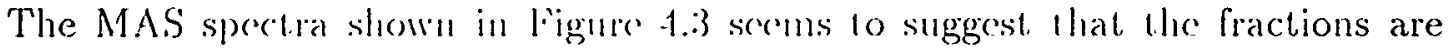
$0.5,0.25,0.25$, consistoul with andicr allalysis of ${ }^{27}$ :Il MAS data by others [121]. However, this conchusion is based on the assumption that the guadrupolar coupling

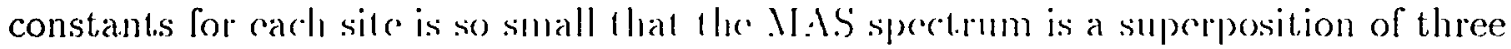
Gaussian peaks. This ma! not be true since intensily in one peak may come from the shoulders in the quadrupolar lineshape of ofher praks. The ${ }^{2 \pi} \mathrm{N}$ 2D 3QMAS spectrum of lencito (Figure 1.1) shows 3 parlially orerlapping peaks corresponding to the there siles. 'l'he projection in llar isolropic dimension shows considerably better resolution than MAS spectra in Figure 1.3. ('ompared to the MAS spectra, the 3QMAS data are more definitive in moling ont any influcner of second-order quadrupolar coupling on pakk shape. Fitting lhe projection with three Gaussian

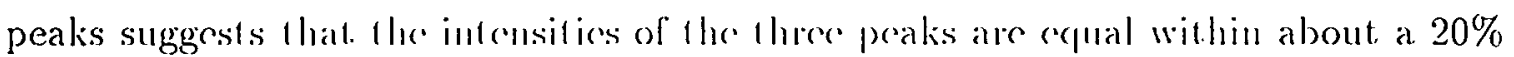
error. Residual broadening. presumaluly due lo hle disoredered arrangement of second- 


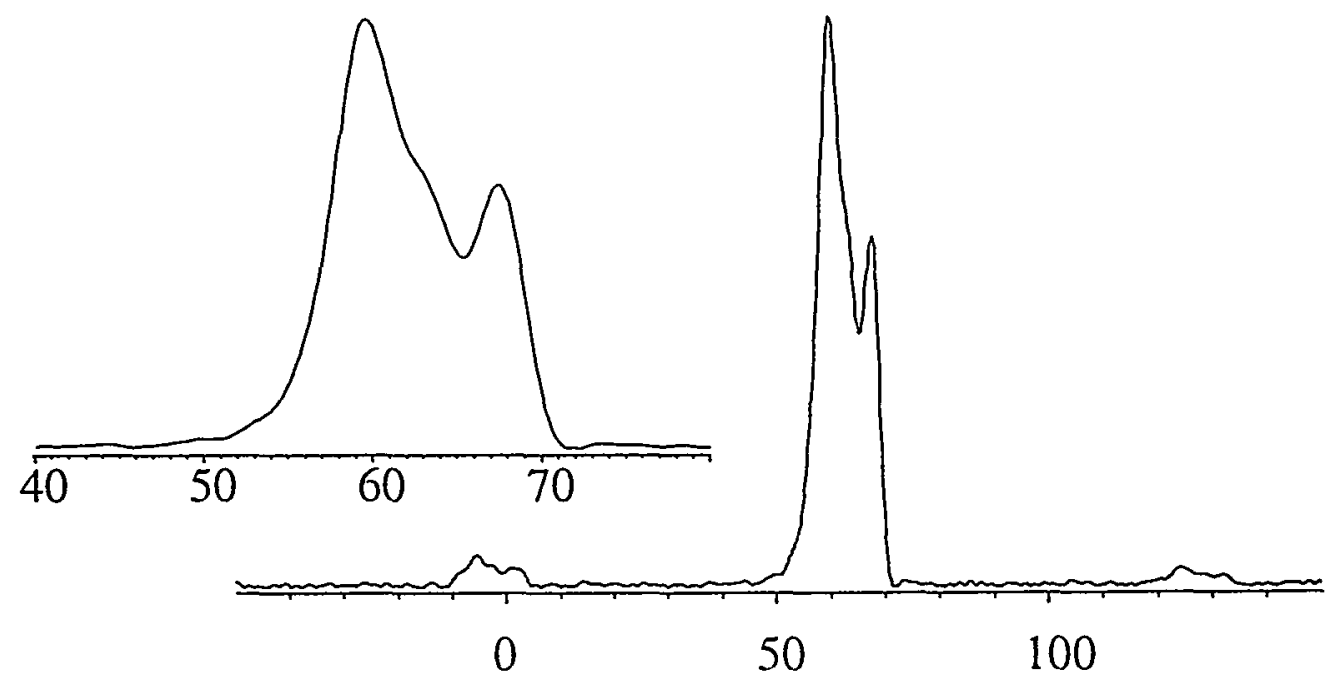

Frequency (ppm from $1 \mathrm{M} \mathrm{RbNO}_{3}$ )

Figure 4.3: ${ }^{2 \pi} \mathrm{Al}$ MAS spectia of Lencile. The spectrum was taken with the Hahn-echo sequence. The spectral region $40-80 \mathrm{ppm}$ is expanded to show the three partially resolved peaks.

neighbor cations and a resulting distribution of isotropic chemical and quadrupolar shifts, appears to limit the ultimate resolution.

Imperfect, site excitation has the potential to be quite significant in $3 Q M A S$ experiments, making quantification of intcusities complex. Even though a long pulse is capable of transferring coherence from a zero- to a triple-quantum state, the effciency of this process is highly dependent on $C_{Q}$ and on the overall RF field strength. An assumplion of uniform excitalion is thus most. likely to be valid if $C_{Q}$ values for different siles are similar. Fxact $C_{Q}$ valuess are not known for leucite, but data for isotropic chemical shifts and for l'Q have be cxtracted and shown in Table 4.1 from the two-dimensional N.MR spectra al !).1' and II.tT'. The chemical shifts agree well with values previously derived from MAS spectra including satellite siclebands, and $P_{Q}$ data are consistent. with previous rongh estimates of $1-2 \mathrm{MHz}$ [121]. The close similarity of the $P_{Q}$ values for the three peaks suggests that in this case intensities in the 3QMAS' experiment are likely 10 bre (uantitative and thus imply site occupancies 
that are somewhat discrepant from previous models. (iiven the disagreements among existing models, however, the significance of their diffrences with the present data are uncertain.

\subsubsection{Kyanite}

Kyanite was studied to further assess the cuantification of 3QMAS peak intensities. The mineral contains 4 copally populated octahodral Al sites, with $C_{Q}$ values of $10.0,9.4,6.5$, and $3.7 \mathrm{MH} \%$. The MAS spectrum is contrasted with the isotropic projection of the 3QMMSS spertrum in Figure 4.4. The resolution in the latter is dramatically increased: scparation of the poaks in the latter is enhanced by the large range in $C_{Q}$, and peaks are much narrower because of the full averaging of the secondorder quadrupolar broadening. Even siles with very large $C_{Q}$ values are excited and observed. However, it, is clear that observed intensilies are systematically reduced with increasing $C_{Q}$, suggesting that callion is required in materials where ranges of $C_{Q}$ are large or are unknown.

\subsubsection{Crystalline Anorthite}

Anorthite is an excellont test for ${ }^{2 \pi} \mathrm{Al}$ spectral tesolution: it has eight crystal-

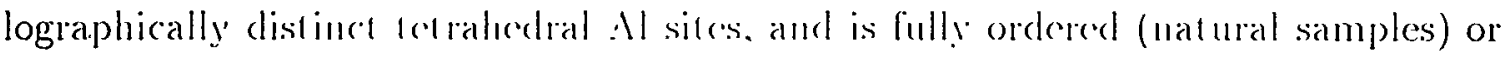

nearly so (symhloric samples). ('Q and ye values for all siles have been reported from single crystal data. but isol ropie chemical shifts are not known because ${ }^{27} \mathrm{Al}$ MAS specta are compledely muresolred. 3Q.M. IS dala at 11.7 T for more ordered crystalline anorthite are shown in ligure l.j. 'The speectrum is complex, but contains a number of significant, resolvable foatures. 'The !).l'l' spectrum is essentially the same in overall appearance with slight shifts. Results for the somewhat less ordered crystals are very similar, if porlapes slighlly less wroll-resolved. and have not been 

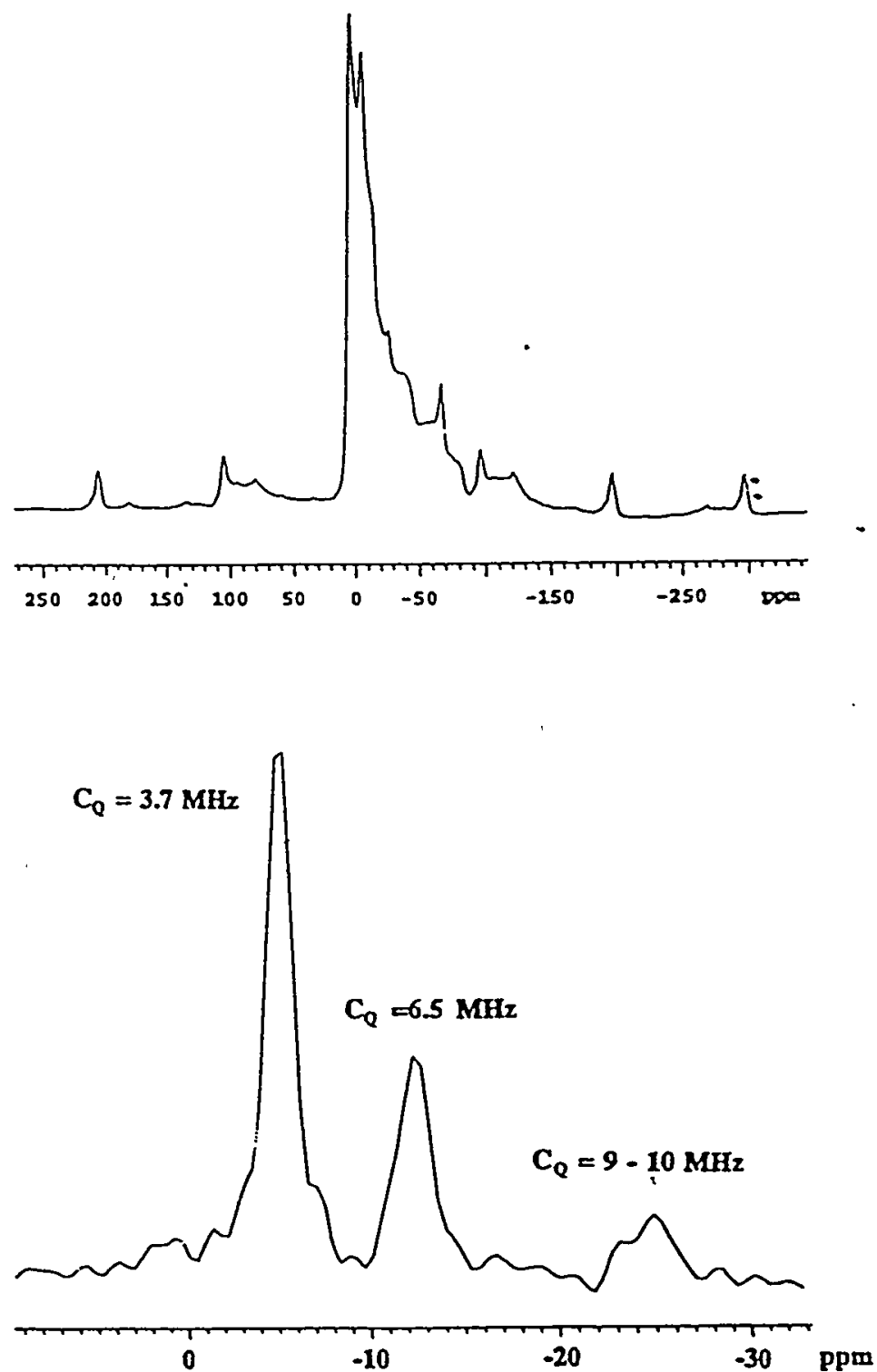

Figure 4.4: (above) ${ }^{27} \mathrm{AI} M \mathrm{AS}$ spectrum for kyanite; (below) Isotropic projection of 3QMAS spectrum. Labcls indicate $P_{Q}$ for each site. 
analyzed in cletail. Both approaches described at the beginning of this chapter are taken to analyze the data. In both, slices through the 2D spectra at the positions of obvious spectral features were laken (Figure 4.6). In the first approach, the peak

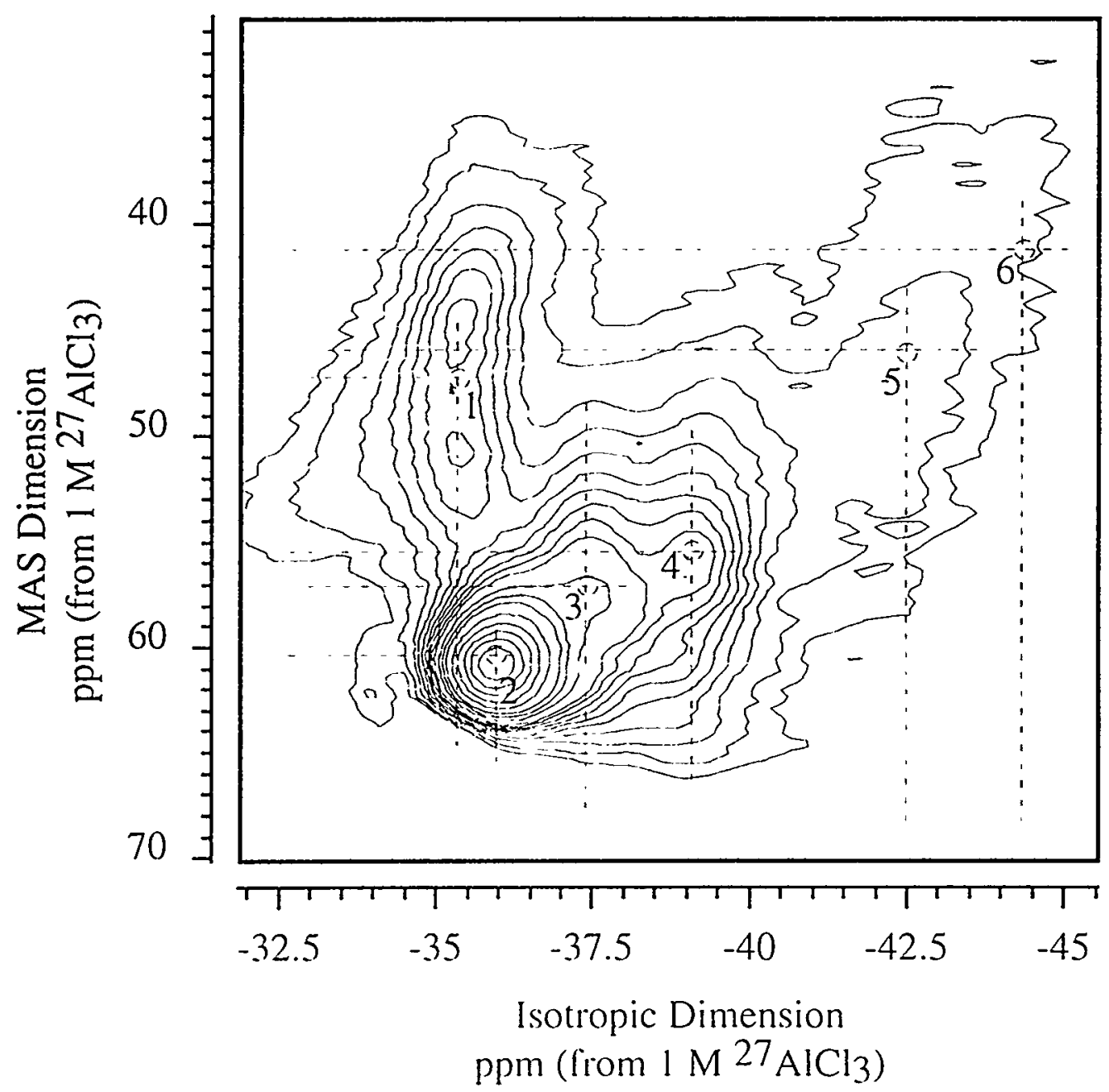

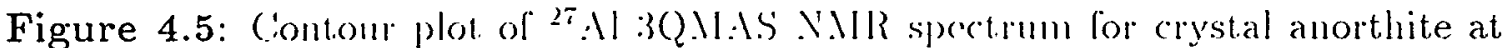
11.7T. The number points show the position of singularitios. Hhrough which slices were taken for simulations.

position in the $\omega_{1}$ dimension and the conter of gravity in the w2 (MAS) dimension were determined, and $\delta_{t, s,}$ and $P_{Q}$ were calculated using Equ 4.13 and 4.14. Results for the data at 9.4T and $11.7 \mathrm{~T}^{\mathrm{T}}$ are show'n in Tables 4.2 and 4.3 , and are consistent with each other within estimated uncertainties. 


\begin{tabular}{ccccc}
\hline Peali & $\delta_{M A S}(\mathrm{pPm})$ & $\delta_{3 Q M A S}(\mathrm{ppm})$ & $\delta_{\text {iso }}(\mathrm{ppm})$ & $P_{Q}(\mathrm{MHz})$ \\
\hline 1 & $48.0 \pm 3.0$ & $-35.4 \pm 0.2$ & $58.4 \pm 1.1$ & $5.43 \pm 0.50$ \\
2 & $61.0 \pm 1.0$ & $-36.0 \pm 0.2$ & $63.9 \pm 0.4$ & $2.88 \pm 0.33$ \\
3 & $58.0 \pm 3.0$ & $-37.4 \pm 0.2$ & $64.4 \pm 1.1$ & $4.26 \pm 0.63$ \\
4 & $55.0 \pm 3.0$ & $-39.1 \pm 0.2$ & $65.2 \pm 1.1$ & $5.38 \pm 0.50$ \\
5 & $47.0 \pm 3.0$ & $-42.5 \pm 0.2$ & $66.2 \pm 1.1$ & $7.37 \pm 0.37$ \\
6 & $40.0 \pm 5.0$ & $-44.4 \pm 0.2$ & $65.8 \pm 1.9$ & $8.54 \pm 0.52$ \\
\hline
\end{tabular}

Table 4.2: Isotropic shifts and quadrupolar coupling parameters for crystalline anorthite from 11.7T $3 Q M A S$ experiments, derived from 3QMAS and MAS peak positions.

\begin{tabular}{ccccc}
\hline Peak & $\delta_{M / A S}(\mathrm{ppm})$ & $\delta_{3 Q M A S}(\mathrm{ppm})$ & $\delta_{\text {iso }}(\mathrm{ppm})$ & $P_{Q}(\mathrm{MHz})$ \\
\hline 1 & $40.8 \pm 2.0$ & $-37.1 \pm 0.3$ & $57.1 \pm 0.8$ & $5.53 \pm 0.21$ \\
2 & $59.0 \pm 1.0$ & $-36.2 \pm 0.2$ & $63.4 \pm 0.4$ & $2.83 \pm 0.20$ \\
3 & $56.0 \pm 2.0$ & $-37.1 \pm 0.3$ & $63.3 \pm 0.8$ & $3.64 \pm 0.32$ \\
4 & $51.1 \pm 3.0$ & $-39.1 \pm 0.3$ & $63.8 \pm 1.1$ & $4.80 \pm 0.36$ \\
5 & $36 .(i \pm 1.0$ & $-4.5 .6 \pm 0.3$ & $65.9 \pm 1.5$ & $7.28 \pm 0.32$ \\
6 & $25.0 \pm 5.0$ & $-49.0 \pm 0.3$ & $65.5 \pm 1.9$ & $8.56 \pm 0.33$ \\
\hline
\end{tabular}

Table 4.3: Isotropic shifts and quadrupolar coupling parameters for crystalline anorthite from 9.4T 3QM $\triangle S$ exporiment.s, derived from 3QMAS and MAS peak positions.

MAS peak shapes in slices of the 2D spectra; were also simulatied as described in the experimental section. For example, the slice projected from -34.5 to $-36.5 \mathrm{ppm}$ in the $\omega_{1}$ dimension (which contains two distinct sites) is shown in Figure 4.6. The simulated spectrum agress woll. with all singularitics appearing in the $\omega_{2}$ dimension of the experimental data as expected. (One possible limitation of this approach is distortion of the $\omega_{2}$ dimension (MAS) peak shape duc to non-uniform excitation of nuclei in crystalliles wilh different oriculation, but we do nol expect, this problem to be severe in lhis case. Rosults of simulations are shown in Table 4.4. The fit allows assignment of at. least. five fortures in the spectra to particular crystallographic sites, based on published single crystal data ('Table 4.1). 


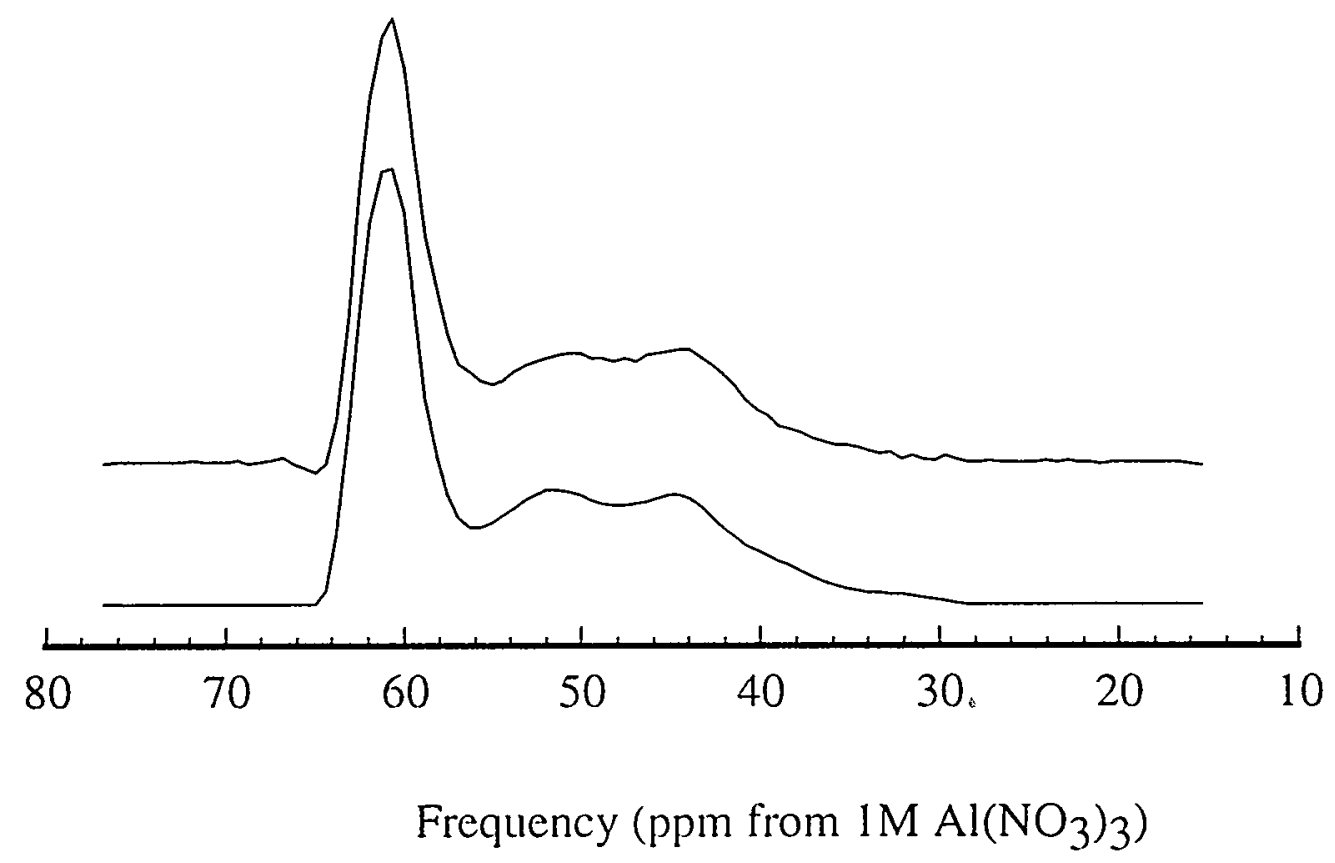

Figure 4.6: MAS Projection from -31.5 10 -36.5 ppm in the isotropic dimension of the $11.7 \mathrm{~T}$ 3QMAS spectrum of anorthite. The simulation of this slice was fit and the parameters are those for praks 1 and 2 as shown in Table 4.4 .

A sixth fealure, at. the low frequency side in $w_{1}$, can be simulated with parameters that are closest to llose experted for the 0ri0 site, but could probably also be attributed to $0 \% 00\left(C_{Q}=7.4 \mathrm{MII} \%\right)$ or $10 \mathrm{m000}\left(\mathrm{C}_{Q}=0.3 \mathrm{MIIz}\right)$. In fact, the whole tail of the spectrum in this region could well bre comprised of poorly resolved signal from the three poaks will largest ('Q . Is moled above for kyanite, peaks for sites with relatively large $C_{Q}$ are expected lo hare reduced intensilies as well as greater width in the w2 dimension, and thus are cxpected to be relatively difficult to observe with 3QMAS. 'The broad foature on he high frepuency (w) side of the tallest peak is also likely to be due 10 an unresolved pak, again possibly one of the unassigned peaks with large $C_{Q}$. In general. the agreement between the result.s for $\delta_{\text {sso }}$ and $P_{Q}$ of the two approaches 10 assigning spectral foatures is rexcellent.

The estimated isotropic chrmical shift.s for the five relatively well-constrained sites are plotted in Figure $1 . \bar{t}$ as a function of the mean intortetrahedral (Si-O- 


\begin{tabular}{|c|c|c|c|c|c|c|}
\hline \multicolumn{7}{|c|}{ Powder Resultis } \\
\hline Peak & $\delta_{3 Q M A S}^{o b s}(\mathrm{ppm}$ & $\int_{3 Q M A A S}^{p r e d}(\mathrm{ppm})$ & $\delta_{i s o}(\mathrm{ppm})$ & $C_{Q}(\mathrm{MHz})$ & $\eta_{Q}$ & $P_{Q}(\mathrm{MHz})$ \\
\hline 1 & -35.3 & -37.2 & 60.6 & 5.76 & 0.45 & 5.95 \\
\hline 2 & -35.9 & -3.5 .8 & 63.6 & 2.66 & 0.53 & 2.78 \\
\hline 3 & -37.3 & -37.9 & 64.7 & 4.39 & 0.51 & 4.58 \\
\hline 4 & -39.1 & -39.0 & 65.6 & 4.87 & 0.62 & 5.17 \\
\hline 5 & -42.2 & -40.3 & 66.3 & 6.58 & 0.70 & 7.10 \\
\hline 6 & -44.0 & -45.0 & 66.2 & 8.19 & 0.65 & 8.75 \\
\hline \multicolumn{7}{|c|}{ Single Cirystal Results and Assignments } \\
\hline \multirow{2}{*}{\multicolumn{7}{|c|}{\begin{tabular}{ccc}
\multicolumn{3}{c}{ Single Ciystal } \\
$C_{Q}(\mathrm{MHz})$ & $\eta_{Q}$ & $P_{Q}(\mathrm{MHz})$
\end{tabular} site }} \\
\hline & & & & & & \\
\hline 1 & 5.5 & 0.42 & 5.7 & $m 0 i 0$ & $145.4^{\circ}$ & \\
\hline 2 & 2.6 & 0.66 & 2.8 & $m \approx i 0$ & $137.9^{\circ}$ & \\
\hline 3 & 4.4 & 0.5 .3 & 1.6 & 0000 & $138.0^{\circ}$ & \\
\hline 4 & 4.9 & 0.75 & 5.83 & $m: \approx 00$ & $1.33 .5^{\circ}$ & \\
\hline 5 & 6.8 & 0.65 & 7.3 & $00 i 0$ & $1.31 .1^{\circ}$ & \\
\hline 6 & 8.5 & 0.66 & 9.1 & $0 \approx i 0$ & $132.0^{\circ}$ & \\
\hline
\end{tabular}

Table 4.4: Results from filling l.lic MAS' projections (slices) out of the 3QMAS NMR spectrum a.t 11.7' Tor crystalline anorthitic, compared with previous results from single crystal NMR [122] and with moan Si-O-Al bond angles from the x-ray diffraction structure. Uncertaintics in filted $C_{Q}$ values are about $0.5 \mathrm{MH} z$; in $\eta_{Q}$ about 0.2 , and in $\delta_{i s o}$ about 1 to $2 \mathrm{ppm}$.

Al) angle. As expected from previous MAS NMR studies of both ${ }^{29} \mathrm{Si}$ and ${ }^{27} \mathrm{Al}$ in framework aluminosilicates, $\delta_{i s o}$ decrases systematically with increasing mean angle. The 3QMAS datia fall close to a linc previously fitted 1.o clata from ordered phases, confirming the accuracy of the now dala and of the sile assignments. An earlier fit that included data for disordered mincrals as woll agrees corn more closely with the anorthite data. This agrement may be fortuitous. in that bond angle calculations for disordered crystals arre based on avorage long range structure, and thus may be distorted by the lack of data on truc local bouding geometry. 


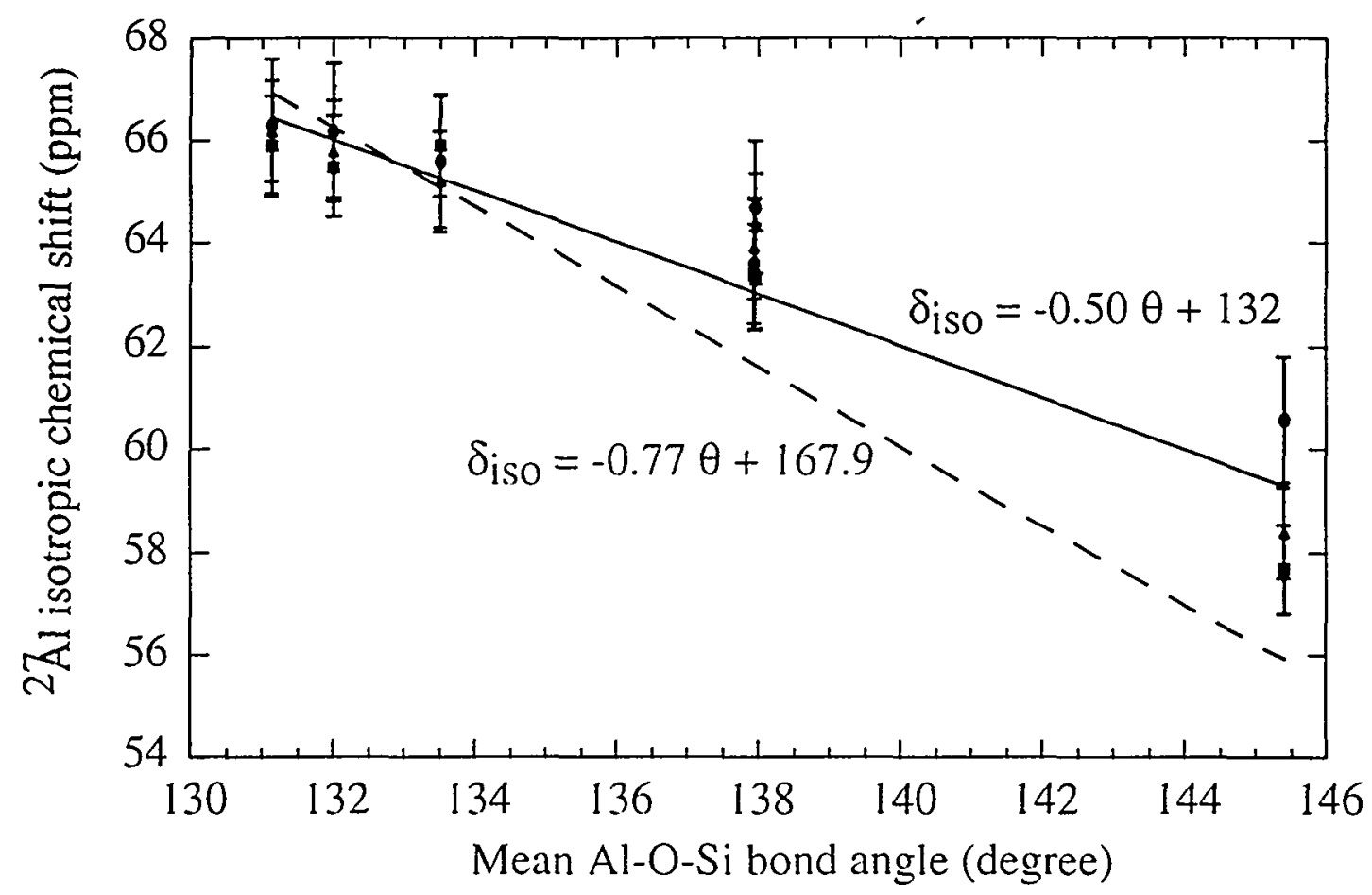

Figure 4.7: Isotropic chemical shifts for anorthile, derived from 3QMAS data, plotted against the mean Si-O-Al angle $(0)$ at cach site. Only six sites are plotted, as data for remaining two are not well constrained by the spectra. Solid circles: results from simulations of slices in 11.7 T spectrum; solid triangles: results from 2D peak positions at 11.7 ' $\mathrm{l}$; solid scuares: results from 2]) peak positions al $9.4 \mathrm{~T}$. Solid line is a fit to data for a variety of almminosilicales (both ordered and disordered) with $\delta=-0.500+132$ : dastod linc is a fir to dala for ordered structures only, with $\delta=$ $-0.770+167.9$.

\subsubsection{Anorthite ( $\left.\mathrm{CaAl}_{2} \mathrm{Si}_{2} \mathrm{O}_{8}\right)$ Glass}

The 3QMASS spertmun for llac glass of anorthile composition is shown in Figure 4.S. As expected from the MAS sperdrum, it is broad and umresolved. The peak maximum and fhe conter of mass are shiffed by roughly 5 ppm from those of the crystal in both dimensions. suggesting a decrease in the moan chemical shift and/or an increase in the mean ('Q 'The much greater oreall width is not surprising in light of the disorder in the glass.

In MAS spectra of glasses in which 211 is expectod to be four-coordinated by oxygen (as in this composition). Here is ofien significant spectral intensity in the 
region of isotropic chemical shifts for five- and six-coordinated Al. In the absence of clear, discrete peaks in such spectra, it. is generally assumed that such low-frequency tails are the result of second-order quadrupolar broadening. (This is supported by the narrowness of satellite transition sidebands in MAS spectra.) The 2D 3QMAS spectrum confirms this conclusion strongly: in comparison with clearly separated features for $\mathrm{AlO}_{5}$ and $\mathrm{AlO}_{6}$ groups as secn in the glass described next (Figure 4.9), there is no detectable int.nsity at these positions in the $\mathrm{CaAl}_{2} \mathrm{Si}_{2} \mathrm{O}_{8}$ glass. On the other hand, the 2D shapes of 1 lic $\mathrm{AlO}_{\mathrm{I}}$ peaks in both glasses are surprisingly similar, perhaps suggesting similar ranges of $\delta_{i s o}$ and $C_{Q}$.

\subsubsection{Magnesium Aluminoborate Glass}

This material was chosen because it contains sub-equal concentrations of four, five-, and six-coordinated $\mathrm{Al}$, which are clearly seen as partially resolved peaks in ${ }^{27} \mathrm{Al}$ MAS NMR spectra. The 3QMAS spectrum is shown in Figure 4.9, and has three well-separated peaks that can be assigned to the three coordinations. The lack of significant overlap of the 2D praks indicates that this approach may be very useful for detecting (or excluding) the presence of relatively small concentrations of the higher coordination states, whose prescuce is likely to be ambiguous in MAS spectra. Estimates of isotropic chomical shifis and quadrupolar products $P_{Q}$ for these peaks can be made by moasuring the positions of the peak maxima in both dimensions as

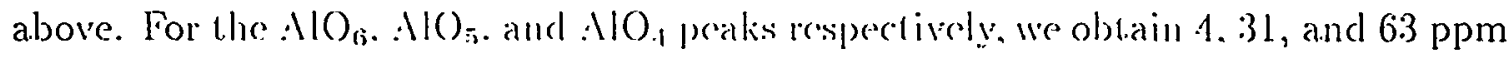
for $\delta_{i s o}$ and 2,3 , and $5.111 \%$ for ('Q. 'These result.s are complicated by the likelihood of overlap of signal from sitcs with varying parametcrs within each major peak, and uncertainties are at least 2 ppon and 0.5 to 1.0 MIL\%. The relative population of the above three environments obtained from the total projection of the 2D spectrum is about 1: $2: 6$. As shown before for the kyanite sample, however, the triple quantum 


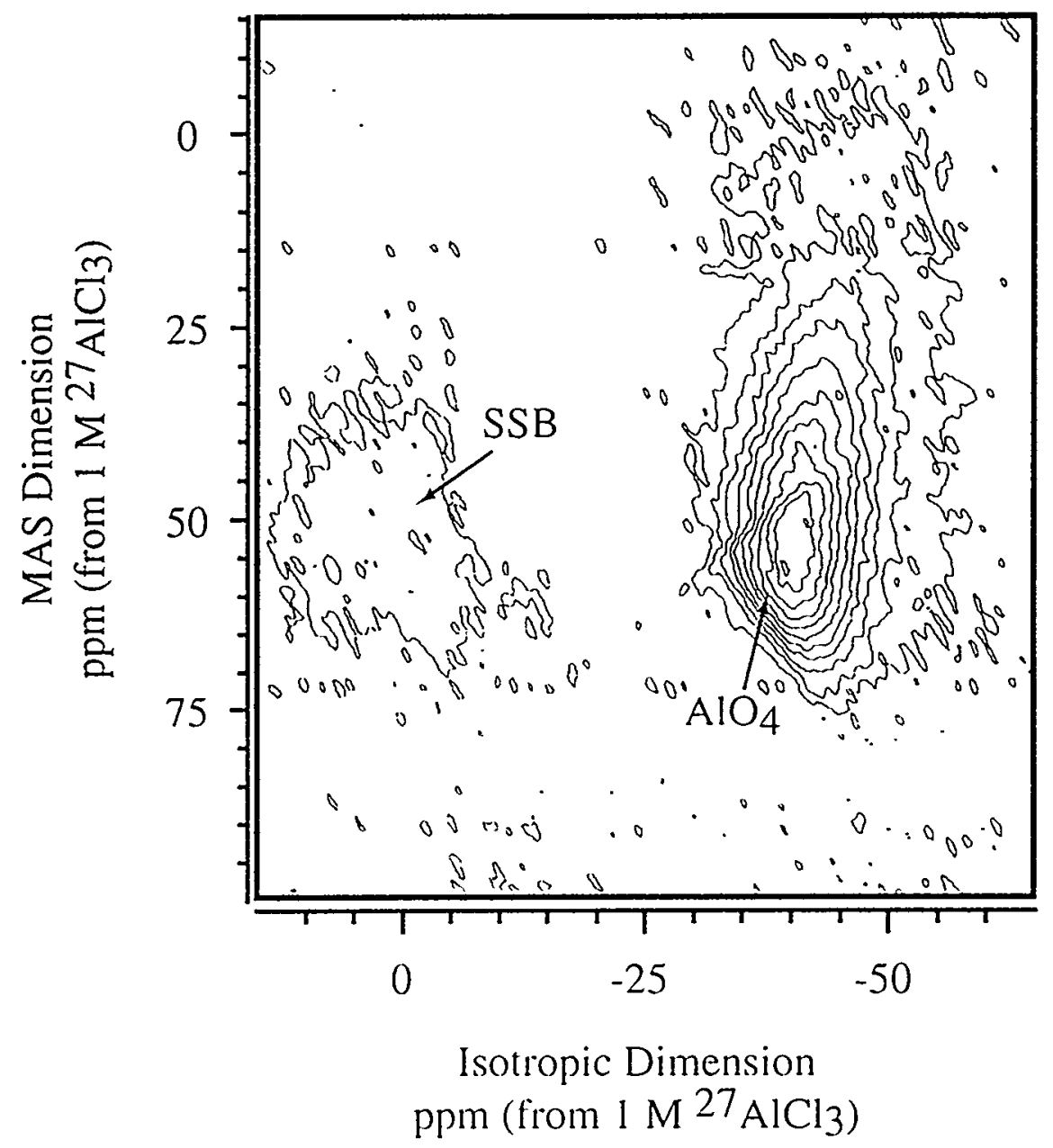

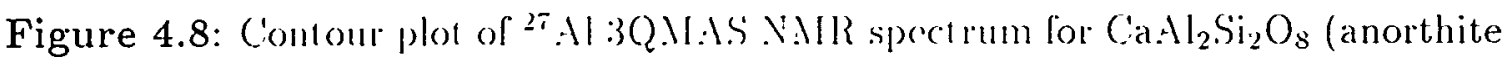

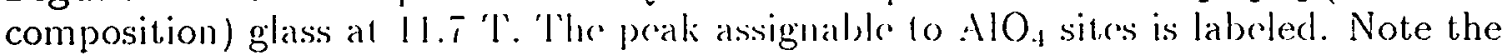

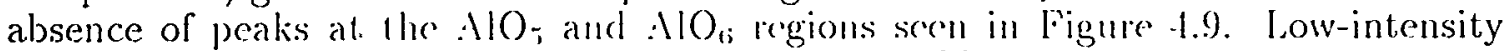
feature to left of main poak is a spinning sidcluand (SSBB). 
excitation efficiency for Al sil.es systemalically decreases with increasing $P_{Q}$. Thus, the intensity observed for the $\mathrm{AlO}_{4}$ peak is likely to be underestimated relative to the others.

The 2D spectrum is consistent with ranges of chemical shifts and coupling constants known from crystalline materials. $\mathrm{AlO}_{6}$ sites generally have $\delta_{i s o}$ between 1 and $15 \mathrm{ppm}$ and $C_{Q}$ between 1 and $10 \mathrm{MHz}$. This would result in 3QMAS peak positions of -1 to $-30 \mathrm{ppm}$ in the isotropic dimension and -50 to $12 \mathrm{ppm}$ in the MAS dimension. Values of $\delta_{i s o}$ for $\mathrm{AlO}_{5}$ sites lypically fall between 30 and $40 \mathrm{ppm}$, with $C_{Q}$ between 3 and $10 \mathrm{MHz}$, giving 3 QMAS peak positions ranging from -18 to $-40 \mathrm{ppm}$ in the isotropic dimension and -30 1.0 $30 \mathrm{p} p \mathrm{pm}$ in the MAS dimension. Finally, $\mathrm{AlO}_{4}$ sites typically have $\delta_{i s o}$ between 5.5 and $88 \mathrm{ppm}$ and $C_{Q}$ between 1 and $10 \mathrm{MHz}$, resulting in 3QMAS peak positions from -30 to $-60 \mathrm{ppm}$ in the isotropic dimension and 0 to S0 ppm in the MAS climension. Note that. in Figure 1.9, each of the labeled peaks falls neatly in the center of the corresponding regions. For the $\mathrm{AlO}_{4}$ peak we also fitted slices along the $\omega_{2}$ dimension, as was clone for the crystalline phases. Again, for a disordered material result.s from this procedure are non-unique because each slice contains unresolved intensily from sites with ranges in chemical shift and $C_{Q}$. However, this approach does give some estimates of the range of parameters present, about 62 to $7.5 \mathrm{ppm}$ for $\delta_{\text {iso }}$ and 1 10 $6.5 .5 \mathrm{.HH}$, for $C_{Q}$.

\subsubsection{Goosecreekite}

Goosecreekite is a mal.ural zcolite whose structure is still somewhat controversial. Earlier studies showed that hore is only one tet rahedral aluminum site in this zeolite. A recent refinement of the slructure. however, showed that two slightly different aluminum sites exist. The MAS spectrum of this sample shows however, only one peak. In this respect. MQQMAS would be an ideal technique to resolve this structural 


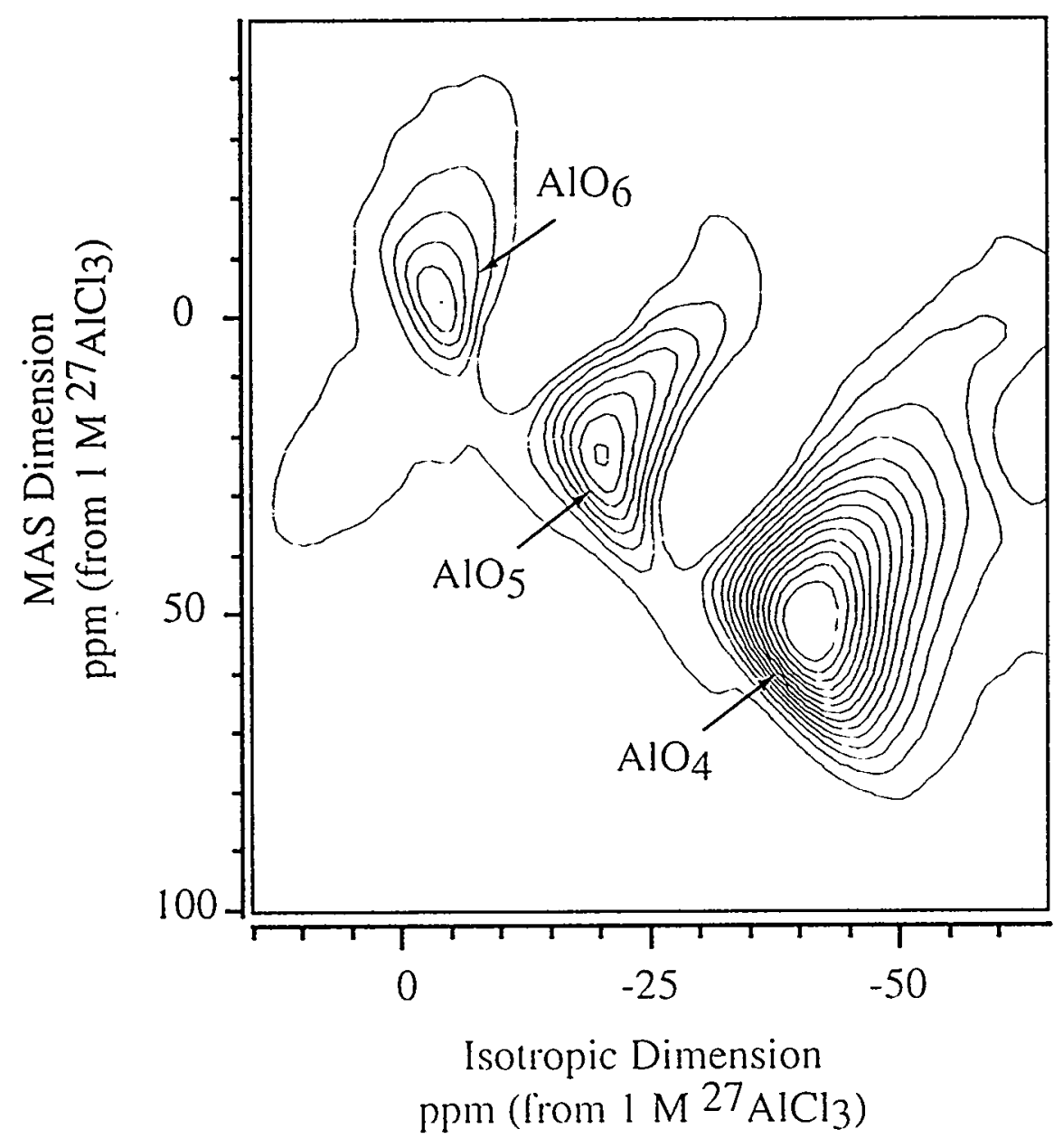

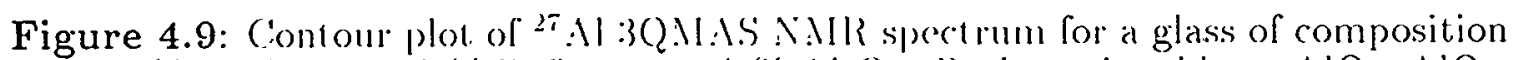

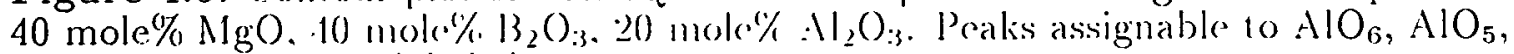
and $\mathrm{AlO}_{4}$ groups are labeled. 
problem.

Figure 4.10a shows the ${ }^{27} \mathrm{Al}$ 3QMAS spectrum of goosecreekite taken at $11.7 \mathrm{~T}$. The 1D 3QMAS spectrum has only one Gaussian peak (1ppm, FWHM), which is narrower than the MAS spectrum (5ppm, FW'HM). The two-dimensional spectrum has already resolved some structures. As one can see, the low frequency side of the isotropic dimension corresponds to a larger quadrupolar coupling constant, and thus a broader MAS dimension. Even though the spectrum can be interpreted as two overlapping peaks with slightly different chemical shifts and quadrupolar coupling constants, the assignment is not unique.

The 5QMAS spectrum in Figure 4.10b, however, shows clearly that there are two distinct aluminum sites in this \%olit.r. The olsserved frequency in MAS and 5QMAS can be described by

$$
\begin{aligned}
\delta^{M / A S} & =\delta_{i s O}+\delta_{i s O}^{2 Q} \\
\delta^{5 Q M A S} & =\frac{85}{37} \delta_{i S O}-\frac{50}{37} \delta_{i s O}^{2 Q} .
\end{aligned}
$$

Using the shifts in the single-quantum and multiple-quantum dimensions, isotropic shifts and quadrupolar coupling consiant.s for both siles can be extracted, using the

\begin{tabular}{|c|c|c|c|c|c|}
\hline site & $\delta \pi / .5(p p m)$ & $\left.\delta^{30.17 .15}(1) \mid m 1\right)$ & 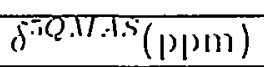 & $\delta_{i s o}(p p m)$ & $P_{Q}(\mathrm{MHz})$ \\
\hline 1 & 55.9 & -333.8 & $137 . \overline{5}$ & 58.4 & 2.7 \\
\hline 2 & 5.5 .2 & -:30.8 & 141.7 & 59.3 & 3.4 \\
\hline
\end{tabular}
strategy developed at. (he brginning of this chapter. The results are listed in Table 4.5.

Table 4.5: Isolropic chemical shifts and quadrupolar coupling products for goosecreekite.

The use of quint.uple-quant.um coherence to enhance the resolution of ${ }^{27} \mathrm{Al}$ spectra was well-studied by Amouroux in a series of aluminum-phosphate zeolites [79]. 


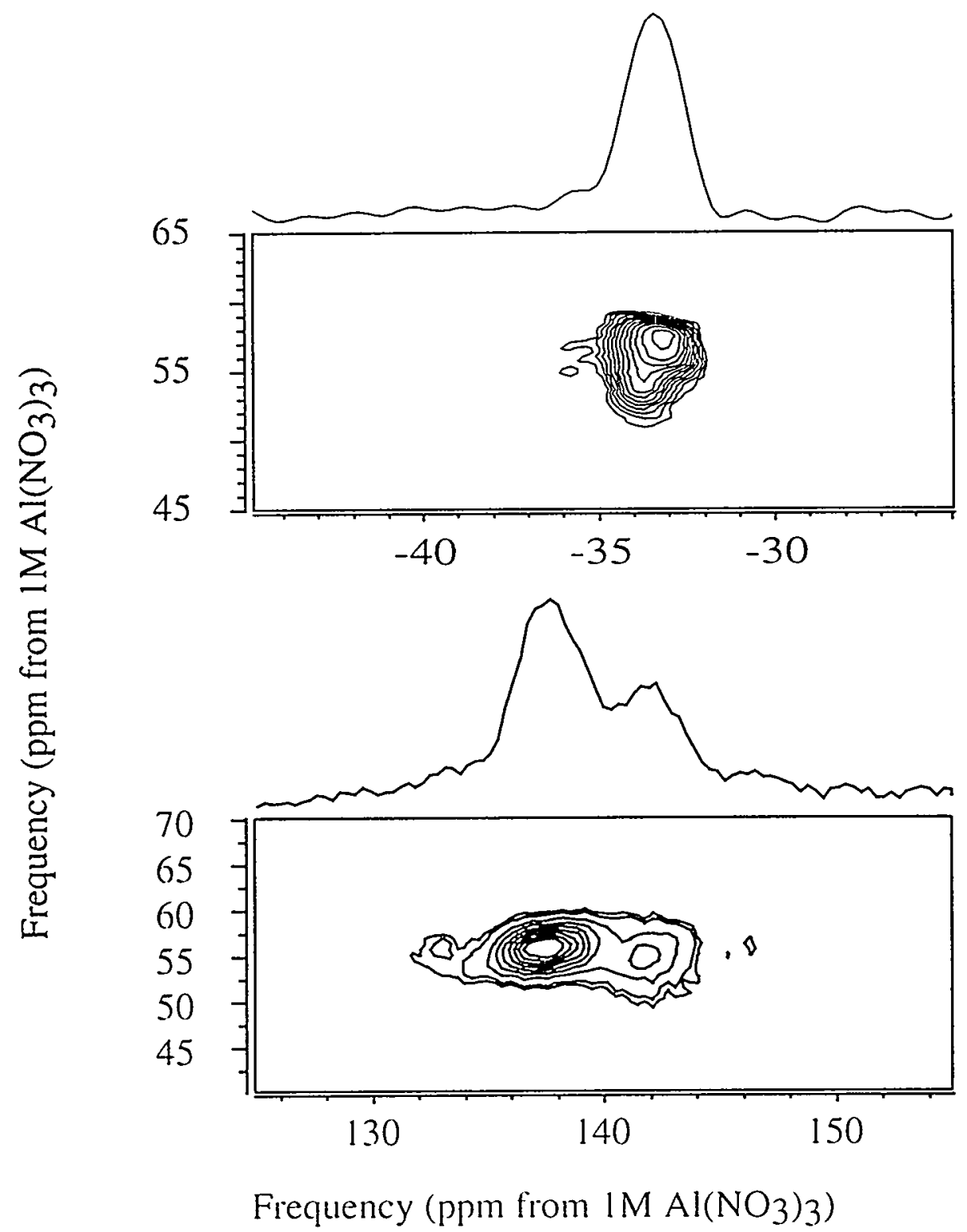

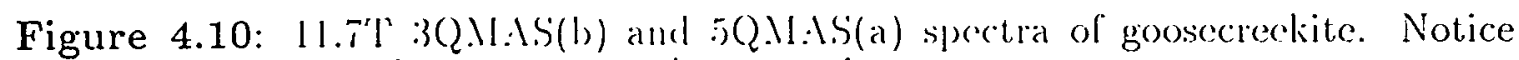
that even the $30 \mathrm{M} A \mathrm{~S}$ spect rmm resolves 1 wo siles. 
However, in all of the samples he studied and oir goosecreekite sample, the quadrupolar coupling constants are relatively small. The question of excitation for large coupling constants is a future problem in the utilization of this higher-order multiplequantum coherence.

\section{${ }^{17} \mathrm{O}$ 3QMAS}

Before concluding this clappler, I will include some initial results on the application of 3QMAS to the study of oxygen sites in silicate crystals. Oxygen is the most important element, in various types of materials and has been extensively studied by MAS and by DAS $[17,81,95,75,96.97 .100 .101,123,124,125]$. Because of its low resonance frequency and low natural abundance, isotopic enrichment is often needed in most of the studies.

Oxygen sites in silicate and aluminosilicalie materials are roughly classified into two types: the bridging and the non-bridging oxygens. The bridging oxygen connects two framework atoms (silicon or aluminum), whereas the non-bridging oxygen has charge compensating cations (often allaline or alkaline earth cations) as nearest neighbors. The cuadrupolar coupling constauls for the bridging oxygens range from 4-7MHz, which are larger than those for the non-bridging oxygens (2.5-3.5 MHz). This large difference in quadrupolar coupling const ants induces diflerent, quadrupolar shifts that the bridging and non-f)ridging oxygons arr at lass partially scparated in a DAS or MQMAS spectrum.

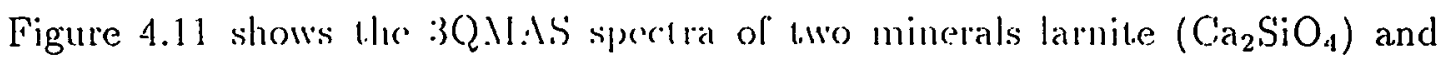
forsterite $\left(\mathrm{Ng}_{2} \mathrm{SiO}_{1}\right)$. ('orresponding 1$) \mathrm{ASS}$ spectra revealed 1 praks for larnite, and 3 sites for forsterite [75]. The 3QQMAS spectra of larnite gives only two resolved peaks. The three forsterite praks are however, all resolved. In both samples, only non-bridging oxygens exist so all of the sitcs are expected to be excited. For another 


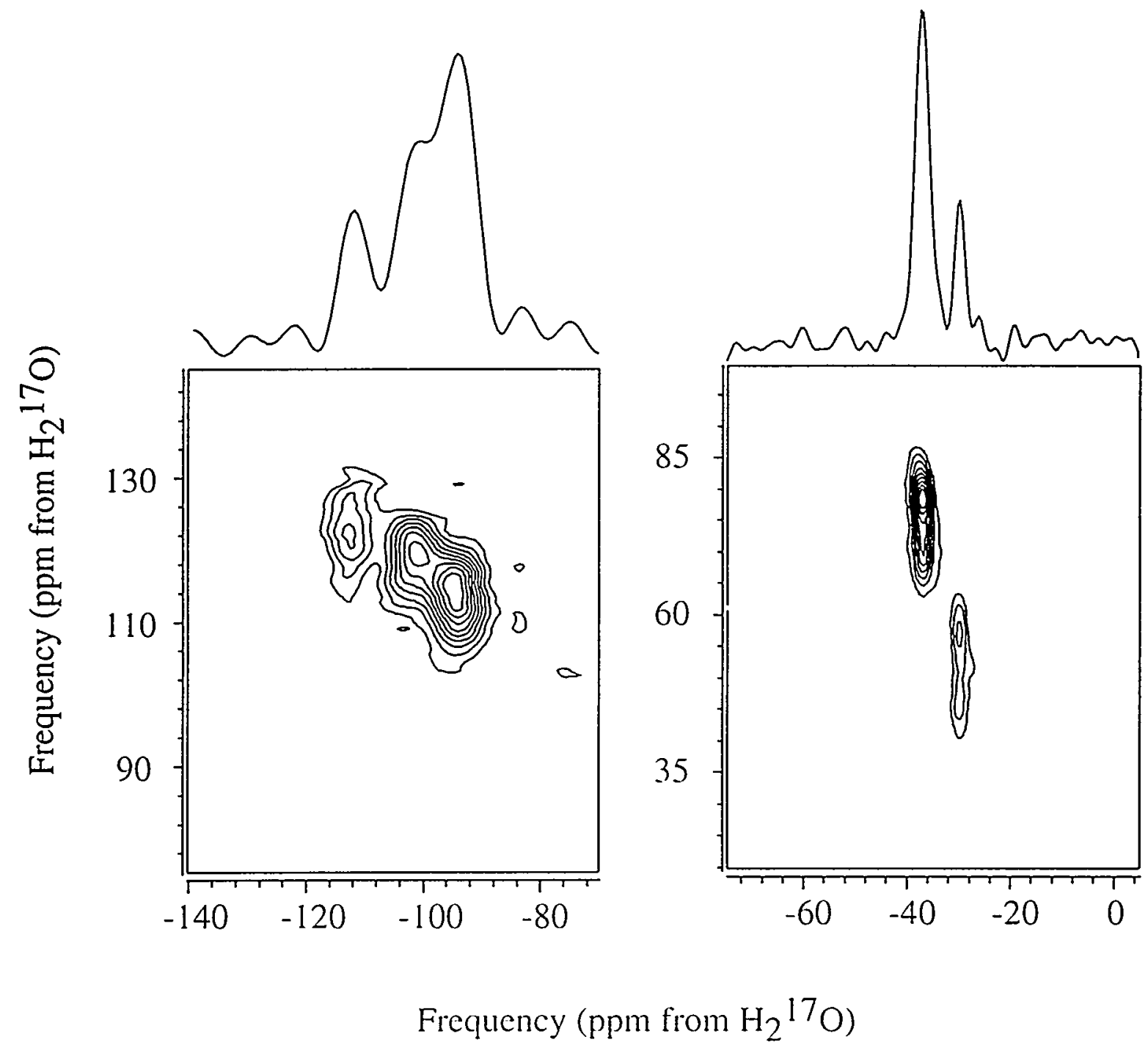

Figure 4.11: $\left.\left|1 . \mathrm{i}^{\prime}\right|^{17} \mathrm{C}\right) 3(2 . \mathrm{M}: \mathrm{AS}$ of larnite (Iefi) and forsterite (right). 
sample enstatite, whore bridging oxygen sites also exist, we were not able to excite the bridging oxygens using a relalively low power (30-40kHz). However, 3QMAS experiments on bridging oxygens have been performed in many groups, and their results suggest that such experiments are feasible when the enrichment level is high $(20-40 \%)$ and when the spin-latice relaxalion time is short (less than 1 second) $[56,116,126,127]$. More importantly, oxygen sites in Si-O-Si and Si-O-Al linkages are often well-separated. It is also interesting that when $T_{1}$ is short, 3QMAS actually gives better $\mathrm{S} / \mathrm{N}$ than DAS. Based on these proliminary works, it is quite promising to use 3QMAS for the sl.udy of a scrics of Icchnologically important materials, including multi-component oxides, zcolites and supported oxide catalysts.

\subsection{Conclusions}

Multiple-quantum magic-angle spinning (MLMAS) spectra can provide enhanced resolution for ${ }^{27} \mathrm{Al}$ in aluminosilicalce and aluminale materials, both crystalline and amorphous, although resolution in the isolropic dimension may still be limited by disorder and other less well understood mechanisms of residual broadening. Additional information on NMR parameters may be olfainable because the two dimensional spectra provide some scparation of chemical shilt, and cuadrupolar effects, both from simple peak position data and from lithing of XIS poak shapes in slices of the spectra. MQMAS signal can be ohtaincel cren from siles will guadrupolar coupling constants as large as $9 \mathrm{M} 11 \%$, but intonsily is systomalically roduced with increasing $C_{Q}$. We have derived new data for isol ropic chemical shifts for five or six of the eight sites in crystalline anorthite. which agrec reasomably woll with previous correlation with structure. In glasses, the separation between peaks for Al in different coordination states is excellent, and provides a now and sensitive lest for the presence of $\mathrm{AlO}_{5}$ and $\mathrm{AlO}_{6}$ sites in glasses dominaled $\mathrm{by}: \mathrm{AlO}, \mathrm{f}$. allhough absolute quantification may 
remain difficult. 


\section{Chapter 5}

\section{Correlation Spectroscopy with MQMAS}

Up to now, we have only considered the NMR spectra under single-spin interactions (chemical shift and/or quadrupolar interactions). To interpret NMR spectra of networks of spins, connectivity: among different spins is to be established. Heteronuclear correlation ( $H \mathrm{~S}^{\prime} \Gamma(\mathrm{O})$ ) as a means of mapping out the spin topology, is proved powerful to correlate the chemical shifts of directily coupled spins and elucidate the structure of heteronuclcar coupling networks [5]. HETCOR in the solid-state has been limited to pairs of spin- $\frac{1}{2}$ nuclei in the past, clue too the lack of high-resolution for quadrupolar nuclei. With the development of DAS and MQMAS, we demonstrate in this chapter that, true high-resolution IIETCOR spectra are equally obtainable for quadrupolar nuclei.

\subsection{Heteronuclear Correlation (HETCOR)}

Heteronuclear corrclation spectroscopy is a routine method in liquid-state NMR and represents only a special cxperiment anong a series of two-dimensional correlation methods [5, 8]. The key of the experiment is colierence transfer between two types of coupled horcrospins. In wrakly compled systoms, coherence transfer between two spins occurs only if llecre is a nom-ramishing dipolar-or J-coupling between the spin pair. Since the magnitude of dipolar and J-couplings is inversely proportional to the third power of we distance betwern the fwo spins, only those spins that are in spatial proximily show significant. couplings and induce colerence transfer. As a result, the appearance of cross-peaks in 2 D HETCOR spectra serves as a proof of spatial proximity betwern the coupling partners. The spectrum is thus a visu- 
alization of the topology of the spin system in a direct and informative way. This detailed information about the spin system is often essential for the determination of the structure of large molecules [128] and complicated materials [129].

It is worth describing cross-polarization $((: P)[13,130]$ here before we go into any details about HETCOOR. (ross-polarization is the most widely used technique in the solid-state to achicvo hetcronuclear colicrence transfer and signal cnhancement. Dipolar coupling, whose magnitude of ten excecels that of the .J-coupling by an order of magnitude, is the basis of the coherence transfer process. The simplest CP scheme, as shown in Pigure 5.l. starts by applying a $\frac{\pi}{2}$ pulse along the rotating frame $y$ -

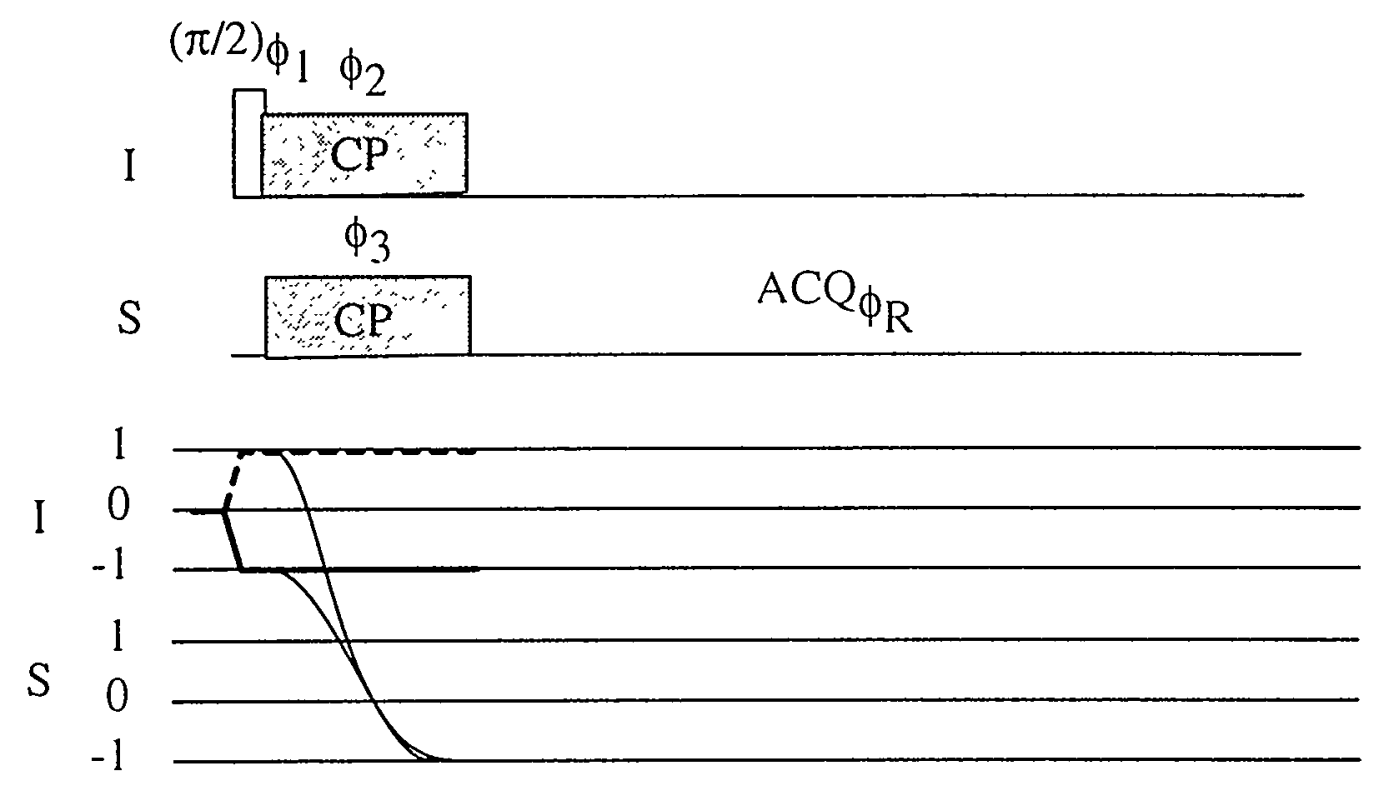

Figure 5.1: ('ross-Polatizalion lockern lwo spins I and S.

axis on one type of the spins (ustlally the more abundant spin type that serves as magnelization source and is lormod as l-spin). This pulse gemorates single-quantum coherence on the I-spins, which is subsegurotly locked along the rotating frame $x$ axis by applying a long pulse along llac $x$-axis. If a Iong pulse is also applied to the S-spins (the less abundant spin type that borrows magnetization from I-spins), and 
the magnitude of the RF field fullfills the Ilartmann-Hahn condition

$$
\gamma_{1} B_{1 I}=\gamma_{s} B_{1 S}
$$

the precession frequencies of the two types of spins are equal and the flip-flop terms in the dipolar Hamiltonian are now zero-energy process. This greatly enhances the energy transfer process beliveen two types of spins and the net result is often that the rare spin (S-spin) magnetization is amplified significantly. For instance, When cross-polarization (CP) is applied to a ${ }^{1} \mathrm{II}^{13} \mathrm{C}^{\mathrm{C}}$ system, a gain of a factor of $3-4$ in ${ }^{13} \mathrm{C}$ polarization can be achieved. The signal to noise $(\mathrm{S} / \mathrm{N})$ ratio is often boosted by more than an order of magnilude, since ${ }^{1} \mathrm{H}$ has shorter $T_{1}$ thand ${ }^{13} \mathrm{C}$ that fast repetition can be used wilh ('P. Currently. cross-polarization magic-angle spinning (CPMAS), which combines cross-polarizalion with magic-angle spinning, is the single most routine experiment performed in most. solid-state NMR laboralories.

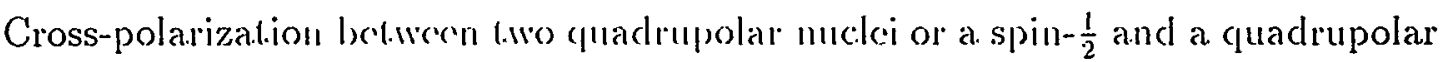
nuclei suffers a number of difficulties. First, the rotating frame Hamiltonian is dominated by the large first-order quadrupolar interaction, which is highly anisotropic that the quadrupolar coupling constant depends on the crystalline orientations.

$$
C_{Q}^{\epsilon S S}=\frac{C_{Q}}{2}\left(3 \cos ^{2} 33^{Q}-1+\eta_{Q} \sin ^{2} \beta^{Q} \cos 2 \alpha^{Q}\right)
$$

Here, $C_{Q}^{e f f}$ is the effective quadrupolar coupling constant and $\left(\alpha^{Q}, \beta^{Q}, \gamma^{Q}\right)$ are the Euler angles between the guadrupolar principal axis frame (PAS) and the lab frame. The differences in the chuadmpolar coupling constants complicate the HartmannHahn matching condition for cross-polarizalion [69. 70$]$. For instance, when $C_{Q}^{e f f} \ll$ $\omega_{1}$, the Hartmann-Hahu conclition is the same as that of a spin- $\frac{1}{2}$ pair.

$$
i_{1} B_{11}=\gamma_{i=} B_{1,5}
$$

However, when $C_{Q}^{c f S} \gg \omega_{1}$, the Ilarlmann-IIahn condition needs to be modified to include a constant. factor dependent on the spin cliantum number of the quadrupolar 
nucleus.

$$
\gamma_{11} B_{11}=\left(S+\frac{1}{2}\right) \gamma_{s} B_{1 s}
$$

In a powder sample, it is often the case that neither of the above two conditions are fulfilled and the cllective (puadrupolar coupling constant $C_{Q}^{e f f}$ is comparable to the RF strength $\left(\omega_{1}\right)$. The spin dynamics is far more complicated (see the discussions in chapter 3 on spin locking) that no single inluilive formulus exists for the whole sample.

The matching condition in a rolating sample is further complicated by two facts. First, the dipolat interactions that mediate the C.P transfer are averaged to zero over a rotor period (the instantancous dipolar coupling, instead, still exists and induces coherence lanansfer), reducing the cfficioncy of cross-polarization (CP). Second, the effective coupling const ant for a cuadrupolar spin is no longer constant and fluctuates periodically. As a result. Hore exists no migue matching condition that all of the spins with different crestalline oriontalions fullill. 'This suggests that ('P spectra involving quadrupolar muclei are often not quantilative. Nerentheless, the fact that only spin pairs with non-marnishing dipolar conplings give rise to coherence transfer

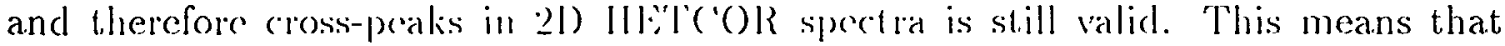
HETCOR exporiment berwern quadrupolar and spin- $\frac{1}{2}$ muclei rould in principle, provides similar information on the spin compling nowork compared to the correlation for spin- $\frac{1}{2}$ pairs.

A simple HIEl' 'OR sepuence is ronstructed by incorporating the (YP sequence with a simple onc pulse experiment on S-spins. As shown in Figure 5.2 , a $\frac{\pi}{2}$ pulse applied to the I-spuns brings t.le I-spin magnetization into the rey plane of the laboratory frame. The magnetization volves under lhe l-spin Hamiltonian for a variable time $t_{1}$, during which llac l-spin resonanes ferpurnocies are concoded into the resultant magnetization. Throngh cross-polarization. This magneti\%ation on the I-spin is 

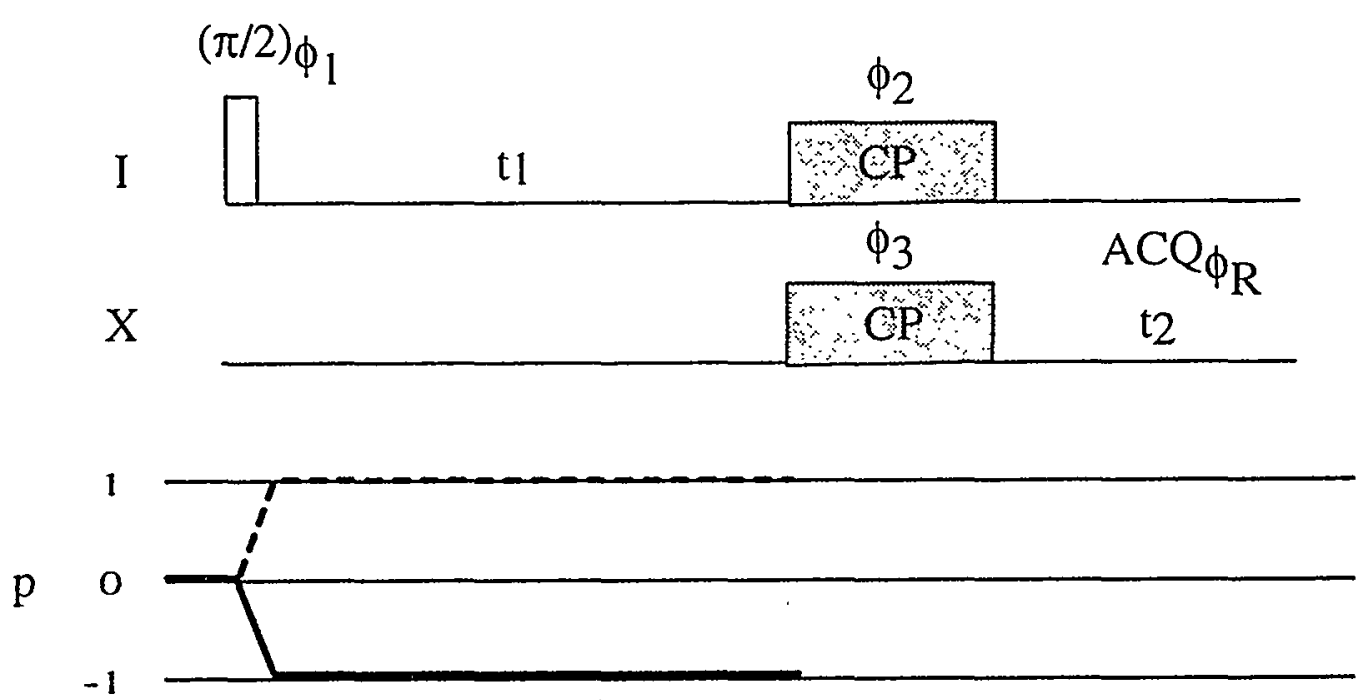

Figure 5.2: Pulse sequence and coherence pathway for a traditional HETCOR experiment. Notice that only the coherence pathway on I spin is shown.

transferred into S-spin magnetizalion. Which continues to evolve under the S-spin Hamiltonian and is recorded with quadrature dedection. The lwo frequency domains after two-dimensional louricr lansformation are the I- and S-spin chemical shifts, with cross-pealis appeating between the resonancess of dipolar coupled heteronuclear spins. Magic-angle spinning is usually applied during the whole experiment to enhance the spectral resolution in both dimensions.

\subsection{HETCOR with Quadrupoles}

Figure 5.3 is an samplo of regular wo-dimonsional IIETCOR spectrum between a spin- $\frac{1}{2}$ and a quadrupolar mucleus. The ${ }^{23} \mathrm{Na} /{ }^{31} \mathrm{P}$ HETC:OR spectrum is taken on sodium trimotaphosphate $\left(\mathrm{N}_{a_{3}} \mathrm{P}_{3} \mathrm{O}_{9}\right)$. Which was prepared by heating $\mathrm{NaH}_{2} \mathrm{PO}_{4}$ at $550^{\circ}$ for 3 hours and slowly cooling the sample down lo room lomperature [4:3]. X-ray diffraction (XRD) show's that lhe samplo is woll-crystallized and of the correct phase. MAS NMR spectra on ${ }^{23} \mathrm{Aa}$ and " $" \mathrm{p}$ confirm the conclusion that there are two distinct types of ${ }^{23} \mathrm{~N}$ a and ${ }^{31} \mathrm{P}$ 'sitc's in lhis compound. 'The two phosphorus peaks, -18.7 and 


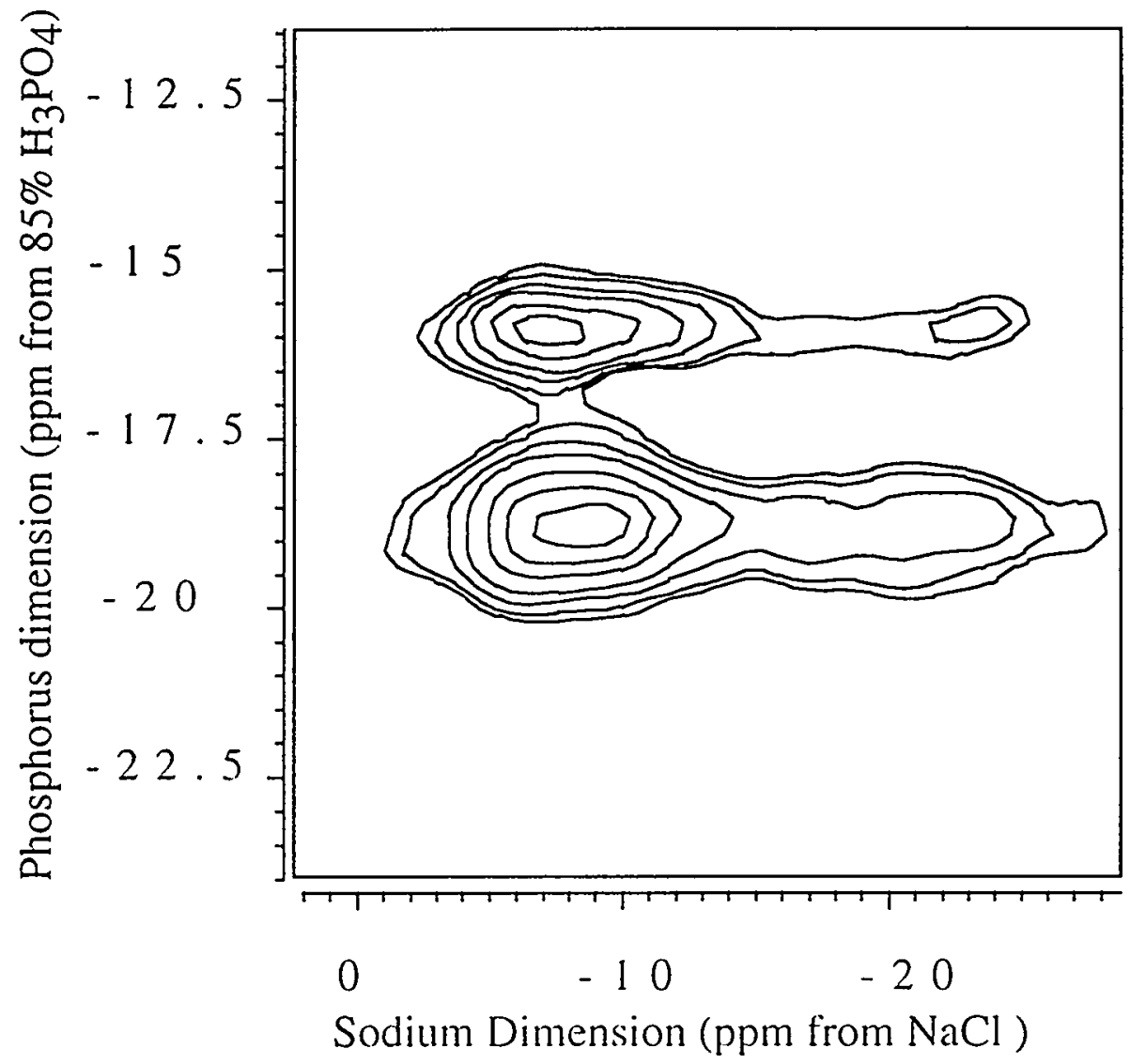

Figure 5.3: A convontional mo-dimonsional ${ }^{23} \mathrm{Na}^{31} \mathrm{P}$ HE.TCOR spectrum of $\mathrm{Na}_{3} \mathrm{P}_{3} \mathrm{O}_{9}$ collected at $11 . T^{T}$ '. 'The sample is spimning at. $5 \mathrm{kHz}$ and the contact time is $5 \mathrm{msec}$.

$-15.5 \mathrm{ppm}$ away from a $\$ 5 \% \%, \mathrm{H}_{33} \mathrm{P} \mathrm{P}_{.}$standard $(0.0 \mathrm{ppm})$, are the general and mirror

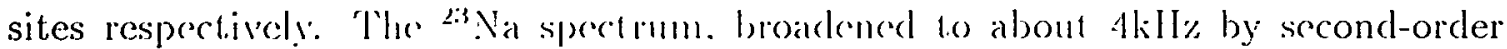
quadrupolar intoraction. (an lx decomoluted into fwo quadrupolar powder patterns, with isolropic chemical shifts at -6.2 and -2.2.1 ppm with respere to solid $\mathrm{NaCl}$ at 0.0 ppm.

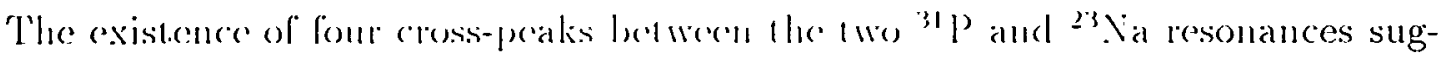
gests that each ${ }^{31} \mathrm{P}$ site is mall to both ${ }^{23} \mathrm{Na}$ siles. Similarly, each ${ }^{23} \mathrm{Na}$ site is also near to both ${ }^{31} \mathrm{P}$ sites. 'This qualitatively agress to the crystal structure of $\mathrm{Na}_{3} \mathrm{P}_{3} \mathrm{O}_{9}$ that all of the sorlium and phosphorus positions are intercomected (through oxygen atoms). 
Notice that the HEIC:OR experiment is performed by transferring coherence from the lower gyromagnetic ratio quadrupolar nucleus $\left({ }^{23} \mathrm{Na}\right)$ to the higher gyromagnetic ratio spin- $\frac{1}{2}$ nucleus $\left({ }^{31} \mathrm{P}\right)$. This is the reversed case of most CP experiments. For

cross-polarization between spin- $\frac{1}{2}$ and quadrupolar nuclei, unless ${ }^{1} \mathrm{H}$ or ${ }^{19} \mathrm{~F}$ is involved or the quadrupolar nucleus is of very low abundance, CP from spin- $\frac{1}{2}$ nucleus to quadrupolar nucleus does not culance the signal to noise ratio $[42,70,131,132]$. Two reasons account for this anomaly: (1), C.P between quadrupolar and spin- $\frac{1}{2}$ nuclei is often inefficiênt; (2), spin- $\frac{1}{2}$ nuclei often have long spin-lattice relaxation times $\left(T_{1}\right)$ which preclude rapid signal averaging. Therefore CP is better used as a spectral editing method, rather than a signal-enhancing technique. On the contrary, like in the current casc. (!P from quadrupolar spins enhances sensitivity of the spin- $\frac{1}{2}$ nucleus.

Due to the complex spin dynamics during cross-polarization, it is not easy to retrieve exact distance information from the IIPT':OR exporiment. Also, the resolution in the quadrupolar dimension is low. The resolution problem is less severe for $\mathrm{Na}_{3} \mathrm{P}_{3} \mathrm{O}_{9}$, where the lwo sorlium sites are separated by lheir chemical shifts. For more interesting matcrials with complex structures and overlapping resonances, it is important to have liquid-like resolution in the quadrupolar dimension. As shown in the next section, high-resolution is achicrable for cluadrupoles in both dimensions of a HETCOR spectrum, whon DAS or MQMAS is applied to the regular HETCOR experiment.

\subsection{High-Resolution HETCOR}

True high-resolution correlation between quadrupolar and spin- $\frac{1}{2}$ nuclei can be achieved by correlating the dyuamir-angle spinning (DAS) or multiple-cuantum magicangle spinning (MQMAS) spertrum of a guadrupolal muclous with the magic-angle 


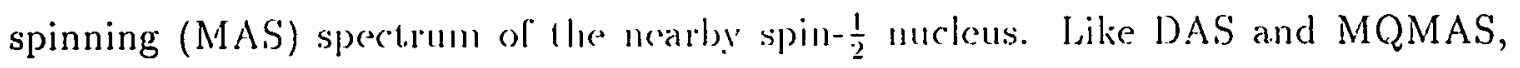
both approaches reconstruct the isotropic quadrupolar dimension by breaking up the $t_{1}$ evolution time into two part.s. The anisotropic resonance frequency of each spin is rendered to have opposilc signs cluring the two separated $t_{1}$ time periods of the evolution. When the ratio belween the lwo limes are well-selected, anisotropies arising from CSA and the scoond-orelor cuadrupolar interaction are removed and an echo is formed at the end of $I_{1}$ crolution.

In Figure 5.4, we compare lhe experimemlal schemes and coherence pathways of DAS/HETCOR and MQQMAS/HEI'OR. For DAS/IIEIC:OR, a DAS experiment is performed first on ${ }^{2: 3} \mathrm{Na}$ before the sodium magnetizalion is transferred to ${ }^{31} \mathrm{P}$ through cross-polarization. The experiment proposed by Jarvi ot al chose the $\left(79.19^{\circ}, 37.38^{\circ}\right)$ angle pair with $k=1$. 'The samplestals at $79.19^{\circ}$ and $37.35^{\circ}$ for an equal amount of time $\left(\frac{t_{1}}{2}\right)$, which creales a DAS echo at. Hhe ond of the $t_{1}$ period due to the refocusing of the second-order quadrupolar interaction. Cross-polarization is then performed at $0^{\circ}$ to maximize hac (!) efliciency. This results in a second rotor axis reorientation that brings the spinner axis to the direction of the static fickl. After CP, another reorientation of the rotor axis is merded to allow dalla acquisition al the magic-angle. Including the final hop during recerele delay that lorings llor spinner axis back to

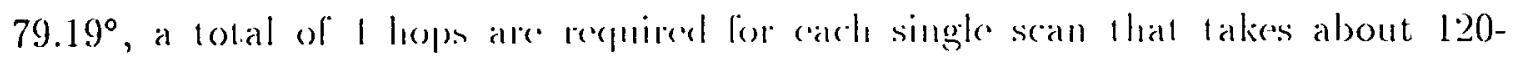

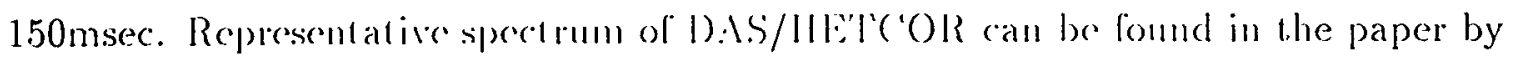

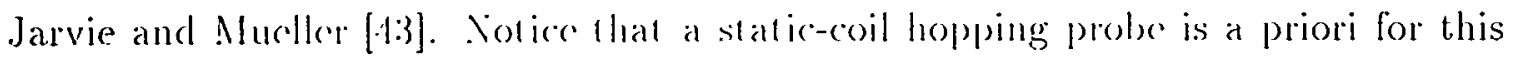
type of experiment, since pulsing at $0^{\circ}$ is mereded.

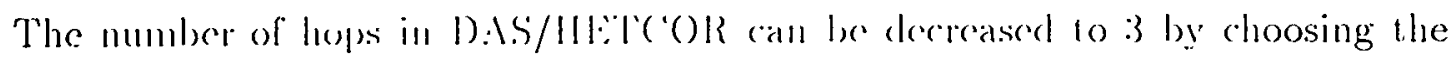
$k=5$ DAS angle pair. This howerer, still reculures a hopping DAS probe. The MQMAS/IIETC:OR experimont. Which does not involve reorientation of spinner axis, relieves the recuirement of a static-coil hopping DAS probe. It starts by excit- 
(a) $\quad(\pi / 2)_{\emptyset_{1}} \quad(\pi / 2)_{\phi_{2}}(\pi / 2)_{\emptyset_{3}} \quad(\pi / 2)_{\varnothing 4}(\pi / 2)_{\phi_{5}}$ ${ }^{23} \mathrm{Na} \prod \mathrm{t} 1 /(1+\mathrm{k}) \prod$ hop $\prod \mathrm{kt} 1 /(1+\mathrm{k}) \prod$ hop $\square \mathrm{B}$ $(\pi / 2)_{\emptyset_{6}}(\pi / 2)_{\emptyset_{7}}$

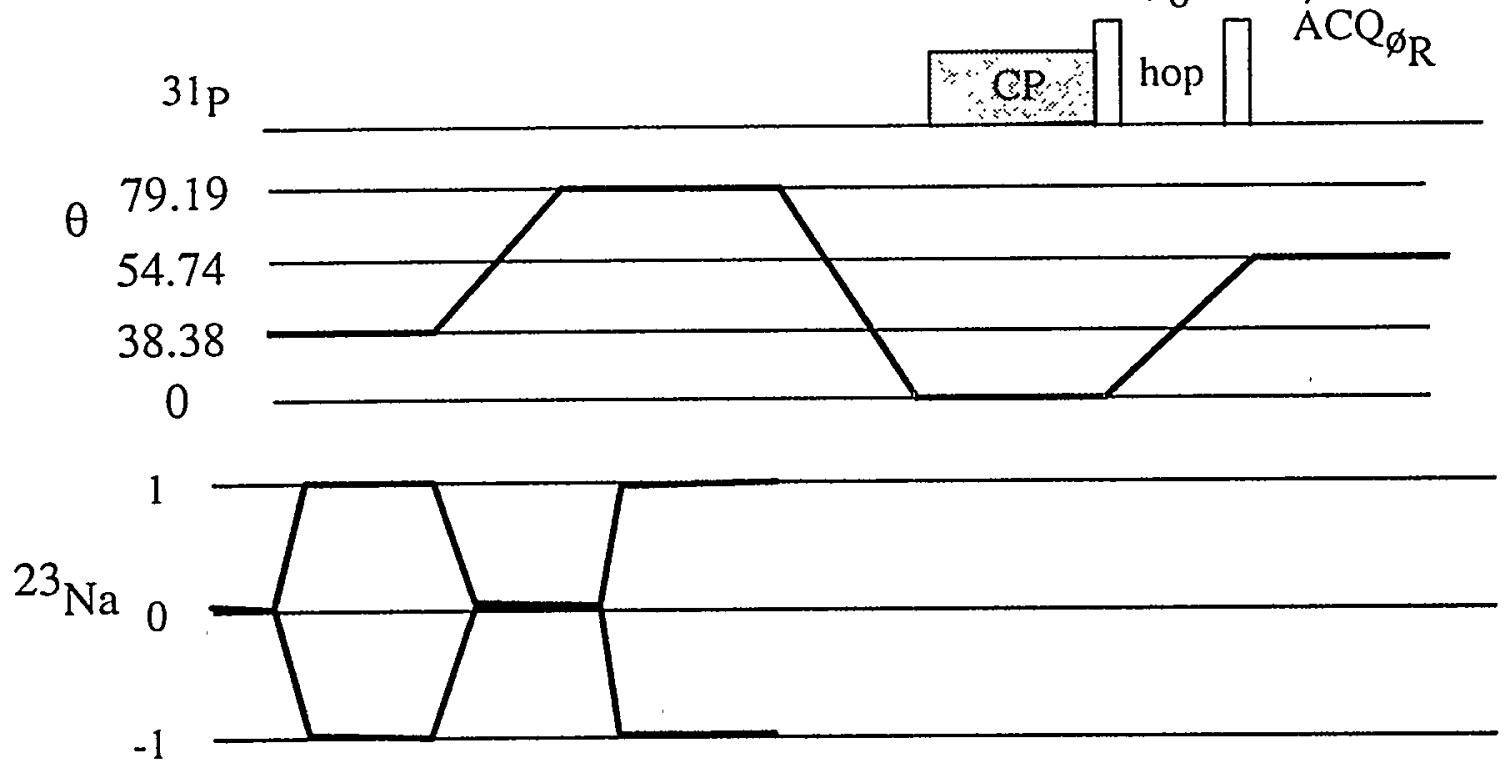

(b)
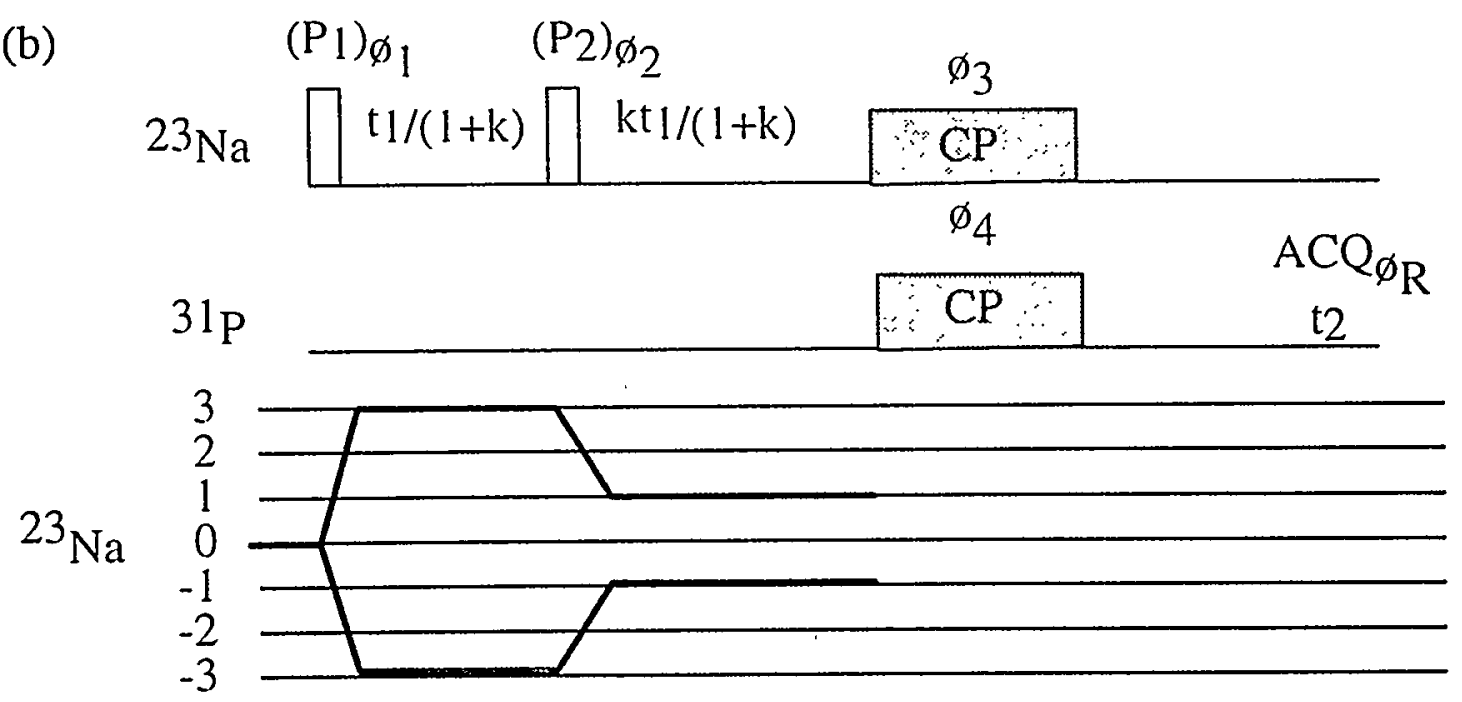

Figure 5.4: Comparison of DAS/HET'(OR and MQMAS/HETCOR pulse sequences and colisernce transicr pathway's. 
ing the triple-quantum coherence on ${ }^{2: 3} \mathrm{Na}$. As described in earlicr chapters, the excitation is most efficionly porformed by applying a single strong RF pulse near to the sodium resonance frequency. The triple-quantum coherence is then allowed to evolve for $\frac{t_{1}}{1+k}$ and then another strong pulse (reconversion pulse) transfers the triple-quantum coherence to single-quantum coherence, which evolves for $\frac{k t_{1}}{1+k}$ before cross-polarization is donc at the magic-angle. The subsequent detection period is the same as in DAS/HEI':OR.

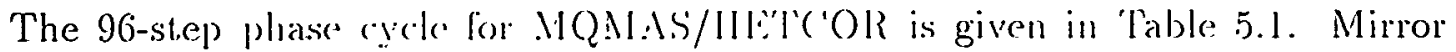
image coherence lausfor pallawas are maincel during the two separated $t_{1}$ period, leading to two-dimensional pureabsorption limeshapes. The phase cycle has incorporated CYYCLOPS and spin-10mporalure altcrnation to remove possible artifacts due to imperfections of hardware selling. A separate dataset that shifts the phase of $\phi_{3}$ by $90^{\circ}$ is needer to allow for purcalsorplion spectra.

\begin{tabular}{|c|c|c|c|c|c|c|c|c|c|c|c|c|}
\hline \multicolumn{13}{|c|}{ IQQMAS/IIL:L'COR cosinc } \\
\hline$\phi_{1}$ & $0^{\circ}$ & $60^{\circ}$ & $120^{\circ}$ & $180^{\circ}$ & $2-10^{\circ}$ & $300^{\circ}$ & & & & & & \\
\hline \multirow[t]{2}{*}{$\phi_{2}$} & $0^{\circ}$ & $0^{\circ}$ & $0^{\circ}$ & $0^{\circ}$ & $0^{\circ}$ & $0^{\circ}$ & $90^{\circ}$ & $90^{\circ}$ & $90^{\circ}$ & $90^{\circ}$ & $90^{\circ}$ & $90^{\circ}$ \\
\hline & $180^{\circ}$ & $180^{\circ}$ & $180^{\circ}$ & $180^{\circ}$ & $180^{\circ}$ & $\operatorname{lis} 0^{\circ}$ & $270^{\circ}$ & $270^{\circ}$ & $270^{\circ}$ & $270^{\circ}$ & $270^{\circ}$ & $270^{\circ}$ \\
\hline$\phi_{3}$ & \multicolumn{12}{|l|}{$0^{\circ}$} \\
\hline \multirow{8}{*}{$\phi_{4}$} & $0^{\circ}$ & $0^{\circ}$ & $0^{\circ}$ & ()$^{\circ}$ & $0^{\circ}$ & ()$^{\circ}$ & ()$^{\circ}$ & ()$^{\circ}$ & $0^{\circ}$ & $0^{\circ}$ & $0^{\circ}$ & $0^{\circ}$ \\
\hline & $0^{\circ}$ & $0^{\circ}$ & $0^{\circ}$ & ()$^{\circ}$ & $0^{\circ}$ & $0^{\circ}$ & $0^{\circ}$ & $0^{\circ}$ & $0^{\circ}$ & $0^{\circ}$ & $0^{\circ}$ & $0^{\circ}$ \\
\hline & $90^{\circ}$ & $90^{\circ}$ & $90^{\circ}$ & $90^{\circ}$ & () $0^{\circ}$ & $9)^{\circ}$ & $9\left(0^{\circ}\right.$ & $90^{\circ}$ & $90^{\circ}$ & $90^{\circ}$ & $90^{\circ}$ & $90^{\circ}$ \\
\hline & $90^{\circ}$ & $90^{\circ}$ & $90^{\circ}$ & $(9)^{\circ}$ & $90^{\circ}$ & 9()$^{\circ}$ & $9\left(0^{\circ}\right.$ & $90^{\circ}$ & $(9)^{\circ}$ & $90^{\circ}$ & $90^{\circ}$ & $90^{\circ}$ \\
\hline & $180^{\circ}$ & $180^{\circ}$ & $180^{\circ}$ & $\ln (0)^{\circ}$ & $\operatorname{los}\left(0^{\circ}\right.$ & $\sin 0^{\circ}$ & $1 s 0^{\circ}$ & $\operatorname{ls} 0^{\circ}$ & $1 s 0^{\circ}$ & $180^{\circ}$ & $180^{\circ}$ & $180^{\circ}$ \\
\hline & $180^{\circ}$ & $180^{\circ}$ & $180^{\circ}$ & $180^{\circ}$ & $180^{\circ}$ & $\ln \left(0^{\circ}\right.$ & $180^{\circ}$ & $\operatorname{ls}\left(0^{\circ}\right.$ & $1 \$ 0^{\circ}$ & $180^{\circ}$ & $180^{\circ}$ & $180^{\circ}$ \\
\hline & $270^{\circ}$ & . & $2 \pi 0^{\circ}$ & $2 \frac{-1}{1}()^{\circ}$ & $270^{\circ}$ & $2 \pi 0$ & - ${ }^{-1}\left(0^{\circ}\right.$ & $2 \pi 0^{\circ}$ & $2 \pi\left(0^{\circ}\right.$ & $270^{\circ}$ & $270^{\circ}$ & $270^{\circ}$ \\
\hline & $270^{\circ}$ & $270^{\circ}$ & $270^{\circ}$ & $270^{\circ}$ & $270^{\circ}$ & $270^{\circ}$ & $270^{\circ}$ & $270^{\circ}$ & $270^{\circ}$ & $270^{\circ}$ & $270^{\circ}$ & $270^{\circ}$ \\
\hline \multirow[t]{4}{*}{$\phi}$. & $0^{\circ}$ & $180^{\circ}$ & $0^{\circ}$ & $1 \leqslant 0^{\circ}$ & $0^{\circ}$ & $\sin \left(0^{\circ}\right.$ & $\operatorname{ls}\left(0^{\circ}\right.$ & $0^{\circ}$ & $180^{\circ}$ & $0^{\circ}$ & $180^{\circ}$ & $0^{\circ}$ \\
\hline & $90^{\circ}$ & $270^{\circ}$ & $! 0^{\circ}$ & $270^{\circ}$ & $\varphi()^{\circ}$ & 是元 & $270^{\circ}$ & $90^{\circ}$ & $270^{\circ}$ & $90^{\circ}$ & $270^{\circ}$ & $90^{\circ}$ \\
\hline & $180^{\circ}$ & $0^{\circ}$ & $180^{\circ}$ & ()$^{\circ}$ & $180^{\circ}$ & $0^{\circ}$ & $0^{\circ}$ & $180^{\circ}$ & $0^{\circ}$ & $180^{\circ}$ & $0^{\circ}$ & $180^{\circ}$ \\
\hline & $270^{\circ}$ & $90^{\circ}$ & $2 \pi 0^{\circ}$ & $9\left(0^{\circ}\right.$ & $270^{\circ}$ & $90^{\circ}$ & $9\left(0^{\circ}\right.$ & $270^{\circ}$ & $90^{\circ}$ & $270^{\circ}$ & $90^{\circ}$ & $270^{\circ}$ \\
\hline
\end{tabular}

Table 5.1: Phase cyeles for .MQMIAS/III:TC'OR 
The utilit.: of the MIQ.MISS/III:I'COR cxperiment is well-demonstrated in Figure 5.5 on $\mathrm{Na}_{3} \mathrm{P}_{3} \mathrm{O}_{9}$. Experimental conditions are chosen to closely mimick the regular HETCOR experiment. (Figure 5.3). For cxample, the sample is spun at $5 \mathrm{kHz}$ in both cases, and the contact time is sol to $5 \mathrm{msec}$ in both experiments. As expected, the ${ }^{23} \mathrm{Na}$ dimension contains two isotropic peaks (3ppm, FWHM) a.t -5.0 and $-24 \mathrm{ppm}$. These numbers are different. from those observed in DAS/HETCOR due to the different field strengths and scaling factors. Again, four distinct cross-peaks are observed, confirming the conclusion of a fully coupled ${ }^{23} \mathrm{Na} /{ }^{31} \mathrm{P}$ spin network. Analogous to the fact that DAS/HI:T(!)R has many advanlages over DAS, MQMAS/HETCOR has similar advantages orer DAS/IIETC'OR. For example, the most important one is that technically, MQM $\triangle S / I I E^{\prime} I^{\prime}(!) R$ is much simpler because dynamic-angle spinning probe is not a prerepuisite. T'o perform ('P a.t $0^{\circ}$, a static-coil DAS probe is required, which is nol a railablo rurremly from most. NMIR probe venders. As a comparison, our MQMAS/IIETC:OR experiment was done on a doubly-tuned MAS probe, which is available in most modern solid-stal.e NMR labs.

The technical simplification of MQMAS' also gives another advantage that makes

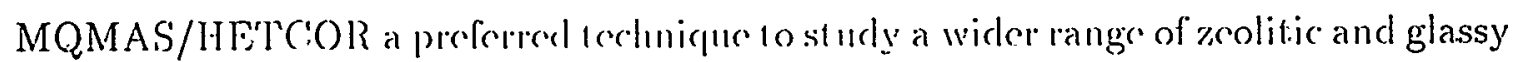
materials that contain muclei with short spin-lat.lice rolaxation limes (for instance, ${ }^{27} \mathrm{Al}$ and ${ }^{11} \mathrm{~B}$ ). As an example, ('hmolka ch al. showed thate regular "H/27 Al HETCOR experiment is ablo to discriminate acidic silcs in zcolite callalysts. Incorporating MQMAS to this exporimemt is natumal and would colhance the ${ }^{27} \mathrm{Al}$ dimension resolution by an oreler of magnitude. Anowher (xample shomn by Amoureux recently

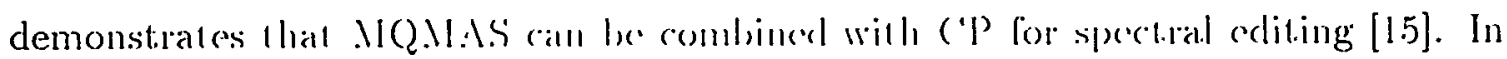

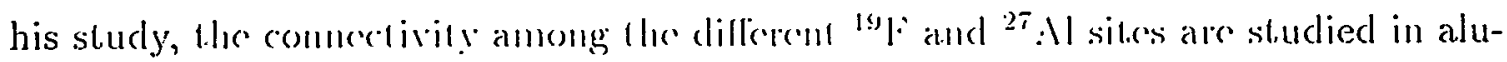
minum phosphate \%olites. MLQMAS/HIETCOOR in this case may lead to a direct mapping of the connertivity topology: More importantly. MLQMAS/HETCOR with 


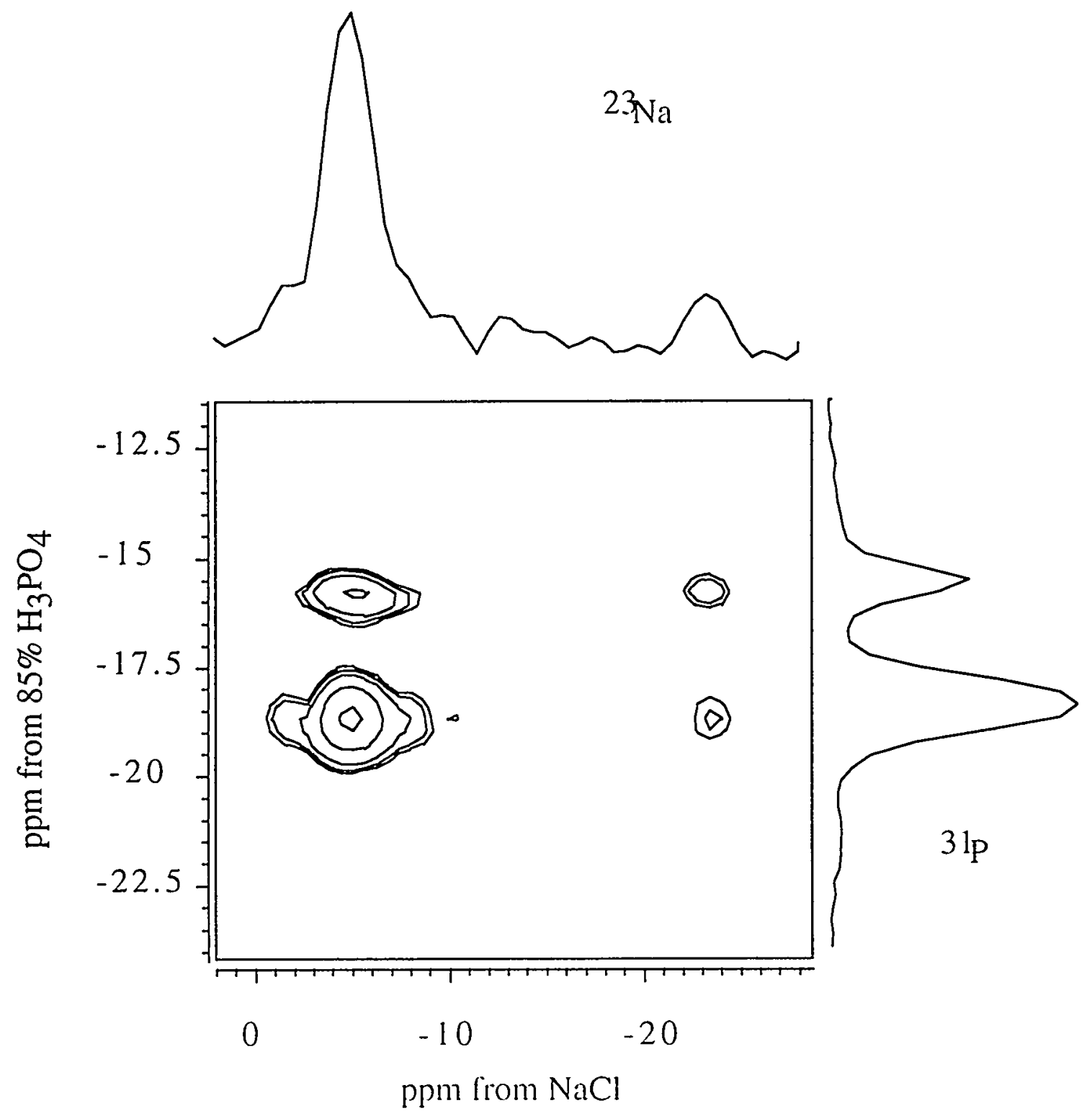

Figure 5.5: Two-dimensional ${ }^{23} \mathrm{Na}^{31} \mathrm{P}$ MQMAS/HETCOR spectrum of $\mathrm{Na}_{3} \mathrm{P}_{3} \mathrm{O}_{9}$

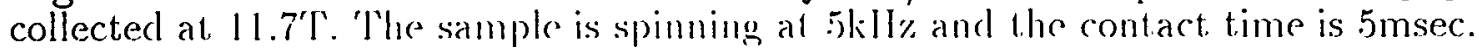


${ }^{27} \mathrm{Al} /{ }^{29} \mathrm{Si}$ spin pair can polentially be used to study a whole spectrum of minerals and zeolites. In all of these cases. DAS/HETCOR would fail due to short $T_{1}$ for ${ }^{27} \mathrm{Al}$.

Another potential advantage of MQMAS/HETCOR experiment for spin- $\frac{3}{2}$ is that the resolution in the quadrupolar dimension may be better than that observed in the DAS/HETCOR experiment for two reasons: (1), the DAS linewidth for ${ }^{23} \mathrm{Na}$ is determined by homonuclear dipolar interaction, which is not averaged out efficiently in DAS and often gives larger linewidth than MQMAS. For example, the ${ }^{23} \mathrm{Na}$ linewidth in DAS/HFTCOR is aboul, $800 \mathrm{~Hz}$ (FWHM), whereas that in the MQMAS/HETCOR spectrum is only $400 \mathrm{H} \%$ (2), the scaling factors of the chemical and quadrupolar shifts in MQMAS is larger than 1 (or smaller than-1). This better resolution is crucial, for example, in diflerentialing sodium sits in sodium phosphate and silicate glasses [1:3:3].

The disadvantage of MQMAS/IIPTC'OR is again associated with the inefficiency of excitation and reconversion pulses. Compared with the DAS/HETCOR spectrum (which is not quantitative cither). the intensity of the low frequency ${ }^{23} \mathrm{Na}$ site is much lower than the site population expected from XRD. Fven though simulations show that low frequency site with larger cluadrupolar compling constant has one-quarter of intensity of the other sile due to different excitation and reconversion efficiencies, it is not clear how much of the discrepancy can be attributed to this effect. Crosspolarization, as discussed carlier. introduces cxt ra problems that are hard to quantify. Because of this, MQMAS/HI:T'OR and D):S/LI:TC'OR experiments should both be considered as qualitalive. malter han quantilative.

It is worth noting that different mulliple-quantum excitalion and reconversion schemes can be combined with IIPT'(O) to bether quantify the MQMAS/HETCOR spectra. While this partially solves the cuantification problem, it is very likely that the problem will still exist. Fven lhough there is a scheme that claims quantitat- 
ive MQMAS excitalion and recomersion [0:3]. Hhe walidity of it is still limited to high excitalion power and small varialions in lhe quadrupolar coupling constants for different sites.

\subsection{Conclusion}

In summary, high-resolution IIVTCOR spectra involving quadrupolar nuclei can be obtained by correlating DAS or MQMAS spertrum of the quadrupolar nucleus with the MAS spectrum of the spin- $\frac{1}{2}$. 'The two resultant techniques are complementary to each other that provide qualitative chatacterization of the spin coupling networks. These techniques woukd have dircet application to sodium phosphate glasses, where the characteri\%ation of sitc distribulion and connectivity network is essential to the understanding of local orrloring in amorphons materials. With the new resolution and spectral editing apabilitics. detailed insperdion of local microstructure in various classes of technologically important matcrials is possible. 


\section{Chapter 6}

\section{Switching-Angle Spinning of Quadrupoles}

One of the major goals in the other chapters of this thesis is to determine the isotropic chemical shift $\left(\delta_{i s o}\right)$ and the quadrupolar coupling parameters $\left(C_{Q}\right.$ and $\left.\eta_{Q}\right)$. The anisotropic chemical shift interaction that a quadrupolar nucleus also experiences is only briefly discussed. The chemical shift anisotropy, however, like the quadrupolar interaction, contains valuable information about: the local geometry around the nucleus and is the major structural probe for spin- $\frac{1}{2}$ nuclei. This chapter is concerned primarily with the extraction of the full chemical shift, parameters and the relative orientation between the (S.S t.ensor and the quadrupole tensor.

\subsection{Overview}

Study of coexistent intoraction tensors by NMR can be dated back to the early sixties [134, 135]. There are al Icast. wo major reasons that such a study is crucial. First, the NMR spectra can nol be well-understood or reproduced by theoretical calculations if only one of the interactions is assumed to be dominating; second, in the case of coexisting dipolar and chrmical shift tensors, the dipolar tensor is often coaxial with the intermolecular vector. Lnowing the relative orientation between the chemical shift and the dipolar tonsors allows a direct mapping of the chemical shift tensor to the molecular frame [136i]. In Hhe case of coexisting chemical shift and quadrupolar tensors, mapp)ing the quadrupolar I cusor frame onto the chemical shift tensor frame may lead to a bertice pict ure of the rela lionship between the quadrupolar interaction and the molecular structure.

The most accurate medhod for the study of coexisting chemical shift and quad- 
rupolar tensors is the single-crystal medlod [1:37]. By carefully reorienting the single crystal sample in the static magnelic ficld, the change of the NMR peaks is recorded and analyzed. Good agroment between experimental data and fittings can often be achieved. The method, whilc being accurate, is tedious and sometimes impossible since the growth of the single-crystal may be nontrivial.

Simulating the static NMR spectra is anolher approach that was used by Bray et al. in 1969 [13.4] and by Cheng ot al. in 1990 [85] for the study of ${ }^{51} \mathrm{~V}$ and ${ }^{87}$ R.b, respectively. 'The simulation inrolves a.t least. $\&$ parameters, and multiple-field or multiple-nuclear experiments are often required; thus, the technique is not very powerful, especially in casces where multiple sites exist in the sample. For example,

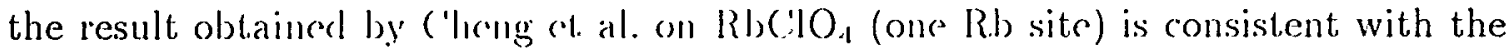

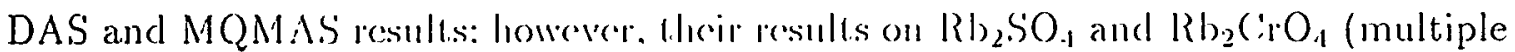
sites) are much less accurate and arc inconsistent with the data from high-resolution techniques [2-1].

Simulating the M:AS spectra [138, 139)] has the similar problem as simulating the static spectra. 'The adrantage is, howerer, that some small interactions (for instance,

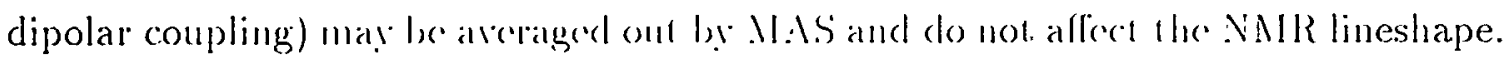
This may be a disadvantage as well. since chemical shilt inleraction is also averaged and its effect on the sperela is only refleceled by the sideband innensity.

The above lwo tecluniques, while boing less accumate, are quite simple and straightforward. Another interesting approach is 10 simmlate he whole MAS spectra, including the satellite lanusitions [1 II) I II]. This lype of spectm often has enough information 10 const rain lde simulation. and dereminalion of rery small chemical shift anisotropies has bern reporterl. The experiment may, howerer. be diflicult and requires well-calibratod hardware. Since the spect ra may cover up to a. $1 \mathrm{MIl}$ frequency range, a single experiment does not sulfice to excite the whole spectral range. In this 
case, multiple spectra aro recorded with different carrier frequencies. The resultant spectra have to be scaled and combined carefully to give the final spectrum.

The major problem with the previous simulation method is that the spectra are not sensitive enough to small or intcrmediale chemical shift interaction. A natural extension is then to exploil. a second dimension. This idea has been applied to the coexisting dipolar and chemical shift lensors, where a separated local field(SLF) NMR technique maps out; both interactions and their relative orientation to each other in two separate frequency domains [S]. I would like to demonstrate in this chapter that it is equally possible to extract cuadrupolar and chemical shift tensor orientation through a two-dimensional switching-angle spinning (S.AS) experiment.

\subsection{Theory}

\subsubsection{Coexisting Tensors}

In this section, only the coexistonce of chemical shift and quadrupolar tensors is considered, even though the hasic lheory is applicable to of her coexisting tensors. As discussed in chapter two, Hor quadrupolar and chemical shift interactions are characterized by wo and thres paramelors respectively. These parameters are $C_{Q}$ and $\eta_{Q}$ for quadrupolar intraction and $\delta_{i s o} \delta_{C S S}$ and $\eta_{C s}$ for CS $\Lambda$. These parameters are defined in the principal axis framc (P:SS) of each intoraction and the two frames

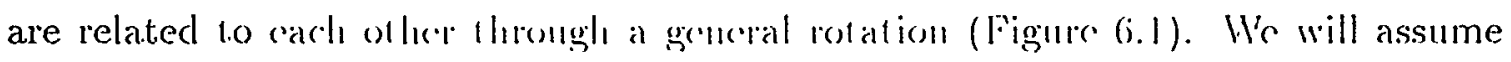

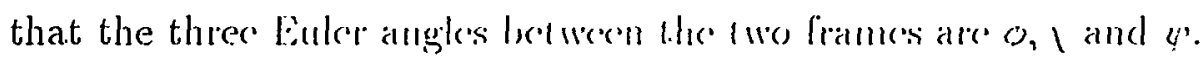

Calculating the powder NAIR spectra under spinning condition involves an ensemble average over all the crystallil.s. Ilo can specily each crystallite by giving the three Euler angles $\left(0^{Q} \ldots 3^{Q} . \gamma^{Q}\right)$ betwern line quadrupolar PAS and the rotor frame. The frequency shift for this crostallite has contributions from the chemical shift in- 


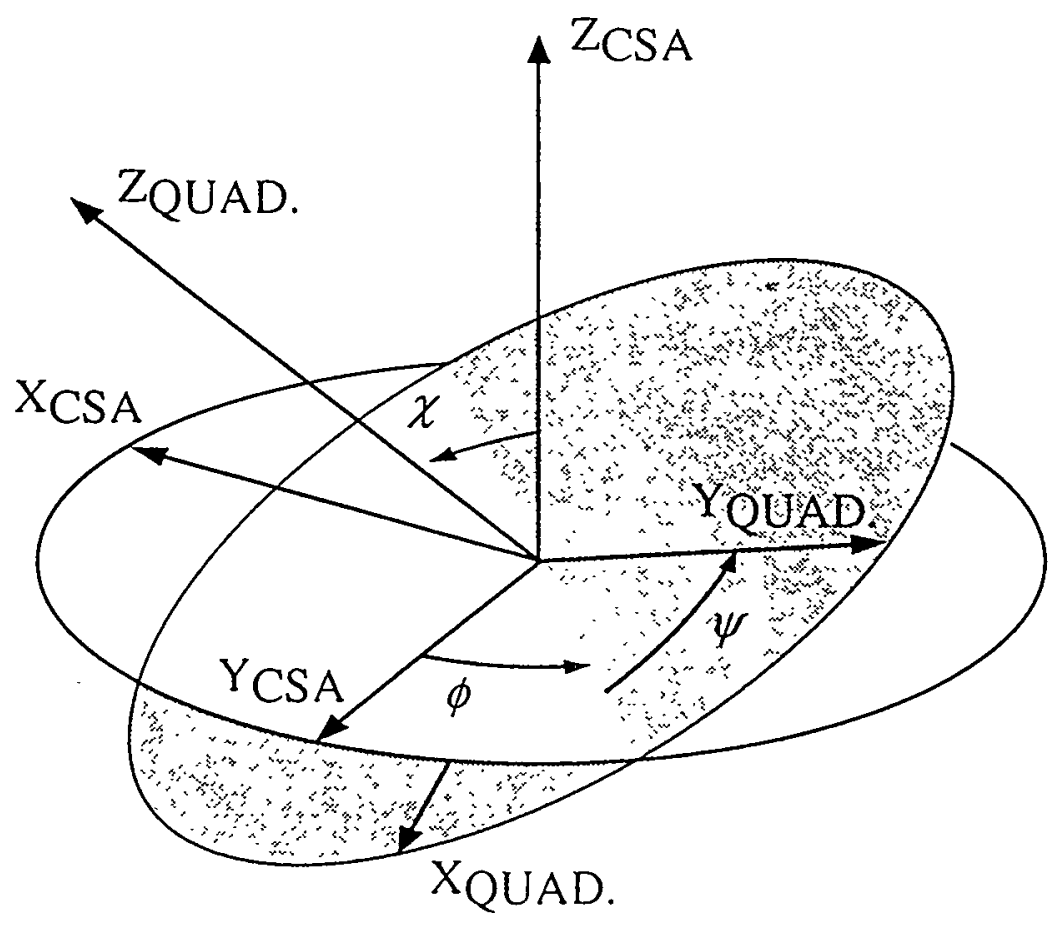

Figure 6.1: A schematic representation of the quadrupolar and chemical shift principle axis systems (PAS) with a relative orientation characterized by the Euler angles $\phi, \chi$ and $\psi$.

teraction and the quadrupolar intoraction.

$$
\Omega=\omega^{\prime r}+w^{2 Q}
$$

Eqn 2.60 and Eqn 2.66 can bre uscd to calculate the relevant terms in the above equation. For the quadrupolar shift, the quadrupolar tensor is first transformed into the rotor frame, and hien to the laboratory frame. This is exactly the same as what we did in chaphor 2 and the final result is tign 2.77. For the chemical shift interaction, however, an ext ra rotation from its PXS 10 llo quadrupolar PAS is needed.

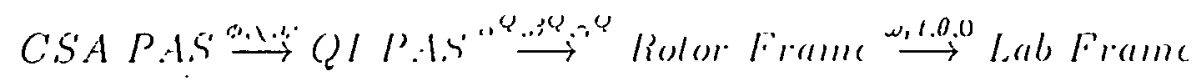

Eqn 2.68 needs to be modified to include this rotation.

$$
A_{20}^{C S}=\sum_{k=-2}^{2} D_{k+1}^{(2)}\left(\omega_{r} / .0 .0\right) \sum_{l=-2}^{2} \sum_{l=-2}^{2} D_{n l}^{(2)}(0,3, \gamma) D_{l k}^{(2)}\left(\phi^{Q}, l^{Q}, \psi^{Q}\right) \rho_{2 n}^{C S} .
$$


Exact expansion of this equation is tedious and complicated, but the final chemical shift would have the following format.

$$
\omega^{C S S}=\omega_{i s o}+A_{2}^{C S}\left(\alpha, \beta, \gamma, \phi^{Q}, \chi^{Q}\right) P_{2}(\cos \theta)
$$

\subsubsection{Switching-Angle Spinning}

The experimental scheme of switching-angle spinning (SAS) $[142,143]$ is shown in Figure 6.2, together with the pulses applicd. The experiment is in fact, similar to a DAS experimenl for quadrupoles (Figure 3.7). The difference is, however, that the two angles $\left(\theta_{1}, \theta_{2}\right)$ are not onc of the DAS angle pairs. Most often, the experiment is used to comelate the isolropic and anisotropic chemical shift spectra of a spin- $\frac{1}{2}$ nucleus. For this reason. one of the spinning angles is chosen to be the magic-angle to give high-resolution in one dimension. In our experiment, we also keep the magicangle as one of the angles, since MAS narrow's the quadrupolar lineshape by an order of magnitude. Different, from the spin-half case, both dimensions of an SAS spectrum of a quadrupolar nucleus do not have high-resolution.

This two-dimensional SAS exporiment can bo viewed as mapping the resonance frequencies of a powder sample onto a lwo-dimensional frequency plane, whereas a 1D experiment, maps those frequencies onto a onc-dimensional axis. Since the sample is spinning at two different, angles cluring the two lime previods, the resonance frequencies in the two dimensions for a single crystallito are diflerent. "Lhe two-dimensional map then reflects the correlation botwern llese frecpuencies. More specifically, the intensity of the resulting SAS spectrum is proportional to the probability lhat a nuclear spin having NMR freculuency al $\theta_{1}$ and we al. $)_{2}$.

For a powder sample the NMR frequency as a funclion of orientation is not single valued. Hence, for onc-dimensional spectra there is overlap of signals corresponding to crystallites with different. orientalions. Milun llo NMR frecpuencies are sampled 


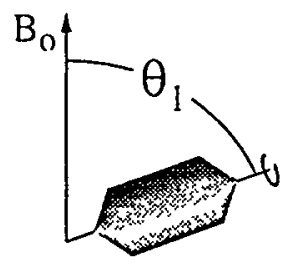

$\pi / 2 \quad \pi / 2$

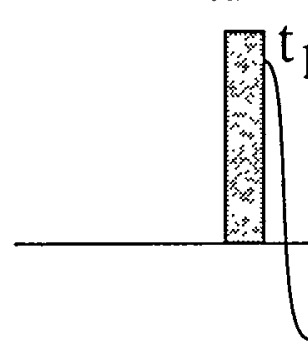

hop

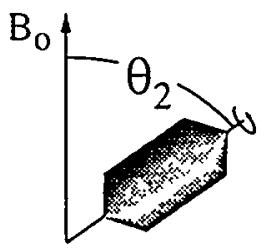

$\pi / 2$

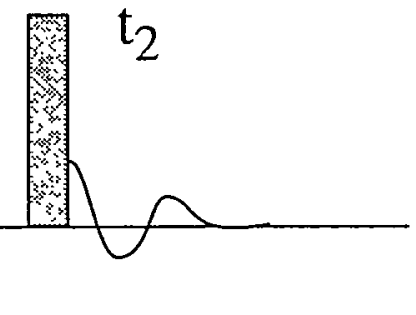

Figure 6.2: A schemalic representation of lihe switching-angle spinning (SAS) experiment. As the sample is spinning about. $D_{1}$ relative to the magnetic field, a $90^{\circ}$ RF-pulse is applied. The magnetiralion evolves in the plane transverse to the magnetic field for a time $l_{1}$ until another $R F^{2}$-pulse stores the magnetization along the field axis. The spinning axis is then changed to $0_{2}$. A final RF-pulse places the magnetization back into l.he transverse plane where it is detected. The experiment is repeated with $l_{1}$ incremonted by a dwoll time.

for two times in an SAS exporiment. undor different spinning conditions, the relative contributions of chemical shift and quadrupolar interaction are different for the two dimensions. The resulting sportla thus hare less overlapping and botler reflect the relative orientation betwern the lwo tensors.

The two-dimensiomal S: 1.5 specta can be simulated by calculating the following integration over all crystallites.

$$
I\left(\omega_{1}, \omega_{2}\right)=\int_{0}^{2 \pi} \int_{0}^{\pi} \delta\left(\omega_{1}, \Omega_{1}\left(\alpha^{Q}, . \beta^{Q}\right)\right) \delta\left(\omega_{1}, \Omega_{1}\left(\alpha^{Q}, \beta^{Q}\right)\right) \sin \beta^{Q} d \alpha^{Q} d \beta^{Q}
$$

Here, $\Omega_{1}$ and $\Omega_{2}$ are the resonance frecpuencies in liefn 6.1 , and I)iac $\delta$-lunction is used. To see how the SAS spect a depend on ho quadrupolar palameters, ligure 6.3 shows the calculated spectra with difforont spiuning angles and quadrupolar asymmetric parameters $\left(\eta_{Q}\right)$. For the simulation. $0_{2}$ lats berol set to the magic-angle, and only

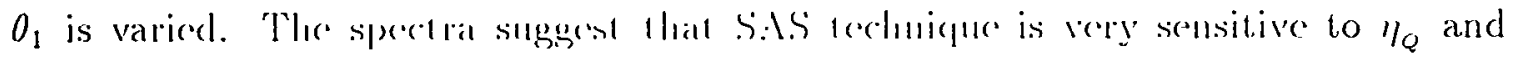


produces well-defined t.wo-dimensional lineshapes.

\subsection{Experiment}

All samples used in the experiment were obtained from commercial sources, typically with a stated purity of $99.8 \%$. The ${ }^{23} \mathrm{Na}$ and ${ }^{87} \mathrm{Rb}$ NMR spectra were acquired at $4.2 \mathrm{~T}\left({ }^{23} \mathrm{Na}, 49.1 \mathrm{MHz} ;{ }^{87} \mathrm{Rb} 60.8 \mathrm{MHz}\right), 9.4 \mathrm{~T}\left({ }^{23} \mathrm{Na}, 105.9 \mathrm{MHz} ;{ }^{87} \mathrm{Rb} 130.9 \mathrm{MHz}\right)$ or 11.7T $\left({ }^{23} \mathrm{Na}, 132.3 \mathrm{MHz:}{ }^{87} \mathrm{Rb}\right.$ 163.6 MHz), with a Nalorac Quest, Bruker AM-400 or a Chemagnetics CMX-500 spectrometer, respectively. A home-built NMR probe based on the design of Eastman et al. [ $7 \cdot 2]$, capable of fast reorientation of the spinning axis, and employing a Doty S'cicnlific (Columbia, SC) $5 \mathrm{~mm}$ fast MAS stator was used [73]; except for the ${ }^{23} \mathrm{Na}$. NMR spectra acquired at 4.2T, using a static-coil DAS probe based on the design of Mueller et al. [23]. A Whedco (Ann Arbor, MI) high torque stepping motor and motor controller were used to reorient the rotor axis in typically $40 \mathrm{~ms}$. The spinning axis was initially set to $54.74^{\circ}$ using the ${ }^{81} \mathrm{Br} \mathrm{NMR}$ signal of $\mathrm{KBr}$. The pulse sequence we used are similar to the DAS sequence and same phase cycle was used. To ensure selective excilation of the central transition, $90^{\circ} \mathrm{RF}$ pulses were typically longer than $10 /$ s. Ciencrally, 128 and 512 points were acquired in $t_{1}$ and $t_{2}$, respectively. with 32 scans por $l_{1}$ value. However, the experimental parameters depended groally: on sample and fickd strength. During processing, the $t_{1}$ dimension was \%cro fillod to 250 points and $100 \mathrm{H} \%$ Gaussian line-broadening was applied in both dimensions. Dilute acuncous solutions of $\mathrm{RbNO}_{3}$ and $\mathrm{NaCl}$ were used as external standards. ('nlike DAS or MQMAS, no shear transformation is needed in this case. The simulations were performed on a Silicon Ciraphics (Mountain View, CA) R4000 workstalion using a program written in FORTRAN. A two-dimensional spectrum with 128 points in both dimonsions takes approximately three seconds to calculate. 


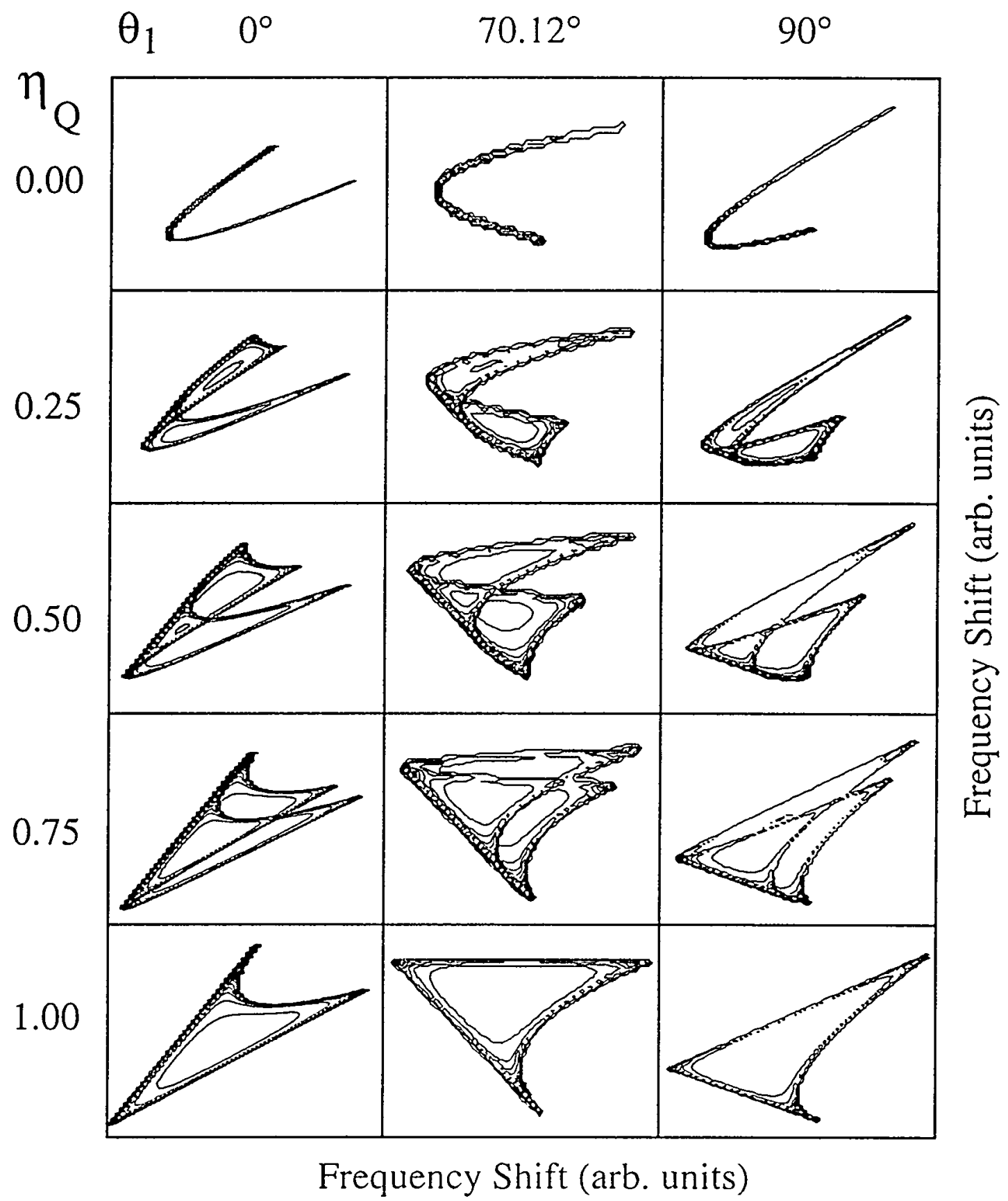

Figure 6.3: Simulated two-dimusional SAS spectra, considering only the quadrupolar interaction, as a function of $)_{1}$ and llac (puadrupolar asymmetry parameter, $\eta_{Q}$. $\mathrm{O}_{2}$ is $54.74^{\circ}$. 'The horizont al dimension is 1 he MAS dimension. 


\subsection{Results}

As a test of the technique and the simulation program, a sample was chosen that had been studied previously and contains one crystallographically distinct sodium site that has negligible chemical shift anisotropy [41]. In Figure 6.4, the experimental and simulated ${ }^{23} \mathrm{Na}$ SAS NMR spectra of $\mathrm{Na}_{2} \mathrm{SO}_{4}$ acquired with $\theta_{1}$ and $\theta_{2}$ equal to $80^{\circ}$ and $54.74^{\circ}$, respectively, are shown. The magic-angle was chosen to minimize the CSA

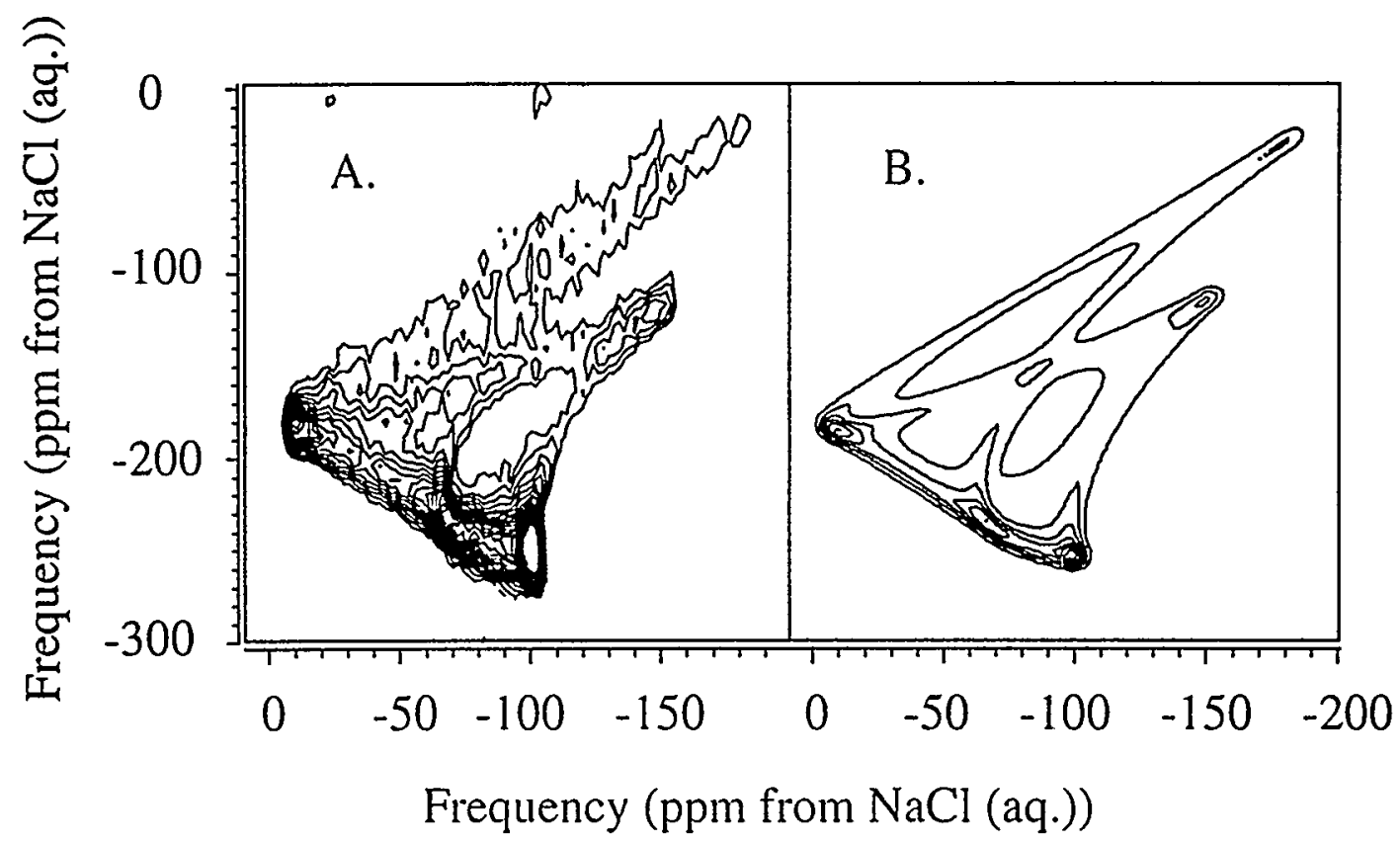

Figure 6.4: (A) Experimental and (B) simulated two-dimensional ${ }^{23} \mathrm{Na}$ SA.S NMR spectra of $\mathrm{Na}_{2} \mathrm{SO}_{1}$ acquired at $4.2 \mathrm{~T}$ with $0_{1}=80^{\circ}, O_{2}=54.74^{\circ}$. The simulated spectrum corresponds to $C_{Q}=2.6 \mathrm{MHz}, \eta_{Q}=0.6$, and $\delta_{i s o}=4 \mathrm{ppm}$. No chemical shift anisotropy was included in the simulation. The horizontal dimension is the MAS dimension.

effect in this dimension: the other angle was chosen to be near to $90^{\circ}$ to minimize the sideband intensity. The simulated spect.rum yields $C_{Q}=2.6 \mathrm{MHz}, \eta_{Q}=0.6$, and $\delta_{i s o}=4 \mathrm{ppm}$. consistent, with previously reported values. The projection of the $\omega_{2}$ dimension corresponds to an MAS spect.rum and is consistent with an MAS spectrum acquired separately. as well as with a simulation of the one-dimensional 
spectrum calculated using the same parameters as above. For simple systems, the quadrupolar parameters are obtainable using the SAS technique, with an accuracy equal to or greater than that obtained from one-dimensional magic-angle spinning spectra.

Many two-dimensional spect ra were calculated to determine the effects of a small anisotropic chemical shift. $\left(\delta_{C S}\right)$ on SAS spectra. For $I=\frac{3}{2}$ and a moderate $C_{Q}(3 \mathrm{MHz})$ at a resonance frequency of $100 \mathrm{MHz}$, a $\delta_{c s}$ of less than $10 \mathrm{ppm}$ is difficult to detect. However, a $\delta_{C S}$ of $15 \mathrm{ppm}$ can canse discernible changes in the spectral features. The effect of the chemical shift anisotropy on the two-dimensional lineshape depends on the relative orientation of the lwo principlo axis systems and is more significant when the two principle axis systems are not coinciclent, especially when $V_{Z Z}$ and $\delta_{Z Z}$ are not parallel.

Shown in Figure (6.5) is a ${ }^{37} \mathrm{RL}$ S.SS NMIR spertrum of $\mathrm{Rb}_{2} \mathrm{SO}_{1}$ acquired at $9.4 \mathrm{~T}$; also shown separately are the spectira of the individual sites and the corresponding simulations. $\mathrm{Rb}_{2} \mathrm{SO}_{4}$ has tho rubidium sites that are resolved with $0_{1}$ ecpal to $90^{\circ}$. The spectrum is howerer. not resoled at the magic-angle. Thus the correlation of spectra at two angles by SAS coukl lad to the determination of quadrupolar parameters for both siles.

In a separated MAS spertrum acepuired with l.he sample spinning at $10 \mathrm{kHz}$, spinning sidebands are still apparent due to the large quadrupolar interactions and result in the intensity of ho lwo siles orerlapping in the one-dimensional MAS spectrum (not shown here). In the S:LS spectrum the signal is spread into a two-dimensional frequency planc yiclding groatcr resolution and noarly complete separation of the two sites. The simulations of the S.1S spectrum yield $C_{Q}{ }_{Q}=5.3$ and $2.6 \mathrm{MHz}, \eta_{Q}=0.1$ and $1.0, \delta_{t s o}=10$ and $10 p p m$ for lhe lwo sites. respertively. These results are consistent with those obtained by Balt isberger from fick-dependent. I)AS measurements 


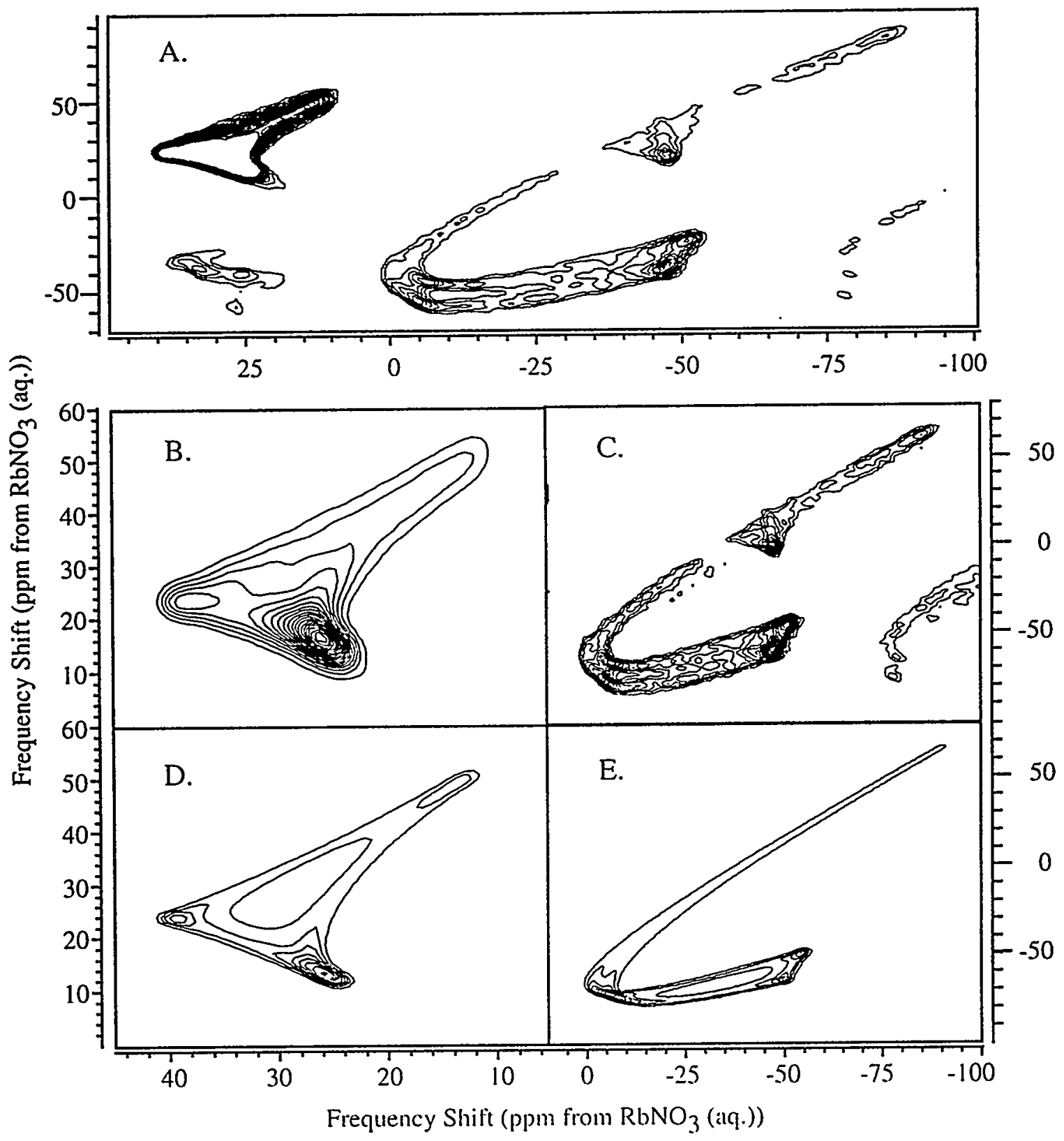

Figure 6.5: Experimental and simulat.cd two-climensional ${ }^{87}$ Rb SAS NMR spectra of $\mathrm{R}_{2} \mathrm{SO}_{4}$ a.cquired a.t $9.4^{\mathrm{T}} \mathrm{T}$ with $\mathrm{O}_{1}=90^{\circ}, 0_{2}=54.74^{\circ}$. The simulated spectra were calculated for $C_{Q}=5.3$ and $2.6 \mathrm{MII} \%, \eta_{Q}=0.1$ and 1.0 , and $\delta_{i s}=16$ and $40 \mathrm{ppm}$, respectively. The hori\%ontal dimension is the MAS dimension. (A). SAS spectrum with 2-sites (Contour levels: 1\%-20\%, 1\% increments). (B) and (D): Experimental and simulated spectra for site I with $C_{Q}=2.6 \mathrm{MH}$ (Contour level: $5 \%-100 \%, 5 \%$ increments). (C) and (E): Experimental (Contour levels: $0.5 \%-10 \%, 0.5 \%$ increments) and simulated (Contour levels: $5 \%-100 \%, 5 \%$ increments) spectra for site II with $C_{Q}=5.3 \mathrm{MHz}$. 
[24], by Fernandez from simulating MASs spect.ra [139], and for the $C_{Q}=2.6 \mathrm{MHz}$ site by Cheng et al. [85] from measurement.s of static powder samples. Although, Cheng et a]. [85] reported much different values for the $C_{Q}=5.3 \mathrm{MHz}$ site. Overlap between the two sites in the one-dimensional MAS spectrum complicates the accurate determination of these parameters using one-dimensional techniques.

The discrepancy between the simulated and experimental spectra shown in Figure 6.5 may be due to the anisotropic chemical shifts of both sites. The anisotropic chemical shifts have been reported by Fernandez et al. [139] from simulations of one-dimensional MAS spectra to be 12 and $35 \mathrm{ppm}$ for the $C_{Q}=2.6$ and $5.3 \mathrm{MHz}$ sites, respectively. The relative orientation of the principle axis systems was also reported, though with large uncertainties. While in some cases a better agreement between the experimental and simulated SAS spectra is obtained by including anisotropic chemical shifts, simulations incorporating their results do not match the experimental spectrum better than simulations neglecting the anisotropic chemical shift. This opens the question of how accurate the chemical shift parameters can be determined by one-dinensional NMR when the chemical shift anisotropy is relatively small. Further refinements of the two-dimensional SAS spectra are needed to accurately determine the small anisotropic chomical shifts and the relative orientation of the principle axis systems.

In Figure 6.6, the ${ }^{8 \pi} \mathrm{Rb} S A S$ NMR spectrum and projections of $\mathrm{Rb}_{2} \mathrm{CrO}_{4}$ acquired at $11.7^{\prime} \mathrm{T}$ with $0_{1}$ and $0_{2}$ (cplial to $70.12^{\circ}$ and $54.74^{\circ}$. respectively, are shown. One-dimensional MAS spectrum of llis sample was shown in chapter 3 and the magnitude of CSA was estimated there to be around 110ppm ( $\left.\delta_{C S S}=-110 \mathrm{ppm}\right)$. The two-dimensional SAS lineshape results from the combination of quadrupolar and chemical shift interactions. The projection above the contour plot is indistinguishable from a M $M S$ spectrum of tho central-transition measured independently. Both 


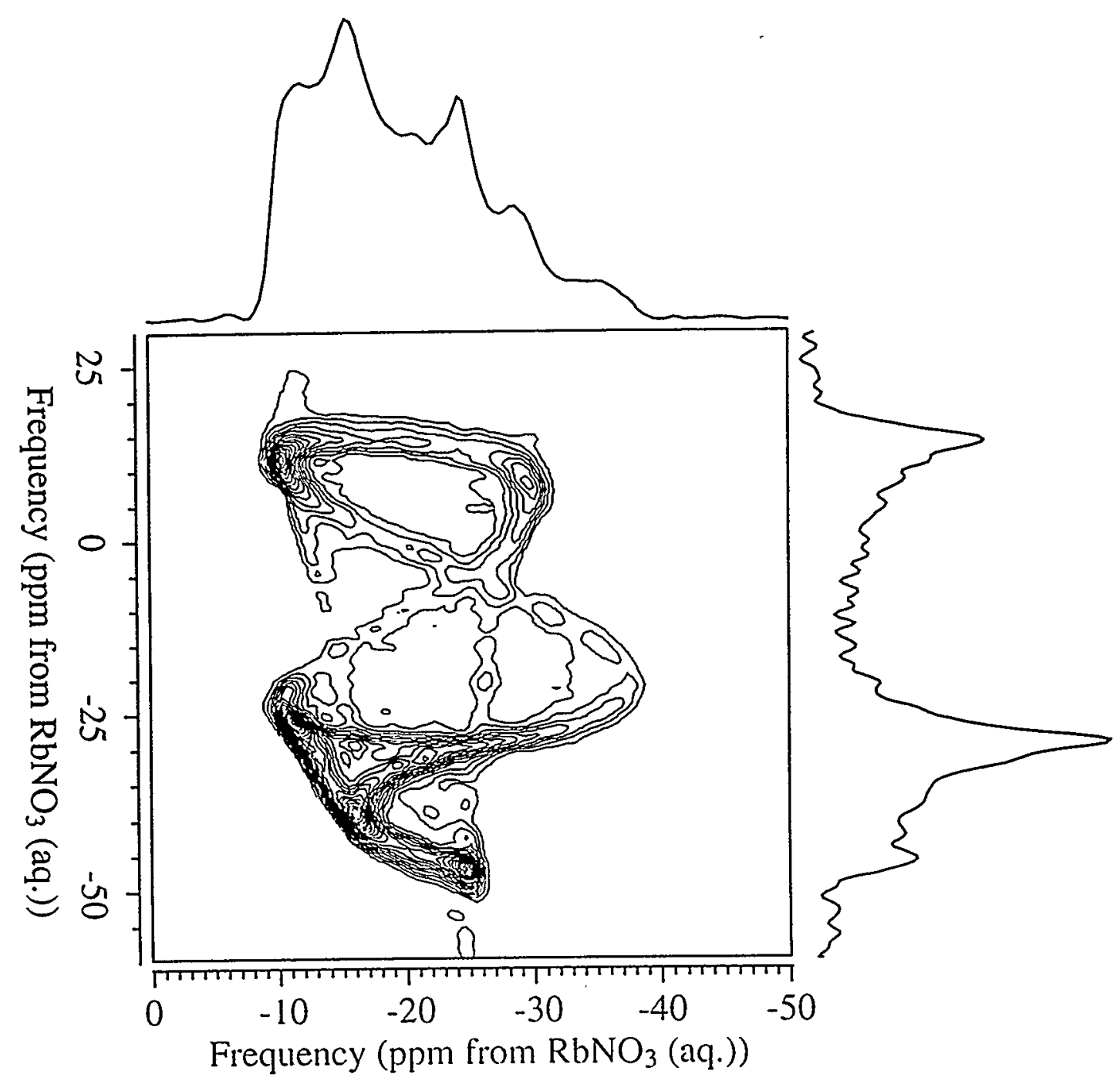

Figure 6.6: Experimental two-dimensional ${ }^{8 i}$ R.b SAS NMIR spectrum and projections of $\mathrm{Rb}_{2} \mathrm{CrO}_{2}$ acquired at. $11.7 \mathrm{~T}$ will $0_{1}=70.12^{\circ}, \mathrm{O}_{2}=54.74^{\circ}$. The horizontal dimension is the MAS dimension. The projections in both dimensions are also shown. 
one-dimensional projections have structure: however the two-dimensional lineshape contains more detail. While $\mathrm{Rb}_{2} \mathrm{CrO}_{4}$ has two rubidium sites, the site with the smaller quadrupolar interaction is selectively obscrved. The MAS spectrum of $\mathrm{Rb}_{2} \mathrm{CrO}_{4}$ at $11.7 \mathrm{~T}$ has more structure than if only the quadrupolar interaction was present. Both the centerband and spinning sidebands are affected, since the spinning speed is not fast enough to completely average the anisotropic chemical shift. The MAS spectra of $\mathrm{Rb}_{2} \mathrm{CrO}_{-1}$ at various fields were simulated using the parameters determined from the SAS experiments, and reproduce most of the features in the centerband and sidebands of the experimental spectia.

In Figure 6.7, the ${ }^{8 i}$ Rb S SS NMR experimental and simulated spectra of $\mathrm{Rb}_{2} \mathrm{CrO}_{4}$, obtained with $\theta_{1}=70.12^{\circ}$ and $\theta_{2}=54.71^{\circ}$ accuired a.t $4.2,9.4$ and $11.7 \mathrm{~T}$ are shown. The differences among the three mosured spectra reflect the dependence of the chemical shift and quadrupolar intcractions on magnetic ficld strength. The smaller the magnetic field, the smaller the chemical shift intcraction is relative to the quadrupolar interaction. Note that the spectrum acquired at the lowest field, 4.2T, appears similar to spectra in Figure 6.3 calculated considering only the quadrupolar interaction. All the simulated spectra in Pigure 6.7 were calculated using the following parameters, $C_{Q}=3.5 \mathrm{MHz}, \eta_{Q}=0.3, \delta_{i s i}=-\tau \mathrm{ppm}, \delta_{C S}=-110 \mathrm{ppm}, \eta_{c s}=0, \lambda=70^{\circ}, \psi=0^{\circ}$. $\phi$ is undefined because. in this case the chemical shift interaction is axially symmetric $\left(\eta_{c s}=0\right)$. The fact that the same parameters fil the spectira acquired at three field strengths rigorously demonstrates the precision of the technigue. "The quadrupolar coupling constant, quarlrupolar asymunct parameder and isotropic chemical shift are all consistent with the walues deformined using fick-dependent DAS' measurements by Baltisberger ol al. [2 1$]$.

To determine the sensitivity of the spect ra to the chemical shift parameters, spectra were calculated with $\delta_{c}, s$ and $\eta_{r . s}$ aried separatoly by $\pm 1.5 \mathrm{ppm}$ and from 0 to 


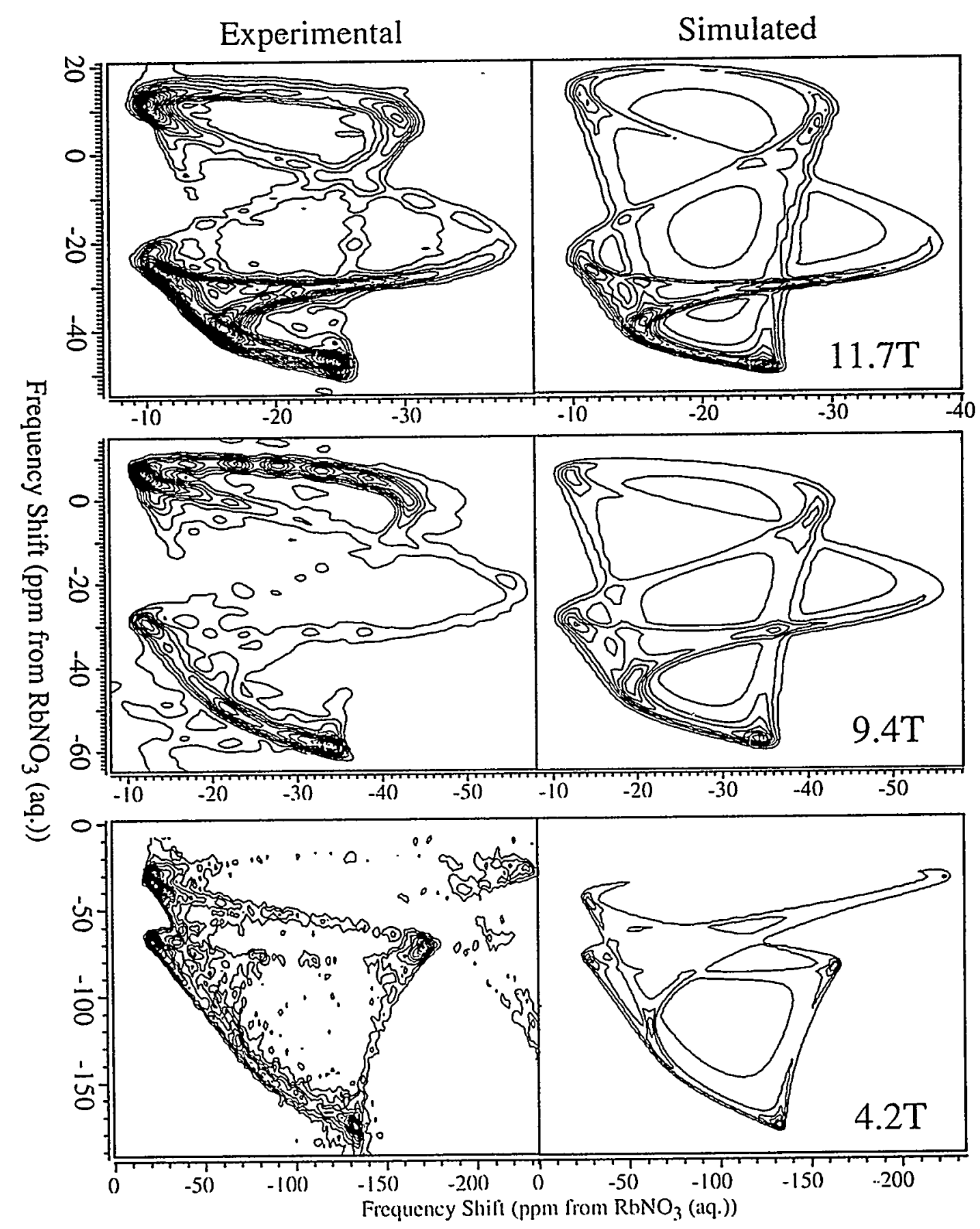

Figure 6.7: Experiment.al and simulated two-dimensional ${ }^{87}$ Rb SAS NMR spectra of $\mathrm{Rb}_{2} \mathrm{CrO}_{4}$ measured at $4.2 \mathrm{~T}, 9.4 \mathrm{~T}$, and $11.7 \mathrm{~T}$ with $0_{1}=70.12^{\circ}, \theta_{2}=54.74^{\circ}$. The same parameters were used for the simmlated spectra at all three fields strengths, and are $C_{Q}=3.5 \mathrm{MHz}, \eta_{Q}=0.3, \delta_{\text {iso }}=-7 \mathrm{ppm}, \delta_{c s}=-110 \mathrm{ppm}, \eta_{c s}=0, x=70^{\circ}, \psi=0^{\circ}$. The horizontal dimension is the MAS dimension. 
0.15 , respectively, with all the romaining parameters iclentical to those used for the simulations shown in Figure 6.7. From simulated spectra such as those presented in Figure 6.8 , the uncertainty in $\delta_{C S S}$ and $\eta_{C S}$ is determined to be $\pm 15 p p m$ and less than 0.15 , respectively. To determine the sensitivity of the simulated spectra on $\chi, \psi$ and $\eta_{Q}$ the spectra presented in Figure 6.9 were calculated with parameters identical to those in Figure 6.7 except that $1, y^{\prime}$ and $\eta_{Q}$ were varied separately by $\pm 5^{\circ}$, from 0 to $15^{\circ}$, and \pm 0.1 respectively. The simulated spectra calculated with $\psi$ equal to $+15^{\circ}$ or $-15^{\circ}$ are equivalent. From simulations such as those shown in Figure 6.9, conservative error estimates for $\backslash, \psi^{\prime}$ and $\eta_{0}$ arr $\pm 5^{\circ} . \pm 15^{\circ}$. and \pm 0.1 , respectively. Considering the greater accuracy in cletermining of compared to 7 , and similar results reported by Fernandez et al. [1:30]. onc might suspect that this is a general trend. Further experiments on other systems can be performed to clarify this point.

Thus, the chemical shift and quadrupolar interaction parameters and the relative orientation between the principle axis systems are determined with the following accuracy: $C_{Q}=3.5 \pm 0.2 \mathrm{MH} \mathrm{L}, \eta_{Q}=0.3 \pm 0.1, \delta_{\mathrm{sso}}=-7 \mathrm{ppm}, \delta_{C s}=-110 \pm 15 \mathrm{ppm}$ $\eta_{c s}=0 \pm 0.15, \mathrm{r}=70^{\circ} \pm 55^{\circ} . u^{\prime \prime}=0^{\circ} \pm 15^{\circ}$. with $\phi$ undefined. These results differ significantly from those determined from onc-dimensional NMR spectra of powder samples reported by ('heng et al. [S.5].

\subsection{Conclusion}

NMR has the potential to chatactorize the local alomic enviromment in materials and can be used to dofomine structure properly redationships, location and distribution of substitution species. and motion and diffusion of aloms, as well as other technologically important propertics in inorganic solids. With NMR, an experimentalist has the unprecedented advantage of being able 10 manipulate the Hamiltonian of the system under study using molio frecpucney pulses and sample spinning, among 


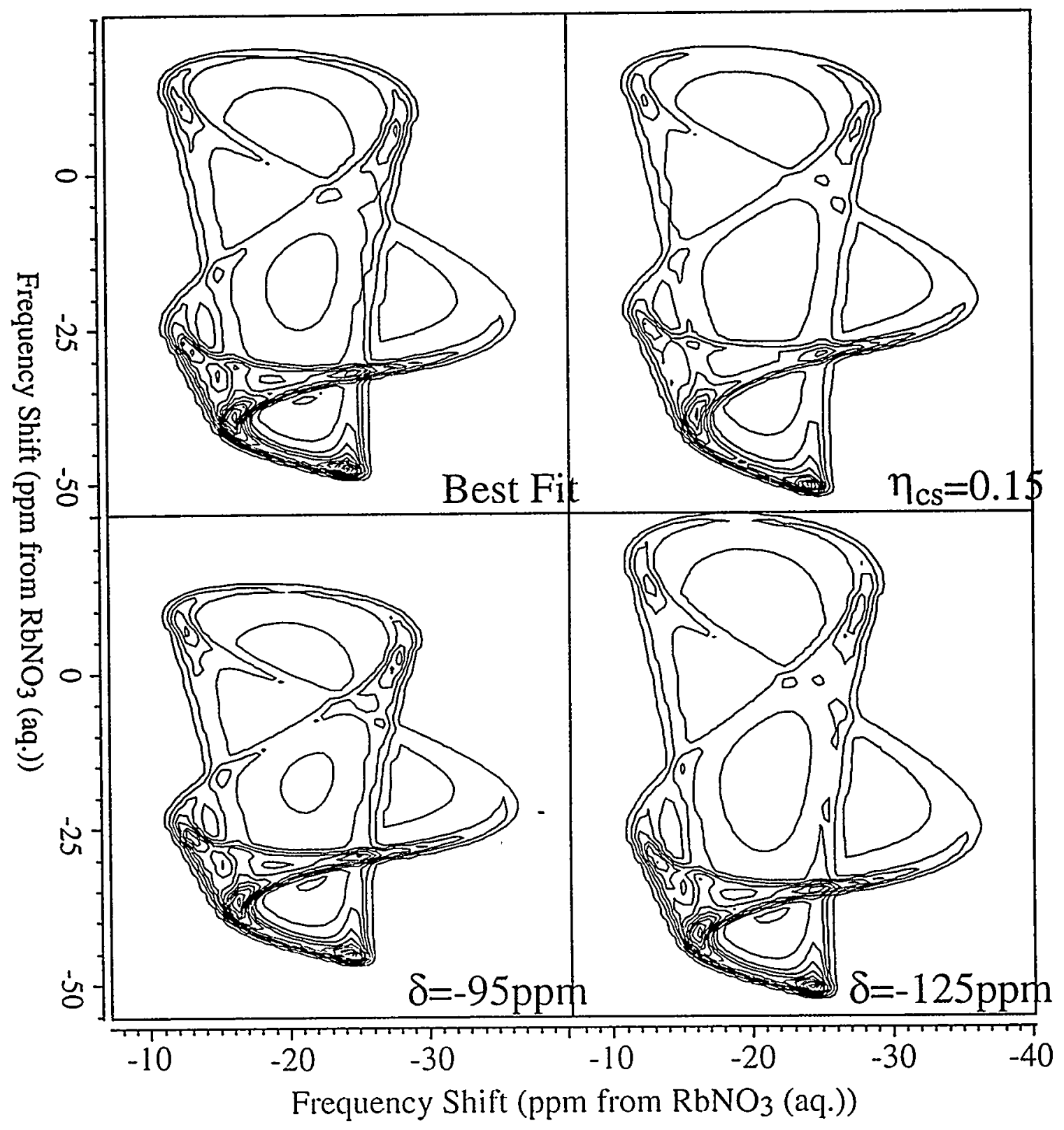

Figure 6.8: Simulated two-dimensional ${ }^{5 \pi} \mathrm{R} . \mathrm{b}$ SAS NMR spectra of $\mathrm{Rb}_{2} \mathrm{CrO}_{4}$ at $11.7 \mathrm{~T}$ with $O_{1}=70.12^{\circ}, 0_{2}=54.74^{\circ}$. The same parameters $\left(C_{Q}=3.5 \mathrm{MHz}\right.$, $\eta_{0}=0.3, \delta_{i s o}=-7 \mathrm{ppm} . \delta_{C s}=-110 \mathrm{ppm}, \eta_{c s}=0, \lambda=70^{\circ}, \psi=0^{\circ}$ ) were used for all the simulated spectra except where noted. The horizontal dimension is the MAS dimension. 


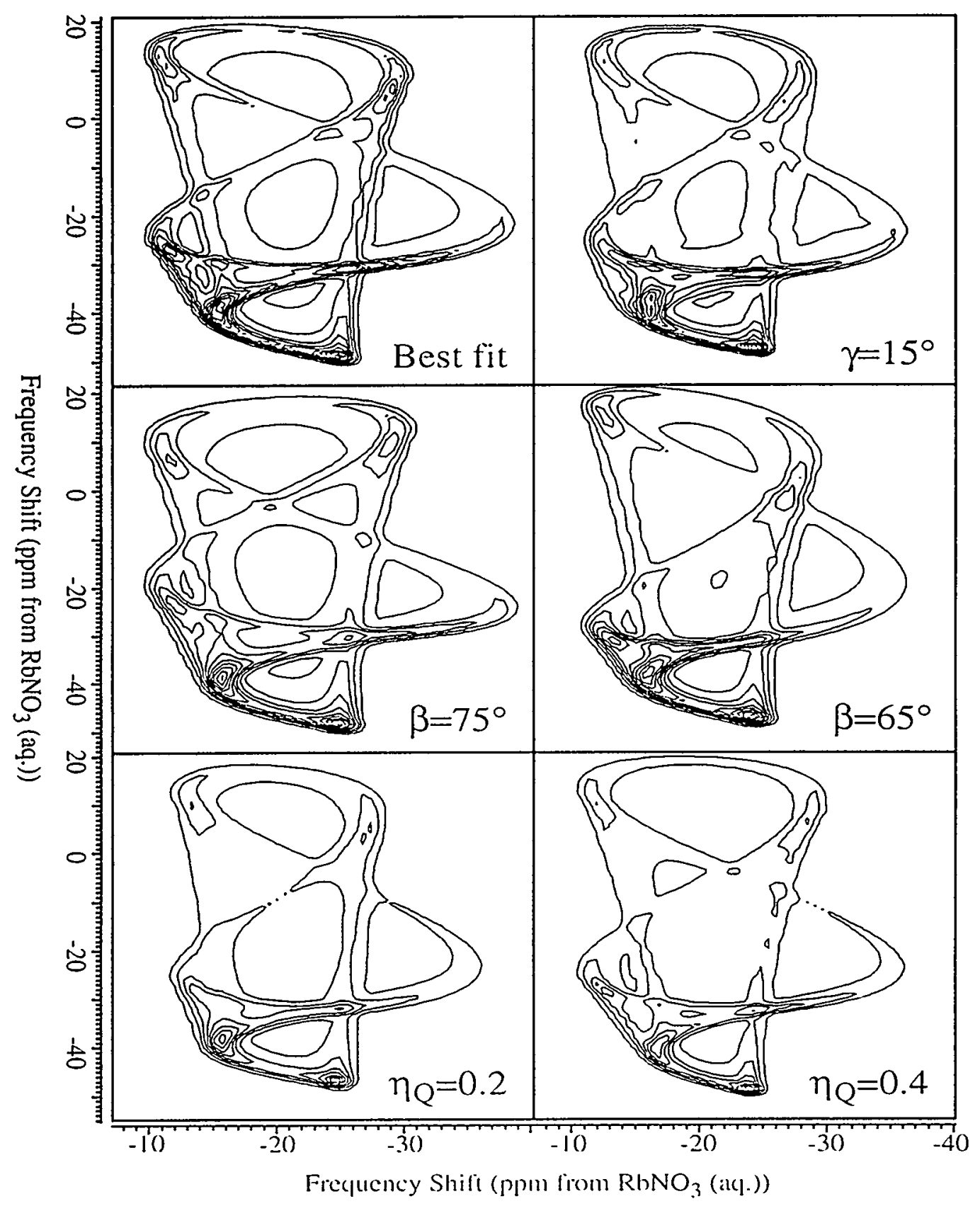

Figure 6.9: Simulated two-dimensional"iRl, SSAS NMIR spectlat of $\mathrm{Rb}_{2} \mathrm{CrO}_{4}$ at $11.7 \mathrm{~T}$ with $\left.\theta_{1}=70.12^{\circ}, 0\right)_{2}=5.1 .71^{\circ}$. The same paranclers $\left(C_{Q}^{\prime}=3.5 \mathrm{MHz}\right.$, $\left.\left.\eta_{Q}=0.3, \delta_{\text {iso }}=-7 p \mid \mu m, \delta_{(s)}=-110 \mathrm{p}\right) \mathrm{m}, \eta_{c s}=0 . \beta=70^{\circ}, \gamma=0^{\circ}\right)$ wcre used for all the simulated spectra cxept where noted. 'The horizontal dimension is the MAS dimension. 
other techniques. The combination of these methods with multi-dimensional NMR techniques enables the direct correlation and/or deconvolution of multiple interactions. These ideas have been used in this chapter to correlate the quadrupolar and chemical shift interactions and to determine the quadrupolar and chemical shift tensor elements, as well as the relative orientation between the two principal axis systems for a rubidium site in $\mathrm{R}_{2} \mathrm{CrO}_{4}$. Extension of these methods to a three-dimensional experiment by incorporating a purely isotropic dimension will be applicable to systems with multiple atomic sites and those with a distribution of jsotropic shifts such as glasses. In this case, variable-angle correlation spectroscopy can be incorporated with SAS to simplify the technical requirement of the experiment $[86,144]$. 


\section{Appendix A}

\section{A Short Review on MQMAS}

This appendix gives a review on MQMAS', which after being proposed by Frydman [3], received considerable and extensive attention in the past years. Our contribution at Berkeley, as described in the preceding cliapters, represents a very small fraction of the problems and applications come with this technique. While much of the work from other groups has been montioned before, a review here makes this thesis a more complete reference.

\section{A.1 History}

Multiple-c|uantum NMR on half-integer quadrupoles has been studied for more than two decades. Most experiment.s wre performed on single-crystals and the results served as a nice demonstralion of creation and detection of multiple-quantum coherences in the solicl-state $[6.1,65.115,1.16,147]$. The difficulties that hinder the application to powder samples is a gencral bolief that the excitation and reconversion of the multiple-culantum coherences are inefficiont for powder samples.

A moureux was probal)ly tho first to explicitly derive the second-order quadrupolar Hamiltonian associat.ed to symmet.ric multiplc-quantum transitions ( $m \leftrightarrow-m)$ for powder samples under spinning condition [20]. The results showed that the dependence of this IIamiltonian on the spinner axis is different to that of the central transition only by some constant factors. 'The magnified chemical shift differences were the primary driving force of his study. The possibility of using multiple-quantum coherences to enhance the resolulion of a fluadrupolar NMR spect.rum was not realized until Frydman [3] proposed the MQMAS experinemt two years later. 
Research interests on MQMAS then fall into two groups, aiming at technique development and application respectively. The questions that the first group of people are interested in include the efficient acquisition of MQMAS spectra and extending MQMAS to inclucle other features for better spectral interpretation; the latter group concerns more about systematic studies of interesting materials in the solid-state.

\section{A.2 Technique Development}

Three types of questions are among the primary goals of the first group. The first one involves data acquisition, processing and spectral interpretation. Due to the similarities between DAS and MQMAS, much of the DAS variations were directly applied to MQMAS, making this cliscipline somewhat mature now. The second problem is about the excitation of multiple-quantum coherence and its reconversion to observalble single-quantum coherence. This turns out as a difficult problem that remains unsolved. The third direction is toward the utilization of MQMAS principles to other experiments for spectral editing and characterization.

\section{A.2.1 Data Acquisition}

The first MQMAS spectrum presented by Frydman [3] is a 1D spectrum. Acquisition of pure-alosorplion phase l.wo-dimensional MQMAS spectra was the topic of a subsequent: paper [51] and also the topic of many other publications $[48,49]$. In principle, this problem is the same as the accuisition problem with DAS and all of the DAS solutions $[-16,50]$ are equally applicable here. For example, the $z$-filter technique used by Mueller [16] to give pure-absorption phase DAS spectra was used by Amoureux [48] and Wimperis [19] to acquire pure-absorption MQMAS spectra. It is worth noting that this solution, which was shown not optimal for the DAS case [50], is not optimal for MQNAS cither. A simple redefinition of the isotropic dimension 
with possible use of whole-echo acquisition for D $\Lambda S$ is superior to the $z$-filter method. Even though it was claimed that z-filcer gives slightly less spectral distortion, the 2-4 fold sacrifice in sensitivity may act as the killing factor for an MQMAS experiment.

The whole-echo method, which gives better sensitivity and has been primarily applied in DAS for ${ }^{23} \mathrm{Na},{ }^{87} \mathrm{Rb}$ and ${ }^{17} \mathrm{O}$, may not be a good choice for nuclei such as ${ }^{27} \mathrm{Al},{ }^{11} \mathrm{~B}$ even though they' sometimes show long spin-spin relaxation times. The possible problem with this improvement is that the spin-spin relaxation time for different sites might be different. and whole-echo acquisition makes the spectra less quantitative. The decision on whether or not this modification should be used must be left to the experimenter.

Mossiot pointed out that data accuisition with rotor synchronization yields spectra without sidcbands and gives better M $\Lambda \mathrm{S}$ dimnsion lineshapes [148]. The acquisition method is also more sensitive, due to the smaller spectral width one needs to cover in the high-resolution dimcnsion. Ilis result shows that when the spectra have a lot of sidcbands, unsy'nchronized accuisition may give centerband pattern quite different from perfoce quadrupolar lineshape and lead to errors in estimating the quadrupolar parameters. 'The downside of the experiment is that fast spinning ( $>10 \mathrm{kHz}$ ) is a priori since the spectral widh is now coupled with the spinning speed and a slow spinning spered means a small spectral window that may not cover all the different sites.

Recently there have bern many pajers about the avoidance of shearing transformation $[49,55]$. 'Thusse papers do not make significant improvement on the old acquisition scheme. THe modified experiment still requires intensive manipulation of the time-domain data thal most NMR softwares do not provide directly. 


\section{A.2.2 Spectral Interpretation}

Without high-resolution techniques such as DAS, DOR and MQMAS, $\delta_{i s o}, C_{Q}$ and $\eta_{Q}$ are determined primarily by simulating the quadrupolar lineshape. With DAS and DOR, lineshape simulation is avoidable if experiments are performed at more than one field strengths. Since $C_{Q}$ and $\eta_{Q}$ are often coupled together, only the quadrupolar product $P_{Q}$ can be derived and two experiments with different fields suffice to the determination of $P_{Q}$ and $\delta_{i s o}$. Even though, lineshape simulation is still preferred since it gives better quantification, and it derives $C_{Q}$ and $\eta_{Q}$ separately.

Interpretation of MQMAS spectra requires a bit more effort since the chemical and second-order quadrupolar shifts are scaled differently other than DAS and DOR. Multiple-field experiments are not necessary but. preferred. Different spectral interpretation methods have been shown in Chapter 4 . For more complicated cases that the observed DAS slift is not, available, multiple-field experiment or spectral simulation is still the right choices.

As described in detail in chapter 3, MQMAS usually gives narrower lines than DAS due to the removal of homonuclear dipolar couplings. The broadening due to heteronuclear dipolar coupling can however be overcome by decoupling. The scaling of the observed frequency in MQMAS spectra gives MQMAS better or worse resolution (compared to DAS). depending on the spin cuant.um numbers and the transitions observed.

MQMAS and DAS sidcband patterns are very similar [45, 83]. The sidebands are not integer multiples of the spinning rate allay from the centerband. This is clue to the scaling factors introduced in the shearing procedure. 


\section{A.2.3 Excitation and Reconversion Pulses}

The conventional way' of exciting triple-quantum coherence is to use a pair of $90^{\circ}$ pulses, separated by a shorl poriod of lime inversely proportional to the magnitude of the quadrupolar interaction. The conversion from the triple-quantum coherence back to single-quantum cohcrence is achieved by a single $90^{\circ}$ pulse. This scheme was not efficient to excite siles experiencing large quadrupolar interactions [3]. Most of the MQMAS applications use other excitation methods.

Using single long pulse for excitation of t riple-cuantum coherence can be dated back to late seventies [(j), 146]. Amourenx first showed that a simple single strong pulse is equally effective for the creation of triple-cuantum coherence and reconversion of this coherence to single-cuantum colerence in a spinning sample [149]. The method was separately worked out by (iriffin at al. later [68]. Except for the spin-locking method proposed by (iriffin [6:3] for spin- $\frac{3}{2}$ nuclei, this simple excitation scheme seems to be the most cfficient onc, and is used widely now. How to choose the lengths of the pulses to achieve best clficiency was the topic of many subsequent publications $[58,67]$.

There scem to bo somc inconsistencies in litcrature on how to choose the excitation and reconversion pulse durations. For cxample, Amoureux [67] suggests that excitation pulses of $210^{\circ}, 180^{\circ}, 120^{\circ}$ and $90^{\circ}$ should be used for $I=3 / 2,5 / 2,7 / 2$ and 9/2 nuclei, respectively: (iriffin of al. [68'] showed, however, that a $540^{\circ}$ pulse is efficient for $\mathrm{I}=3 / 2$ nuclei: our (xporiments also suggest.s that $540^{\circ}$ pulse is elficient. The discrepancy comes mainly from the different experimental conditions and conventions that different, rescarch groups uscel. In the simmlations performed by Amoureux, relatively high RP powor is assumod (100-200k H\%), whoreas most of our experiments were performed with a much lower power level (30-60kHz). When the power level is low, longer pulse is neoded to gain boller excitation efficiency (See Figure 3.14 
to 3.16). In addition, some factors (field strength, frequency offset, second-order quadrupolar interaction and (he spinning speed) that affect the excitation efficiency are not well-studied. While each of these factors may not greatly change the result, their combined effect may be significant and needs further study. Another factor that contributes to the seemingly controversial results is the different conventions used by different groups. Notice that, the pulse lengths reported by Amoureux are liquid-state pulse lengths, which are different. from solid-state pulse lengths by a constant factor dependent on the spin quant.um number. If converted into solid-state pulse lengths, the numbers they reported should read as $480^{\circ}$ for $I=3 / 2,540^{\circ}$ for $I=5 / 2,480^{\circ}$ for $I=7 / 2$ and $450^{\circ}$ for $I=9 / 2$. These rsults are not very different from the solid-state pulse lengths reported by us and Giriffin.

The conversion of the triplc-quantum coherence back to single-cuantum coherence is less effective than the excilation process $[58,67]$. A single solid-state $180^{\circ}$ pulse is often the most approprialie pulse length.

An interesting question with MQMAS is that even though the excitation and reconversion are not very officient, the MQ-filtered MAS dimension still resembles the quadrupolar lineshape under MIAS. This surprising result was explained by Frydman [58] using a very simple model. The cflect is attributed to sample spinning, which renders the excitalion much less orientation-dependent. A more rigorous treatment through simulation is still nol axalable. Such a treatment is important to the full understanding of the cxcitation process. ('urrently. most simulations treat the spin ensemble as a whole and report only the cuscmble averaged results. The results, while applicable in reality, lack physical intuition. It may be insighttful to classify spins with different orientations into many groups and see how each group of spins are affected by different cxcitalion schemes. The results may be more intuitive and may lead to better excitation mothods. 
An altemative excitation scheme that mili\%es spin-locking pulses was proposed by Griffin et al. [6:3]. According to their reports, the excitation method gives better quantification than the single-pulse method. The tenet of the method is that even for crystallites with large quadrupolar coupling constants, under MAS and spinlocking condition, the sfrective quadrupolar coupling constants go through 2 or 4 zero-crossings [69, 70]. 'The singlo-quantum coherence is transfered into multiplequantum coherence during spin-locking period. While the results shown in the paper are promising, it is not clcal why a better quantification is necessarily achievable, since the spin-locking cllicicucy for half-inleger nuclei is orientation-dependent and inefficient too. Answer to this (puestion also requires a careful look at the response of each spin to RF pulses.

There are at least f wo of here groups that work on the application of shaped pulses for excitation. However. Ilecir results are not pessitive compared to the simple single pulse excitation and reconversion mochanisms. It is also worth noting that when the excitation fiedd strenglf cerecels some limit. further increase in $B_{1}$ does not necessarily lead to improved excitation chiciency: 'This conclusion is not woll tested, since in most NMR laboratories, very high RF power (>200kll\%) is still not available.

\section{A.2.4 Extensions}

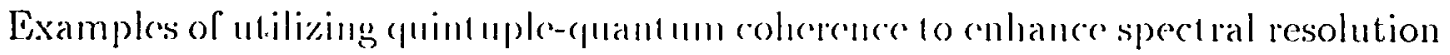

for spin- $\frac{5}{2}$ nuclei Was first domonstratcel by Amonroux [79]. 5QMAS gives better resolution than 3Q2M.AS. Iut has primarily locoll applied to ${ }^{27} \mathrm{Al}$ in zoolites with small quadrupolar compling constants [15. 79, 150]. Excilation of quintuple-quantum coherence is often difficult, thus this sperelal cnhancement mothod is only applicable to siles with vory small (quadrupolar compling const.ants (2-3) MHz).

An interesting extension of MQ MI AS is to use it for the measurement of chemical 
shift anisotropy (CSA) [-15]. For spin- $\frac{3}{2}$ nuclei, chemical shift effect is magnified by a factor of greater than 2 in the multiple-quantum isotropic dimension, and the second-order quadrupolar interaction is scaled down. CSA dominates the isotropic dimension sideband pattern when it is relatively large. Simulation of the isotropic dimension gives CSA parameters, even though the method does not provide detailed information about the relative orientation between the CSA and QI tensors. Another method that performs multiple-quantum experiment at $30.56^{\circ}$ or $70.12^{\circ}$ removes second-order quadrupolar interaction totally, and allows the direct determination of CSA parameters [-1.5]. The method however is limited to samples with moderate CSA of many kiloherl\%.

Combining MQMAS with (hoss-polarization (CP) was recently presented by Amoureux et, al. [15]. (IP was performed on ${ }^{19} \mathrm{~F} /{ }^{27} \mathrm{Al}$ pair and the resultant spectra suggested that fluorine is connected to only one type of the aluminum sites. The result is promising for spectral editing, but special care must be taken to interpret the CP/MQMAS spectra.

In the above $\mathrm{CP}$ exporiment. ${ }^{19} \mathrm{~F}$ single-quantum coherence was first transferred to ${ }^{27} \mathrm{Al}$ single-quantum coherence. which was then stored as $z$-magnetization. Triplequantum excitation was prrformed on this magnctizalion. The coherence transfer is somewhat inefficient since a z-filter is used. It is not clear if it is experimentally feasible to transfer the ${ }^{19} \mathrm{~F}$ single-cluantum coherence directly to ${ }^{2 \pi} \mathrm{A}$ triple-quantum coherence. Early experiments on ${ }^{2}$ II give positive signs even though ${ }^{2} \mathrm{H}$ usually has smaller quadrupolar coupling constant. [1-17].

In Chapter 5, we showed another (I) based experiment--MQMAS/HETCOR [151]. The experiment allows the registry of high-resolution HETCOR spectra for quadrupoles and maps out the spin network directly. The experiment was demonstrated on ${ }^{23} \mathrm{Na} /{ }^{31} \mathrm{P}$ pairs, but by uo means should be limited to those systems. 


\section{A.3 Application}

\section{A.3.1 $\quad{ }^{27} \mathrm{Al}$}

About half of the MQMAS applications by now involves aluminum $[60,61,79$, $150,152,153]$. The high abundance and high resonance frequency makes it very suitable for MQMAS studies. Also, aluminum is one of the most important nuclide in zeolites, mincrals, glasse's and other incresting materials. In zeolites, it was demonstrated that MQM:AS is able to differentiate aluminum sites with slightly different environmonts. 'The resolution is usually good enough compared to DOR, and could be further improved by exciting the quintuple-quantum coherences. In most of the zeolitic malorials studied so far, the quadrupolar coupling constants are small (2-5MHz). MQMIAS was also applied to the study of alumina calalysts. Once again, the quadrupolar compling constants are not loo large. A systematic study of aluminum in aluminosilicalc and aluminato mincrals has been presented in detail in chapter 4. The result. lhere suggest hlat. when ('o is large, the spectra are no longer quantitative. Two different. mothorls of rotricing quadrupolar parameters were also discussed there. It, was shown that. MQQMAS' is often able to differentiate aluminum sites with different. coordination mumbers. which is important in understanding the microstructure of glassy matrials.

\section{A.3.2 ${ }^{2 \cdot 3} \mathrm{Na}$}

Most of the ${ }^{23} \mathrm{Na}$ work wre performed on model compounds to demonstrate the efficiency of different experimental schemes. It was fomd that MQMAS is often effi-

cient for ${ }^{23} \mathrm{Na}$ as most siles hare small quadrupolar compling constants [58]. Sodium sites with small differences in chemical shifts and/or cuadrupolar coupling constants are differentiable from cach oflere will .MQMISS. 'The technique has been utilized 
to separate two strongly overlapping resonances from distinct sites in glasses and to detect impurities in $\mathrm{Na}_{2} \mathrm{IICO}_{3}[5 \mathrm{~T}]$.

\section{A.3.3 ${ }^{11} \mathrm{~B}$}

Even though ${ }^{11} \mathrm{~B}$ sites usually have small $C_{Q}$ values, published MQMAS results on this nuclide is surprisingly rare. In principle, ${ }^{11} \mathrm{~B}$ spectra are easy to obtain. As a very positive example, Hwang et al. presented a series of ${ }^{11} \mathrm{~B}$ spectra of $\mathrm{B}_{2} \mathrm{O}_{3}$ and $\mathrm{B}_{2} \mathrm{~S}_{3}$ glasses that up to \& boron peaks were identified in a single spectrum [62]. The superior resolution offered by MQMAS would grcatly contribute to the understanding of borate or borosilicalc glasses.

\section{A.3.4 ${ }^{17} \mathrm{O}$}

Compared to the wide application of DAS to ${ }^{17} \mathrm{O}$, MQMAS of ${ }^{17} \mathrm{O}$ is somewhat limited. According to our calculation, 3QMAS and 5QMAS are both not adequate to resolve multiple-sites with varying Si-O-Si bond angles. The conclusion seems to

find more support in the past yoar [12T]. Our experiment on zeolite $Y$ yielded at the most two pealis whereas DAS gives 3 well-resolved peaks. For non-bridging oxygen, however, MQMAS is as powroful as DAS in resolving overlapping pealss.

It is clear that non-briclging and bridging oxygen sites usually gives different peaks in the isotropic dimcnsion [116, 126. 12 7$]$. Same conclusion is also true for oxygen in Si-O-Si and Si-O-. Il fragments [56]. Based on the high-resolulion available from MQMAS, kinetics of ${ }^{17}()$ labeling [116] has bern studied to show that Si-O-Si and Si-O-Al have diflerent. raclivit.y. Such an offerel was first observed in ZSM-5 zeolite through ${ }^{18} \mathrm{O}$ labeling [154]. and recently received more interests in other areas. 


\section{A.3.5 Less Common Quadrupolar Nuclides}

MQMAS on ${ }^{8 \pi} \mathrm{Rb}[45,51],{ }^{15} \mathrm{Sc}[45],{ }^{55} \mathrm{Mn}[3]$ were also performed. The results serves as demonstration of the potential applicability of MQMAS to a series of other nuclei. My experience with nuclides such as ${ }^{65} \mathrm{Ga}$ and ${ }^{93} \mathrm{Nb}$ is however, negative. The large quadrupolar coupling constants are the major problem with the experiment, even though homonulcear dipolar coupling may introduce extra complications. 


\section{Bibliography}

[1] K. T. Mueller, B. Sun, G. C. Chingas, J. W. Zwanziger, T. Terao, and A. Pines. Dynamic-Anglc Spinning of Quadrupolar Nuclei. J. Magn. Reson., 86:470, 1990.

[2] A. Llor and .J. Virlet. Towards IIigh-Resolution NMR of More Nuclei in Solids: Sample Spinning witl. Time-Dependent, Spinner Axis Angle. Chem Phys. Lett., 152:248, 1988 .

[3] L. Frydman and J. S. Harwood. Isolropic Spectra of Half-integer Quadrupolar Spins for Bidimensional Magic-Angle Spinning NMR. J. Am. Chem. Soc., 117:5367, 1995.

[4] A. Abragam. Principles of N'uclcar Magnetism. Clarendon Press, Oxford, England, 1961 .

[5] R. R. Ernst. G. Bodenhausen, and A. Wokaun. Principles of Nuclear Magnetic Resonance in Onc and T'uro Dimınsions. Oxford University Press, New York, 1991.

[6] C. P. Slichter. Principles of Magnelic Resonance Springer-Verlag, Berlin, 1990.

[7] M. Mchring. Principles of ILigh Rrsolulion N.M/R in Solids. Springer-Verlag, Berlin, 1983.

[8] K. S. Rohr and II. W'. Spicss. Mlullidimensional Solid-Stale NMR and Polymers. Academic Press. London. 1994.

[9] G. Engelhardt. Migh-Resolulion Solid-S'tals X:MR of Silicales and Zeolites. John Wiley \& Sons. Now Lork. 1987.

[10] R. N. Zare. Angular Ifomonlum: l'nderslanding Sprotial Aspects in Chemistry and Physics. Joln Willey d: Sons. Vew York, 1988.

[11] J. J. Sakurai. Aderunced Quan/um .J/celuanics. Addison-Wesley Publishing Inc., Redwood (ily. California, 1985.

[12] C. C. Tannoudji, 13. Diu, and F. Laaloc. Quanlum Mechanics. John Wiley \& Sons, Paris, $197 \pi$. 
[13] A Pines, M. (i. Ciiblyy, and J. S. Waugh. Proton-Enhanced NMR of Dilute Spins in Solids. .. (\%.m. I/hy.s., 59):569) 1973.

[14] E.O. Stejskal, J. Schacfer, and J.S. Waugh. Magic-Angle Spinning and Polarization Transfer in Prolon-Enhanced NMR. J. Mrgn. Reson., 2S:105, 1977.

[15] M. Pruski, I). P. lang. ( . Fomando, and .J. P. Amoureux. Multiple-Quantum Magic-Angle Spinning N.MIR with ('ross-Polarization: Spectral Editing of HighResolution Spectia of Quadrupolar Nuclci. Solid Slale NMR, 7:327, 1997.

[16] J. E. Roberts, S. V'aga, and R. Ci. Ciriflin. Two-Dimensional Heteronuclear Chemical Shift. Correlation Spectroscopy in Rotating Solids. J. Am. Chem. Soc., 106:2506, 19\$1.

[17] A. Samoson. Satcllit.c Trausition Iligh-Resolution NMR of Quadrupolar Nuclei in Powders. (Hem Phys. Lr I1.. 1199:29, 1985.5.

[1S] G. Kóunath, P. Iosso, S'. Stcucrnagel. II. Schneider, and C. Jager. ${ }^{27} \mathrm{Al}$ satellite Transition Spectroscopy (sal ras) of Polyerystalline Aluminium Borate $9 \mathrm{Al}_{2} \mathrm{O}_{3} \cdot 2 \mathrm{~B}_{2} \mathrm{O}_{33}$. Solid Siale .VM/R, $1: 261$. 199.2.

[19] C. Jager. N'M/R Basie P'rinciples and Progress, vol. 32. page 1333. SpringerVerlag, Berlin IIridelluerg, I!9)1.

[20] J. P. Amonrenx. Iligh-Resolution Solicl-Sitate NMIR for Spin $3 / 2$ and $9 / 2$ - the

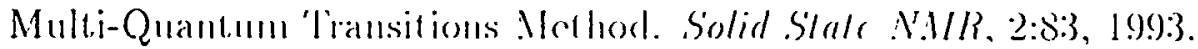

[21] M. M. Maricy and .J. S. Waugh. NMlR in Rotating Solids. J. Chem. Phys., $70: 3: 300,1979$.

[22] J. Horzack and :A.P. Borger. Sidchand Intonsities in NMIR Spectra of Samples

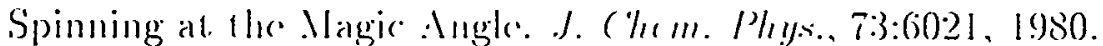

[23] K. T. Mucllar. Dymamir-.lugh Spimming and Double Rolalion of Quadrupolar Nuclei. Phl) Hesis. Iniversity of ('aliformia at Borkoley. 1991.

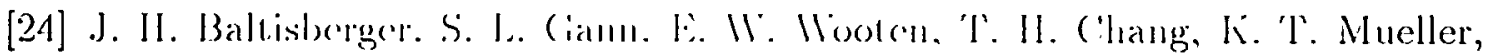

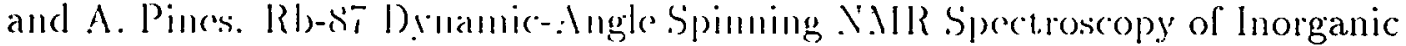
Rubidium Salıs. J. $1 \mathrm{~m}$. (\% m. Sor.. 111:T1s!). 19(9).

[25] F. Dyson. Time Orkering ()pcrator. Phys. Rer.. 75:168, 1919). 
[26] N. C. Nielsen, H. Bildsoe, and H. J. Jakobsen. Finite of Pulse Excitation in MAS NMR of Quadeupolar Nuclei. Quantitative Aspects and Multiple-Quantum excitation. Chem Physs. Lell., 191:205, 1992.

[27] N. C. Nielsen, H. Bildsoe, and H. J. Jakobsen. Multiple-Quantum MAS Nutation NMR. Spectroscopy' of Quadrupolar Nuclei. J. Magn. Reson., 97:149, 1992.

[28] B. Bloembergen, E. M. Purcell, and R. V. Pound. Phys. Rev., 73:679, 1948.

[29] J. Haase, K. D. Park. K. Guo, H. K. C. Timken, and E. Oldfield. Nuclear Magnetic Resonance Spectroscopic Studies of Spin-Lattice Relaxation of Quadrupolar Nuclei in \%colit.es. J. Phys. Ch.cm., 195:6996, 1991.

[30] J. Haase and E. Oldficld. Spin-echo Behavior in Nonintegral-Spin Quadrupolar Nuclei in Inorganic Solids. J. Magn. Reson. Serics A, 101:30, 1993.

[31] G. Bodenhausen, II. liogler, and R. R. Ernst. Selection of Coherence-Transfer Pathways in NMIR Pulse Lixporimonts. J. Magyn. Reson., 58:370, 1984.

[32] A. D. Bain. Coherence levels and coherence pathways in NMR. A Simple Way to Design Pliaso ('ycling Procedures. J. Mla.gn. Reson., 56:118, 1984.

[33] J. Keeler. Mullinuclear. Magnelie Resonance in Liquids and Solids-Chemical Applications, page 10:3. Kluwer Academic Publishers, Netherlands, 1990.

[34] D.J. States, R.A. Haberkorm, and D..J. Ruben. A Two-Dimensional Nuclear Overhouser Experiment wit.l, Pure Alssorption Phase in Four Quadrants. J. Magn. Reson., 48:286, 1982.

[35] E. R. Andrew, A. Bradlbury, and R. G. Bades. Nuclear Magnetic Resonance Spectra from a (rystal Rotalcel al Iligh speed. Nature, 182:1659, 1958.

[36] E. R. Andrew, A. Bradlbury. and R. (i. Backes. Removal of dipolar broadening of Nuclear Magnetic Resonance Spectia of Solids by Specimon Rotation. Nature, 18:3:1802, 19:5\%.

[37] S. Vega, E. T. Olcjuic\%ak, and R. (i. Ciriffin. Rotor frequency lines in the Nuclear Magnelic Rosonance Spect.ra of Rotaling Solids. J. C.hem. Phys., 80:4832, 1984 .

[3S] D. W. Aldcrman. M. S. Solum, and I). M. (irant. Methods for Analyzing Spectroscopic Lincshapes. X.IIR Solid Powder Pattorns. J. ('llcm. Phys., 84:3717, 
1986.

[39] Z. Zheng, Z. Gan. N.K. Sothi, D.W. Alderman, and D.M. Giant. An Efficient Simulation of V'ariablo-Angle Spinning lineshapes for the Quadrupolar Nuclei with Half-integer Spin. J. Magn. Reson., 95:509, 1991.

[40] S. Ganapathy, S. Shore and E. Oldfield. Variable-Angle Sample-Spinning NMR Spectroscopic Studies of Non-integral Spin Quadrupolar in Solids: Analysis of Spinning-Sidebands for the Case $\eta=0$. Chem Phys. Letl., 169:301, 1990.

[41] S. Ganapathy, S. S.hramm, and E. Oldlickl. Variable-Angle Spinning HighResolution NMIR of Solids. J. C'hem. Phys., 77:1360, 1982.

[42] J. H. Ballisberger. S. L. Ciamn, P. J. Cimandinctil, and A. Pines. CrossPolarization Dyoumic-:Angle Spinning Nuclear Magnetic Resonance of Quad-

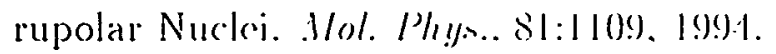

[43] T. P. Jarvic, R. M. Mrnslow, and K. 'l'. Mueller. Migh-Resolution Solid-State Heteronuclar ('orrelation S..MlR for Quadrupolar Nuclei. J. Am. C'hem. Soc., 117:570, 199:5.

[44] C. Dean, T. IV. Mambley, and M. R. Snow. Suructures of Phase IV Rubidium Nitrate, Rb:NO, and Plasc II ('osium Nibalc. ('sNO, Arla Cryst., 40:1512, 198.1.

[45] S. II. Wang. Z. Xu. Baltisberger .J. II., L. M. Bull, .J. F. Stebbins, and A. Pines. Multiple-Quantum Magic-Angle Spinning and Dynamic-Angle Spinning NMR Spectroscopy of Quadrupolar Ninclei. Solid Sirale NMR, S:1, 1997.

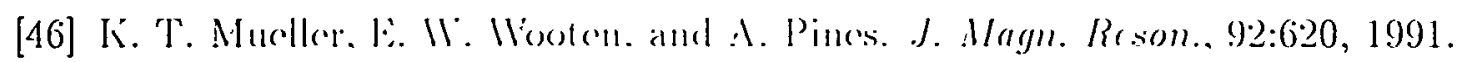

[47] P. J. Cimandinchli. J. II. Ballisherger I. Farman. J. F. Sucbbins, U. Werner, and

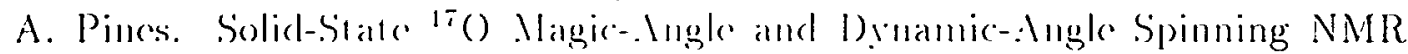

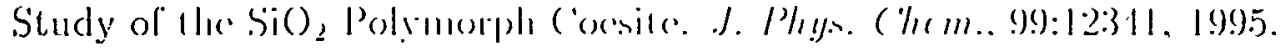

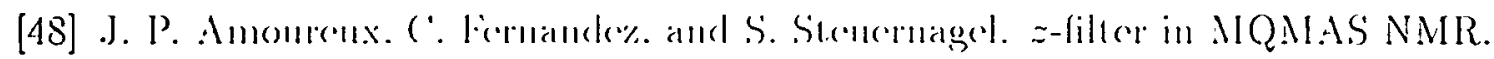
J. Mlagm. Rasom. Serins A. 12:116. I!g)(i.

[49] S. P. Brown. S. J. Ileyes. and S. IIImporis. 'TWo-Dimensional MAS MultipleQuantum NMlR of Quadrupolar Nurlei. Remoral of Inhomogencous Second-

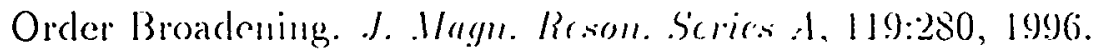


[50] P. J. Grandinet.li, .J. II. Ballisberger, A. Llor, Y. K. Lee, U. Werner, M. A. Eastman, and A. Pines. Pure-Absorption-Mode Lineshapes and Sensitivity in Two-Dimensional Dy'namic-Angle Spinning NMR.. J. Magn. Reson. Series A, 103:72, 1993.

[51] D. Massiot, B. Touzo, D. Trumeau, J. P. Coutures, J. Virlet, P. Florian, and P. J. Grandinetti. Two-Dimensional Magic-Angle Spinning Isotropic Reconstruction Sequences for Quadrupolar Nuclei. Solid State NMR, 6:73, 1996.

[52] K. Nagayama, P. Bachmann, K Wuthrich, and R. R. Ernst. J. Magn. Reson., $31: 133,1978$.

[53] A. Bax, R. Il. Griffey, and B. L. Hawkins. J. Mlagn. Reson., 55:301, 1983.

[54] A. C. Kolbert. M. II. [evill. and R. C. Griffin. J. Magn. Reson., 85:42, 1989.

[55] S. P. Brown and S. Wimpris. Two-Dimensional Multiple-Quantum MAS NMR of Quadrupolar Nuclei. Acquisition of Whole Echo. J. Magn. Reson., 124:279, 1997.

[56] P. J. Dirken, S. (. Kohn, M. E. Smith, and E. R. H. van Eck. Complete Resolution of Si-O-Si and Si-O-Al Plagments in an Aluminosilicate Glass by ${ }^{17} \mathrm{O}$ Multiple Quantum Magic Angle Spinning NMR Spectroscopy. Chem Phys. Lel.t., $266: 568,1997$.

[57] J. V. Hanna, M. E. Smith, and H. .J. Whitfield. Multiple Quantum Magic Angle Spinning NAIR detection of Impurity Phase in $\mathrm{Na}_{2} \mathrm{HrO}_{3}$. J. Am. Chem. Soc., 118:57\%2, 1996.

[5S] A. Medek. J. S Ilarwood. and L. Fydman. Hultiple-Quantum Magic-Angle Spinning NMIR: A Now Mothod lor the Study of Quadrupolar Nuclei in Solids. J. Am. (\%rm. Sor. 117:127T?, 1995.

[59] C. Jager, P. Ilantmann. (i. K. Fandroi. O. Hirsch, P. Rehak, J. Vogel, M. Feike, H. W. Spiess, K. Ilorog. and B. Thomas. Novel Oppertunities of Structural

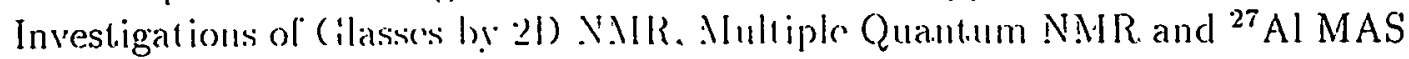
NMR Lineshape Analysis. Ber. Bumsongrs. Physs. Clhem., 100:1560, 1996.

[60] J. H. Baltisberger, Z. Xu, J. L. Stcbbins, S. H. Wang, and A. Pines. TripleQuantum 'Two-1)imensional ${ }^{2 T}$ Al Magic-Angle Spinning Nuclear Magnetic Resonance Spectroscopic Study of Aluminosilicalce and Aluminate Crystals and Glasses. J. Am. ('/16m. Soc., 118:7209), 1996. 
[61] J. Rocha, A. P. lisculcas, (:. Fomande\% and J. P. Amoureux. 'Two-Dimensional Triple-Quantum ${ }^{27}$ :I MAS NMIR Spectroscopic Study of the High-Temperature Phase Transformation of Microporous VPI-5. J. Phys. Chem., 100:17S89, 1996.

[62] S. H. Hwang, ( $:$ Fornande\%, J. P. Amoureux, J. Cho, S. W. Martin, and M. Pruski. Quantitative Study of the Short Range Order in $\mathrm{B}_{2} \mathrm{O}_{3}$ and $\mathrm{B}_{2} \mathrm{~S}_{3}$ by M $\Lambda S$ and Two-Dimonsional Triple-Quantum MAS" ${ }^{~ B ~ N M R . ~ S o l i d ~ S t a t e ~}$ NMR, 8:109, 19!).

[63] G. Wu, D. Rovnyak, and R. G. Cirillin. Quantitative Multiple-Quantum MagicAngle-Spinning NMR Spectroscopy of Quadrupolar Nucloi in Solids. J. Am. Chem. Soc.. 118:9:32(i. 19996.

[64] S. Vega. Ficlitious Sipiu 1/2 Operator lormalism for Multiple Quantum NMR. J. Chom. Phys., (is:is) 18 , 1978.

[65] A. Wokaun and R. R. Linst. Sodective Excitation and Detection in Multilevel Spin System: Applicalion of Single T'ransition Operators. J. Chem. Phys., $67: 1752,197 \pi$.

[66] E. R. Johuston. Densily Matrix 'Theory for ('alculating Magnetization Transfer and Dynamie Lineshape lifferts. ('oncrpts in M/cgn. Reson., 7:219, 1995.

[67] J. P. Amoureux, ('. Fornande\% and L. Frydman. Optimized Multiple-Quantum Magic-Angle Spinning NMIR Expreinconts on llalf-Integer Quadrupoles. Chem. Phys. Lell. 25!):347, 1!)!(i.

[68] G. Wu, D. Rovmaki. 13. Q. Sun. and R. (i. Cirillin. Iligh-Resolution Multiple Quantum M.AS N.IIR Spertroscope of IIalf-Intcger Quadrupolar Nuclei. Chem.

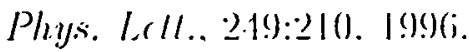

[69] A. J. Vega. MASS A.IIR Spin locking of Ilalf-Integer Quadrupolar Nuclei. J.

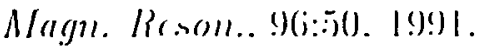

[70] A. J. Vega. MASs Spin-locking of Ilalf-Luteger (Quadrupolar Nuctei. J. Magn. Reson.. (96:50, 1!)!!.

[71] S. M. De Panl. M. Linst. J. S. Shore. J. I. Stoblins, and A. Pines. CrossPolarization from Quadrupolar Nuclei to Silicon Using Low-Radio-Frequency Amplitudes during .Magic-:Angle Spinning. J. Phys:. Chem., 101:3240, 1997.

[72] M. A. Bastman, P. J. Cirandinctli. I. K. Loe. and A. Pincs. Double-Tuned

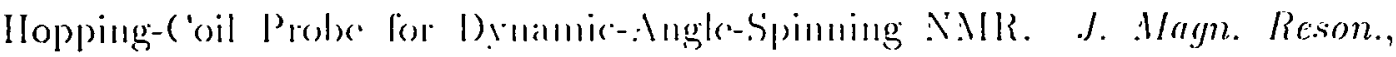


$98: 333,1992$.

[73] F. D. Doty and P. D. Ellis. Design of High Speed Cylindrical NMR Sample Spinners. Rev. Sci. Instr., 52:1868, 1981.

[74] D. Massiot, I. Farnan, N. Gautier, D. Trumeau, P. Florian, and P. J. Grandinelti. ${ }^{69} \mathrm{Ga}^{71}{ }^{7} \mathrm{C}$ ia Solid State Static, MAS and DAS NMR Study of $\beta$ $\mathrm{Ga}_{2} \mathrm{O}_{3}$. J. Chim. Phys.. 92:1847, 1995.

[75] K. T. Muellor, Y. Niu, B. F. C'hnelka, J. Stebbins, and A. Pines. HighResolution Oxygon-1i NiR of Solid Silicates. J. Am. Chem. Soc., 113:32, 1990.

[76] K. T. Mucller, (i. C. ('hingas, and A. Pines. NMR Probe for Dynamic-Angle Spinning. Rev. Scri. Ins/r.. 62:11415, 1991.

[77] M. Hanaya and R. K. Harris. Elfect of 'H-Decoupling in Tivo-Dimensional Multiple-Qnantum MAS NMR Sprectroscopy of ${ }^{23} \mathrm{Na}$ in a. Hydrous Layered Silicate. Solid stals NiM/R, S:1:17, 1997.

[78] J. F. Stebbins. Struclurr. Dymamics and Properties of Silicate Mells, vol. 32, page 191. Book Cirafters. Inc., C'hclsea, Michigan, 1995.

[79] C. Fernandez and J. P. Amourcix. 2D Multiquantum MAS-NMR Spectroscopy of Al-27 in Aluminaphosphate Nolecular Sieves. Chem. Phys. Letl., 242:449, 1995.

[80] I. Farnan, P. J. Cirandinctli. J. II. Ball isberger. J. F. Stebbins, U. Werner, M. A. Eastman, and A. Pincs. Quantification of the Disorder in Network-Modified Silicate Cilasses. Malure, 3is:31. 194:2.

[81] P. Florian, K. F. Vomillion. P. J. Cirandinct1. and J. P. Stobbins. Cation

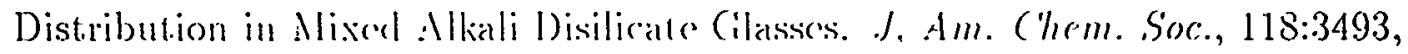
1996.

[S2] .J. H. Balt isberger. Adrences and Ipplicalions of Dynamic-Angle Spinning Nuclear Magnclic Rrsonancr. Phll) thesis. ('niversity' of California at. Berkeley, 1993.

[83] P. J. Grandinct1. Y. K. LC, J. 11. Ballisberger, B. Q. Sun, and A. Pines. Sideband Patucrus in Dyuamic-Angle Spiuning. J. Magn. Reson. Series A, 102:19:5, 19!):3. 
[84] J. S. Shore, S. II. Wang, R. F. 'Laylor, A. T. Bcll, and A. Pines. Determination of Quadrupolar and C.hemical Shiclding Tensors Using Solid-State TwoDimensional NMlR Spectroscopj. J. Chem. Phys., 105:9412, 1996.

[85] J. T. Cheng, J. ( : Edwards, and P. D. Ellis. Measurement of Quadrupolar Coupling Constants. Shickling Tensor Elements, and the Relative Orientation of Quadrupolar and Shiclding Tensor Principal Axis Systems for Rubidium-87 and Rubidium-\$.5 Nucloi in Rubidium Salt.s by Solid-State Nuclear Magnetic

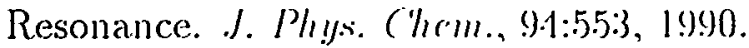

[86] A. Medek, J. R. Sachloben, P. Berempli. and I. Frydman. Multi-Rank NMR Studies of Ilalf-inloger Quadrupolar Nuclei in Solids by Three-Dimensional Dynamic-Angle Correlation Spectroscops:. J. Chcm. Phys., 104:5374, 1996.

[87] A. Samoson. E. I,ippmaa. and A. Pincs. High-Resolulion Solid State NMR, Averaging of Socond-()rede liflects by Mcans of a Jouble-Rolor. Mol. Phys., $65: 101: 3,19$ ถล่.

[88] A. Samoson and li. Lippmat. Synchronimed Double-Rotation NMR Spectroscopy. .J. M/rgm. Rr.son., 81:110. 1989.

[89] R. Jelinck, 13.l'. ('lunclka, Y'. Wu, M.li. Davis, J.G. Ulan, R. Gronsky, and A. Pines. Adsorplion Efferes in Aluminophosphate Molecular Sieves Studied by ${ }^{27} \mathrm{Al}$ Doublo-Rolation NMIR. ('alal. I.c1l., 15:(6.5, 1999.).

[90] R. Jolinck, 13.l'. ('mmellä. Y. Min. P..J. Cimninchi, A. Pines, P..J. Barrie, and J. Klinowski. Study of the Alumophosphaties $A l P()_{1}-21$ and $A l P()_{1}-25$ by ${ }^{27} \mathrm{Al}$

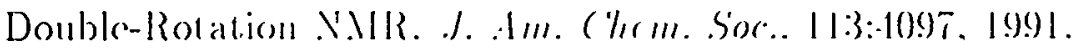

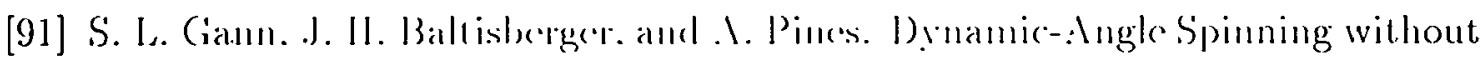

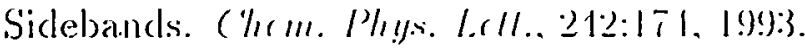

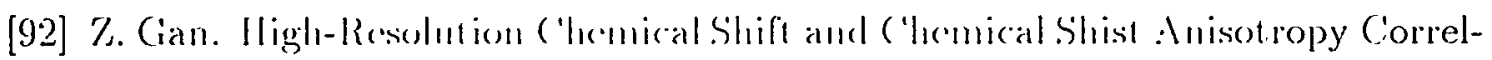

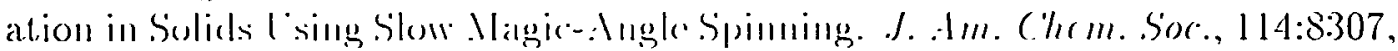
1992.

[93] .J. P. Amonrenx. ('. Fomande\% and F. Lafoldere. Sirlo-land Analysis in Variable-Anglo Samplo-Spimming (ViSSS) NMlR for 1.he ('ontral Transition of

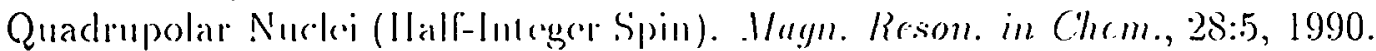

[94] J. M. Thomas and .). Klimowski. Adeamers in ('alalysis, vol. 3:3, page 199. Acadromic press. Inc.. lssis. 
[05] S. Schramm, R.. J. Kirkpat.rick, and E. Oldficld. Observation of High-Resolution Oxygen-17 NMR Spectra of Inorganic Solids(I). J. Am. Chem. Soc., 105:2483, 1983.

[96] H. C. Timken, C. L. Tumer, J. P. Gilson. L. B. Welsh, and E. Oldfield. SolidState Oxygen-17 Nuclear Magnelic Resonance Spectroscopic Studies of Zeolites and Related Systems(II). J. Am. Chem. Soc., 108:7231, 1986.

[97] H. C. Timken, N. Jancs, Ci. L. Turner, S. L. Lambert, L. B. Welsh, and E. Oldfield. Solid-State Oxygen-17 Nuclear Magnetic Resonance Spectroscopic Studjes of Zeolites and Rolated Systems. J. Am. Chem. Soc., 108:7236, 1986.

[98] S. Schramm and E. Oldficld. High-Resolution Oxygen-17 NMR. of Solids. J. Am. Chem. Soc., 106:2:502, 198.1.

[99] L. B. Alcmany, D. Massiol. B. L. Slicriff, M. E. Smith, and F. Taulelle. Observation and Accurale Quantificalion of ${ }^{2 \pi} \mathrm{Al}$ MAS NMR. Spectra of Some $\mathrm{Al}_{2} \mathrm{SiO}_{5}$ Polymorphs Conlaining Siles with Large Quadrupole Interactions. Chem Phys. Let..., $177: 301,1991$.

[100] E. Oldfield and R.J. Kirkpat rick. Iligh-Resolution Nuclear Magnetic Resonance of Inorganic Solids. S'cirncs, 227:1537, 1985.

[101] E. Oldfield, II. Ky'ung, ('. Timken, B. Montez, and R. Ramachandran. HighResolution Solid-Siate N'.tIR of Quadrupolar N'uclei. Naturc, 318:163, 1985.

[102] R. J. Kirkpatrick. R. Oestrike. ('. A. Hoiss. K. A. Sinith, and E. Okdlield. HighResolution ${ }^{27} \mathrm{Ml}$ and ${ }^{29} \mathrm{Si}$ NMIR Spectroscopy of Cilasses and ('rystals along the

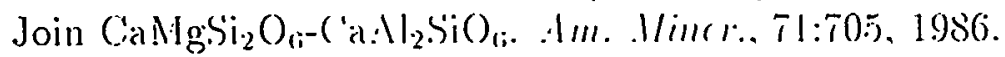

[103] C. I. Merzbacher. 13. L. Sherriff. J. S. Harman, and W: B. White. A HighResolution ${ }^{29} \mathrm{Si}$ and ${ }^{27} \mathrm{Al}$.MIR study of Alkaline Rarth Numinosilicate. J. of Non-Crysl. Solids. 12.1:19.1. 1990.

[104] R. Oestrike. IV. II. lang. R. J. Kirkpatrick. R. L. Hervig, A. Navrotsky, and

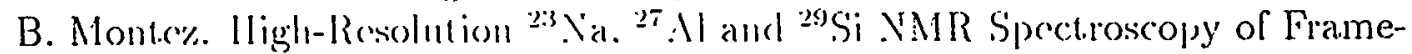
work Aluminosilicanc Cilassess. (iro('him. Cosmochimı. Acta. 51:2199), 1987.

[105] G. Engelhardt. N. Nol\%. K. Fockcl. F. Ci. Wilsmann, M Magi, A. Samosen, and E. Lippmaa. Structural Situdiess of Calcium Aluminosilicate Glasses by HighResolulion Solid Statc ${ }^{29}$ Si and ${ }^{2 \tau}$ : 11 Magic Angle Spinning Nuclear Magnetic

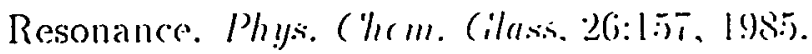


[106] S. F. I)ec, J. J. Fitrgerald. J. S. Prye. M. P. Shatlock, and G. E. Maciel. Observation of Six-coordinate Aluminum in and Alusite by Solid-State ${ }^{27} \mathrm{Al}$ MAS NAR. J. M/agn. R(son., (9:3:-10:3, 199!!.

[107] B. C. Bunker, R. J. Kirkpatrick, R. K. Brow, G. L. Turner, and C. Nelson. Local Structure of Alkalinc-liarth Boroahmminate Crystals and Cilasses: II. "B and ${ }^{27} \mathrm{Al}$ MAS N.MIR Sperelroscopy of Allialine-lisarth Boroaluminate Glasses. J. Am. Ceram. S'ore. 7-1:1.130. 1991.

[108] B. T. Poe. P. P. Mc.Mlillan, ('. A. Angell, and R. K. Sato. Al and Si Coordination in $\mathrm{SiO}_{2}-\mathrm{Al}_{2} \mathrm{O}_{3}$ Cilasses and licpuids: 1 Study by NMIR and IR Spectroscopy and MD Simulations. ('/n m. (irol.. 96:3:33, 19992.

[109] S. II. Risbud, R. J. Kirlipalick. A. P. Taglialavore, and B. Montez. SolidState NMIR Evidence of 1-. 5-, and (i-fold Alumimum Sites in Roller-Quenched $\mathrm{SiO}_{2}-\mathrm{Al}_{2} \mathrm{O}_{3}$ (ilasses. J. Alm. ('ram. Soc., 70:10. 1987.

[110] R. K. Salo. P. F. McMillan. P. I)omison. and R. Dupree. High-Resolution ${ }^{27} \mathrm{Al}$ and ${ }^{29} \mathrm{Si} \mathrm{M} A \mathrm{~S} \mathrm{NM}$ M lurestigation of $\mathrm{SiO}_{2}-\mathrm{Al}_{2} \mathrm{O}_{3}$ (ilasses. J. Phys. Chem., $95: 4.481,1991$.

[111] R. K. Sato, P. F. Mc.Millan, P. I) (rmison, and R. Duprece. A Structural Investigallion of High-. Mlumina ('ontenl (ilasses in $(\mathrm{aO})-\mathrm{Al}_{2} \mathrm{O}_{3}-\mathrm{SiO}_{2}$ System via

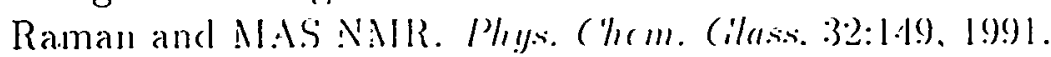

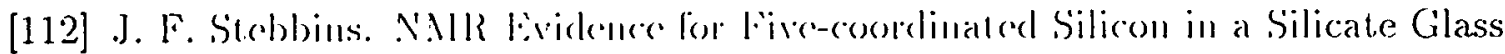

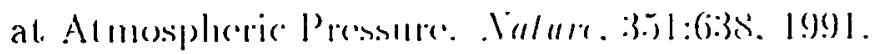

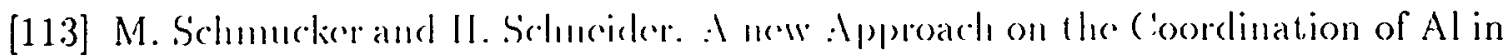

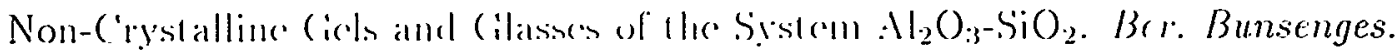

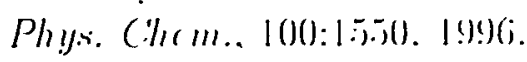

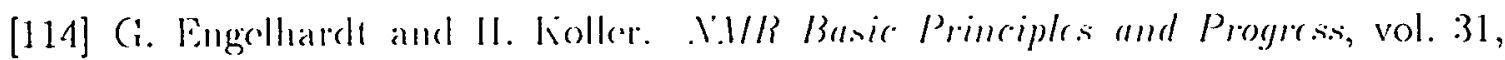
page 1. Springer-lorlag. Borlin Hoidcllorg. 19)1.

[115] J. Noucfoind and K. 1). Liss. Bond Angle l)ist rilution in Amorphous Germania

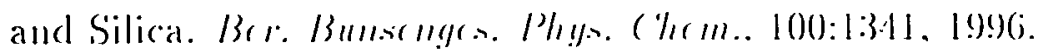

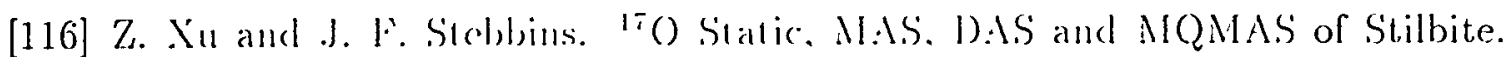
Solid Slale .VillR. in press.

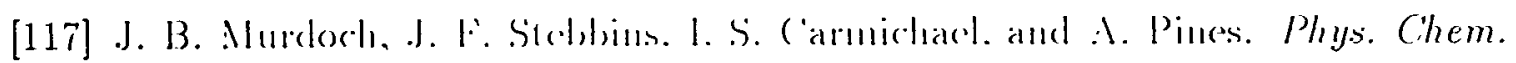
Mincr. 15:370. 194ה'. 
[118] B. L. Philips, R. J. Kirkpatrick, and M. A. Carpenter. Am. Miner., 77:484, 1992.

[119] S. C. Kohn, C. M. Henderson, and R. Dupree. Am. Miner., 80:705, 1995.

[120] I. W. Brown, C. M. Carclile, K. J. Mackenzie, M. J. Ryan, and R. H. Meinhold. Phys. Chem. Mincr., 15:78, 1987.

[121] B. L. Philips, R. J. Kirkpatrick, and A. Pulnis. Phys. Chem. Miner., 16:591, 1989.

[122] B. L. Phillips, R. J. Kirkpatrick, and A. Putnis. Phys. Chem. Miner., 16:591, 1989.

[123] S. Yang, K. D. Parka. and E. Oldlield. Oxygen-17 Labeling of Oxides and Zeolites. J. Am. Chrm. Sor.. 111:727s. 1989.

[124] T. J. Bastow and S. II. Stuar. " ${ }^{17}$ O NMR in Simple Oxides. Chem. Phys., $143: 459$, 1990.

[125] S. B. Alder, J. A. Raincr, J. Balt isberger, and U. Werner. Chemical Structure and Oxygen Dymanics in $13 \mathrm{a}_{2} \ln _{2} \mathrm{O}_{5}$. J. Am. Chem. Soc., 116:675, 1994.

[126] Z. Xu, J. V. Oglesby, and J. F. Stchbins. Disorder among Network Modifier Cations in Silicatco (ilasses: Vew Const mints from Triple-Quantum Oxygen-17 NMR. Am. llimer., in press.

[127] Z. Xu and J. F. Stobbins. "'O Static, MAS, DAS and MQNAS of Stibite. Solid Stalc NMIR, in press.

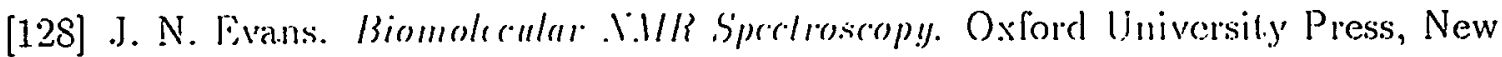
York. 190.5.

[129] M. Feike, R. (iraf. I. Solundl. ('. Jager. and H. W. Spiess. Structure of Crystalline Phosplates from P'-31 Doulsk-Q(nanl.um NMR Spectroscopy. J. Am. Chem. Soc.. 118:0)(3:31. 19996.

[130] D. Michol and F. lingdlke. N.M/R Basic Principles and Progress, vol. 32, page 69. Springer-Vorlag, Borlin Ileidelberg, 19993.

[131] S. L. Ciann, J. H. Baltisherger, li. W'. Wooten, H. Zimmermann, and A .Pines. Cross-Polarization and Denamic-Angle Spinning of ${ }^{17} \mathrm{O}$ in L-Alanine. Bull. of Magm. Rr.son., 1(6:6is. 19)!). 
[132] J. C. Edwards and P. 1). Villis. ('rosi-Polarization for Quadrupolar NucleiProton to Molybelcomm-95. Magn. Rcsom. in (Kem., 28:59, 1990.

[133] R. J. Kirkpatrick and R. K. Brow. Nuclear Magnetic Resonance Investigation of Structures of Phosphatr and Phosphatc-('ontaining Glasses: A Review. Solid Stale NMIR, 5:9. 19995.

[134] J. F. Baugher, P'. ('. 'Taylor. 'T. Oja, and P. J. Bray. Nuclcar Magnetic Resonance Powder Pattcrus in 1he Presence of ('ompletely Asymmetric Quadrupole and Chemical Shift Elleces: Application to Motavanadates. J. Chem. Phys., $50: 4911,1969$.

[135] Jr. Jones, W.Il. 'P'P. (iralan, and R. (i. Barmes. Nuclear Magnetic Resonance Lineshajess Resultiug from lle Combined Pffoces of Nuclear Quadrupole and

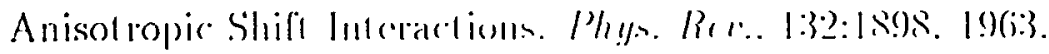

[136] M. Linder, A. Holener, and R. R. Frnst. Oricutation of Tensorial Interactions

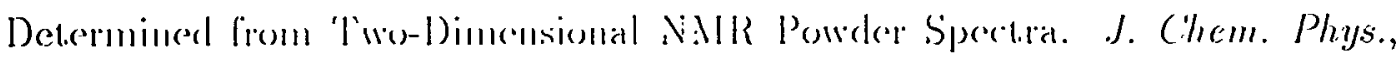

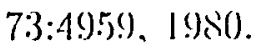

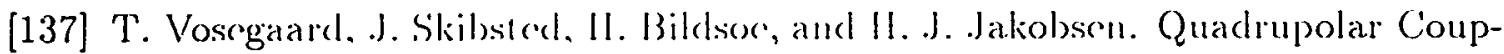
ling and Anisotropic Shickling from Single-('rystal NMR of the Cientral Trans-

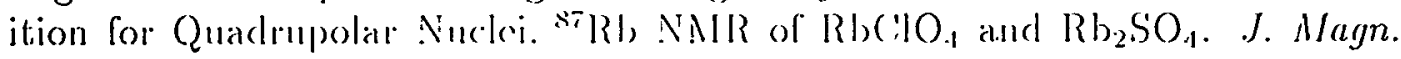
Reson., 12:2:111, 1!)!(j.

[138] P. Bodart, ('. Fomande\% and .J. P. Amonrenx. Rofincmont of Solid-State MAS NMR Spectra of Quadrupular Aurlei-Application to the Analysis of some V-51

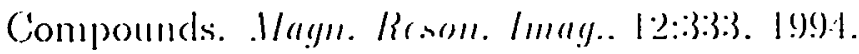

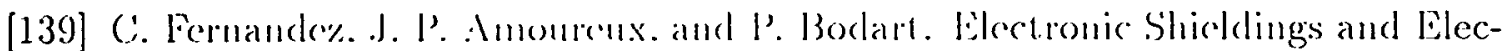
tric Ficld Ciradiculs in Rubidium Sulfate Sundied by Rb-Si MAS NMR. J. Magne. Rrson. Series A. 11:3:20:i. 1!)!5.

[140] J. Skibsted. X. ('. Nidsen. II. Biklsox. and II. J. Jakobsen. 51 V MAS NMIR Spectrosecopy: I)ereminalion of (Quadrupolar and Anisolropic Shielding 'Tousors. Indurling the Rolative () rientalion of 'Their Principal-Axis Systems.

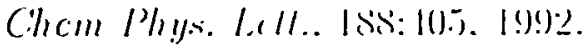

[141] J. Skibsted. N. ('. Nichorn. H. Milklsor. and II. J. Jakobsen. Magnitude and Relative Oriontalion of "sl' (Quadrupole (oupling and Ansotropic Shielding

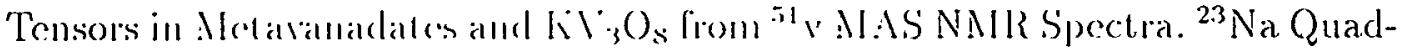

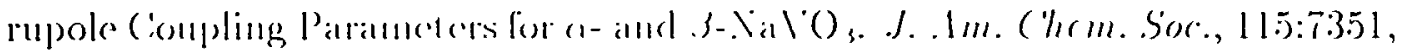
1993 . 
[142] A. Bax, N. M. Szeverenyi, and C. E. Maciel. Chemical Shift Anisotropy in Powdered Solids Studicd by 2D FT NMR with Flipping of the Spinning. $J$. Magn. Reson., 55:494, 1983.

[143] T. Terao, T. Fujii, T. Onodera, and A. Saikia. Chem. Phys. Lel.t., 107:145, 1984.

[144] L. Frydman, G. C. (') hingas, Y. K. I,ee, P. J. Girandinetti, M. A. Eastman, G. A. Barrall, and A. Pincs. Variable-Angle Correlation Spectroscopy in Solid-State Nuclear Magnetic Resonance. J. Chem. Plyys., 97:1800, 1992.

[145] S. Vega and A. Pines. Operator Formalism for Double Quantum NMR. J. Chem. Pliys. 66:56:21. 1977.

[146] S. Vega and Y. Nator. Triple-Quantum NMR. on Spin Systems with $I=3 / 2$ in Solicls. J. Chem. Physs., 7i):75, 1981.

[147] S. Vega, T. W. Shlatlutuck, and A. Pinces. Doublc-Quantum Cross-Polarization NMR in Solids. Phys.. Rro. A, 2:2:6:38, 1980.

[148] D. Massiot. Sensilivily and Lincshape Improvements of MQMAS by RotorSynchronized Dalia Acquisition. J. Magn. Reson. Series A, 122:240, 1996.

[149] C. Fernande\% and J. P. Amoureux. Triple-Quantum MAS-NMR of Quadrupolar Nuclei. Solid Stals Nil/R. 5:315. 19966.

[150] P. Sarv, C. Fornando, J. P. Amoureux, and K. Keskinen. Distribution of Tetrahedral Alumimm Sit.s in \%SM-5) Type Zcolites - an Al-27 (Multiquantum) Magic Angle Spinuing NaIR Study. J. Phys. (.hem., 100:1922.3, 1996.

[151] S. H. Wang, S. M. I) P'aul, and I. M. Bull. Iligh-Resolution Heteronuclear Correlation Bolween Quadrupolal and Spin-1/2 Nuclei Using Multiple-Quantum Magic-Angle Spinning. .J. Margn. Rrson. Sirrics A, 125:364, 1997.

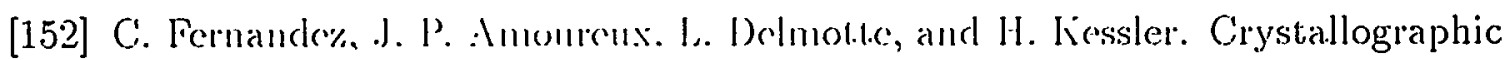
Site Probing in the 'lriclinic (ha-like AIPO) by Al-27 2D Quintuple-Quantum NMR. Hircoporous Malr rials. (6:12.5 19996.

[153] J. Rocha, \%. Liı, (․ Fernande\%, and J. P. Amoureux. Multiple-Quantum Al27 MAS NMR Spectroscopy of Microporous Aluminum Methlphosphonate $\beta$ AlMePO. C $\mathrm{hcm}$. Commum., 100:251:3, 1996.

[154] R. von Ballmoos and II: M. Ideler. Oxygen-18 Exchange between Zeolite ZSM-

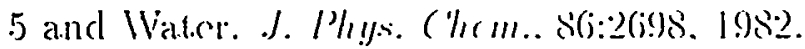

